\title{
Unsaturated Phosphonates as Hauser Acceptors for the Synthesis of Phosphonylated Dihydroxynaphthalenes and Naphthoquinones
}

Atul Kumar Chaturvedi, ${ }^{a}$ Namrata Rastogi*,a

${ }^{a}$ Medicinal \& Process Chemistry Division, CSIR-Central Drug Research Institute, B.S. 10/1, Sector 10, Jankipuram extension, Sitapur Road, Lucknow 226031, India

namrataiit@gmail.com; namrata.rastogi@cdri.res.in

\section{Supporting Information}

Table of Contents

\begin{tabular}{|c|c|c|}
\hline Entry & Description & $\begin{array}{c}\text { Page } \\
\text { No. }\end{array}$ \\
\hline 1. & Figure 1: ${ }^{1} \mathrm{H}$ NMR spectrum of $\mathbf{1 b}$ & 5. \\
\hline 2. & Figure 2: ${ }^{13} \mathrm{C}$ NMR spectrum of $\mathbf{1 b}$ & 5. \\
\hline 3. & Figure $3:{ }^{1} \mathrm{H}$ NMR spectrum of 1c & 6. \\
\hline 4. & Figure $4:{ }^{13} \mathrm{C}$ NMR spectrum of $1 \mathrm{c}$ & 6. \\
\hline 5. & Figure 5: ${ }^{1} \mathrm{H}$ NMR spectrum of $\mathbf{1 d}$ & 7. \\
\hline 6. & Figure 6: ${ }^{13} \mathrm{C}$ NMR spectrum of $\mathbf{1 d}$ & 7. \\
\hline 7. & Figure 7: ${ }^{1} \mathrm{H}$ NMR spectrum of $\mathbf{2 d}$ & 8. \\
\hline 8. & Figure 8: ${ }^{13} \mathrm{C}$ NMR spectrum of $\mathbf{2 d}$ & 8. \\
\hline 9. & Figure 9: ${ }^{31} \mathrm{P}$ NMR spectrum of $\mathbf{2 d}$ & 9. \\
\hline 10. & Figure 10: ${ }^{1} \mathrm{H}$ NMR spectrum of $\mathbf{2 e}$ & 9. \\
\hline 11. & Figure 11: ${ }^{13} \mathrm{C}$ NMR spectrum of $\mathbf{2 e}$ & 10. \\
\hline 12. & Figure 12: ${ }^{31} \mathrm{P}$ NMR spectrum of $\mathbf{2 e}$ & 10. \\
\hline 13. & Figure 13: ${ }^{1} \mathrm{H}$ NMR spectrum of $\mathbf{2 f}$ & 11. \\
\hline 14. & Figure 14: ${ }^{13} \mathrm{C}$ NMR spectrum of $\mathbf{2 f}$ & 11. \\
\hline 15. & Figure $15:{ }^{31} \mathrm{P}$ NMR spectrum of $\mathbf{2 f}$ & 12. \\
\hline 16. & Figure 16: ${ }^{1} \mathrm{H}$ NMR spectrum of $\mathbf{2} \mathbf{j}$ & 12. \\
\hline 17. & Figure 17: ${ }^{13} \mathrm{C}$ NMR spectrum of $\mathbf{2} \mathbf{j}$ & 13. \\
\hline 18. & Figure $18:{ }^{31} \mathrm{P}$ NMR spectrum of $\mathbf{2} \mathbf{j}$ & 13. \\
\hline 19. & Figure 19: ${ }^{1} \mathrm{H}$ NMR spectrum of $\mathbf{4 c}$ & 14. \\
\hline 20. & Figure 20: ${ }^{13} \mathrm{C}$ NMR spectrum of $\mathbf{4 c}$ & 14. \\
\hline 21. & Figure 21: ${ }^{31} \mathrm{P}$ NMR spectrum of $\mathbf{4 c}$ & 15. \\
\hline 22. & Figure 22: ${ }^{1} \mathrm{H}$ NMR spectrum of $\mathbf{4 d}$ & 15. \\
\hline 23. & Figure 23: ${ }^{13} \mathrm{C}$ NMR spectrum of $\mathbf{4 d}$ & 16. \\
\hline 24. & Figure $24:{ }^{31} \mathrm{P}$ NMR spectrum of $\mathbf{4 d}$ & 16. \\
\hline 25. & Figure 25: ${ }^{1} \mathrm{H}$ NMR spectrum of $\mathbf{4 e}$ & 17. \\
\hline 26. & Figure $26:{ }^{13} \mathrm{C}$ NMR spectrum of $4 \mathrm{e}$ & 17. \\
\hline 27. & Figure 27: ${ }^{31} \mathrm{P}$ NMR spectrum of $\mathbf{4 e}$ & 18. \\
\hline 28. & Figure $28:{ }^{1} \mathrm{H}$ NMR spectrum of $\mathbf{4 f}$ & 18. \\
\hline 29. & Figure 29: ${ }^{13} \mathrm{C}$ NMR spectrum of $\mathbf{4 f}$ & 19. \\
\hline 30. & Figure $30:{ }^{31} \mathrm{P}$ NMR spectrum of $\mathbf{4 f}$ & 19. \\
\hline 31. & Figure 31: ${ }^{1} \mathrm{H}$ NMR spectrum of $\mathbf{4 g}$ & 20. \\
\hline 32. & Figure $32:{ }^{13} \mathrm{C}$ NMR spectrum of $\mathbf{4 g}$ & 20. \\
\hline
\end{tabular}




\begin{tabular}{|c|c|c|}
\hline 33. & Figure $33:{ }^{31} \mathrm{P}$ NMR spectrum of $\mathbf{4 g}$ & 21. \\
\hline 34. & Figure 34: ${ }^{1} \mathrm{H}$ NMR spectrum of $\mathbf{4 h}$ & 21. \\
\hline 35. & Figure $35:{ }^{13} \mathrm{C}$ NMR spectrum of $\mathbf{4 h}$ & 22. \\
\hline 36. & Figure $36:{ }^{31} \mathrm{P}$ NMR spectrum of $\mathbf{4 h}$ & 22. \\
\hline 37. & Figure $37:{ }^{1} \mathrm{H}$ NMR spectrum of $\mathbf{4 i}$ & 23. \\
\hline 38. & Figure $38:{ }^{13} \mathrm{C}$ NMR spectrum of $\mathbf{4 i}$ & 23. \\
\hline 39. & Figure $39:{ }^{31} \mathrm{P}$ NMR spectrum of $\mathbf{4 i}$ & 24. \\
\hline 40. & Figure $40:{ }^{1} \mathrm{H}$ NMR spectrum of $\mathbf{4} \mathbf{j}$ & 24. \\
\hline 41. & Figure $41:{ }^{13} \mathrm{C}$ NMR spectrum of $\mathbf{4} \mathbf{j}$ & 25. \\
\hline 42. & Figure $42:{ }^{31} \mathrm{P}$ NMR spectrum of $\mathbf{4 j}$ & 25. \\
\hline 43. & Figure 43: ${ }^{1} \mathrm{H}$ NMR spectrum of $\mathbf{4 k}$ & 26. \\
\hline 44. & Figure $44:{ }^{13} \mathrm{C}$ NMR spectrum of $\mathbf{4 k}$ & 26. \\
\hline 45. & Figure $45:{ }^{31} \mathrm{P}$ NMR spectrum of $\mathbf{4 k}$ & 27. \\
\hline 46. & Figure 46: ${ }^{1} \mathrm{H}$ NMR spectrum of $\mathbf{3 a}$ & 27. \\
\hline 47. & Figure $47:{ }^{13} \mathrm{C}$ NMR spectrum of $\mathbf{3 a}$ & 28. \\
\hline 48. & Figure $48:{ }^{13} \mathrm{C}$ NMR spectrum of $\mathbf{3 a}$ (expansion) & 28. \\
\hline 49. & Figure $49:{ }^{31} \mathrm{P}$ NMR spectrum of $\mathbf{3 a}$ & 29. \\
\hline 50. & Figure 50: ${ }^{1} \mathrm{H}$ NMR spectrum of $\mathbf{3 b}$ & 29. \\
\hline 51. & Figure $51:{ }^{13} \mathrm{C}$ NMR spectrum of $\mathbf{3 b}$ & 30. \\
\hline 52. & Figure 52: ${ }^{13} \mathrm{C}$ NMR spectrum of $\mathbf{3 b}$ (expansion) & 30 . \\
\hline 53. & Figure 53: ${ }^{31} \mathrm{P}$ NMR spectrum of $\mathbf{3 b}$ & 31. \\
\hline 54. & Figure 54: ${ }^{1} \mathrm{H}$ NMR spectrum of $\mathbf{3 c}$ & 31. \\
\hline 55. & Figure 55: ${ }^{13} \mathrm{C}$ NMR spectrum of $\mathbf{3 c}$ & 32. \\
\hline 56. & Figure 56: ${ }^{13} \mathrm{C}$ NMR spectrum of 3c (expansion) & 32. \\
\hline 57. & Figure $57:{ }^{31} \mathrm{P}$ NMR spectrum of $\mathbf{3 c}$ & 33. \\
\hline 58. & Figure 58: ${ }^{1} \mathrm{H}$ NMR spectrum of $\mathbf{3 d}$ & 33. \\
\hline 59. & Figure 59: ${ }^{13} \mathrm{C}$ NMR spectrum of $\mathbf{3 d}$ & 34. \\
\hline 60. & Figure 60: ${ }^{13} \mathrm{C}$ NMR spectrum of $\mathbf{3 d}$ (expansion) & 34. \\
\hline 61. & Figure 61: ${ }^{31} \mathrm{P}$ NMR spectrum of $\mathbf{3 d}$ & 35. \\
\hline 62. & Figure 62: ${ }^{1} \mathrm{H}$ NMR spectrum of $\mathbf{3 e}$ & 35. \\
\hline 63. & Figure 63: ${ }^{13} \mathrm{C}$ NMR spectrum of $\mathbf{3 e}$ & 36. \\
\hline 64. & Figure $64:{ }^{13} \mathrm{C}$ NMR spectrum of $\mathbf{3 e}$ (expansion) & 36. \\
\hline 65. & Figure $65:{ }^{31} \mathrm{P}$ NMR spectrum of $3 \mathrm{e}$ & 37. \\
\hline 66. & Figure 66: ${ }^{1} \mathrm{H}$ NMR spectrum of $\mathbf{3 f}$ & 37. \\
\hline 67. & Figure 67: ${ }^{13} \mathrm{C}$ NMR spectrum of $\mathbf{3 f}$ & 38. \\
\hline 68. & Figure 68: ${ }^{13} \mathrm{C}$ NMR spectrum of $\mathbf{3 f}$ (expansion) & 38. \\
\hline 69. & Figure $69:{ }^{31} \mathrm{P}$ NMR spectrum of $\mathbf{3 f}$ & 39. \\
\hline 70. & Figure 70: ${ }^{1} \mathrm{H}$ NMR spectrum of $\mathbf{3 g}$ & 39. \\
\hline 71. & Figure 71: ${ }^{13} \mathrm{C}$ NMR spectrum of $\mathbf{3 g}$ & 40. \\
\hline 72. & Figure 72: ${ }^{13} \mathrm{C}$ NMR spectrum of $\mathbf{3 g}$ (expansion) & 40. \\
\hline 73. & Figure $73:{ }^{31} \mathrm{P}$ NMR spectrum of $\mathbf{3 g}$ & 41. \\
\hline 74. & Figure 74: ${ }^{1} \mathrm{H}$ NMR spectrum of $\mathbf{3 h}$ & 41. \\
\hline 75. & Figure $75:{ }^{13} \mathrm{C}$ NMR spectrum of $\mathbf{3 h}$ & 42. \\
\hline 76. & Figure 76: ${ }^{13} \mathrm{C}$ NMR spectrum of $\mathbf{3 h}$ (expansion) & 42. \\
\hline 77. & Figure $77:{ }^{31} \mathrm{P}$ NMR spectrum of $\mathbf{3 h}$ & 43. \\
\hline 78. & Figure 78: ${ }^{1} \mathrm{H}$ NMR spectrum of $\mathbf{3 i}$ & 43. \\
\hline 79. & Figure $79:{ }^{13} \mathrm{C}$ NMR spectrum of $\mathbf{3 i}$ & 44. \\
\hline 80. & Figure $80:{ }^{13} \mathrm{C}$ NMR spectrum of $\mathbf{3 i}$ (expansion) & 44. \\
\hline
\end{tabular}




\begin{tabular}{|c|c|c|}
\hline 81. & Figure $81:{ }^{31} \mathrm{P}$ NMR spectrum of $\mathbf{3 i}$ & 45. \\
\hline 82. & Figure 82: ${ }^{1} \mathrm{H}$ NMR spectrum of $\mathbf{3 j}$ & 45. \\
\hline 83. & Figure $83:{ }^{13} \mathrm{C}$ NMR spectrum of $\mathbf{3} \mathbf{j}$ & 46. \\
\hline 84. & Figure 84: ${ }^{13} \mathrm{C}$ NMR spectrum of $\mathbf{3 j}$ (expansion) & 46. \\
\hline 85. & Figure $85:{ }^{31} \mathrm{P}$ NMR spectrum of $\mathbf{3 j}$ & 47. \\
\hline 86. & Figure $86:{ }^{1} \mathrm{H}$ NMR spectrum of $\mathbf{3 k}$ & 47. \\
\hline 87. & Figure $87:{ }^{13} \mathrm{C}$ NMR spectrum of $\mathbf{3 k}$ & 48. \\
\hline 88. & Figure $88:{ }^{13} \mathrm{C}$ NMR spectrum of $\mathbf{3 k}$ (expansion) & 48. \\
\hline 89. & Figure $89:{ }^{31} \mathrm{P}$ NMR spectrum of $\mathbf{3 k}$ & 49. \\
\hline 90. & Figure 90: ${ }^{1} \mathrm{H}$ NMR spectrum of $\mathbf{3 l}$ & 49. \\
\hline 100. & Figure 91: ${ }^{13} \mathrm{C}$ NMR spectrum of $\mathbf{3 I}$ & 50. \\
\hline 101. & Figure 92: ${ }^{13} \mathrm{C}$ NMR spectrum of 3I (expansion) & 50. \\
\hline 102. & Figure $93:{ }^{31} \mathrm{P}$ NMR spectrum of $3 \mathrm{I}$ & 51. \\
\hline 103. & Figure 94: ${ }^{1} \mathrm{H}$ NMR spectrum of $\mathbf{3 m}$ & 51. \\
\hline 104. & Figure $95:{ }^{13} \mathrm{C}$ NMR spectrum of $\mathbf{3 m}$ & 52. \\
\hline 105. & Figure $96:{ }^{13} \mathrm{C}$ NMR spectrum of $\mathbf{3 m}$ (expansion) & 52. \\
\hline 106. & Figure $97:{ }^{31} \mathrm{P}$ NMR spectrum of $\mathbf{3 m}$ & 53. \\
\hline 107. & Figure 98: ${ }^{1} \mathrm{H}$ NMR spectrum of $\mathbf{3 n}$ & 53. \\
\hline 108. & Figure 99: ${ }^{13} \mathrm{C}$ NMR spectrum of $\mathbf{3 n}$ & 54. \\
\hline 109. & Figure 100: ${ }^{13} \mathrm{C}$ NMR spectrum of $\mathbf{3 n}$ (expansion) & 54. \\
\hline 110. & Figure $101:{ }^{31} \mathrm{P}$ NMR spectrum of $\mathbf{3 n}$ & 55. \\
\hline 111. & Figure 102: ${ }^{1} \mathrm{H}$ NMR spectrum of $\mathbf{3 o}$ & 55. \\
\hline 112. & Figure 103: ${ }^{13} \mathrm{C}$ NMR spectrum of $\mathbf{3 o}$ & 56. \\
\hline 113. & Figure 104: ${ }^{13} \mathrm{C}$ NMR spectrum of $\mathbf{3 o}$ (expansion) & 56. \\
\hline 114. & Figure $105:{ }^{31} \mathrm{P}$ NMR spectrum of $\mathbf{3 o}$ & 57. \\
\hline 115. & Figure 106: ${ }^{1} \mathrm{H}$ NMR spectrum of $\mathbf{3 p}$ & 57. \\
\hline 116. & Figure 107: ${ }^{13} \mathrm{C}$ NMR spectrum of $\mathbf{3 p}$ & 58. \\
\hline 117. & Figure 108: ${ }^{13} \mathrm{C}$ NMR spectrum of $\mathbf{3 p}$ (expansion) & 58. \\
\hline 118. & Figure 109: ${ }^{31} \mathrm{P}$ NMR spectrum of $\mathbf{3 p}$ & 59. \\
\hline 119. & Figure 110: ${ }^{1} \mathrm{H}$ NMR spectrum of $\mathbf{3 q}$ & 59. \\
\hline 120. & Figure 111: ${ }^{13} \mathrm{C}$ NMR spectrum of $\mathbf{3 q}$ & 60. \\
\hline 121. & Figure 112: ${ }^{13} \mathrm{C}$ NMR spectrum of $\mathbf{3 q}$ (expansion) & 60. \\
\hline 122. & Figure 113: ${ }^{31} \mathrm{P}$ NMR spectrum of $\mathbf{3 q}$ & 61. \\
\hline 123. & Figure 114: ${ }^{1} \mathrm{H}$ NMR spectrum of $\mathbf{5 a}$ & 61. \\
\hline 124. & Figure 115: ${ }^{13} \mathrm{C}$ NMR spectrum of $\mathbf{5 a}$ & 62. \\
\hline 125. & Figure 116: ${ }^{13} \mathrm{C}$ NMR spectrum of $\mathbf{5 a}$ (expansion) & 62. \\
\hline 126. & Figure $117:{ }^{31} \mathrm{P}$ NMR spectrum of $\mathbf{5 a}$ & 63. \\
\hline 127. & Figure 118: ${ }^{1} \mathrm{H}$ NMR spectrum of $\mathbf{5 b}$ & 63. \\
\hline 128. & Figure 119: ${ }^{13} \mathrm{C}$ NMR spectrum of $\mathbf{5 b}$ & 64. \\
\hline 129. & Figure 120: ${ }^{13} \mathrm{C}$ NMR spectrum of $\mathbf{5 b}$ (expansion) & 64. \\
\hline 130. & Figure $121:{ }^{31} \mathrm{P}$ NMR spectrum of $\mathbf{5 b}$ & 65. \\
\hline 131. & Figure 122: ${ }^{1} \mathrm{H}$ NMR spectrum of $\mathbf{5 c}$ & 65. \\
\hline 132. & Figure $123:{ }^{13} \mathrm{C}$ NMR spectrum of $\mathbf{5 c}$ & 66. \\
\hline 133. & Figure 124: ${ }^{13} \mathrm{C}$ NMR spectrum of $\mathbf{5 c}$ (expansion) & 66. \\
\hline 134. & Figure $125:{ }^{31} \mathrm{P}$ NMR spectrum of $\mathbf{5 c}$ & 67. \\
\hline 135. & Figure 126: ${ }^{1} \mathrm{H}$ NMR spectrum of $\mathbf{5 d}$ & 67. \\
\hline 136. & Figure 127: ${ }^{13} \mathrm{C}$ NMR spectrum of $\mathbf{5 d}$ & 68. \\
\hline 137. & Figure 128: ${ }^{13} \mathrm{C}$ NMR spectrum of $\mathbf{5 d}$ (expansion) & 68. \\
\hline
\end{tabular}




\begin{tabular}{|c|c|c|}
\hline 138. & Figure $129:{ }^{31} \mathrm{P}$ NMR spectrum of $\mathbf{5 d}$ & 69. \\
\hline 139. & Figure 130: ${ }^{1} \mathrm{H}$ NMR spectrum of $\mathbf{5 e}$ & 69. \\
\hline 140. & Figure 131: ${ }^{13} \mathrm{C}$ NMR spectrum of $\mathbf{5 e}$ & 70. \\
\hline 141. & Figure 132: ${ }^{13} \mathrm{C}$ NMR spectrum of $\mathbf{5 e}$ (expansion) & 70. \\
\hline 142. & Figure $133:{ }^{31} \mathrm{P}$ NMR spectrum of $\mathbf{5 e}$ & 71. \\
\hline 143. & Figure 134: ${ }^{1} \mathrm{H}$ NMR spectrum of $\mathbf{5 f}$ & 71. \\
\hline 144. & Figure 135: ${ }^{13} \mathrm{C}$ NMR spectrum of $\mathbf{5 f}$ & 72. \\
\hline 145. & Figure 136: ${ }^{13} \mathrm{C}$ NMR spectrum of $\mathbf{5 f}$ (expansion) & 72. \\
\hline 146. & Figure 137: ${ }^{13} \mathrm{C}$ NMR spectrum of $\mathbf{5 f}$ (expansion-2) & 73. \\
\hline 147. & Figure 138: ${ }^{31} \mathrm{P}$ NMR spectrum of $\mathbf{5 f}$ & 73. \\
\hline 148. & Figure 139: ${ }^{1} \mathrm{H}$ NMR spectrum of $\mathbf{5 g}$ & 74. \\
\hline 149. & Figure 140: ${ }^{13} \mathrm{C}$ NMR spectrum of $\mathbf{5 g}$ & 74. \\
\hline 150. & Figure 141: ${ }^{13} \mathrm{C}$ NMR spectrum of $\mathbf{5 g}$ (expansion) & 75. \\
\hline 151. & Figure $142:{ }^{31} \mathrm{P}$ NMR spectrum of $\mathbf{5 g}$ & 75. \\
\hline 152. & Figure 143: ${ }^{1} \mathrm{H}$ NMR spectrum of $\mathbf{5 h}$ & 76. \\
\hline 153. & Figure $144:{ }^{13} \mathrm{C}$ NMR spectrum of $\mathbf{5 h}$ & 76. \\
\hline 154. & Figure $145:{ }^{13} \mathrm{C}$ NMR spectrum of $\mathbf{5 h}$ (expansion) & 77. \\
\hline 155. & Figure 146: ${ }^{31} \mathrm{P}$ NMR spectrum of $\mathbf{5 h}$ & 77. \\
\hline
\end{tabular}




\section{NRAT-IV-125}

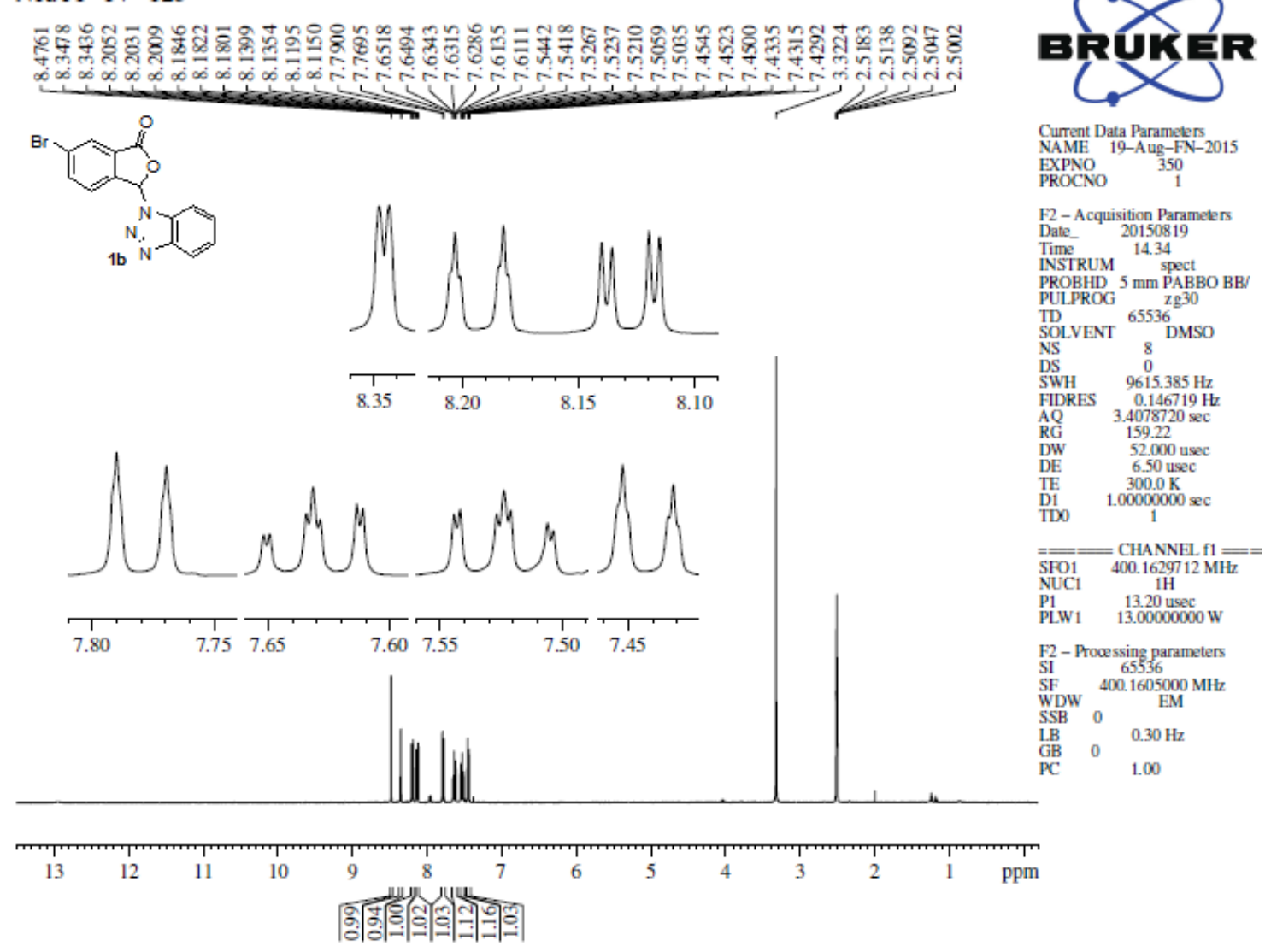

Figure 1: ${ }^{1} \mathrm{H}$ NMR spectrum of $1 \mathrm{~b}$

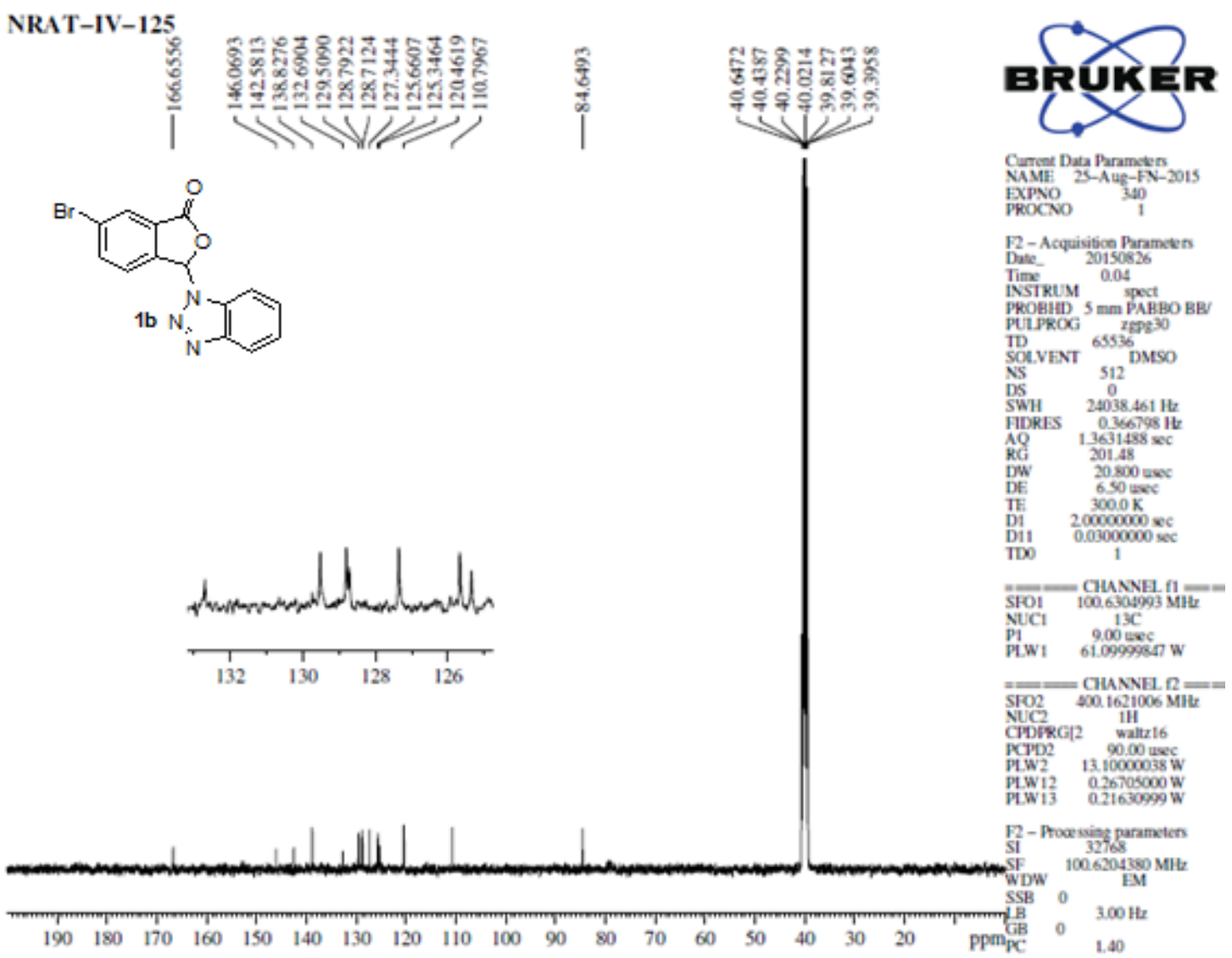

Figure $2:{ }^{13} \mathrm{C}$ NMR spectrum of $1 \mathrm{~b}$ 


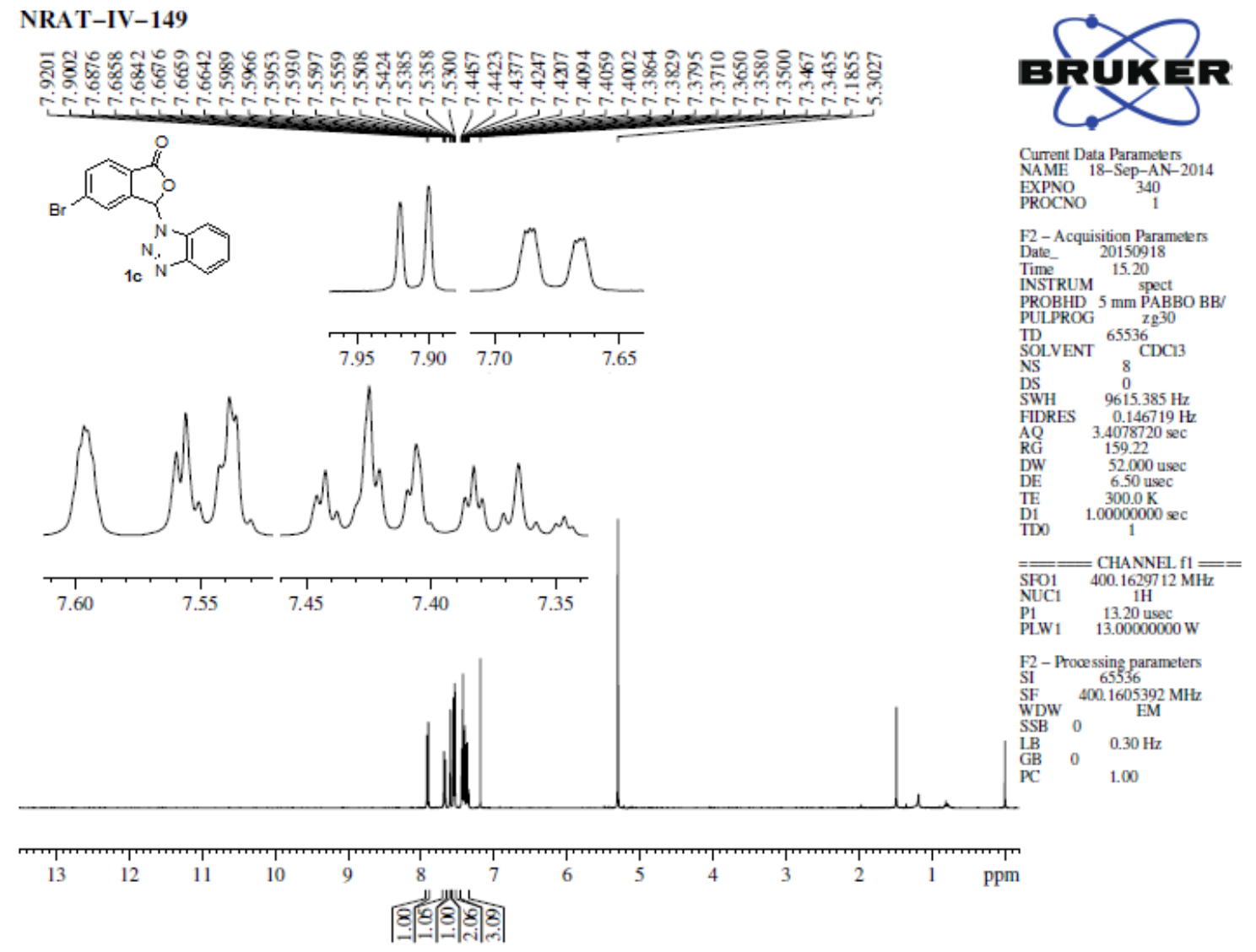

Figure 3: ${ }^{1} \mathrm{H}$ NMR spectrum of 1c
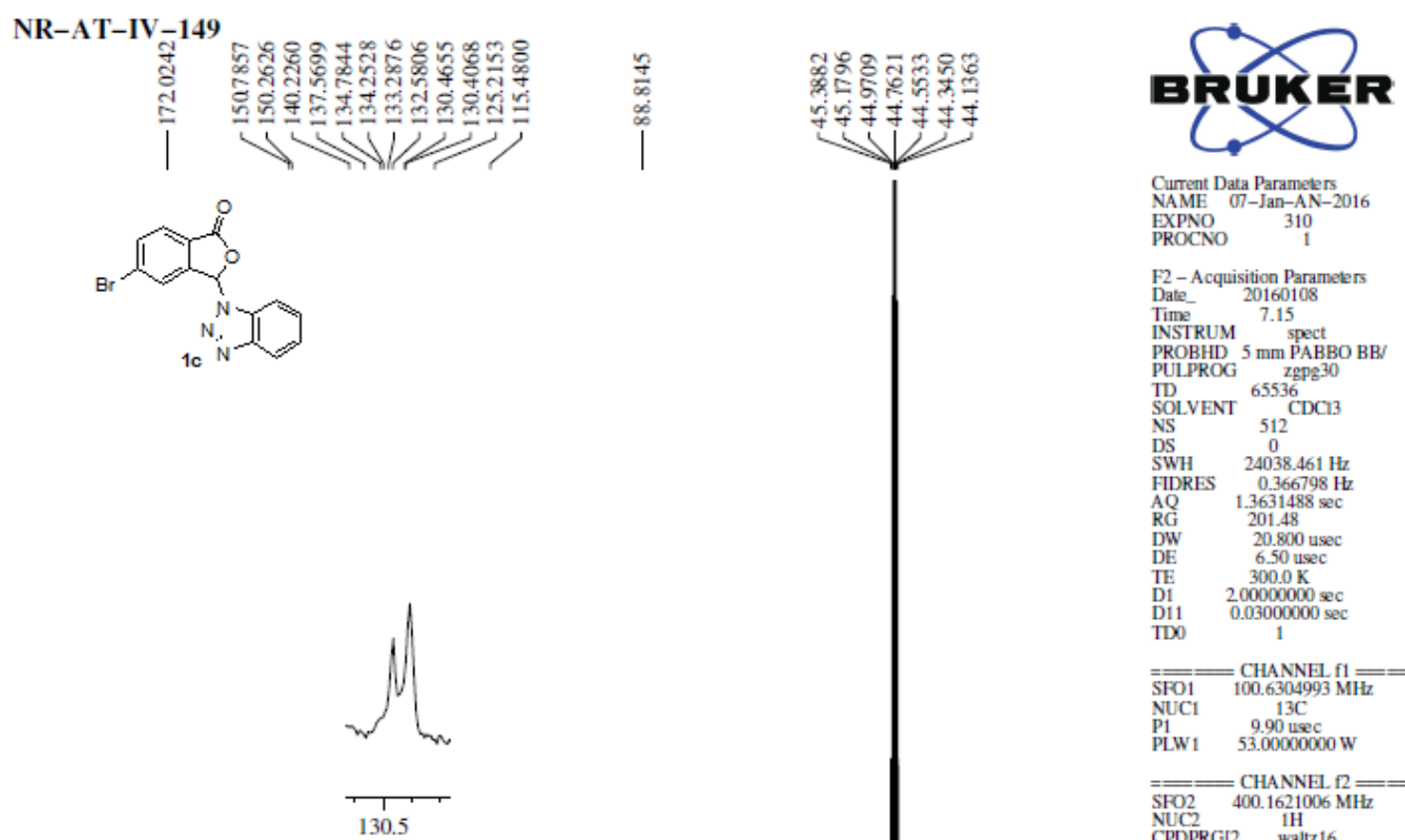

Current Data Parameters
NAME 07 -Jan-AN-2016 $\begin{array}{lr}\text { EXPNO } & 310 \\ \text { PROCNO } & 1\end{array}$

F2 - Acquisition Parameters

Time 7.15

NNSTRUM spect
PROBHD $5 \mathrm{~mm}$ PABBO BB

${ }_{\text {PULPROG }}{ }_{\text {ZgP:30 }}$

TD 65536

SOLVENT ${ }_{512} \mathrm{CDC}$

DS 0

SWH $\quad 24038.461 \mathrm{~Hz}$

FIDRES $\quad 0.366798 \mathrm{Ht}$

AQ $\quad 1.3631488 \mathrm{sec}$

DW $\quad 20.800$ usec 6.50 usec
$300.0 \mathrm{~K}$ $300.0 \mathrm{~K}$

$\begin{array}{ll}\text { D1 } & 200000000 \mathrm{sec} \\ \text { D11 } & 0.03000000 \mathrm{sec}\end{array}$

TDO $\quad 1$

$\overline{\mathrm{SFO} 1}=\underset{100.6304993 \mathrm{MHz}}{\text { CHANNE }} \mathrm{f1}=$

NUC1 $\quad 13 \mathrm{C}$

$\begin{array}{ll}\text { P1 } & 9.90 \mathrm{usec} \\ \text { PLW1 } & 53.00000000 \mathrm{~W}\end{array}$

$=\overline{=}$ CHANNEL $\mathrm{f} 2=$

$\mathrm{SFO} 2 \quad 400.1621006 \mathrm{M}$

CPDPRG12 $\quad \stackrel{1 \mathrm{H}}{\text { waltz16 }}$

PCPD2 90.00 usec

PLW12 $0.27963999 \mathrm{~W}$

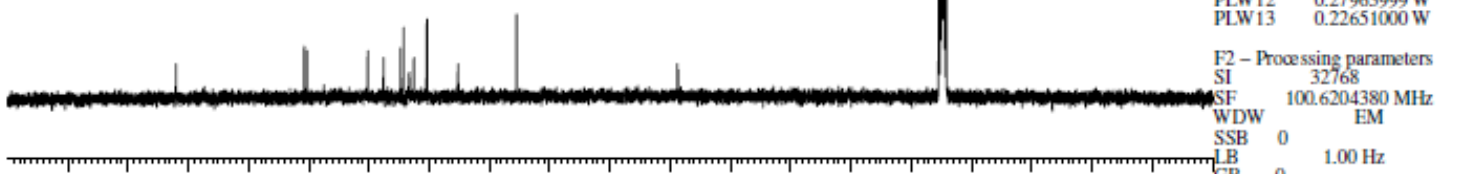

$\begin{array}{llllllllllllllllllll}190 & 180 & 170 & 160 & 150 & 140 & 130 & 120 & 110 & 100 & 90 & 80 & 70 & 60 & 50 & 40 & 30 & 20 & \text { Ppm }\end{array}$

Figure 4: ${ }^{13} \mathrm{C}$ NMR spectrum of $1 \mathrm{c}$ 


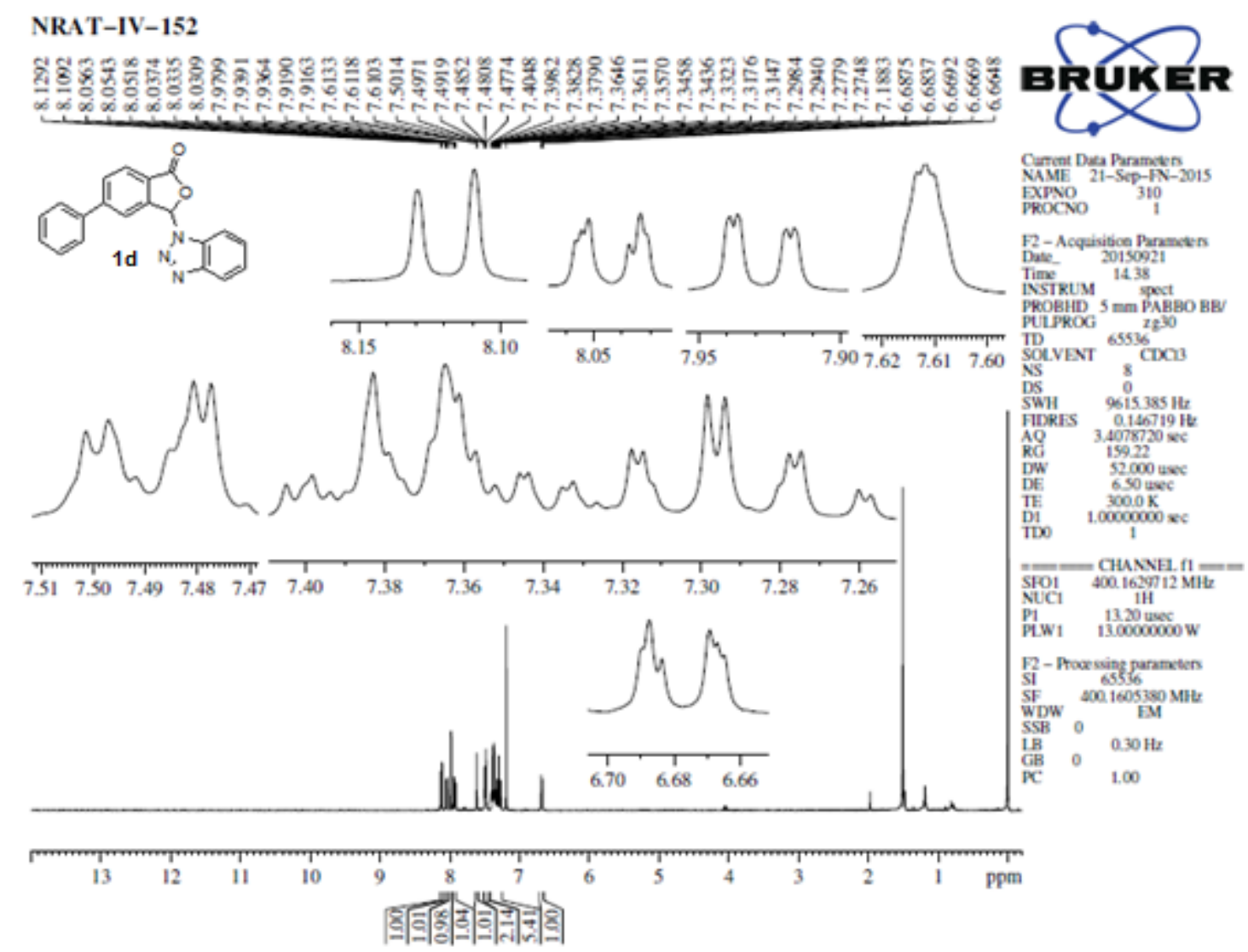

Figure 5: ${ }^{1} \mathrm{H}$ NMR spectrum of $1 \mathrm{~d}$

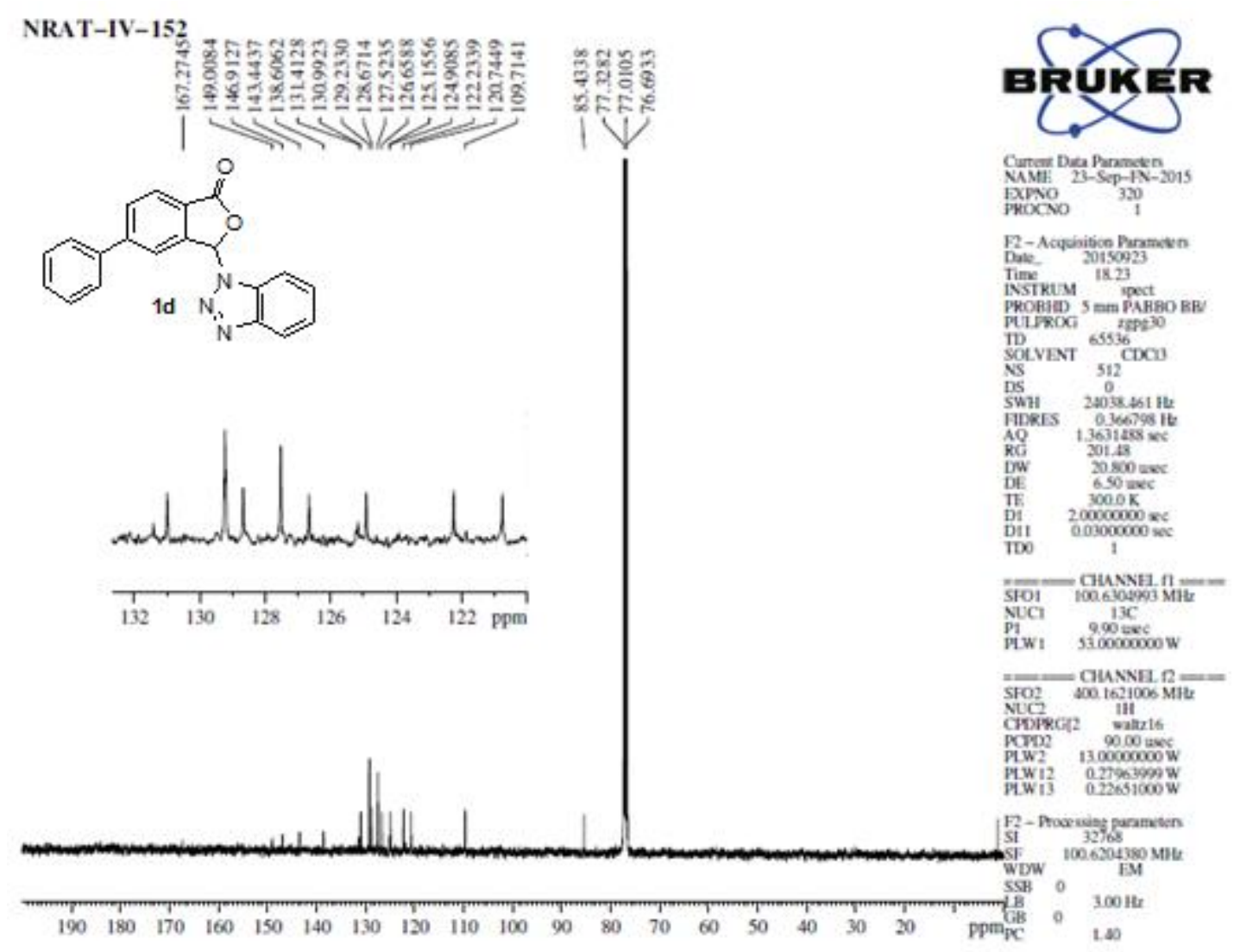

Figure 6: ${ }^{13} \mathrm{C}$ NMR spectrum of $1 \mathrm{~d}$ 
<smiles>COP(=O)(C=Cc1ccc(Br)cc1)OC</smiles>

Current Data Paramelers NAME 28-Dec-FN-2015 EXPNO
PROCNO

F2 - Acquisition Parameters

$\begin{array}{ll}\text { Date__ } & 20151228 \\ \text { Time } 17.18\end{array}$

INSTRUM spect PROBHD $5 \mathrm{~mm}$ PABBO B

TD 65536 (200

$1 /$
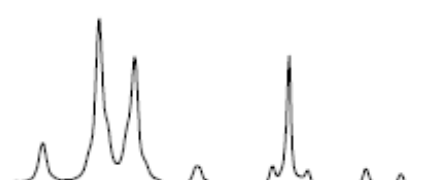

$\begin{array}{llllllll}7.45 & 7.40 & 7.35 & 7.30 & 7.25 & 7.20 & 7.15 & 7.10\end{array}$

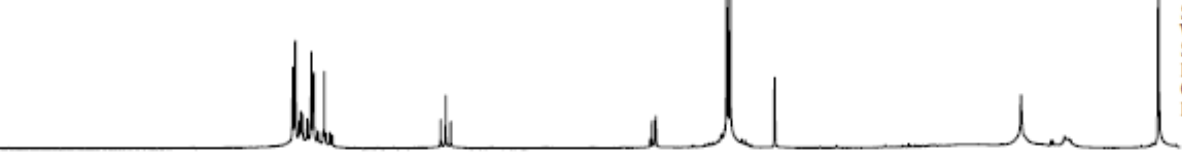

NS

$\begin{array}{ll}8 \\ \text { S } & 0\end{array}$

$9615.385 \mathrm{~Hz}$

FIDRES $0.146719 \mathrm{H}$

RG $\quad \begin{array}{ll}414.26 \\ \text { RG }\end{array}$

$\begin{array}{ll}\text { DW } & 52.000 \text { usec } \\ \text { DE } & 6.50 \text { usec }\end{array}$

TE $295.1 \mathrm{~K}$

DD1 $1.00000000 \mathrm{sec}$

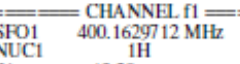

$\begin{array}{ll}\text { P1 } & 13.20 \text { usec } \\ \text { PLW1 } & 13.00000000 \mathrm{~W}\end{array}$

F2 - Processing parameters

SF $\quad 400.1605391 \mathrm{MHz}$

NSB 0 EM

LB $0.30 \mathrm{H}$

$\begin{array}{lll}\mathrm{GB} & 0 & \\ \mathrm{PC} & & 1.00\end{array}$

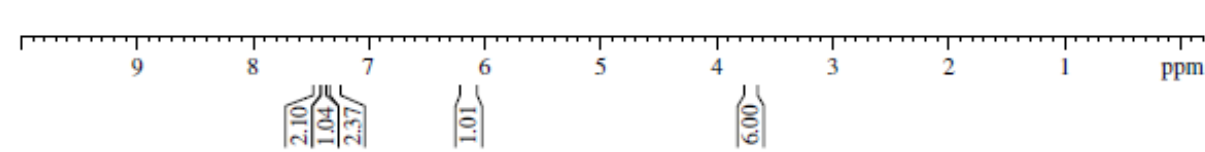

Figure 7: ${ }^{1} \mathrm{H}$ NMR spectrum of $2 \mathrm{~d}$

NR-AT-IV-196

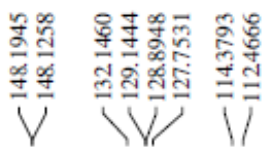

굴요

है

$\overbrace{2 \mathrm{~d}}^{\mathrm{PO}(\mathrm{OMe})_{2}}$
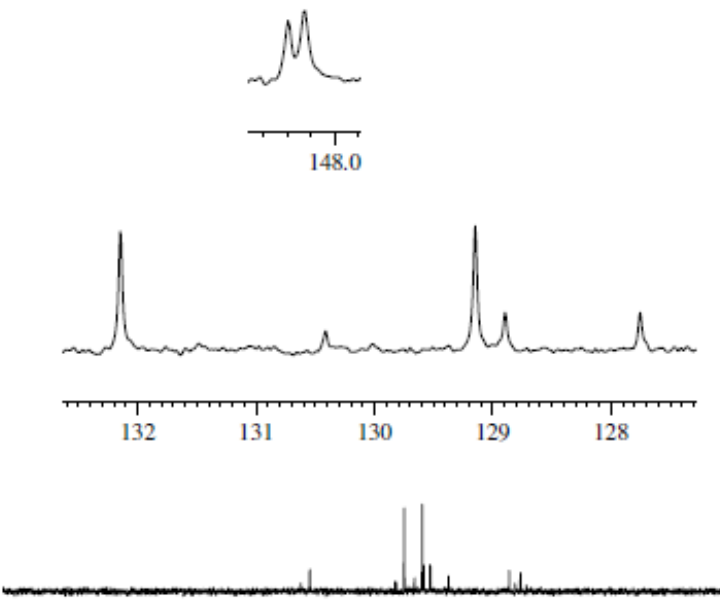

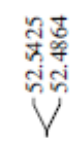

4.
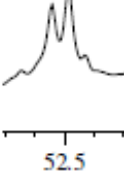

52.5

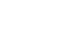

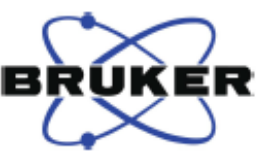

Current Data Parameters EXPNO $\quad 330$

F2 - Acquisition Parameters
Date_ 20160211

$\begin{array}{lc}\text { Date_ } & 20160211 \\ \text { Time } & 14.30\end{array}$

PROBHD 5 mm PABBO BB

PULPROG ${ }_{\text {Zgpg } 30}$

TD ${ }^{65536} \mathrm{CDC13}$

${ }_{\mathrm{NS}}{ }_{512} \mathrm{CDC}$

DS $\quad 0$

$\mathrm{Hz}$

AO $\quad 0.366798 \mathrm{~Hz}$

$\begin{array}{ll}\text { RG } & 201.48 \\ \text { DW } & 20.800 \text { usec }\end{array}$

20.800 usec
6.50 usec

$300.0 \mathrm{~K}$

$200000000 \mathrm{sec}$

$0.03000000 \mathrm{sec}$

$=$ CHANNEL $\mathrm{f1}=$

NUC1

P1 $\quad 9.90 \mathrm{C}$

PLW1 53.00000000 W

CHANNEL $\mathrm{f} 2=$

SFO2 $400.1621006 \mathrm{MHz}$

CPDPRG[2 $1 \mathrm{H}$

$\begin{array}{ll}\text { PCPD2 } & 90.00 \text { usec } \\ & \end{array}$

$\begin{array}{ll}\text { PLW 12 } & 0.27963999 \mathrm{~W} \\ & 0.22651000 \mathrm{~W}\end{array}$

F2 - Processing parameters

FF $\quad 100.6204380 \mathrm{MH}$

EM

$\mathrm{SSB} \quad 0$

$\mathrm{GB} \quad 0-3.00 \mathrm{H}$

$\begin{array}{lllllllllllllllllllll}190 & 180 & 170 & 160 & 150 & 140 & 130 & 120 & 110 & 100 & 90 & 80 & 70 & 60 & 50 & 40 & 30 & 20 & \text { ppmpC } & 0 & 1.40\end{array}$

Figure 8: ${ }^{13} \mathrm{C}$ NMR spectrum of $2 \mathrm{~d}$ 


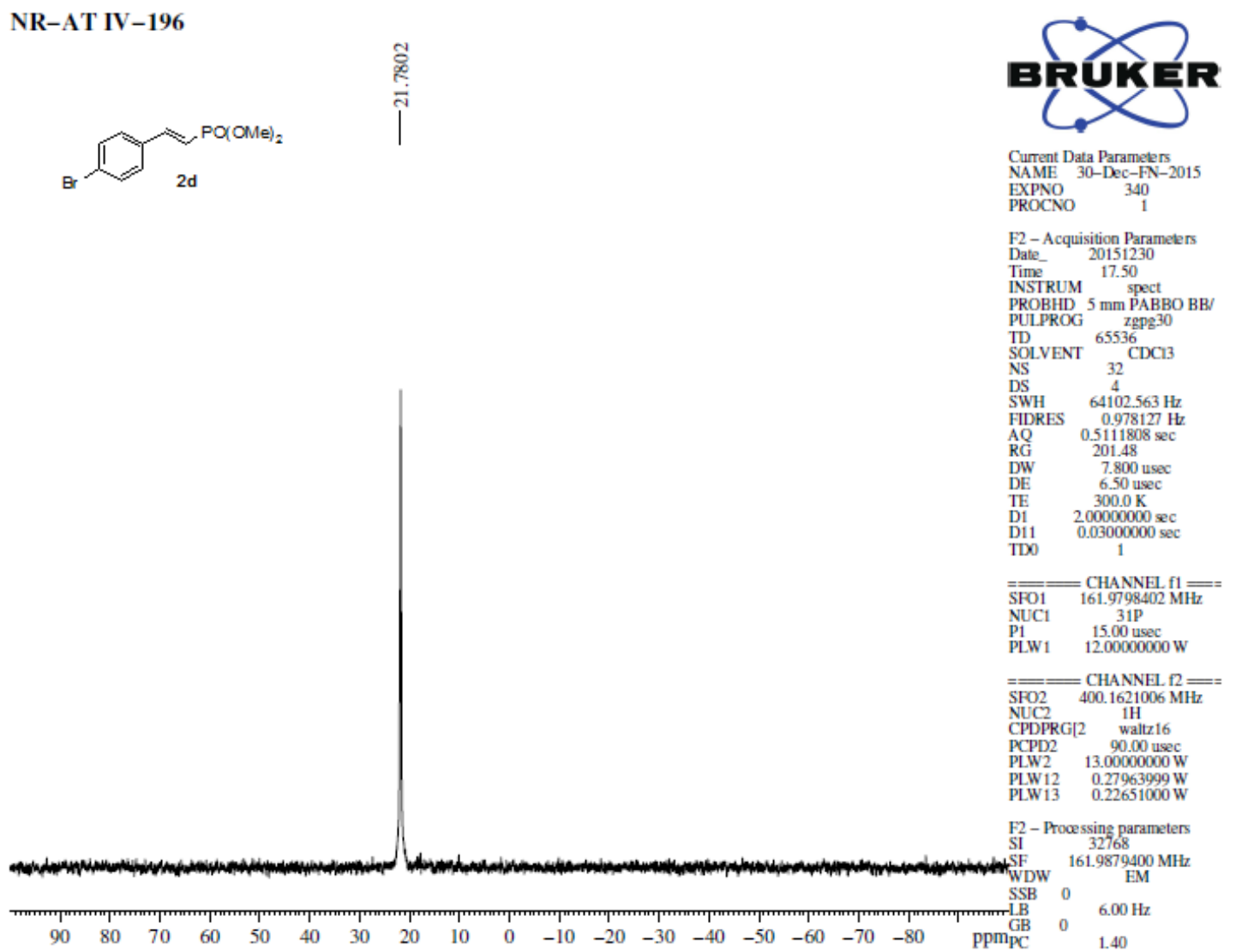

Figure 9: ${ }^{31} \mathrm{P}$ NMR spectrum of $2 \mathrm{~d}$

NR-AT IV-197

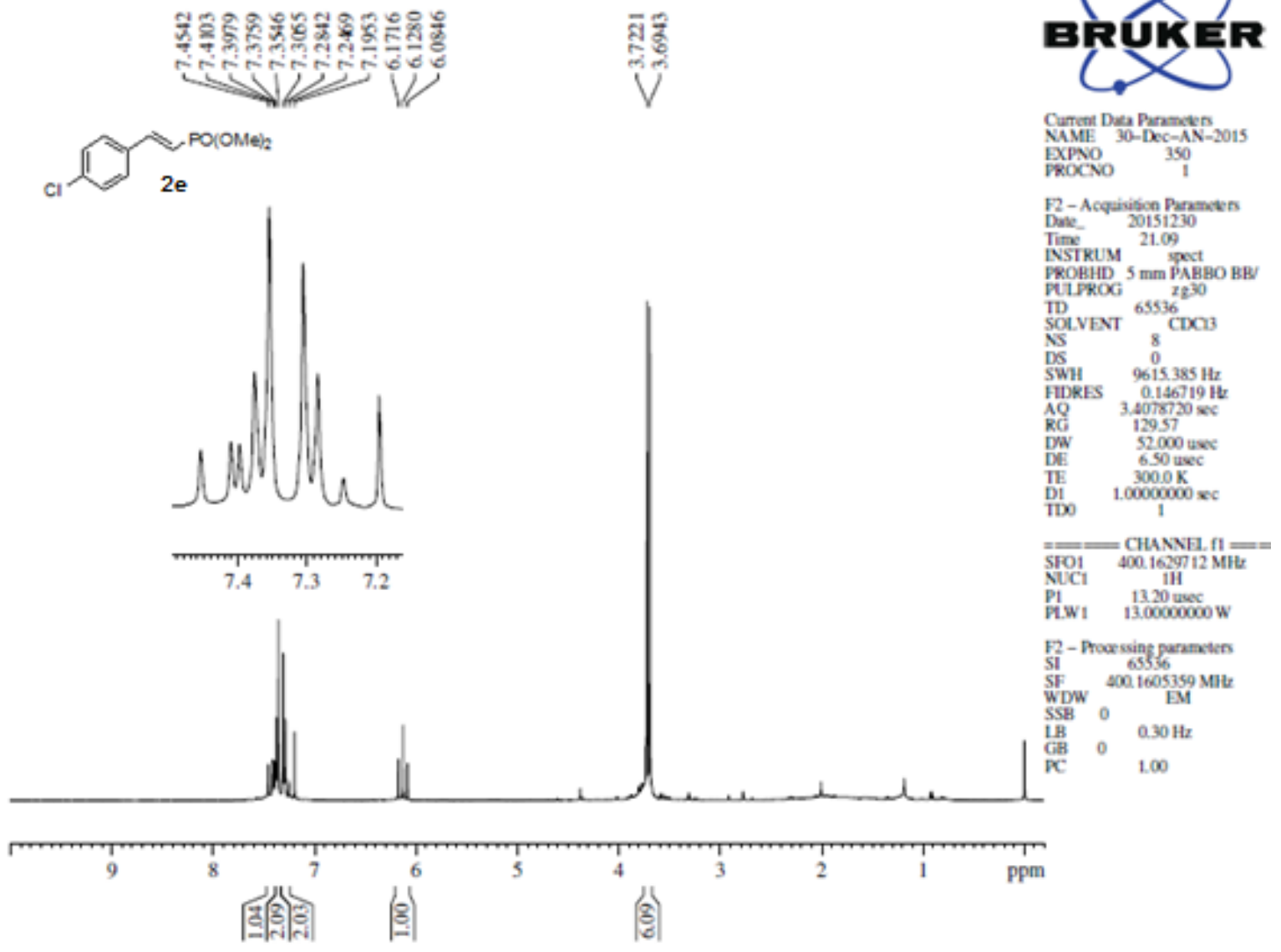

Figure 10: ${ }^{1} \mathrm{H}$ NMR spectrum of $2 \mathrm{e}$ 


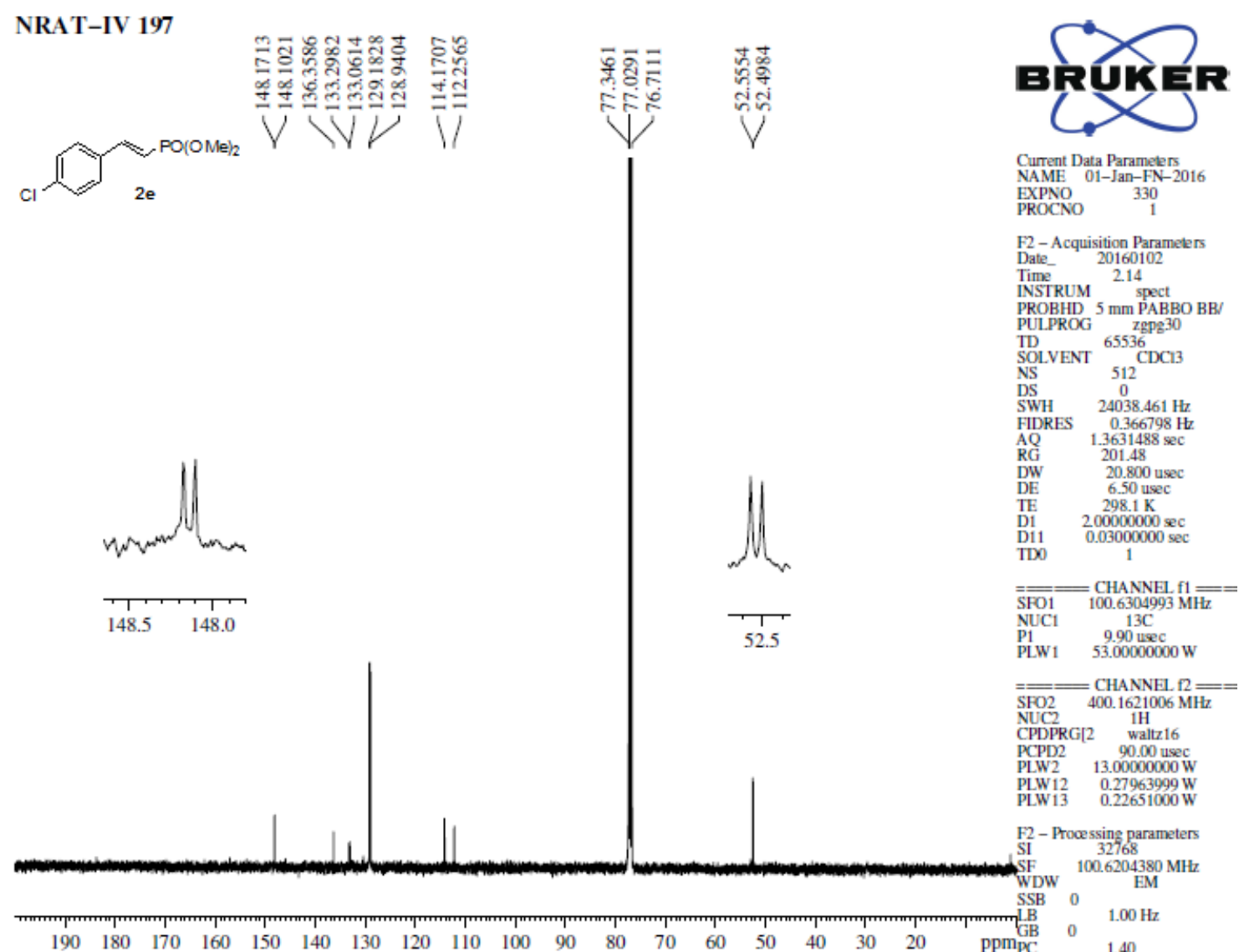

Figure 11: ${ }^{13} \mathrm{C}$ NMR spectrum of $2 \mathrm{e}$

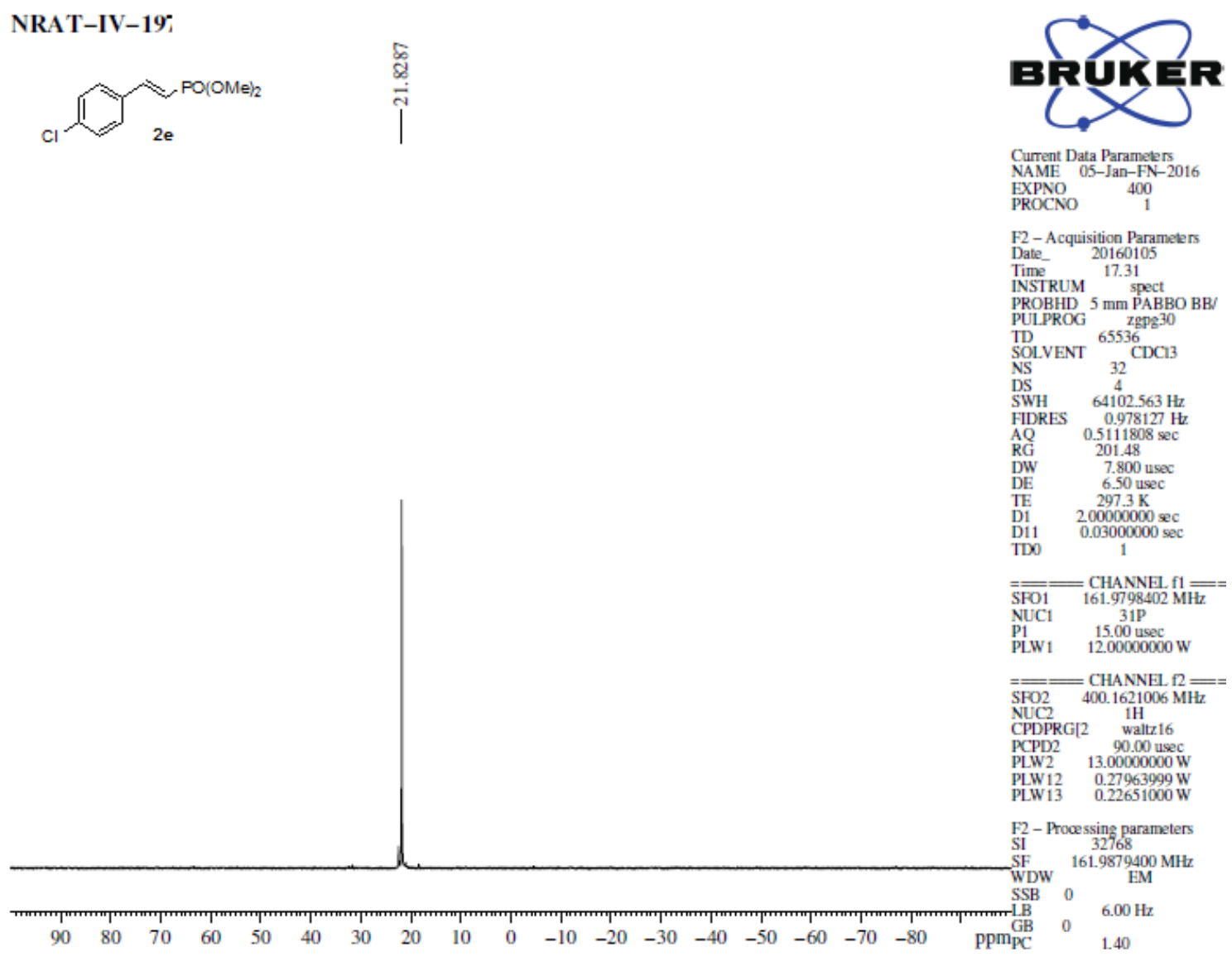

Figure 12: ${ }^{31} \mathrm{P}$ NMR spectrum of $2 \mathrm{e}$ 


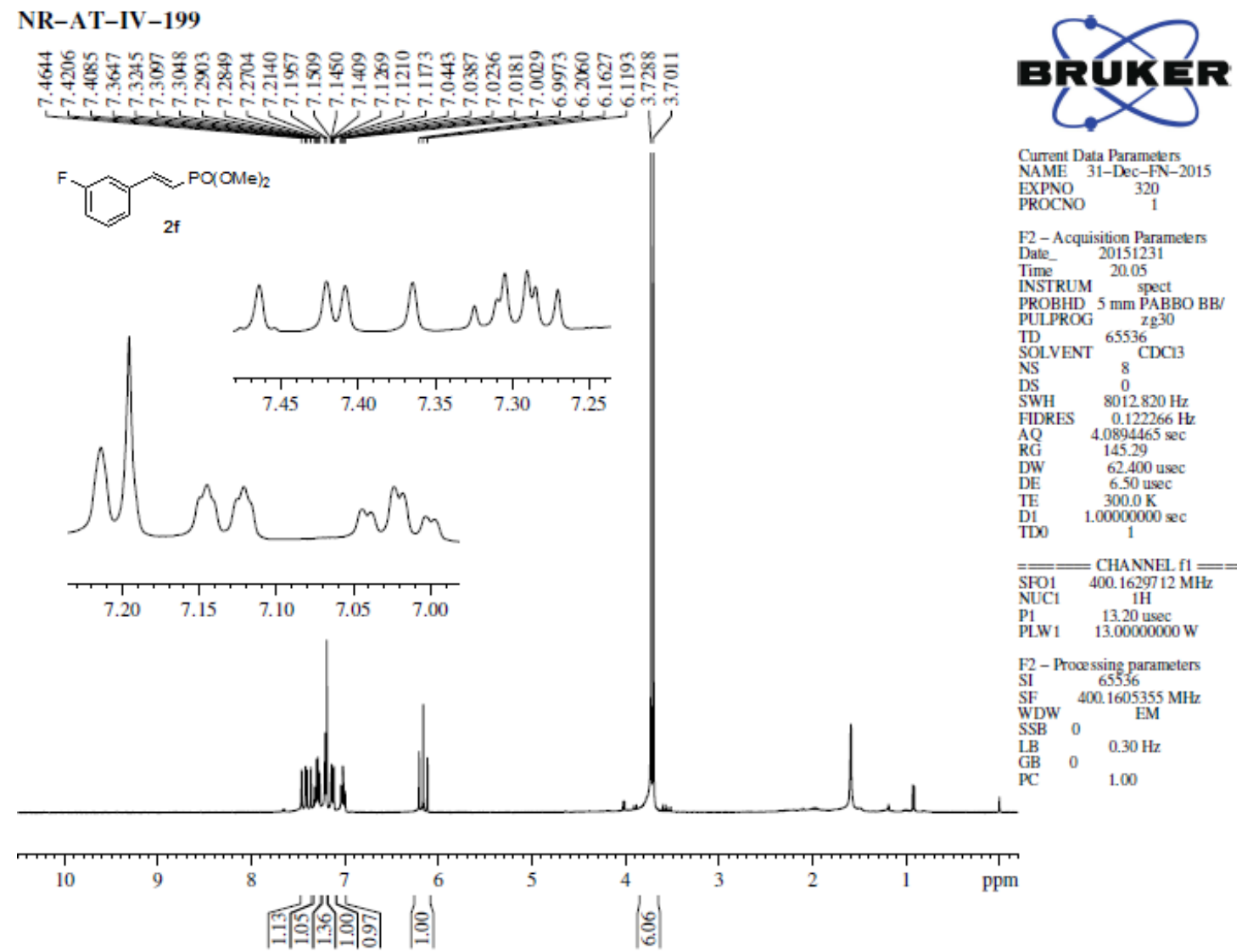

Figure 13: ${ }^{1} \mathrm{H}$ NMR spectrum of $2 \mathrm{f}$

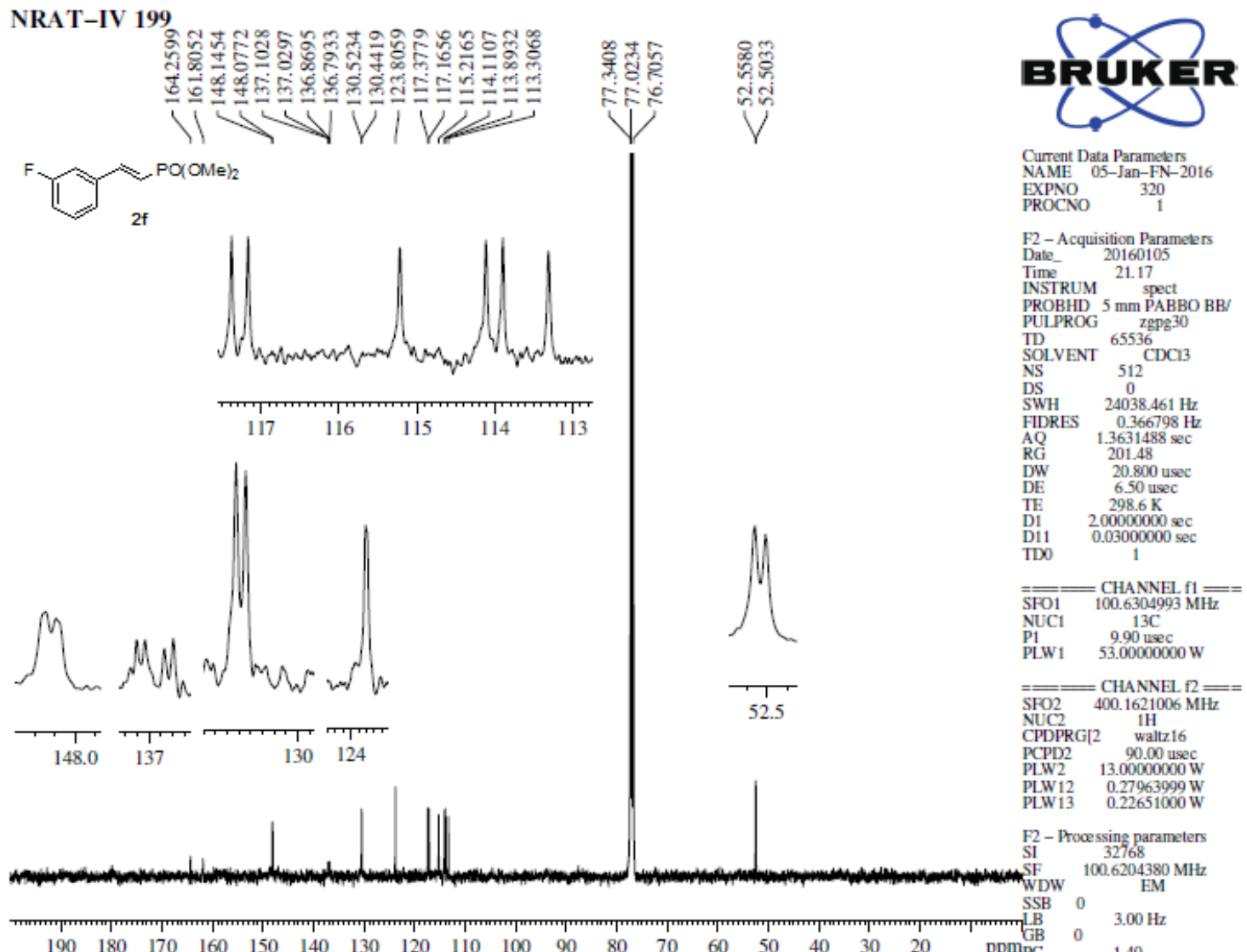

Figure 14: ${ }^{13} \mathrm{C}$ NMR spectrum of $2 \mathrm{f}$ 


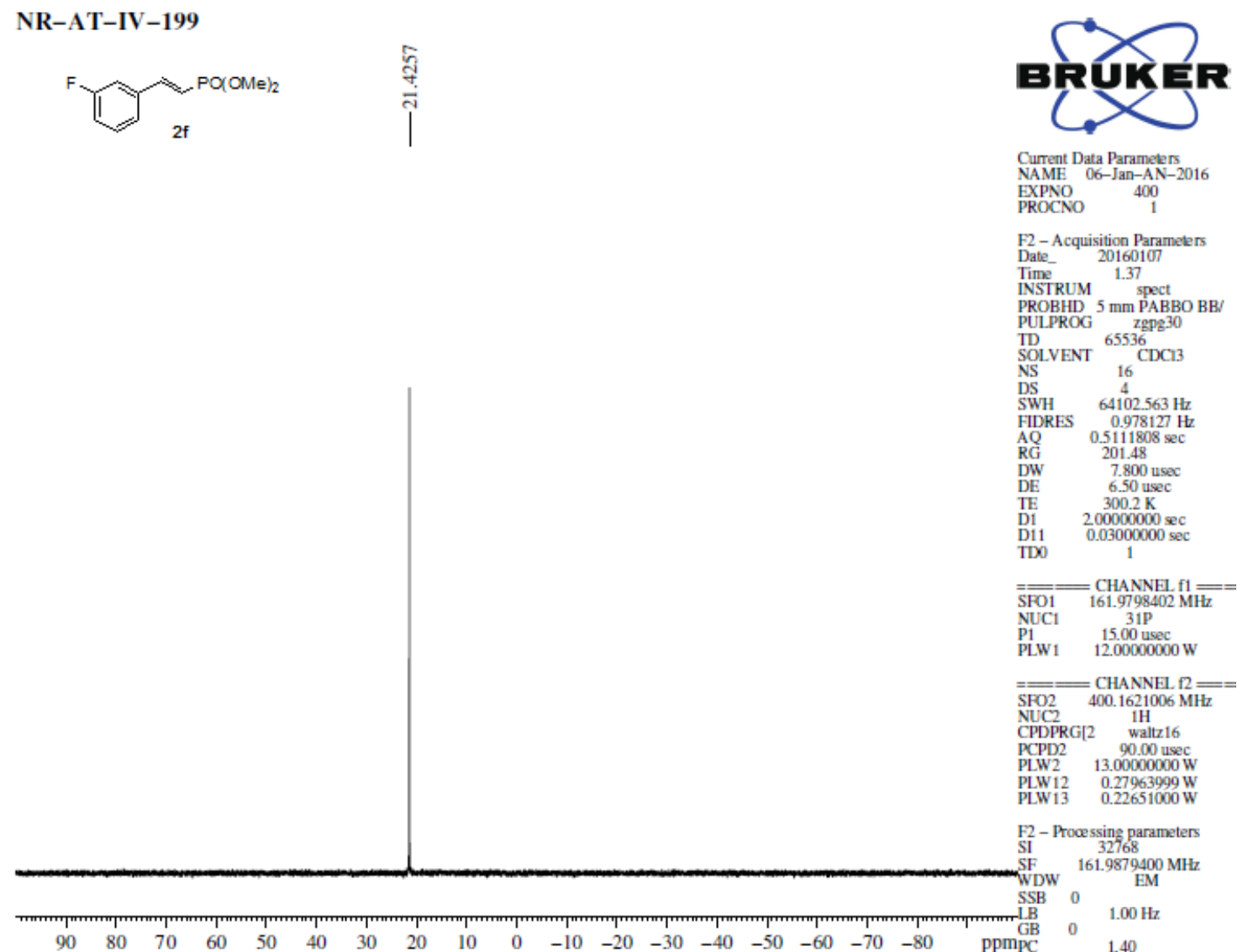

Figure 15: ${ }^{31} \mathrm{P}$ NMR spectrum of $2 \mathrm{f}$

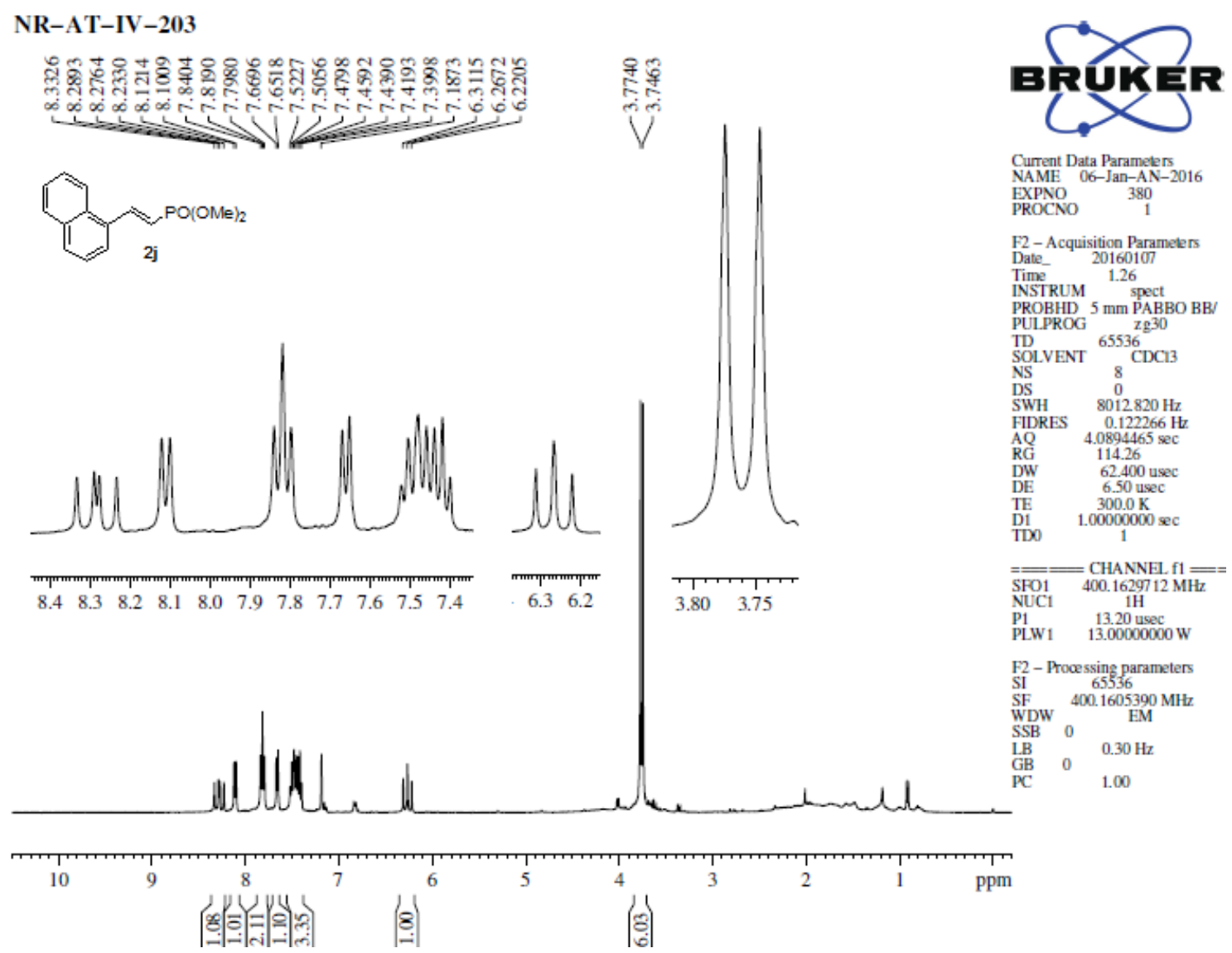

Figure 16: ${ }^{1} \mathrm{H}$ NMR spectrum of $2 \mathrm{j}$ 


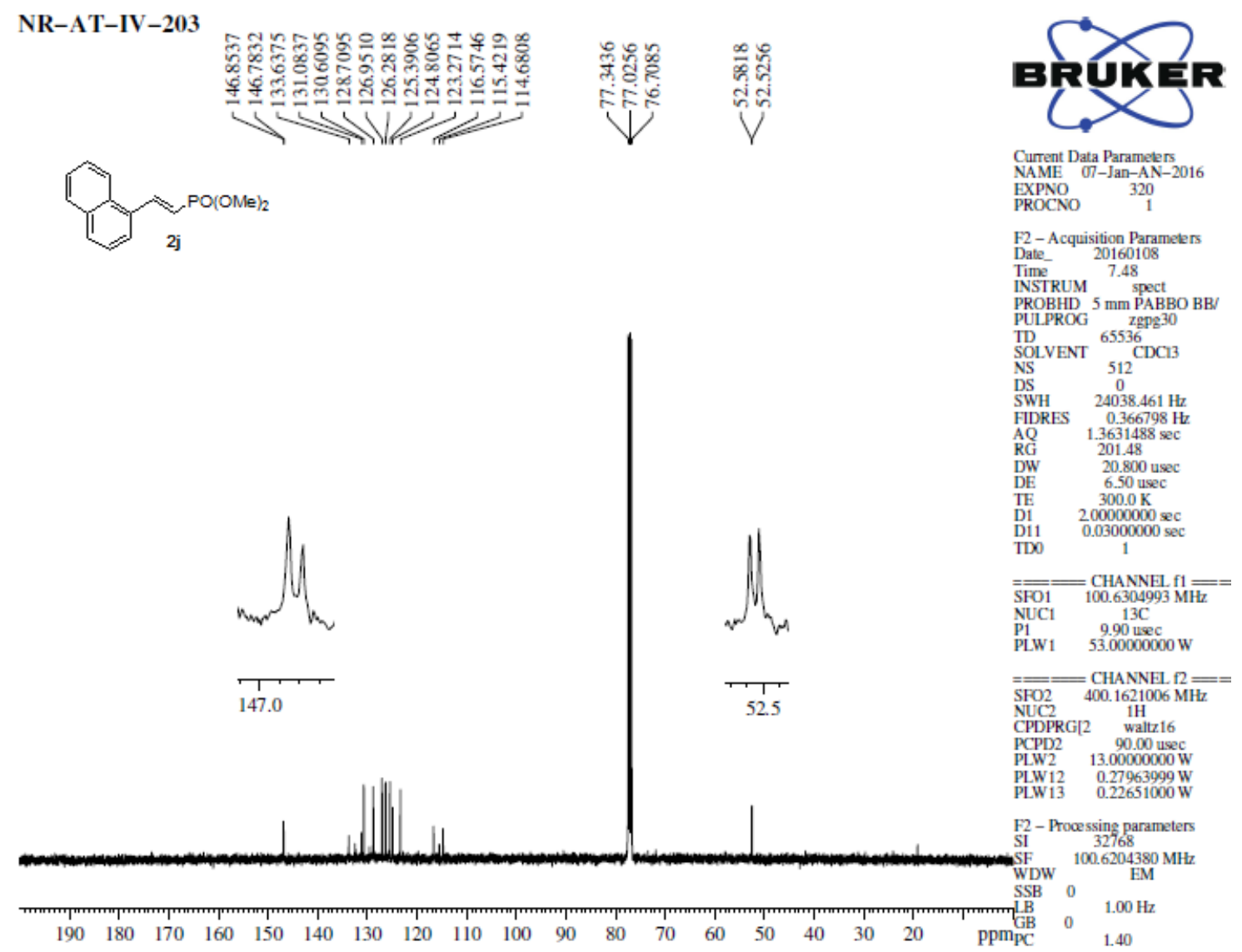

Figure 17: ${ }^{13} \mathrm{C}$ NMR spectrum of $2 \mathrm{j}$
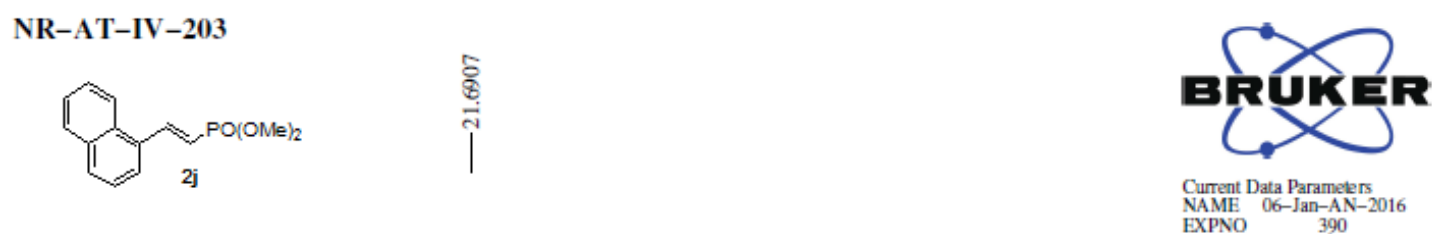

EXPNO $\quad 390$

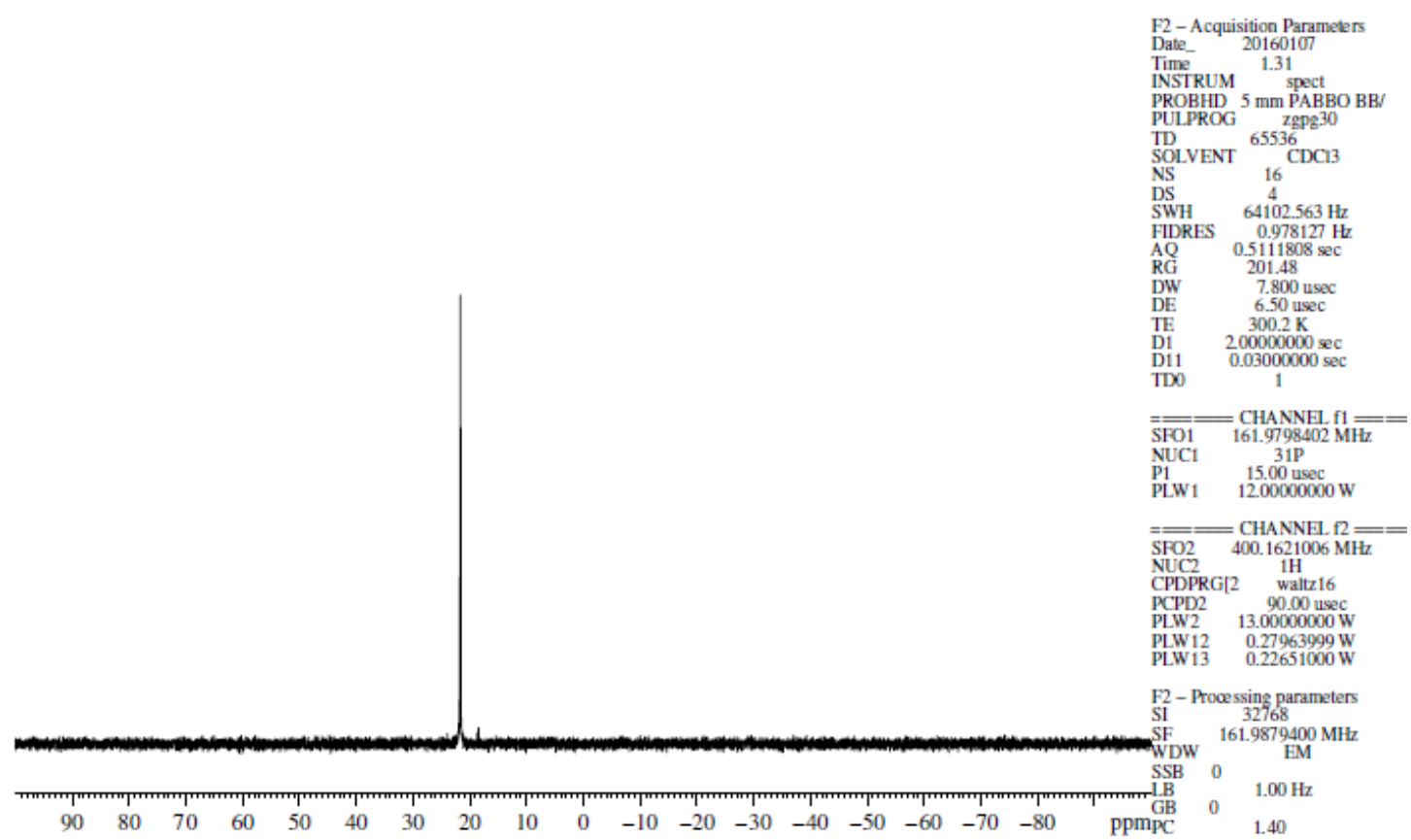

Figure 18: ${ }^{31} \mathrm{P}$ NMR spectrum of $2 \mathrm{j}$ 


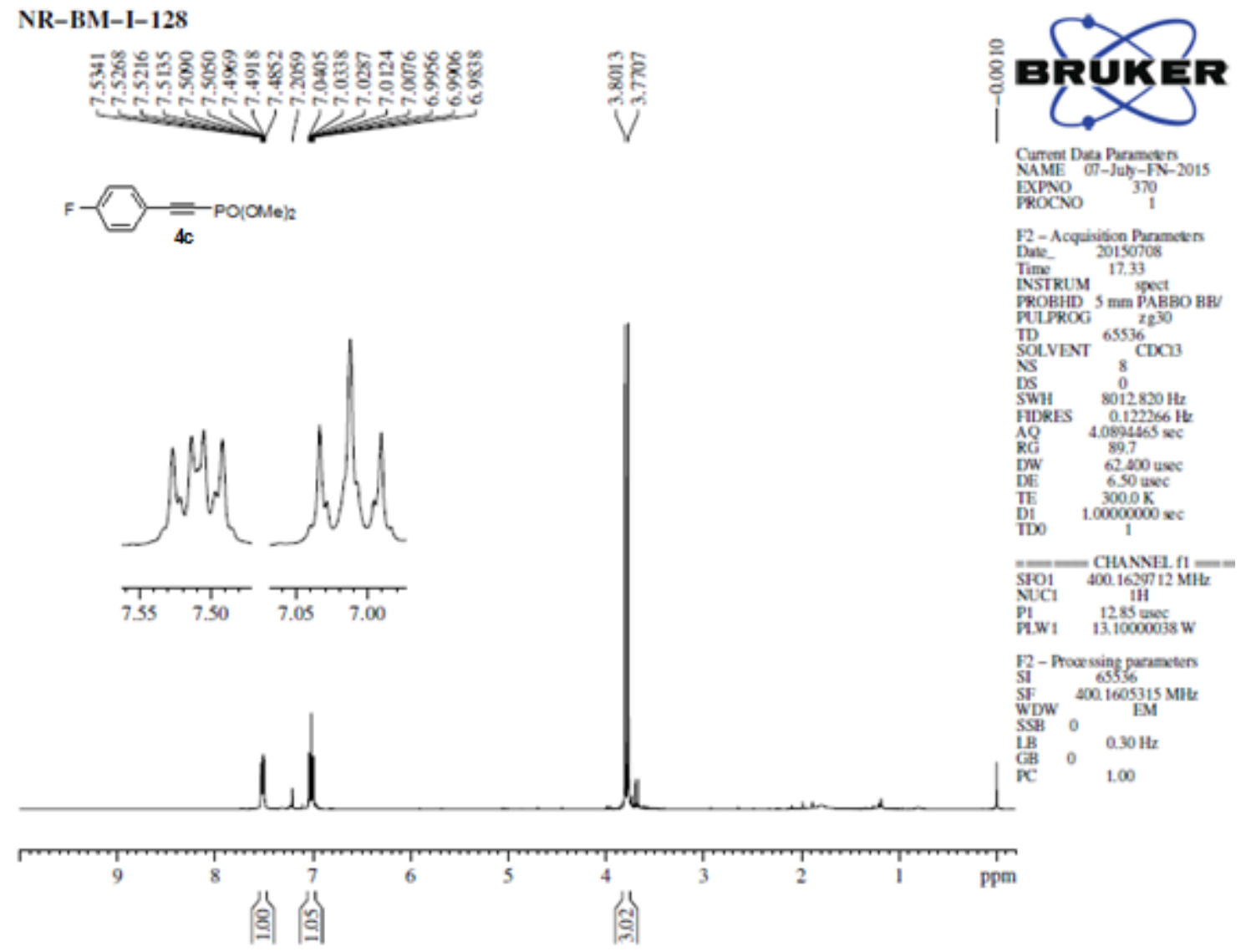

Figure 19: ${ }^{1} \mathrm{H}$ NMR spectrum of $4 \mathrm{c}$

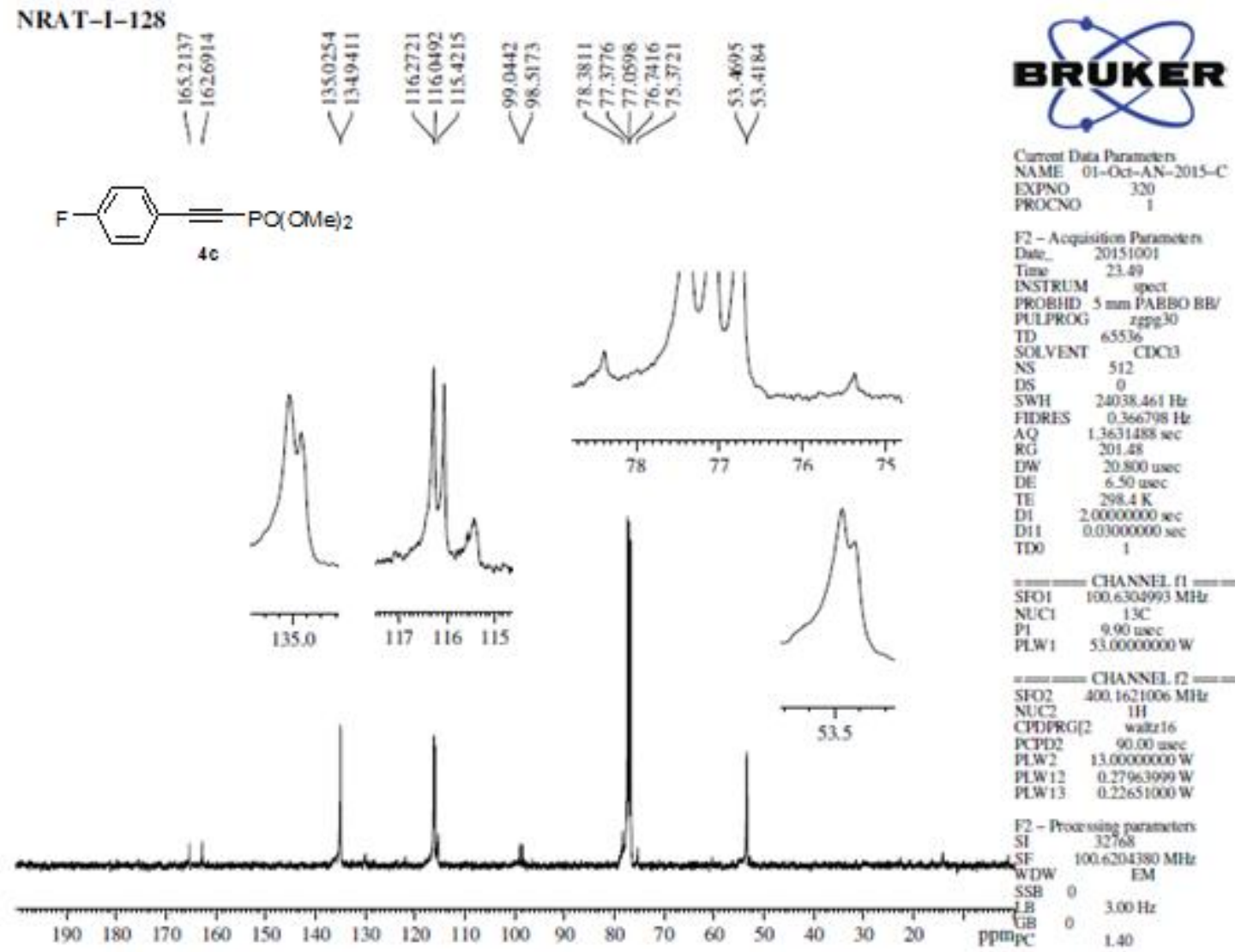

Figure 20: ${ }^{13} \mathrm{C}$ NMR spectrum of $4 \mathrm{c}$ 


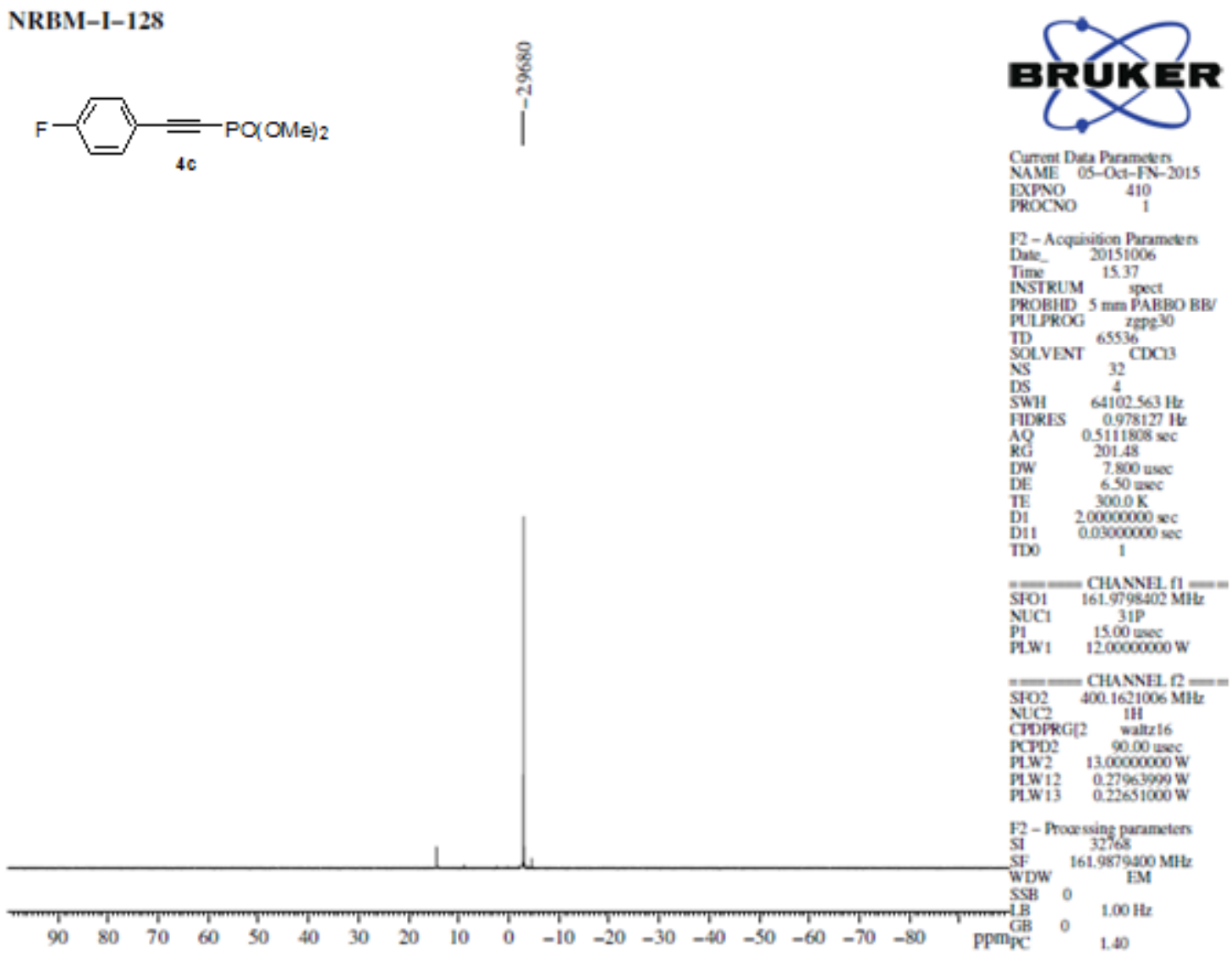

Figure 21: ${ }^{31} \mathrm{P}$ NMR spectrum of $4 \mathrm{c}$

NRAT-IV-49

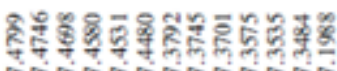

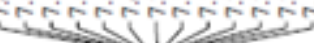

Bd $=$ Poromeis
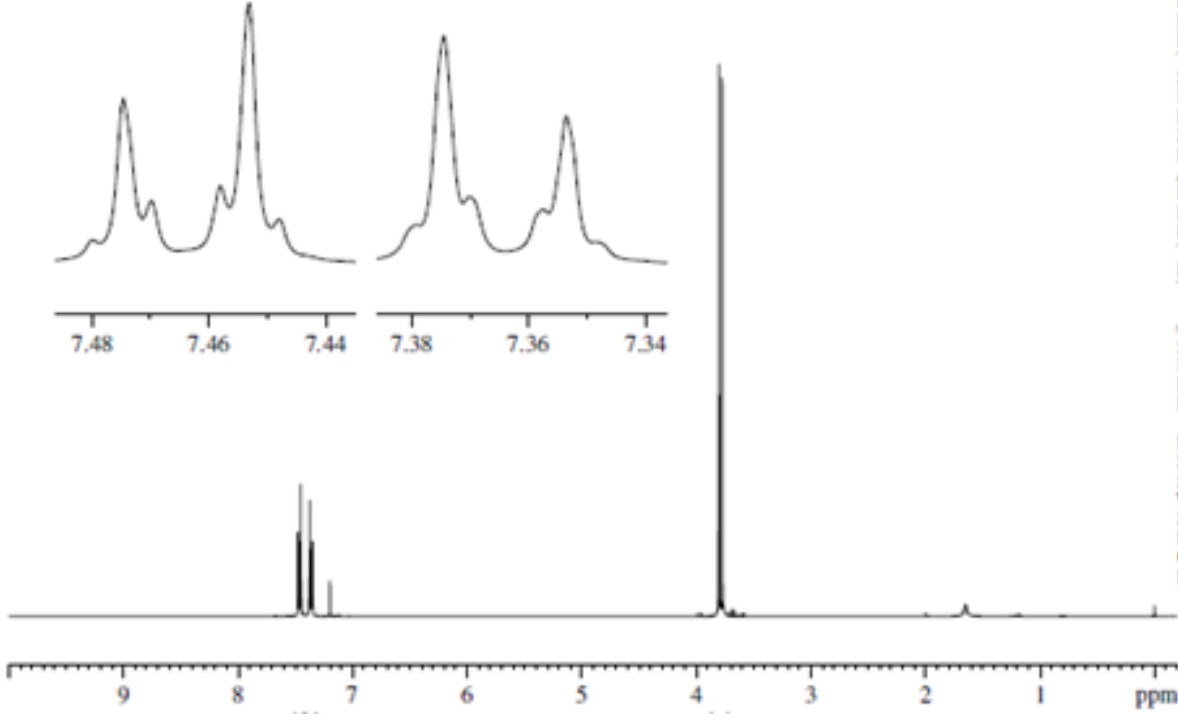

의흐

|

Figure 22: ${ }^{1} \mathrm{H}$ NMR spectrum of $4 \mathrm{~d}$ 


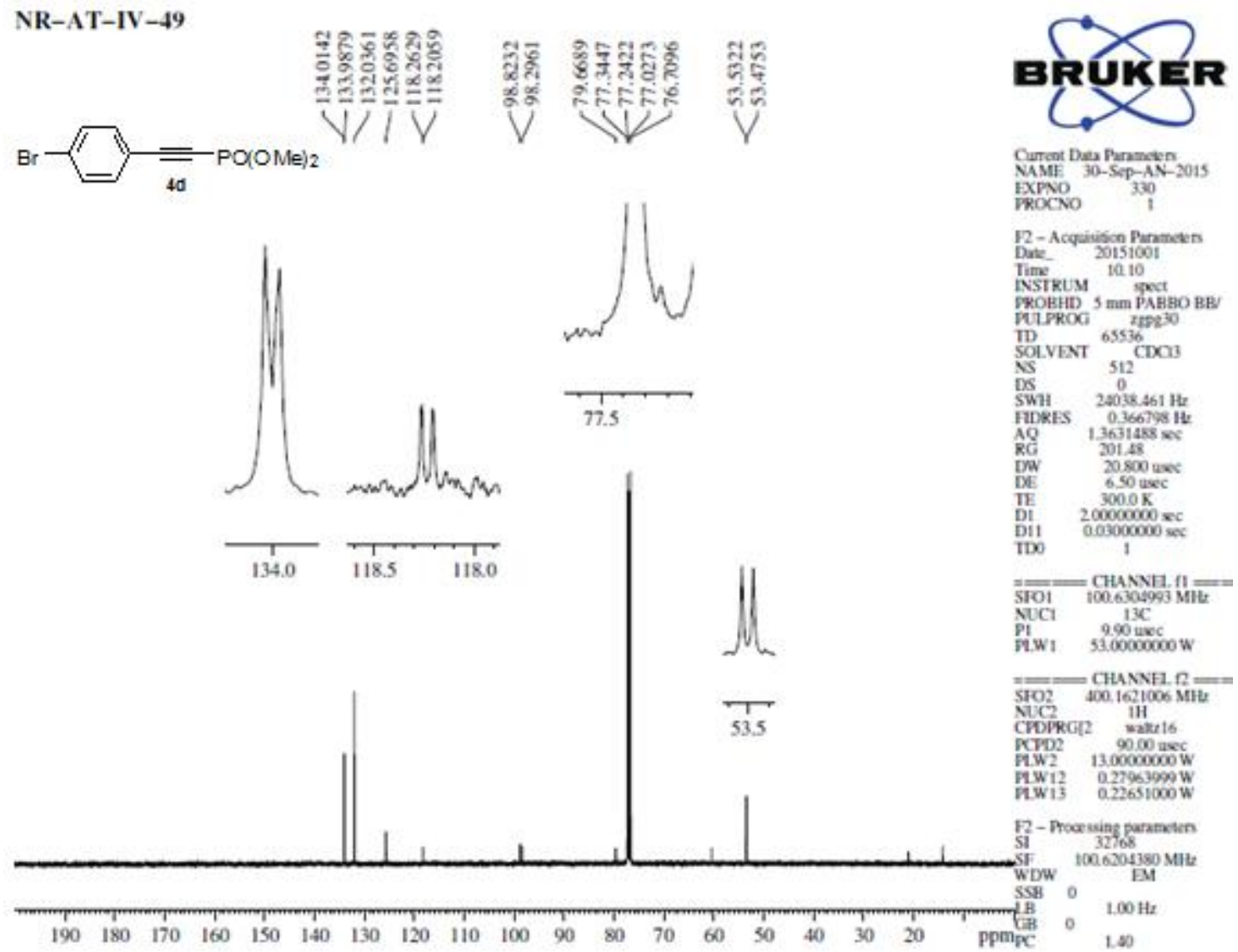

Figure 23: ${ }^{13} \mathrm{C}$ NMR spectrum of $4 d$

NR-AT-IV-49
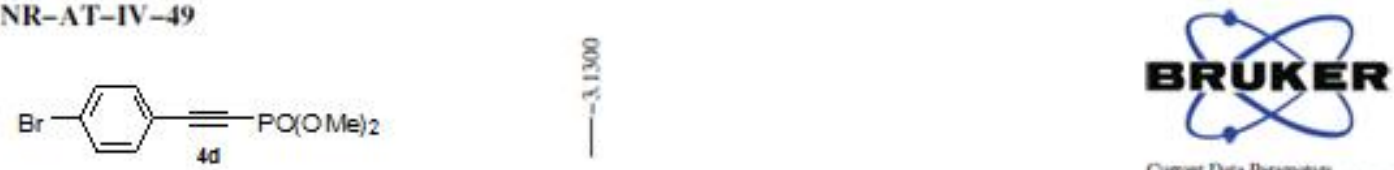

Ninget Daz Prandes

EXNO

F2-Acquibion Pramenten

Done. 30151001

NSTREM Mect

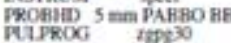

ID

IDOAVEST ${ }^{65316} \mathrm{CDCO}$

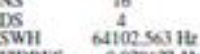

HDRES 0.978127

RG 201.48

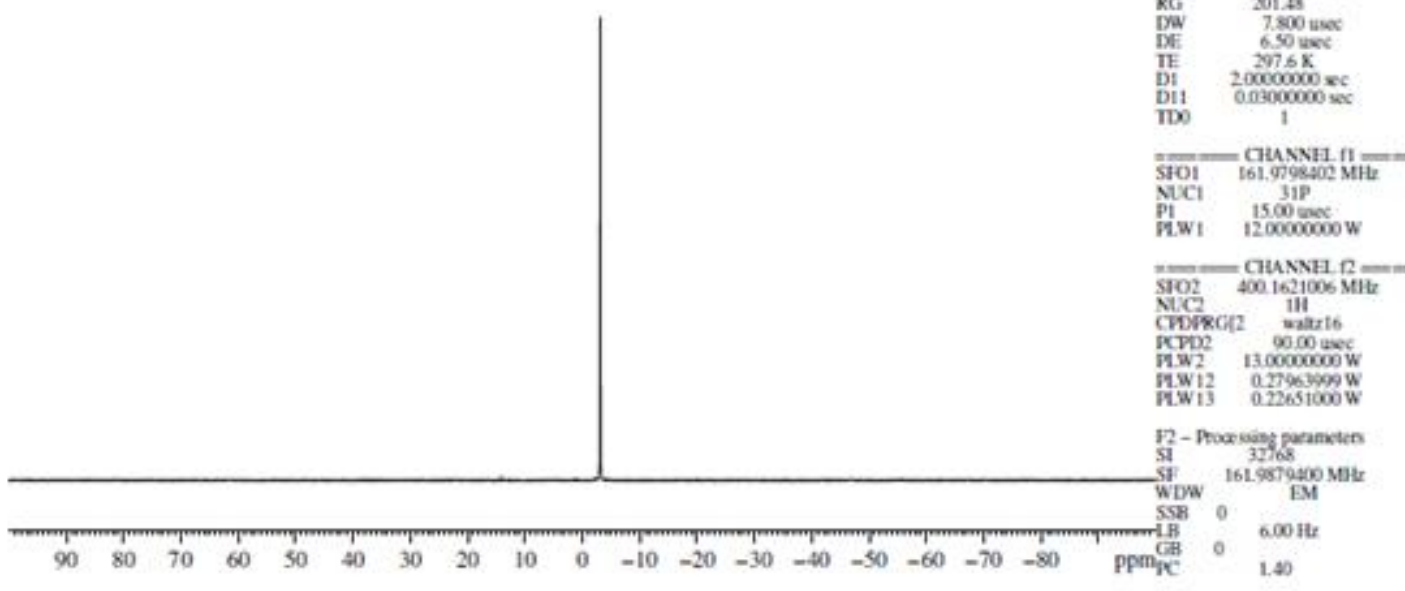

Figure 24: ${ }^{31} \mathrm{P}$ NMR spectrum of $4 \mathrm{~d}$ 


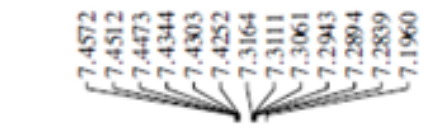

$\mathrm{CI}=-\frac{\overline{\bar{a}}}{4 \mathrm{e}}$ polomels
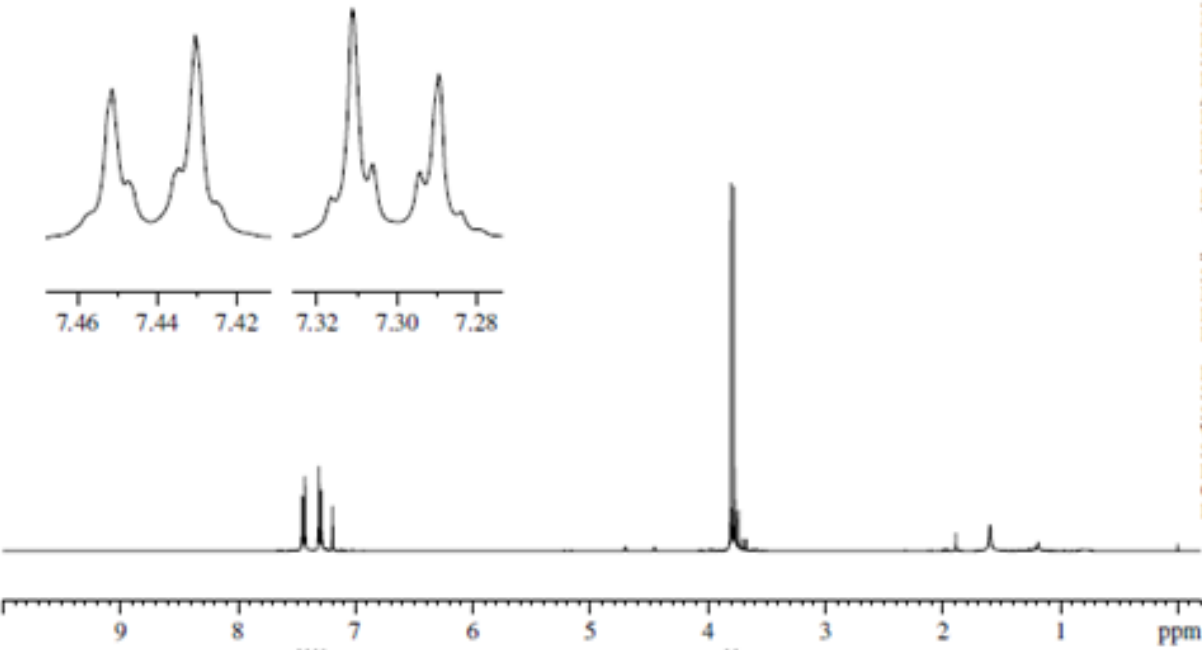

현일

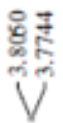

永

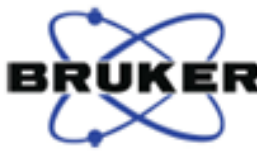

Currese Dasa Parametss EXPNo 330

F2-Acequivisce Parameves

Dime 7,48

INSTRUM spect

PROBHID 5 mm PABBO

TDOLVENT ${ }^{65536} \mathrm{CDCO}$

SOLVENT CDC

DS 001289

HDRES $0.1220 \times 6$ He

AO $4089465 \times$

RG $\quad 145.29$

DE $\quad 6.50 \mathrm{axec}$

IE $\quad 300.0 \mathrm{~K}$

ID0 1000000

$=\underset{401}{=}$ CHANNH. $\mathrm{fl}=$

PI 12.85 usec

F2 - Prowssing parameten

Si $\quad 05536$. 500.1605355 MIt

IB $0.30 \mathrm{~Hz}$

1.00

Figure 25: ${ }^{1} \mathrm{H}$ NMR spectrum of $4 \mathrm{e}$

NR-BM-105
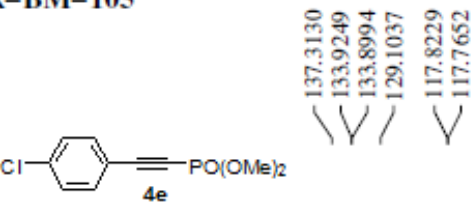

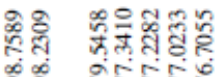

윰ํㅇ

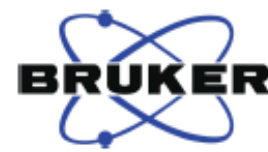

Current Data Parameters

EXPNO

F2 - Acquisition Parameters
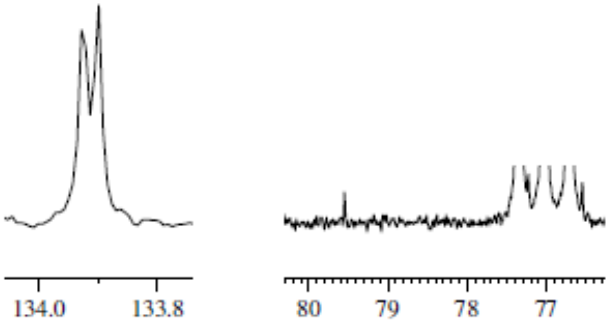

Time 3.07

PROBHD 5 mm PABBO BB/

PULPROG ZFPPB 30

TD ${ }_{\text {SOLVENT }}^{65536}{ }^{\mathrm{CDC13}}$

NS 512 CDCI3

DS

$\begin{array}{ll}\text { DS } & 0 \\ \text { SWH } & 24038.461 \mathrm{~Hz}\end{array}$

FIDRES $\quad 0.366798 \mathrm{H}$

AQ $\quad 1.3631488 \mathrm{sec}$

$\begin{array}{ll}\text { RG } & 201.48 \\ \text { DW } & 20.800 \text { usec }\end{array}$

DE $\quad 6.50$ usec

TE $\quad 300.0 \mathrm{~K}$

Di1 $0.03000000 \mathrm{sec}$

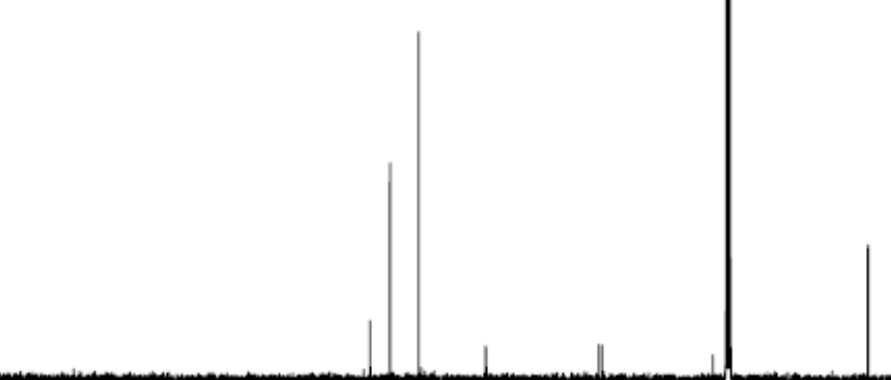

$3000000 \mathrm{sec}$

$=\overline{\text { CHANNEI }} \mathrm{f1}=$

$\begin{array}{lc}\text { SFO1 } & 100.6304993 \\ \text { NUC1 } & 13 \mathrm{C}\end{array}$

$\begin{array}{ll}\text { PLW1 } & 9.90 \text { usec } \\ \text { PL.00000000 W }\end{array}$

$=$ CHANNEL $\mathrm{f} 2=$

FO2 $400.1621006 \mathrm{MH}$

CPDPRG12 waltz16

PCPD2
PLW2 $13000000000 \mathrm{wsec}$

PLW2 $13.00000000 \mathrm{~W}$

$\begin{array}{ll}\text { PLW 12 } & 0.27963999 \mathrm{~W} \\ \text { PLW 13 } & 0.22651000 \mathrm{~W}\end{array}$

F2 - Processing parameters

SF $100.6204380 \mathrm{MHz}$

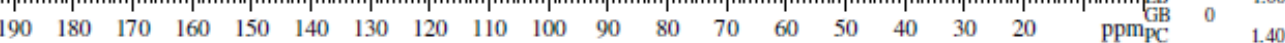

Figure 26: ${ }^{13} \mathrm{C}$ NMR spectrum of $4 \mathrm{e}$ 
NR-BM-105

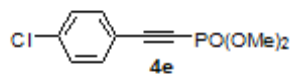

Current Data Parameters

XPNO

PROCNO

F2 - Acquisition Parameers

Time- 20.38

INSTRUM spect

PROBHD $5 \mathrm{~mm}$ PABBO BB

PUDPPB 30

${ }_{\text {SOLVENT }}^{\text {TD }}{ }^{65536}$ CDC13

NS $\quad 16$

$\begin{array}{lll}\text { DS } & 4 \\ \text { SWH } & 64102.563 \mathrm{~Hz}\end{array}$

FIDKES $0.978127 \mathrm{HE}$

${ }_{\text {AG }}^{A} \quad 0.5111808$

$\begin{array}{ll}\text { DW } & 7.800 \text { usec } \\ \text { DE } & 6.50 \text { usec }\end{array}$

TE $297.7 \mathrm{~K}$

$\begin{array}{ll}\text { Di } & 200000000 \mathrm{sec} \\ \text { Di1 } & 0.03000000 \mathrm{sec}\end{array}$

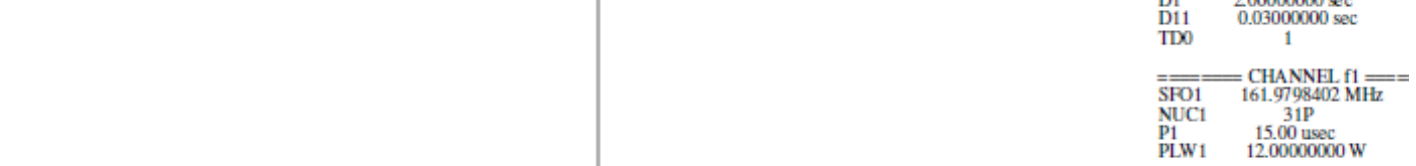

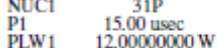

$=\equiv$ CHANNEL $12=$

SFO2 $400.1621006 \mathrm{MH}$

CPDPRG|2 14 wallz16

$\begin{array}{ll}\mathrm{PCPD} 2 & 90.00 \text { usec } \\ & \end{array}$

F2 - Processing parameters

$\underset{\text { WDW }}{\mathrm{SI}} \begin{gathered}161.9879400 \mathrm{MHz} \\ \mathrm{EM}\end{gathered}$

SB 00

$\begin{array}{lllllllllllllllllllllll}90 & 80 & 70 & 60 & 50 & 40 & 30 & 20 & 10 & 0 & -10 & -20 & -30 & -40 & -50 & -60 & -70 & -80 & \operatorname{ppm}_{\mathrm{GC}} & 0 & 1.40\end{array}$

Figure 27: ${ }^{31} \mathrm{P}$ NMR spectrum of $4 \mathrm{e}$

NR-BM-113

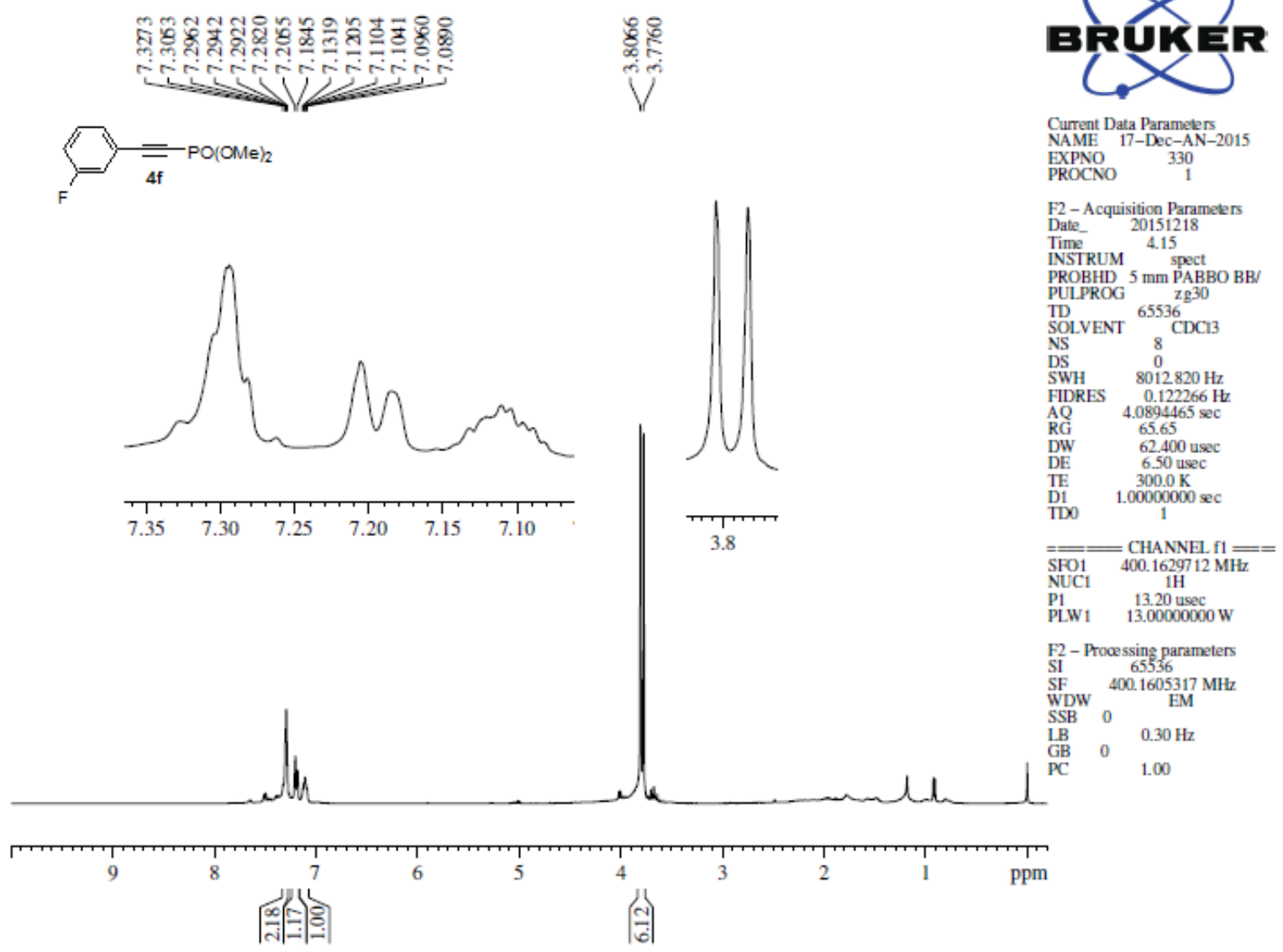

Figure 28: ${ }^{1} \mathrm{H}$ NMR spectrum of $4 f$ 
<smiles>COC#Cc1cccc(F)c1</smiles>
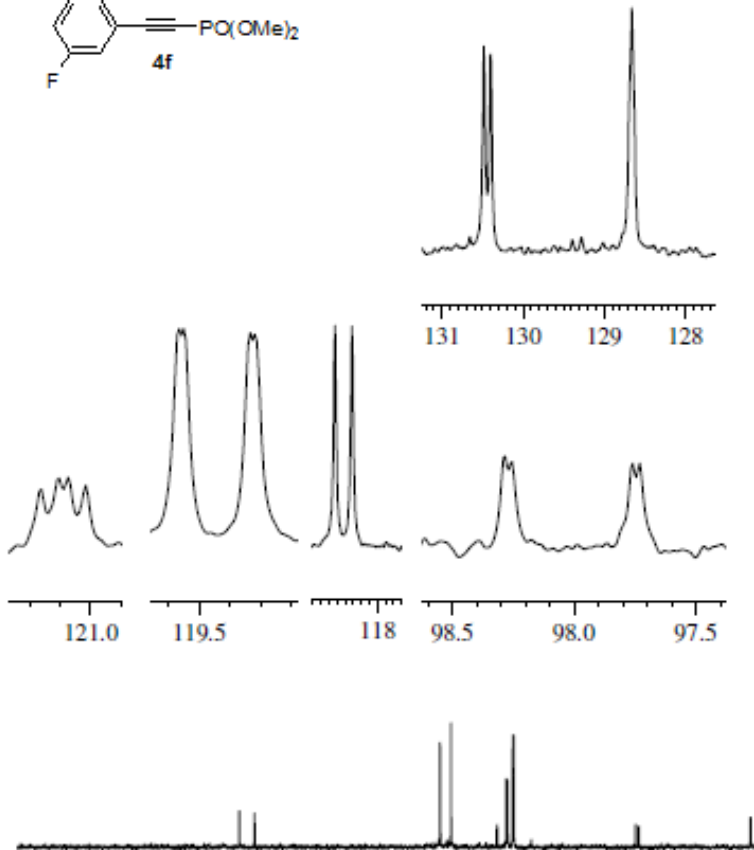
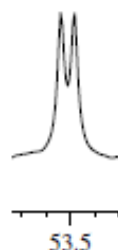

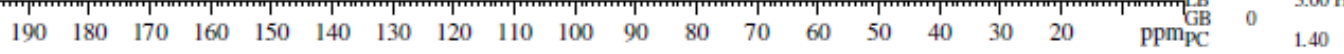

\section{Figure 29: ${ }^{13} \mathrm{C}$ NMR spectrum of $4 f$}

NR-BN-113

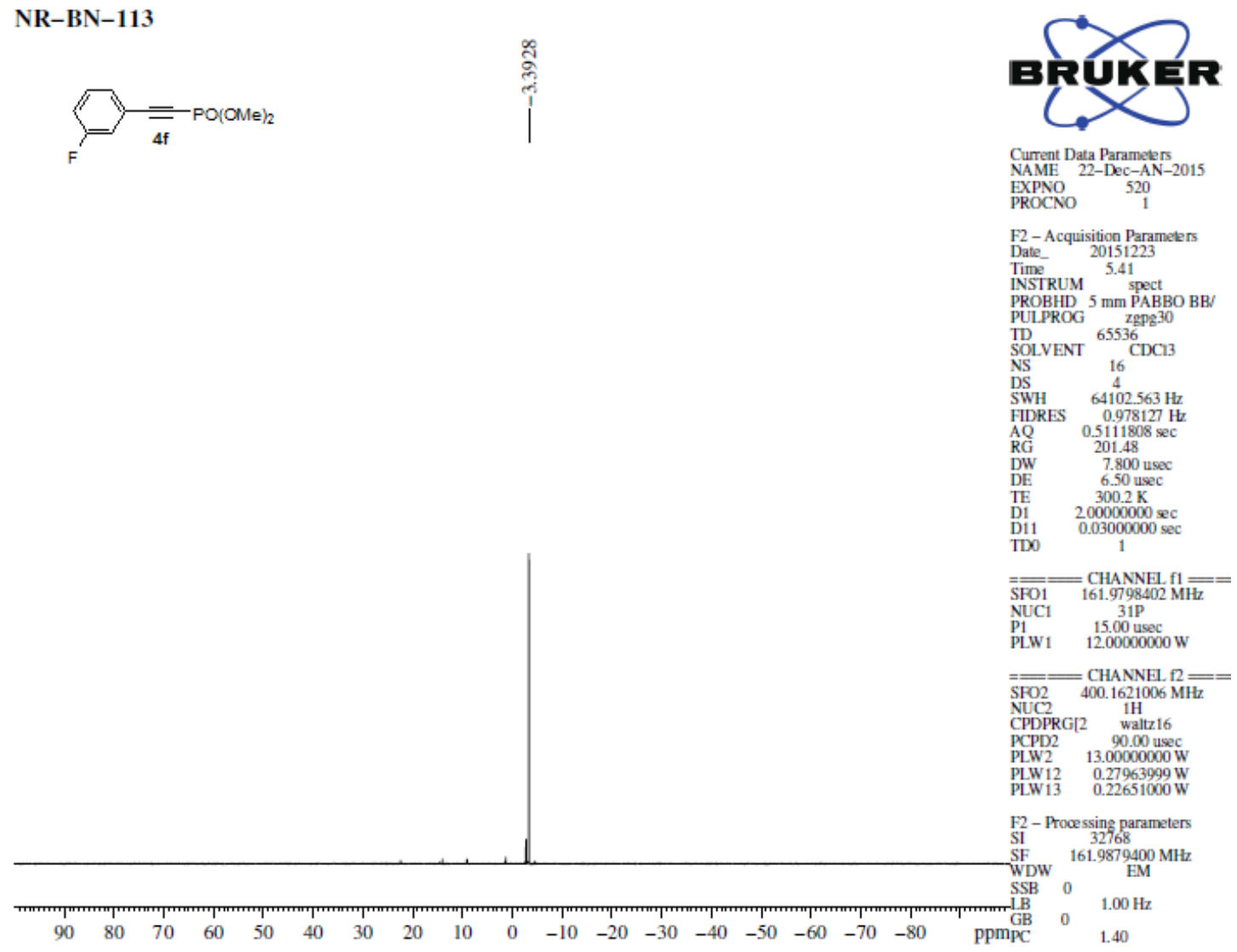

D2-Acquisition Parameter

Time

PROBHD 5 mm PABBO BB/

${ }_{\text {PULPROG }} 5$ maps PABBO

TD 65536

NS 512 CDCi3

DS

SWH $\quad 24038.461 \mathrm{~Hz}$

FIDRES $0.366798 \mathrm{H}$

$\begin{array}{ll}\text { AQ } & 1.3631488 \\ \text { RG } & 201.48\end{array}$

DW $\quad 20.800$ usec

TE $\quad 300.0 \mathrm{~K}$

$\begin{array}{ll}\text { D1 } & 200000000 \mathrm{sec} \\ \text { D11 } & 0.03000000 \mathrm{sec}\end{array}$

DI1 $0.03000000 \mathrm{sec}$

$\overline{\overline{S F O} 1}={ }_{100.6304993}$ CHANNE

NUC1 $13 \mathrm{C}$

$\begin{array}{ll}\text { P1 } & 9.90 \mathrm{usec} \\ \text { PLW1 } & 53.00000000 \mathrm{~W}\end{array}$

$\underset{\text { SFO2 }}{=}=\underset{\substack{400.1621006 \mathrm{MHz} \\ \text { CHANE }}}{\text { CHA }}=$

CPDPRG[2 waltz16

$\begin{array}{ll}\text { PCPD2 } & 90.000 \mathrm{usec} \\ \text { PLW2 } & 13.00000000 \mathrm{~W}\end{array}$

LW12 $0.27963999 \mathrm{~W}$

$\underset{\text { S1 }}{\text { F2 - Processing parameters }} 32768$

SF
WDW
$100.6204380 \mathrm{MH}$

SB 0

40

Figure 30: ${ }^{31} \mathrm{P}$ NMR spectrum of $4 \mathrm{f}$ 


\section{NRBM-154}

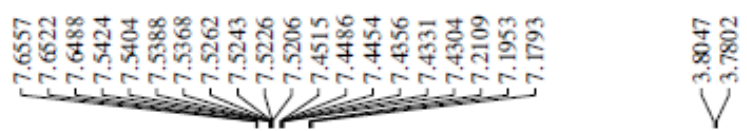

${ }_{\mathrm{Br}}=\frac{}{4 \mathrm{~g}} \mathrm{PO}(\mathrm{OMe})_{2}$
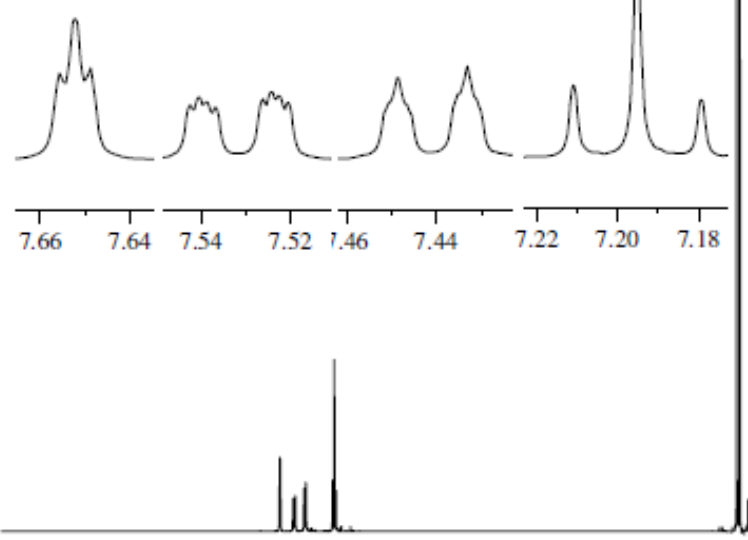

ll

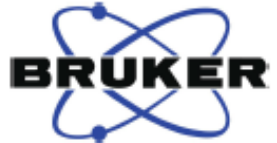

Current Data Paramelers
NAME 02-Feb-FN-2016

EXPNO

F2 - Acquisition Parameters

F2 - Acquisition Para
Date_ 20160202

Time 7.47

INSTRUM spect

PROBHD 5 mm PATXI 1

PULPROG $2 \mathrm{~g} 30$

TD $_{\text {SOLVENT }}{ }^{65536}$ CDCI3

NS 8

DS $\quad 12019.230 \mathrm{~Hz}$

FIDRES $\quad 0.183399 \mathrm{H}$

AQ $\quad 2.7262976 \mathrm{sec}$

$\begin{array}{ll}\text { RG } & 201.78 \\ \text { DW } & 41.600 \text { use }\end{array}$

DE $\quad 6.50 \mathrm{usc}$

D1 $1.00000000 \mathrm{sec}$

$=\overline{=}$ CHANNEL $\mathrm{f} 1=$

SFO1 $500.6794419 \mathrm{MHz}$

$\begin{array}{lc}\text { P1 } & 6.45 \text { usec } \\ \text { PLW1 } & 13.60000038 \mathrm{~W}\end{array}$

F2 - Prowssing parameter

SI $\quad 65536$

SF $\quad 500.6763941 \mathrm{MH}$

SSB 0 EM

$\mathrm{LB} \quad 0.30 \mathrm{H}$

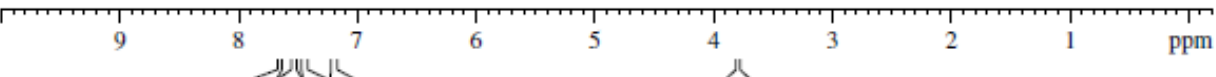

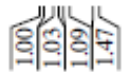

Figure 31: ${ }^{1} \mathrm{H}$ NMR spectrum of $4 \mathrm{~g}$

NR-BM-154
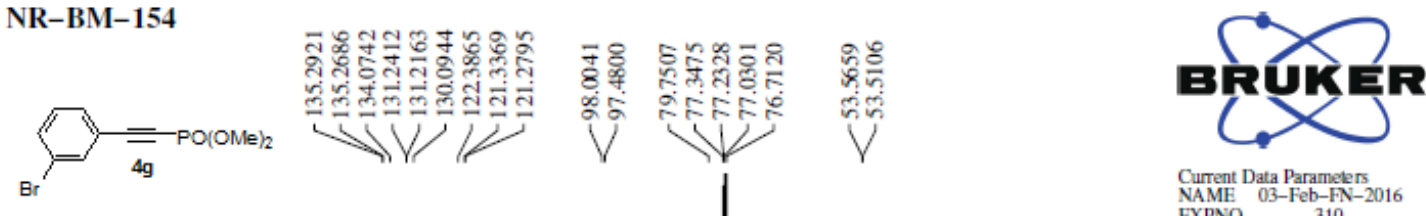

Current Data Parameters
NAME 03-Feb-FN-2016

$\begin{array}{ll}\text { EXPNO } & 310 \\ \text { PROCNO } & 1\end{array}$

F2-Acquisition Parameters

Date_ 2015100

Time 9.03

PROBHD 5 mm PABBO BB

PULPROG ${ }_{\text {zgpg } 30}$

ID 65536

SOLVENT ${ }_{512} \mathrm{CDCl}$
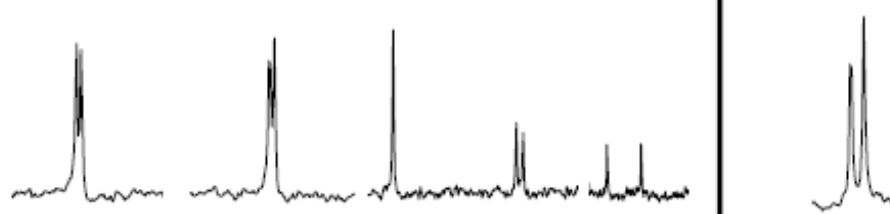

DS $\quad 00$

AQ $\quad 1.3631488 \mathrm{sec}$

$\begin{array}{ll}\text { RG } & 201.48 \\ \text { DW } & 20.800\end{array}$

$\begin{array}{ll}\text { DW } & 20.800 \text { usec } \\ \text { DE } & 6.50 \text { usec }\end{array}$

TE $\quad 300.0 \mathrm{~K}$

D1 $200000000 \mathrm{sec}$
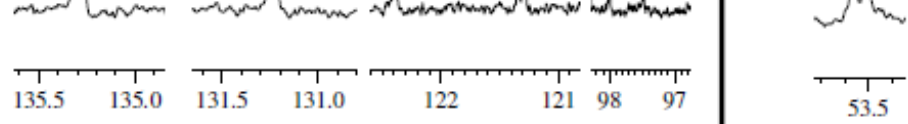

TD

$=\overline{\mathrm{SFO} 1}=\underset{100.6304993 \mathrm{MHz}}{\text { CHANNE }} \mathrm{f1}=$

NUC1 $13 \mathrm{C}$

$\begin{array}{ll}\text { P1 } & 9.90 \mathrm{usec} \\ \text { PLW1 } & 53.00000000 \mathrm{~W}\end{array}$

$=\bar{C}=$ CHANNEL $\mathrm{f}=$

$\mathrm{SFO} 2400.1621006 \mathrm{M}$

CPDPRG 2 waltz16

PCPD2
PLW2
$\begin{array}{ll}90.00 \text { usec } \\ 13.00000000 \mathrm{~W}\end{array}$

PLW12 $0.27963999 \mathrm{~W}$

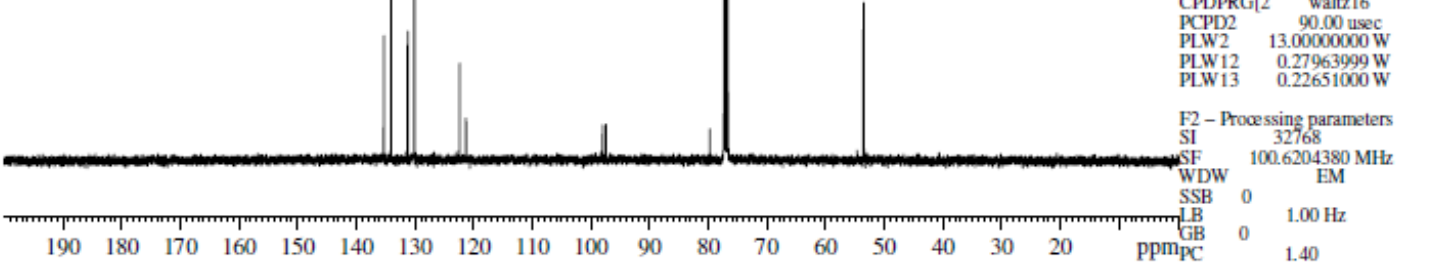

Figure 32: ${ }^{13} \mathrm{C}$ NMR spectrum of $4 \mathrm{~g}$ 


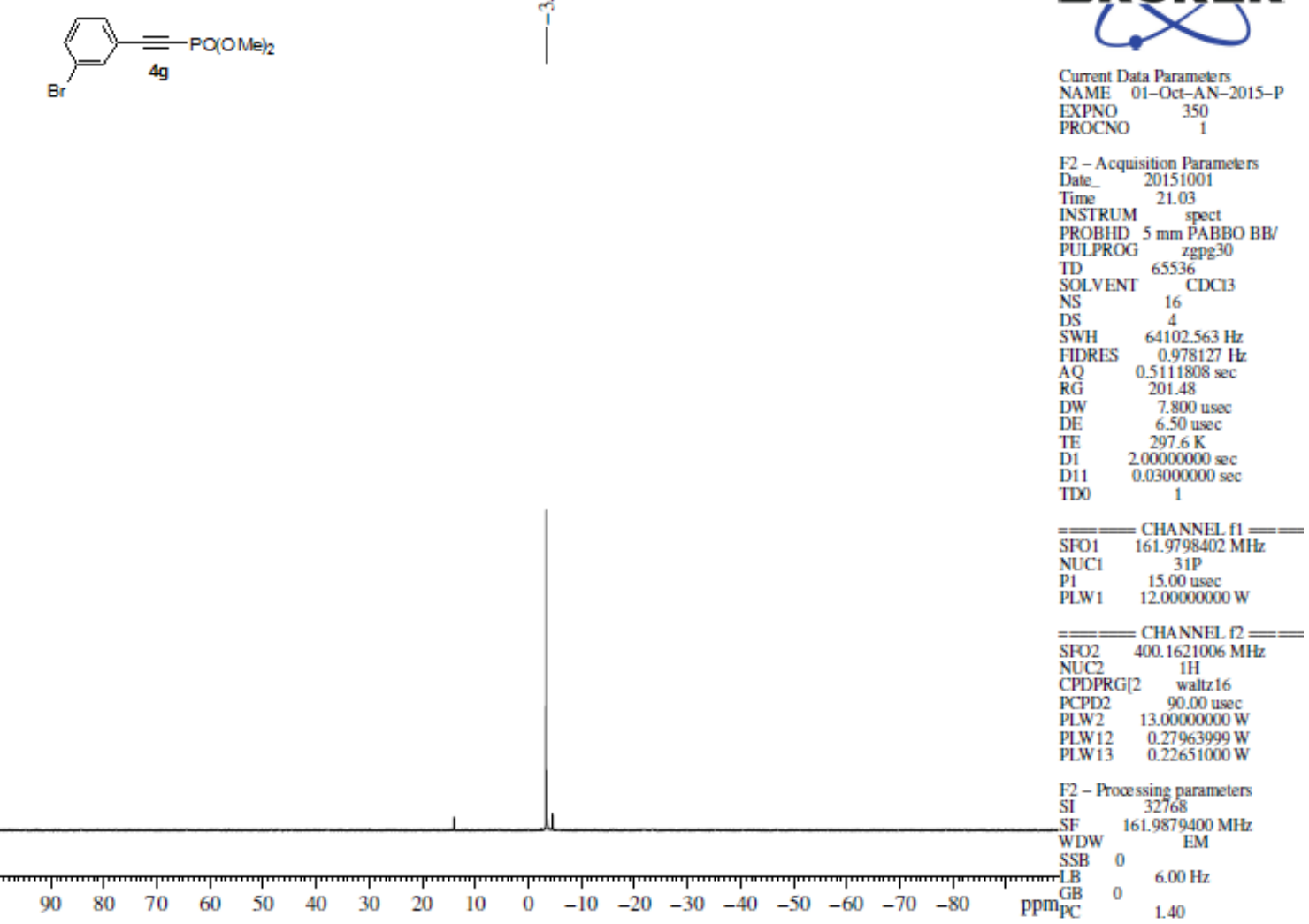

Figure 33: ${ }^{31} \mathrm{P}$ NMR spectrum of $4 \mathrm{~g}$

\section{NR BM-I-126}

BRUKER
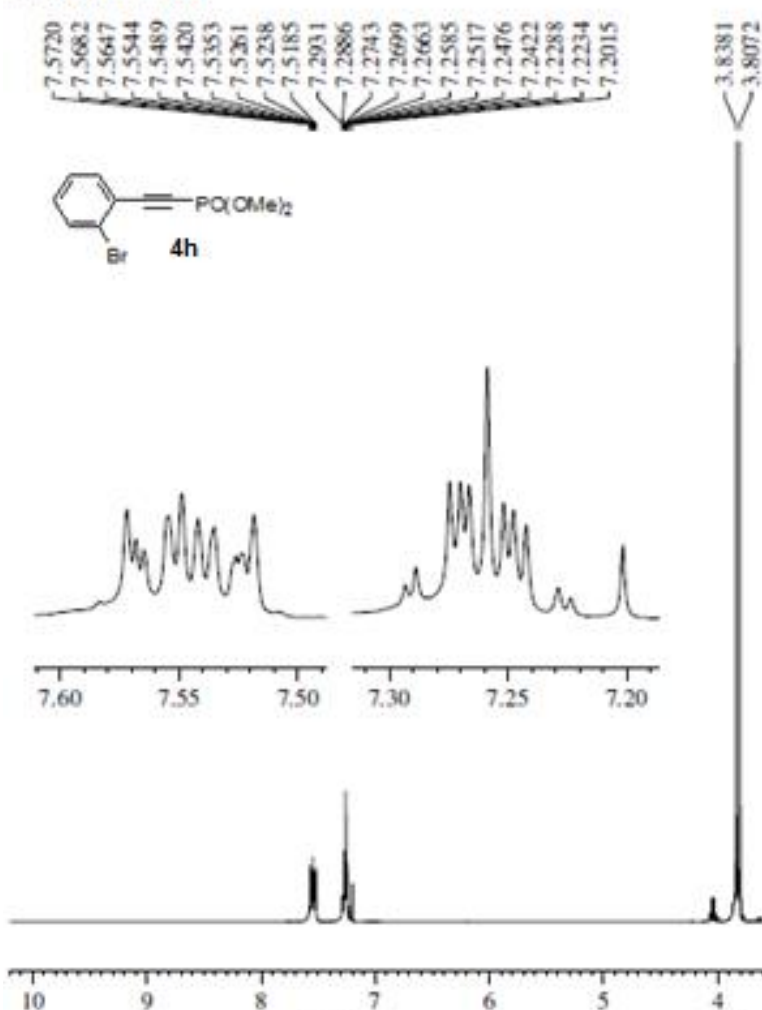

10

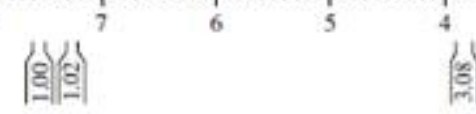

8

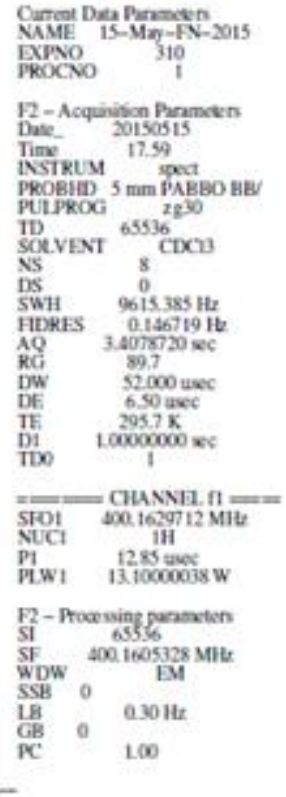

Figure 34: ${ }^{1} \mathrm{H}$ NMR spectrum of $4 \mathrm{~h}$ 


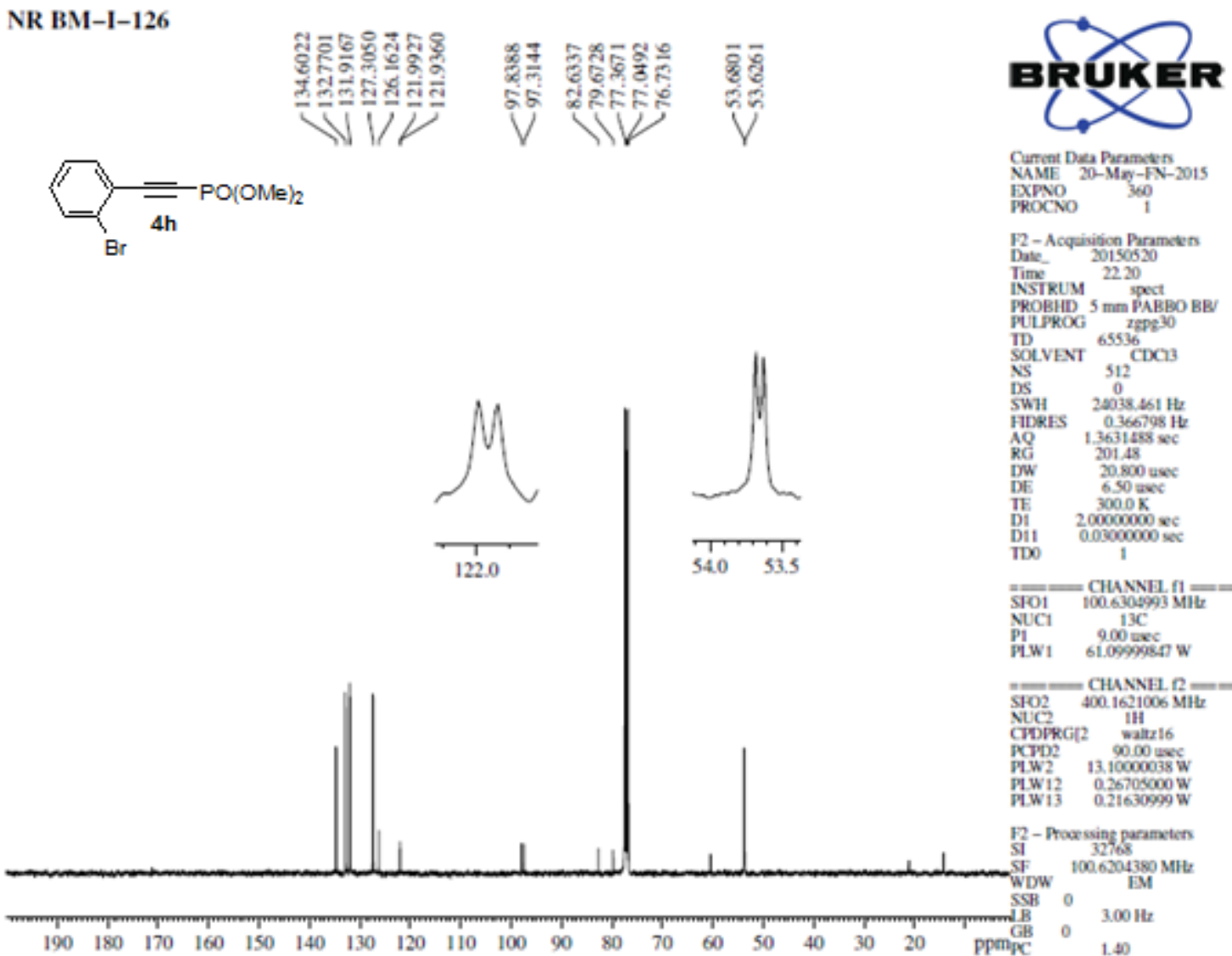

Figure 35: ${ }^{13} \mathrm{C}$ NMR spectrum of $4 \mathrm{~h}$

NR-BM-1-126

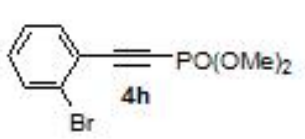

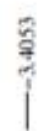

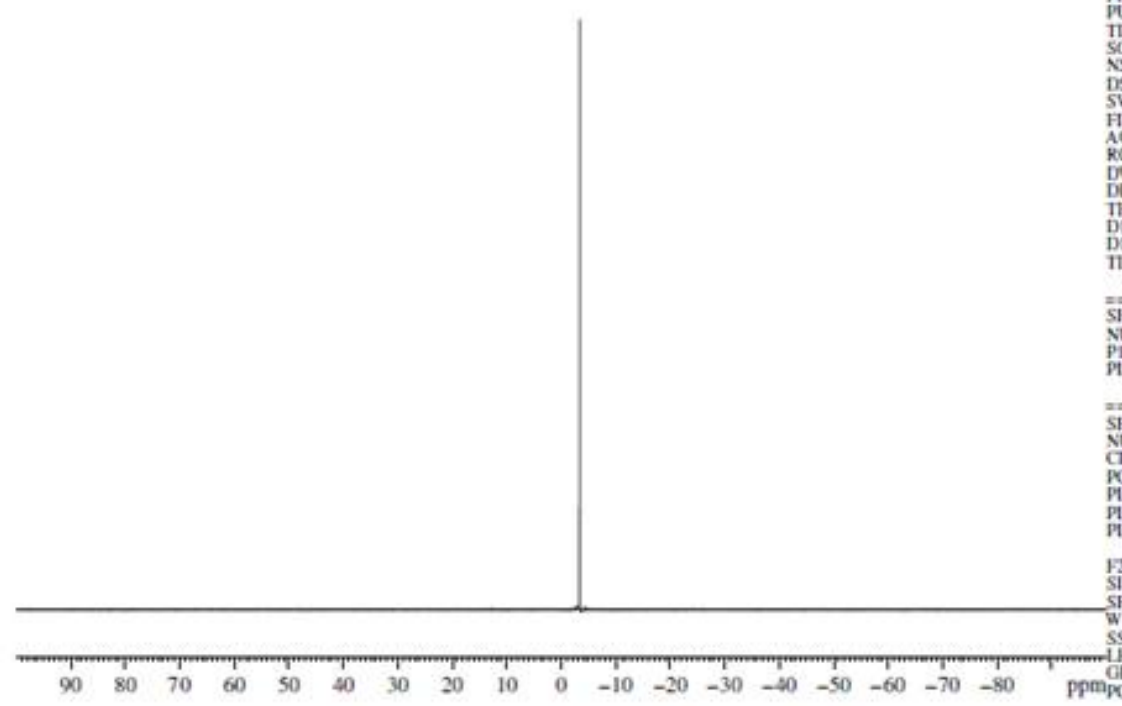

Figure 36: ${ }^{31} \mathrm{P}$ NMR spectrum of $4 \mathrm{~h}$

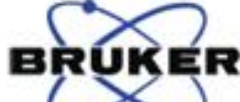

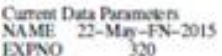
PROCD

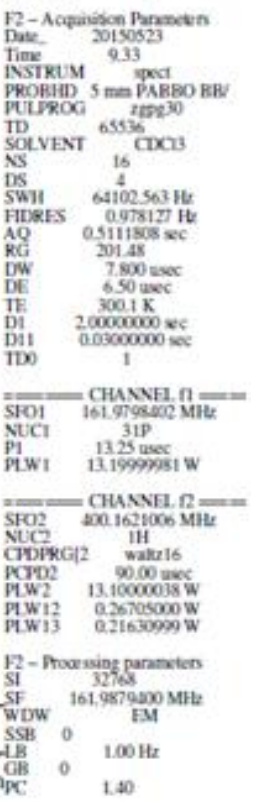

1.50 


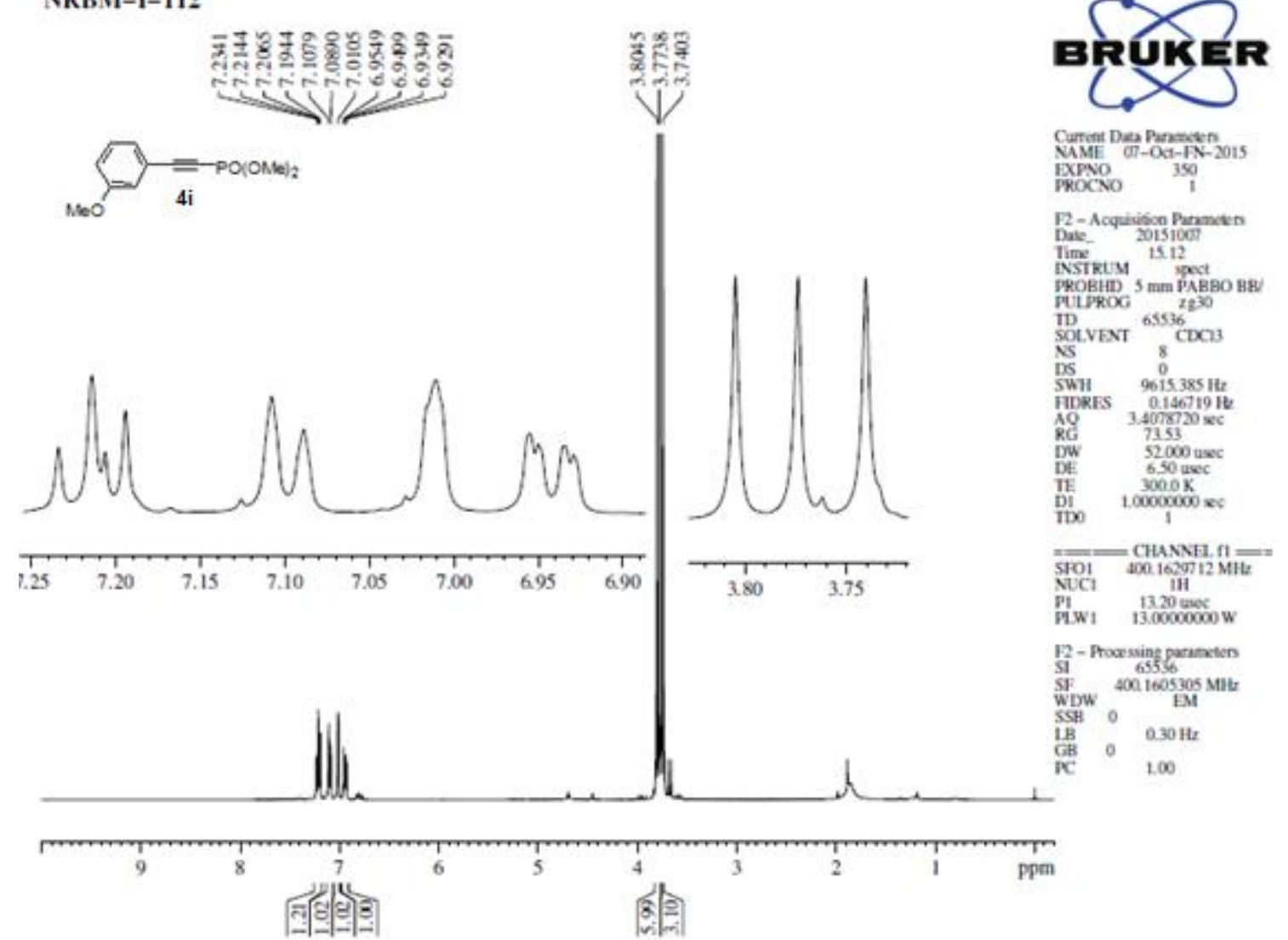

Figure 37: ${ }^{1} \mathrm{H}$ NMR spectrum of $4 \mathbf{i}$

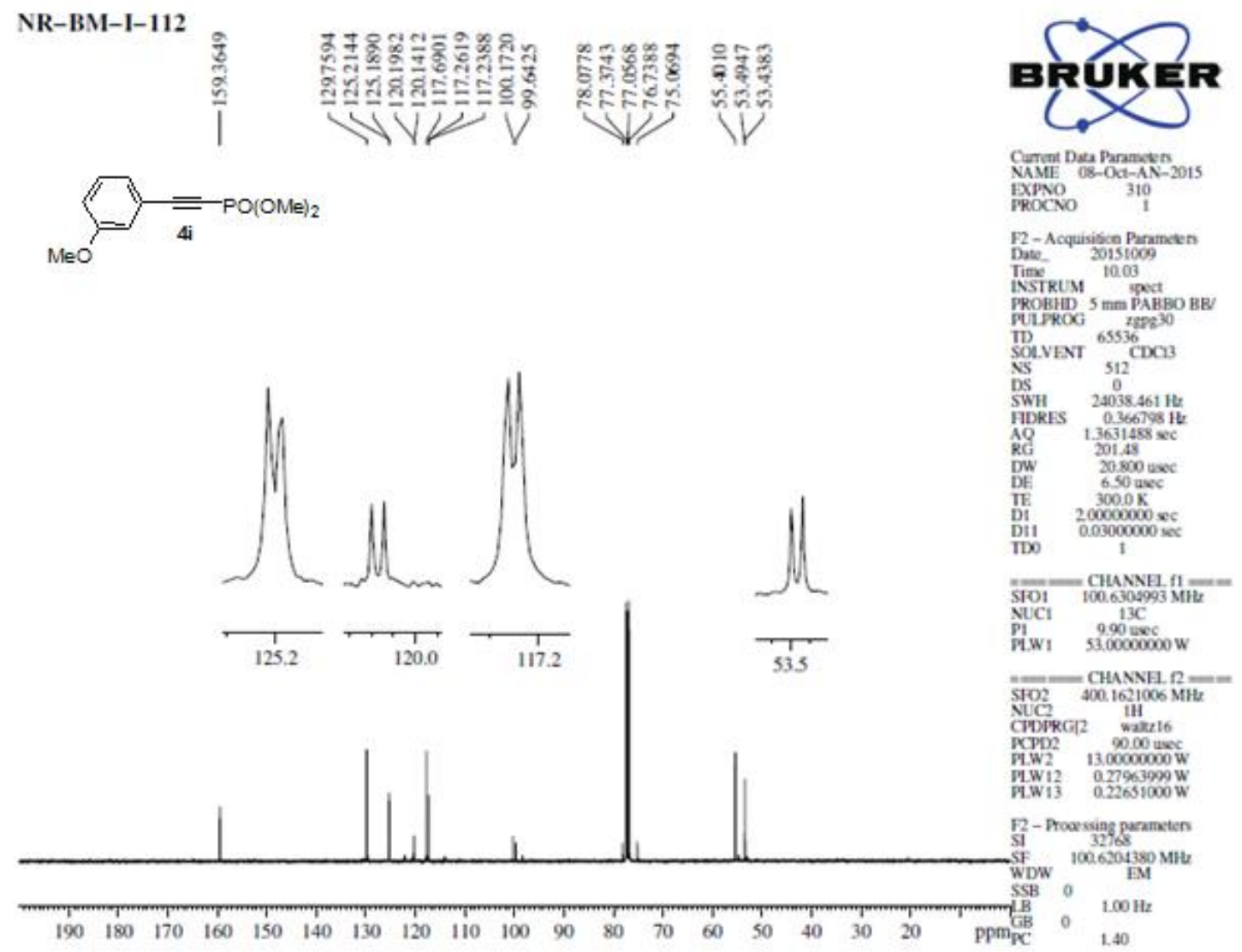

Figure 38: ${ }^{13} \mathrm{C}$ NMR spectrum of $4 i$ 


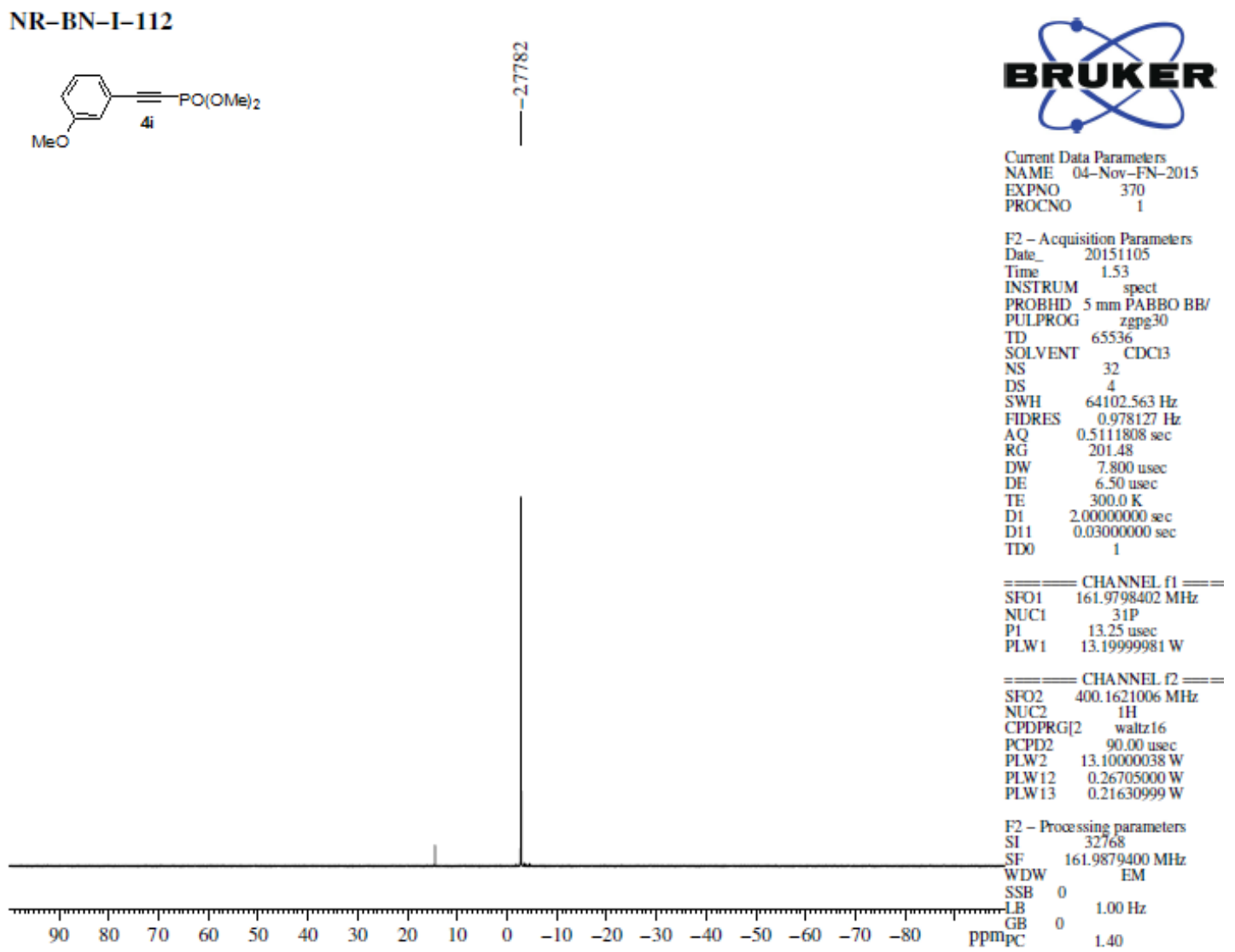

Figure 39: ${ }^{31} \mathrm{P}$ NMR spectrum of $4 \mathrm{i}$

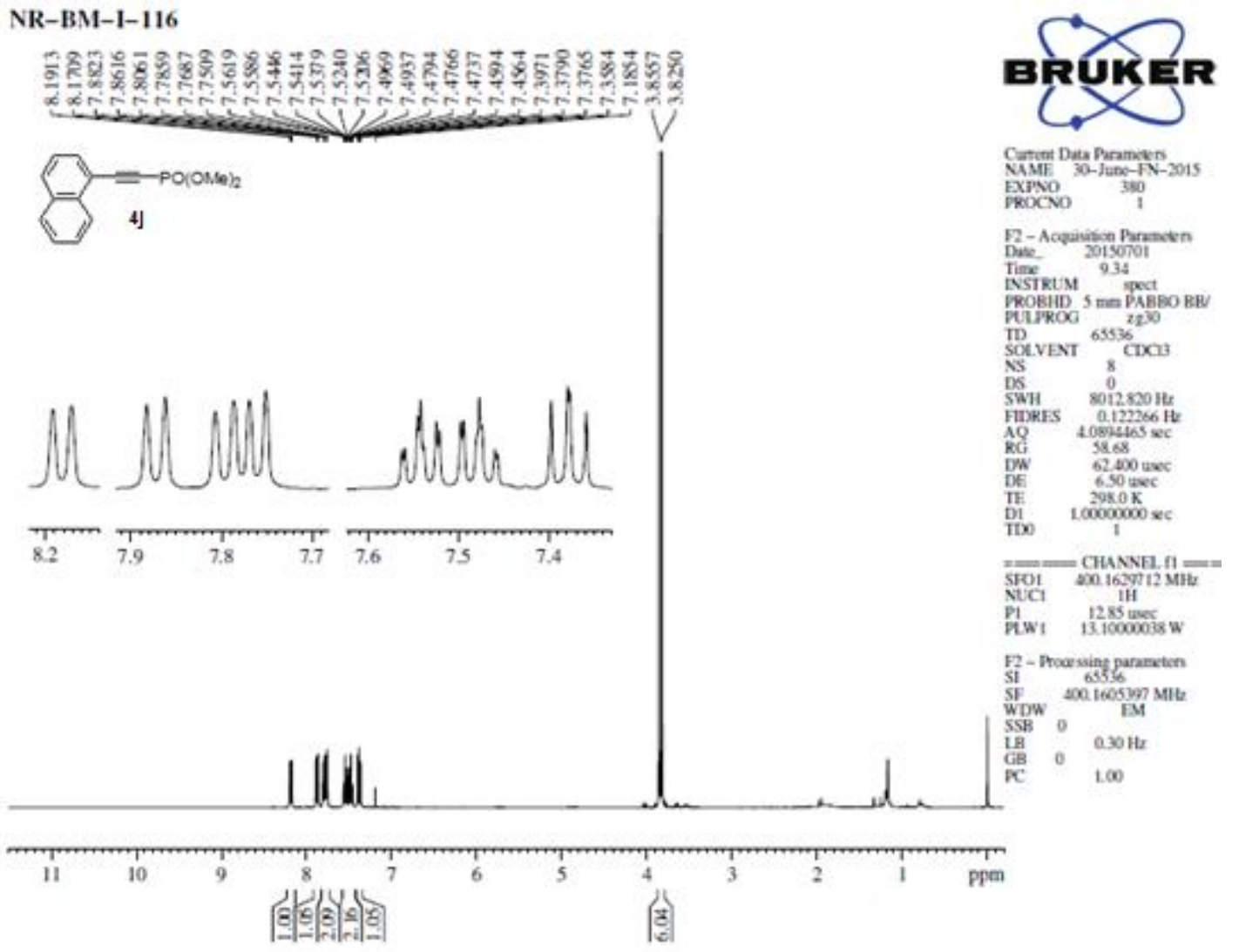

Figure 40: ${ }^{1} \mathrm{H}$ NMR spectrum of $4 \mathrm{j}$ 


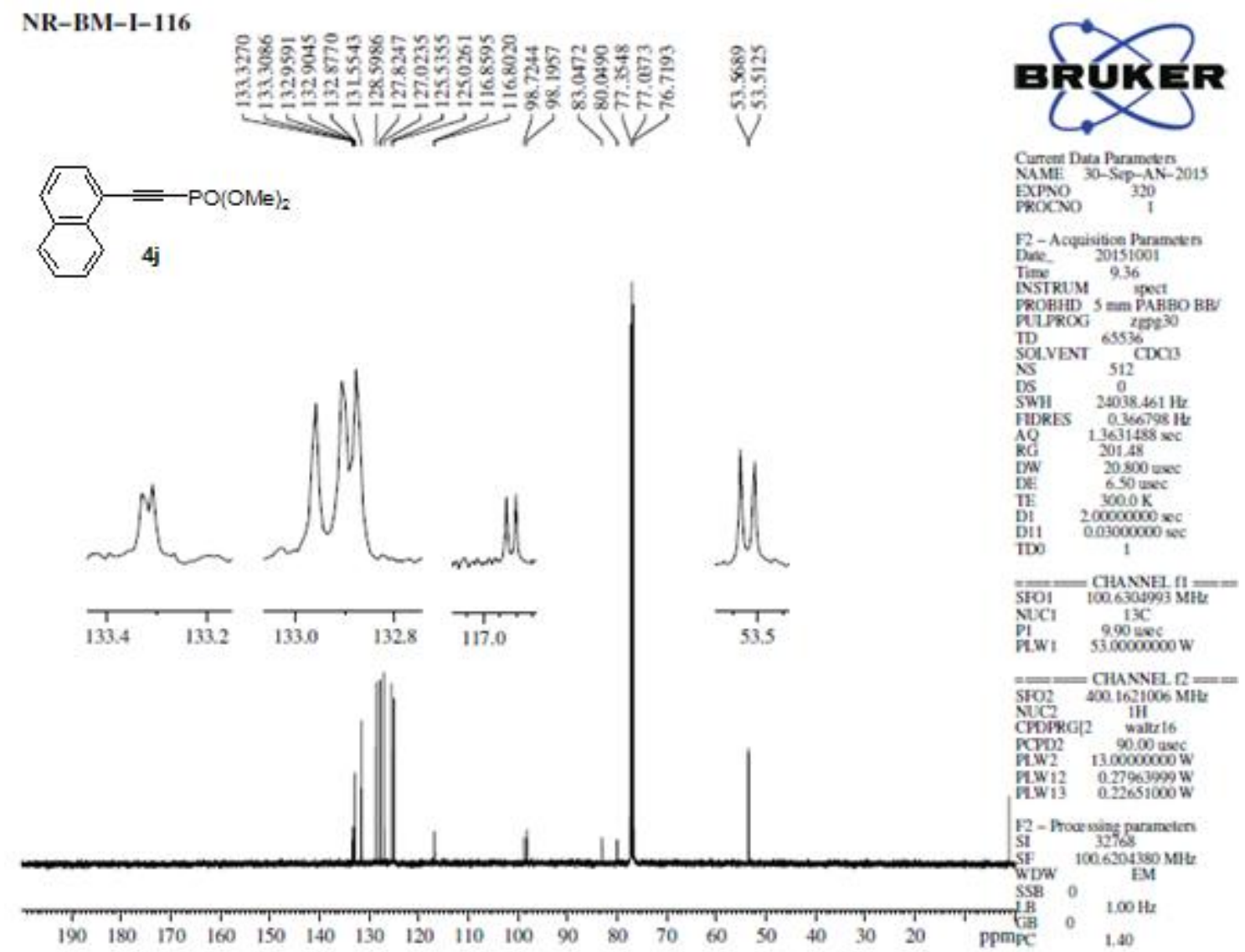

Figure $41:{ }^{13} \mathrm{C}$ NMR spectrum of $4 \mathrm{j}$

NR-BM-1-16
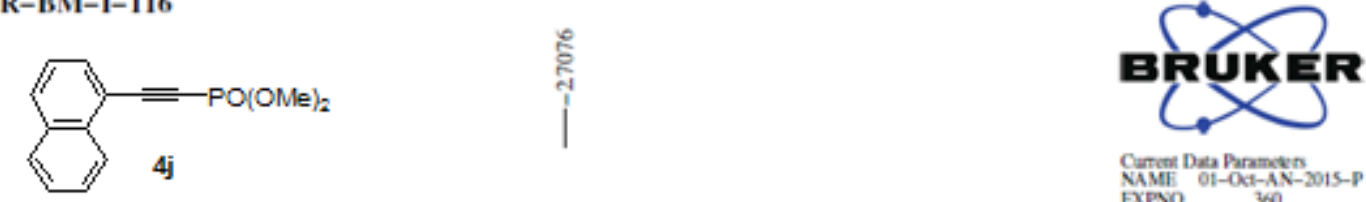

Cincen Daz Paramezs $\begin{array}{ll}\text { EXPNO } & 360 \\ \text { PROCNO } & 1\end{array}$

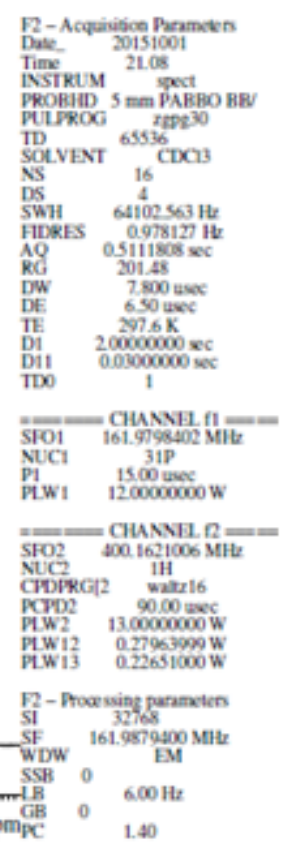

Figure 42: ${ }^{31} \mathrm{P}$ NMR spectrum of $4 \mathrm{j}$ 


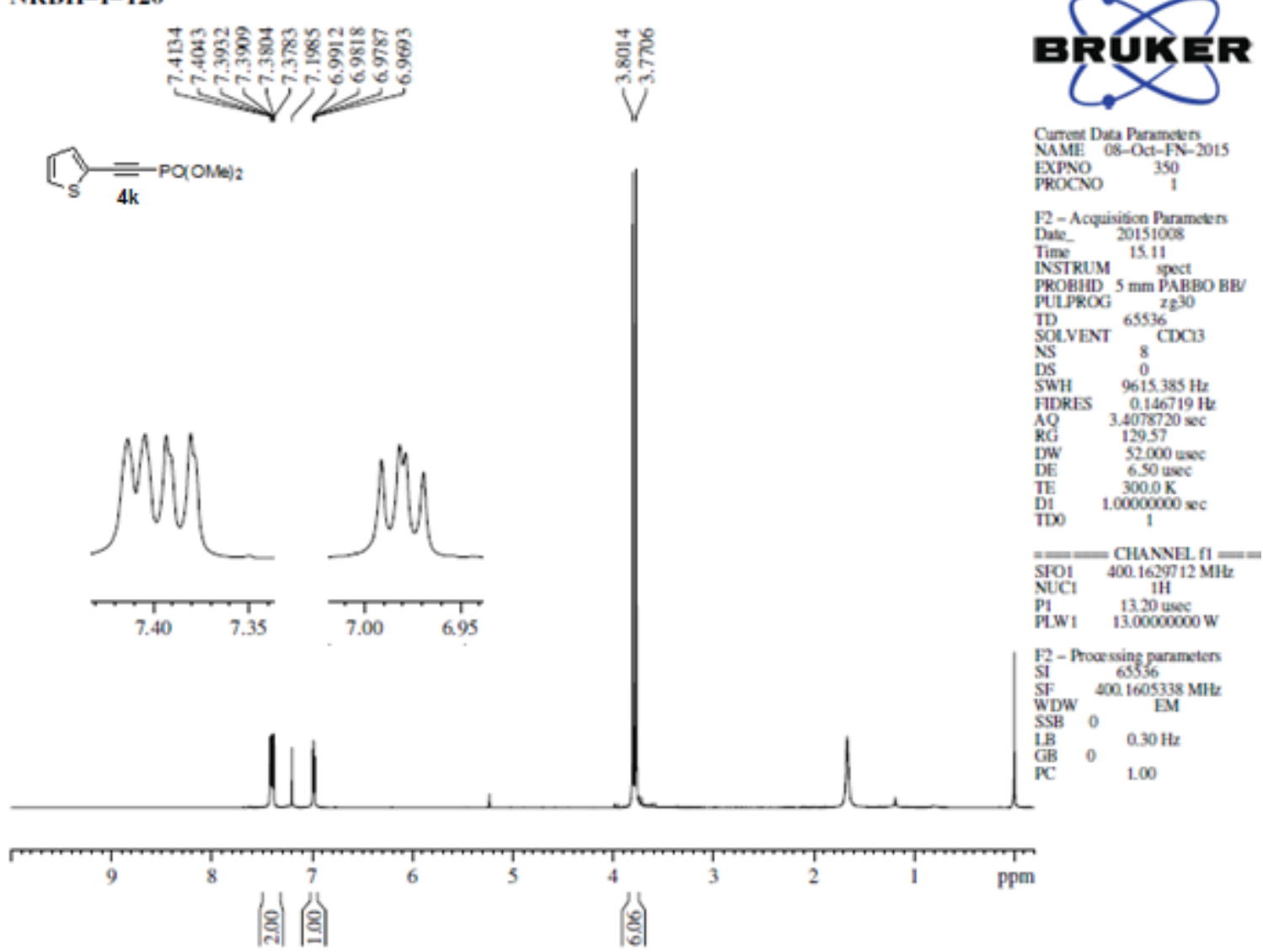

Figure 43: ${ }^{1} \mathrm{H}$ NMR spectrum of $4 k$
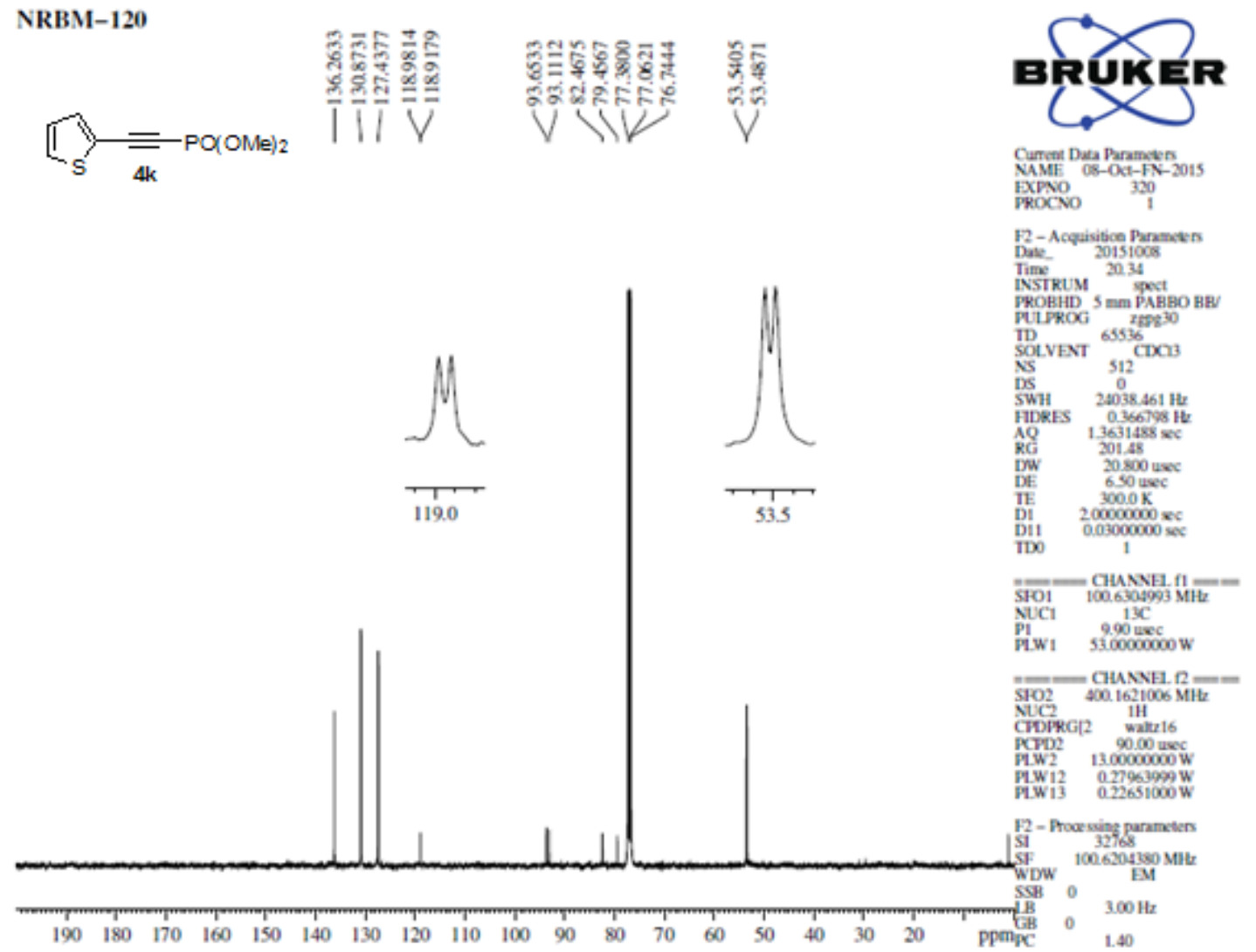

Figure 44: ${ }^{13} \mathrm{C}$ NMR spectrum of $4 \mathrm{k}$ 


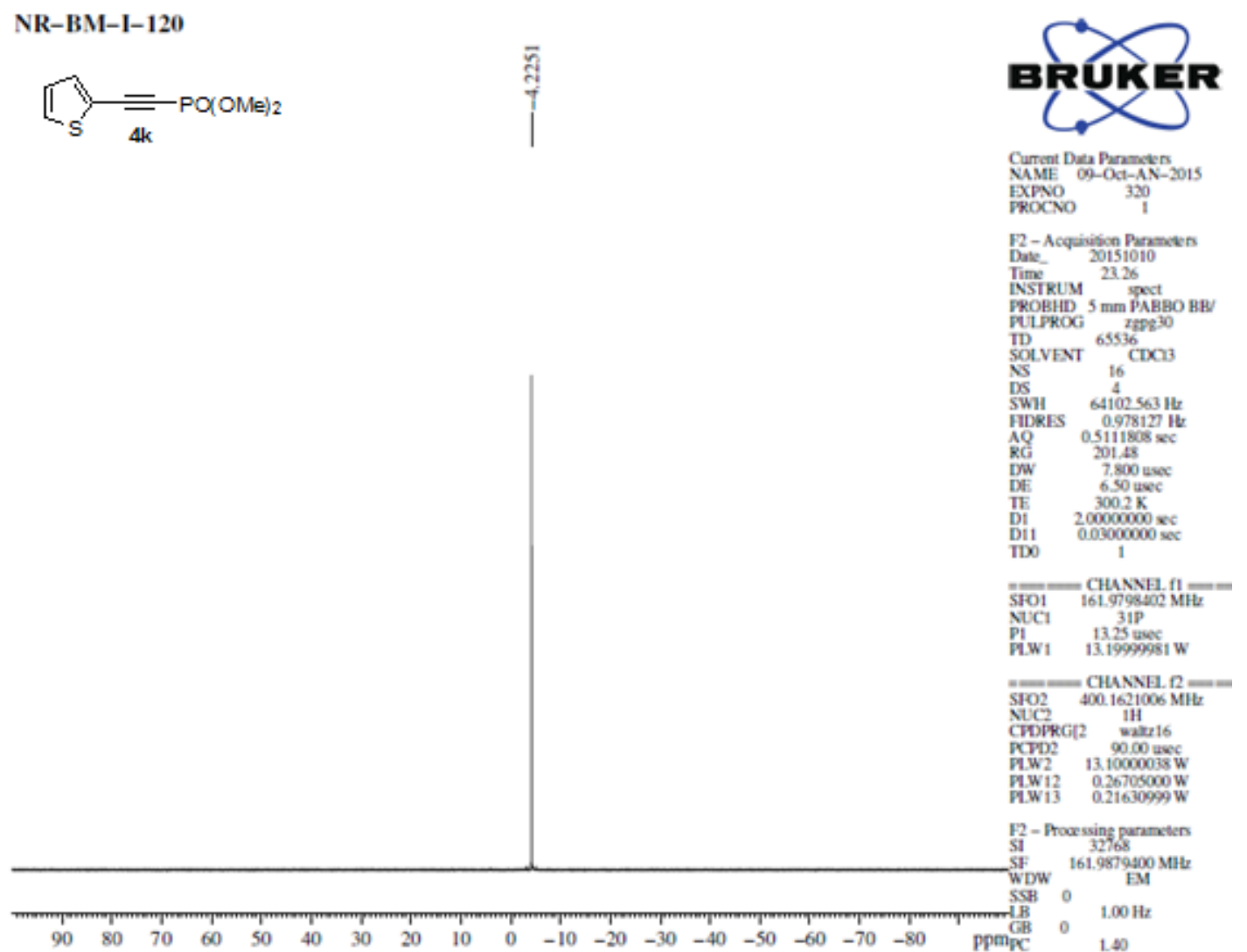

Figure 45: ${ }^{31} \mathrm{P}$ NMR spectrum of $4 k$

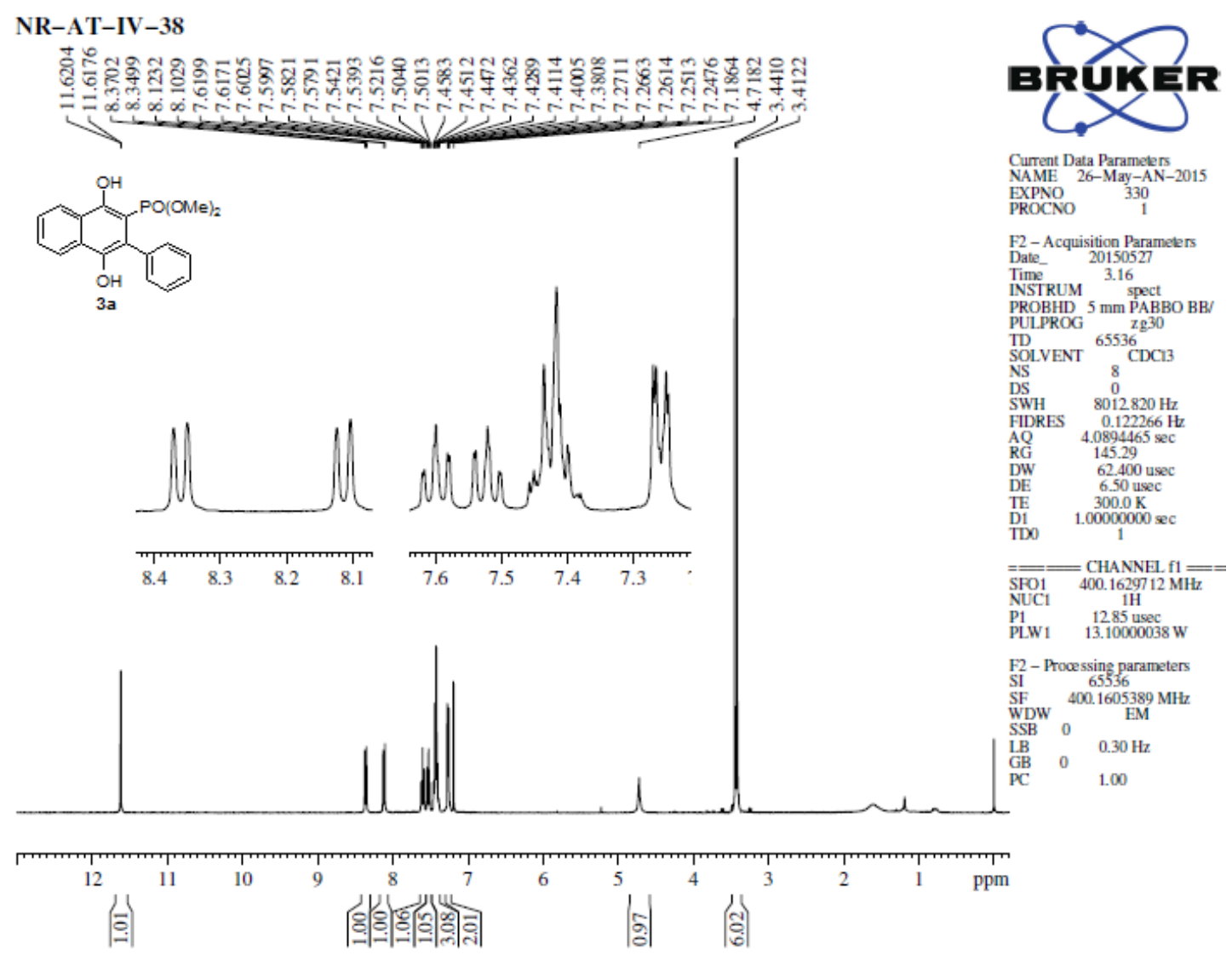

Figure 46: ${ }^{1} \mathrm{H}$ NMR spectrum of $3 a$ 


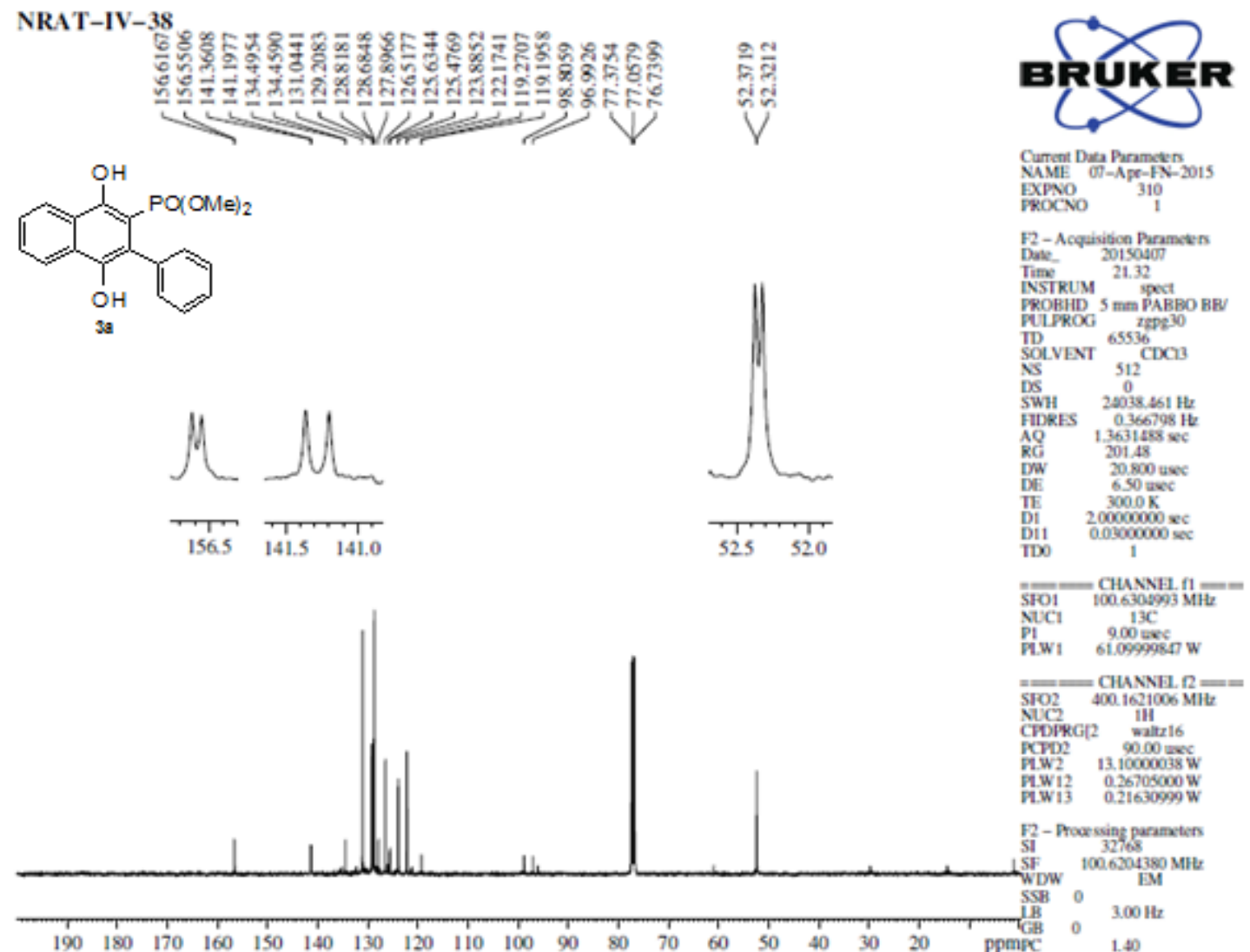

Figure 47: ${ }^{13} \mathrm{C}$ NMR spectrum of $3 a$

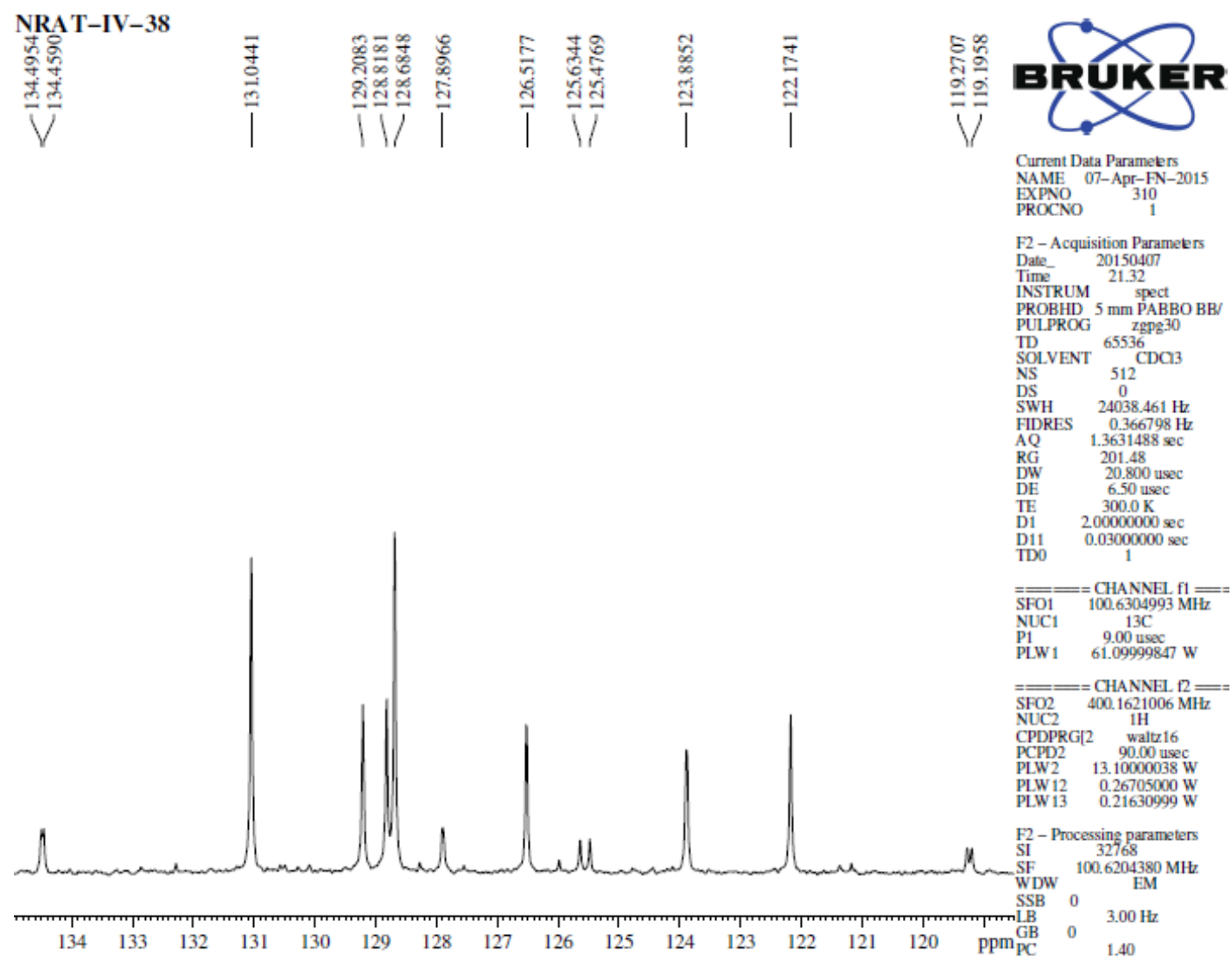

Figure 48: ${ }^{13} \mathrm{C}$ NMR spectrum of $3 a$ (expansion) 


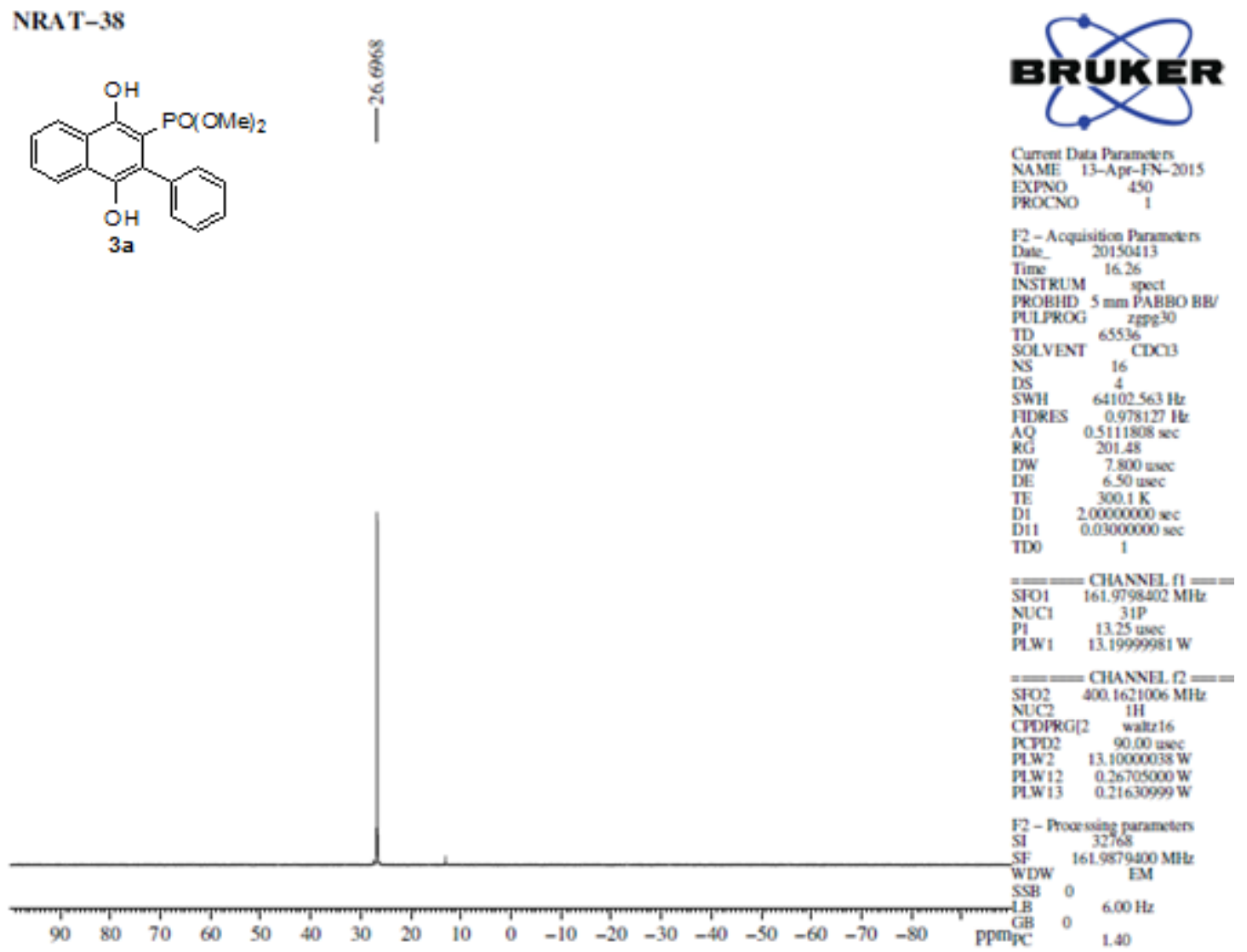

Figure 49: ${ }^{31} \mathrm{P}$ NMR spectrum of $3 a$

$$
\begin{aligned}
& \text { NRAT-IV-29 }
\end{aligned}
$$

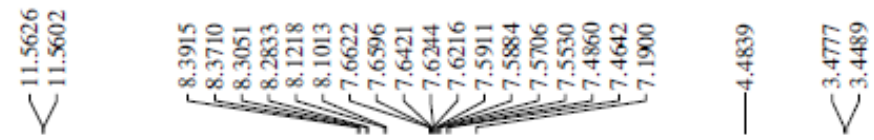<smiles>COc1c(-c2ccc([N+](=O)[O-])cc2)c(O)c2ccccc2c1O</smiles>
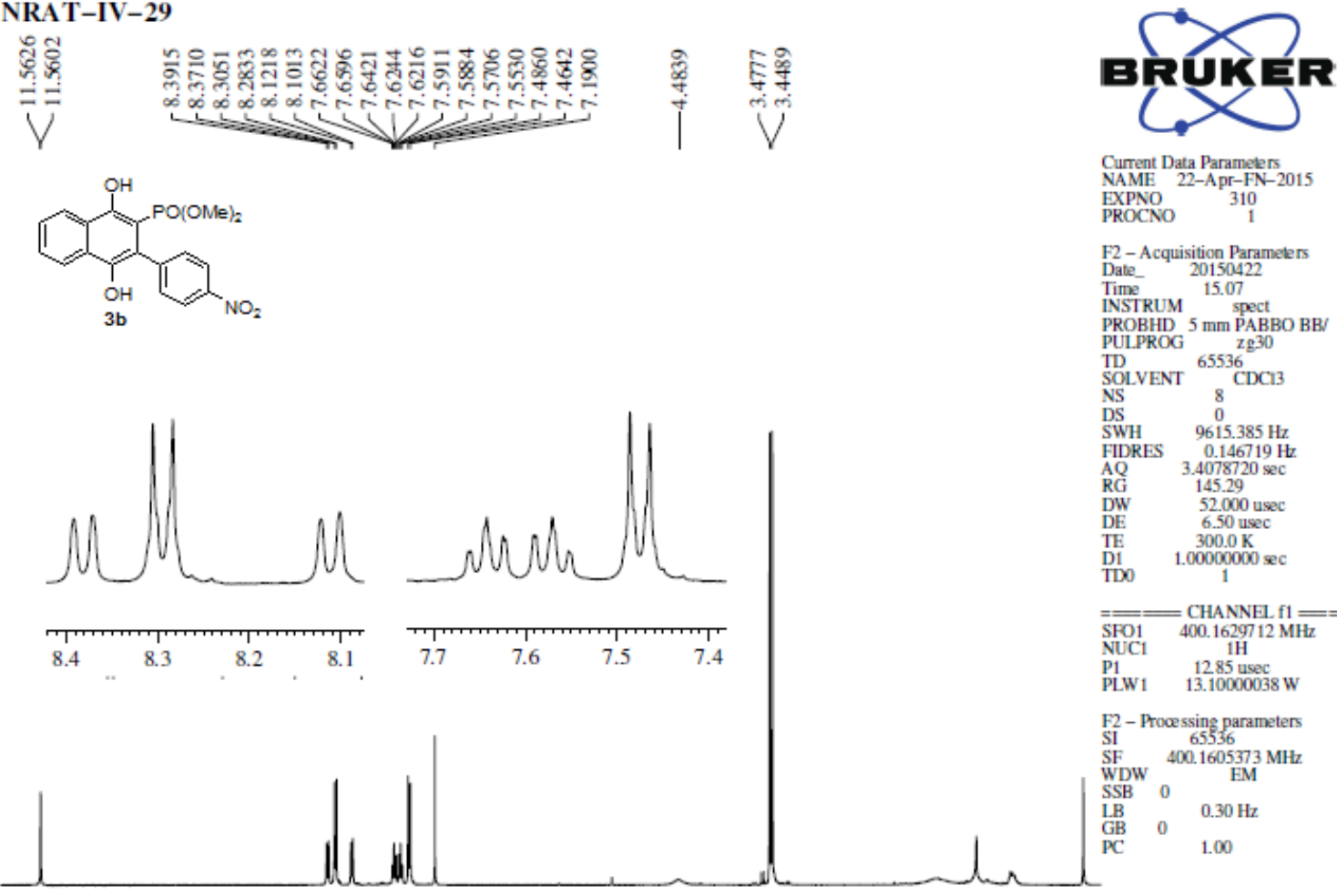

Current Data Parameters
NAME 22-A pr-FN-2015 $\begin{array}{lr}\text { EXPNO } & 310 \\ \text { PROCNO } & 1\end{array}$

F2 - Acquisition Parameters Time_ 15.07 INSTRUM spect PROBPRD 5 mm PABBO BB TD 65536 NS $\begin{array}{lcl}\text { NS } & 8 \\ \text { DS } & 0 \\ \text { SWH } & 9615385 \mathrm{H}\end{array}$ FIDRES $\quad 0.146719$ $\mathrm{AQ} \quad 3.4078720 \mathrm{sec}$

DW $\quad 52.000$ usec TE $300.0 \mathrm{~K}$ 1.00000000 $=\overline{\overline{S F 1}}=$ CHANNEL $\mathrm{f1}=$ 1H $\begin{array}{ll}\text { P1 } & 12.85 \text { usec } \\ \text { PLW1 } & 13.10000038 \mathrm{~W}\end{array}$ F2 - Processing parameters $\begin{array}{ll}\text { SI } & 65536 \\ \text { SF } & 400.1605373 \mathrm{MHz}\end{array}$ SSB 0 EM GB $\quad 0.30 \mathrm{~Hz}$

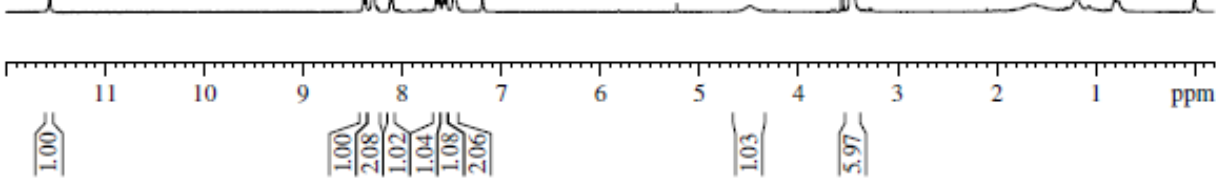

Figure 50: ${ }^{1} \mathrm{H}$ NMR spectrum of $3 \mathrm{~b}$ 


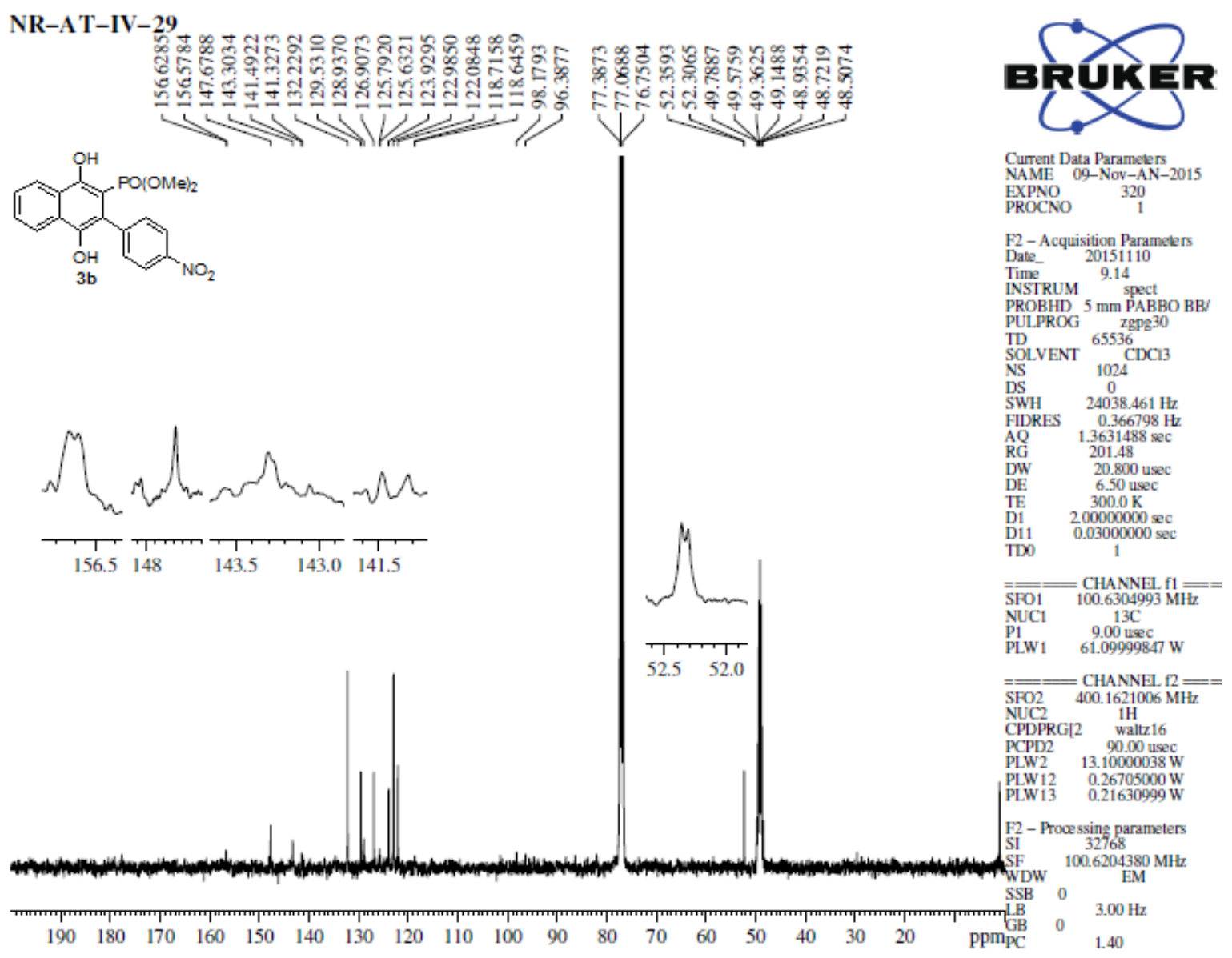

Figure 51: ${ }^{13} \mathrm{C}$ NMR spectrum of $3 \mathrm{~b}$

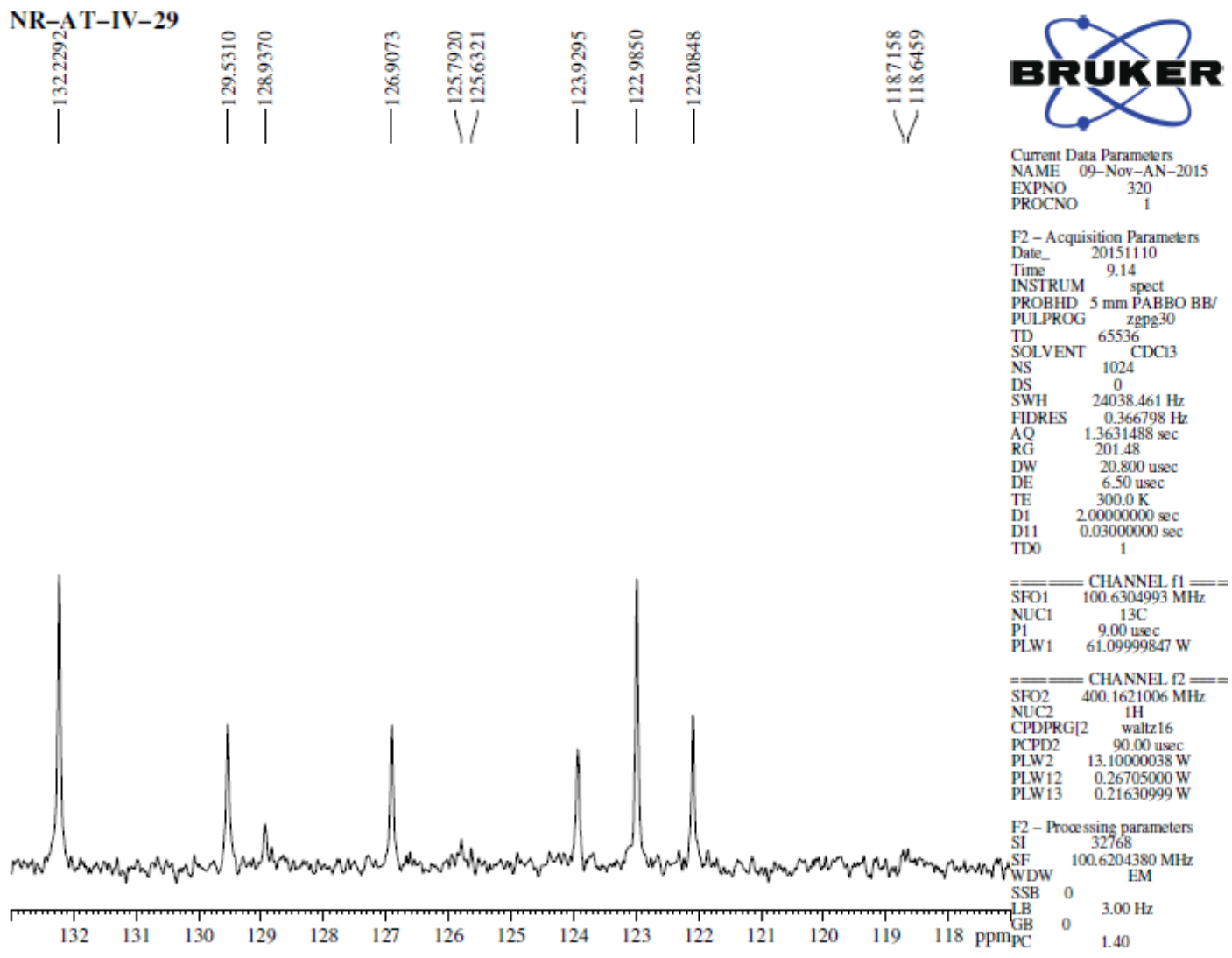

Figure 52: ${ }^{13} \mathrm{C}$ NMR spectrum of $3 b$ (expansion) 
NRAT-IV-29
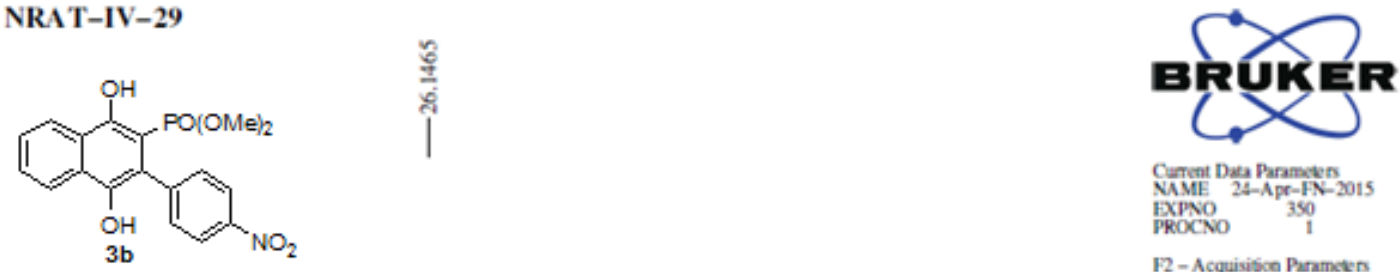

Yunger Das Parameses

NXPNO $25-\mathrm{APC}-\mathrm{NO}$

F2-Acquiviscon Prametes
Date 20150425

Tive 1351

INSTRLM 1 M Pet

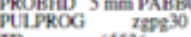

SOAVENT ${ }^{65536} \mathrm{CDC}$

NS 16

SWH

HDRES $64102563 \mathrm{Ht}$

AORES 0.597111808 sec

$\begin{array}{ll}\text { DW } & 7.500 \text { usec } \\ \text { DE } & 650 \text { usec } \\ \text { DE } & 3002\end{array}$

IE $30002 \mathrm{~K}$

Di1 $0.03000000 \mathrm{sec}$

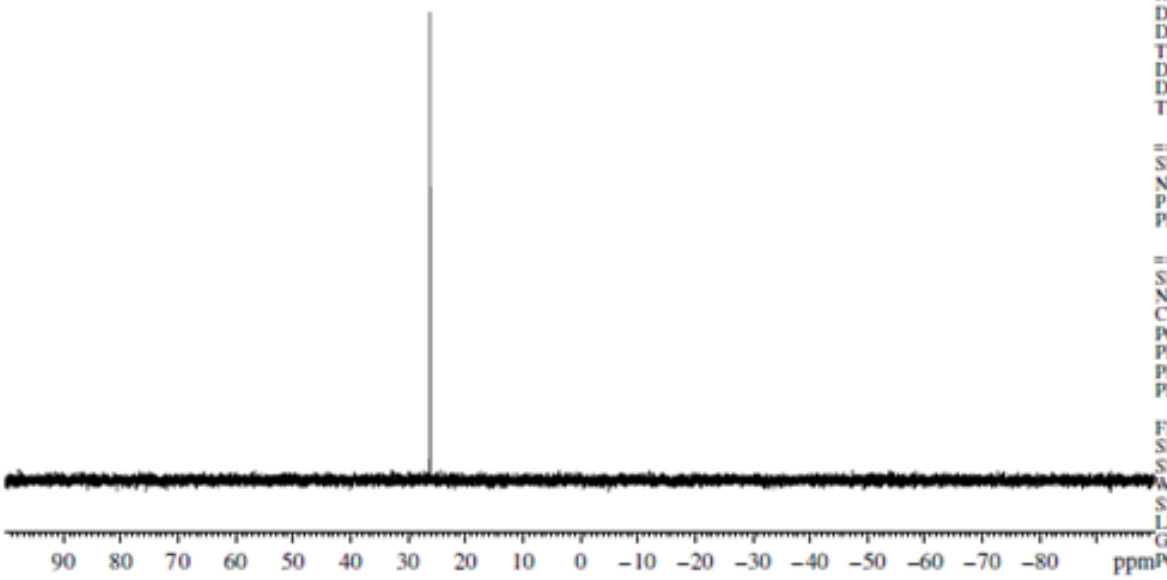

sFo1 $={ }_{161.97985002} \mathrm{M}=$

NuC1 319

PIW1 13.25 awee

SFO2 $={ }_{400.1621006 \mathrm{MH}}^{\text {CHANEL }}$

NUC2 it

PCPD2 90.00 usec

PLW13 $0.21630999 \mathrm{~W}$

F2- Proorsing purameten $161.9879400 \mathrm{MH}$

DM

Figure 53: ${ }^{31} \mathrm{P}$ NMR spectrum of $3 \mathrm{~b}$

NRAT-IV-58

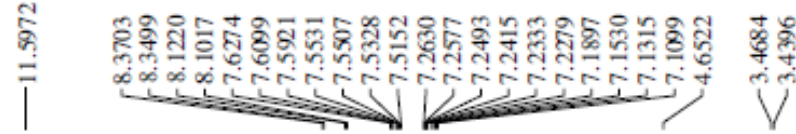

1.001

rigre 53: 31 NMR spectrum of 3 b

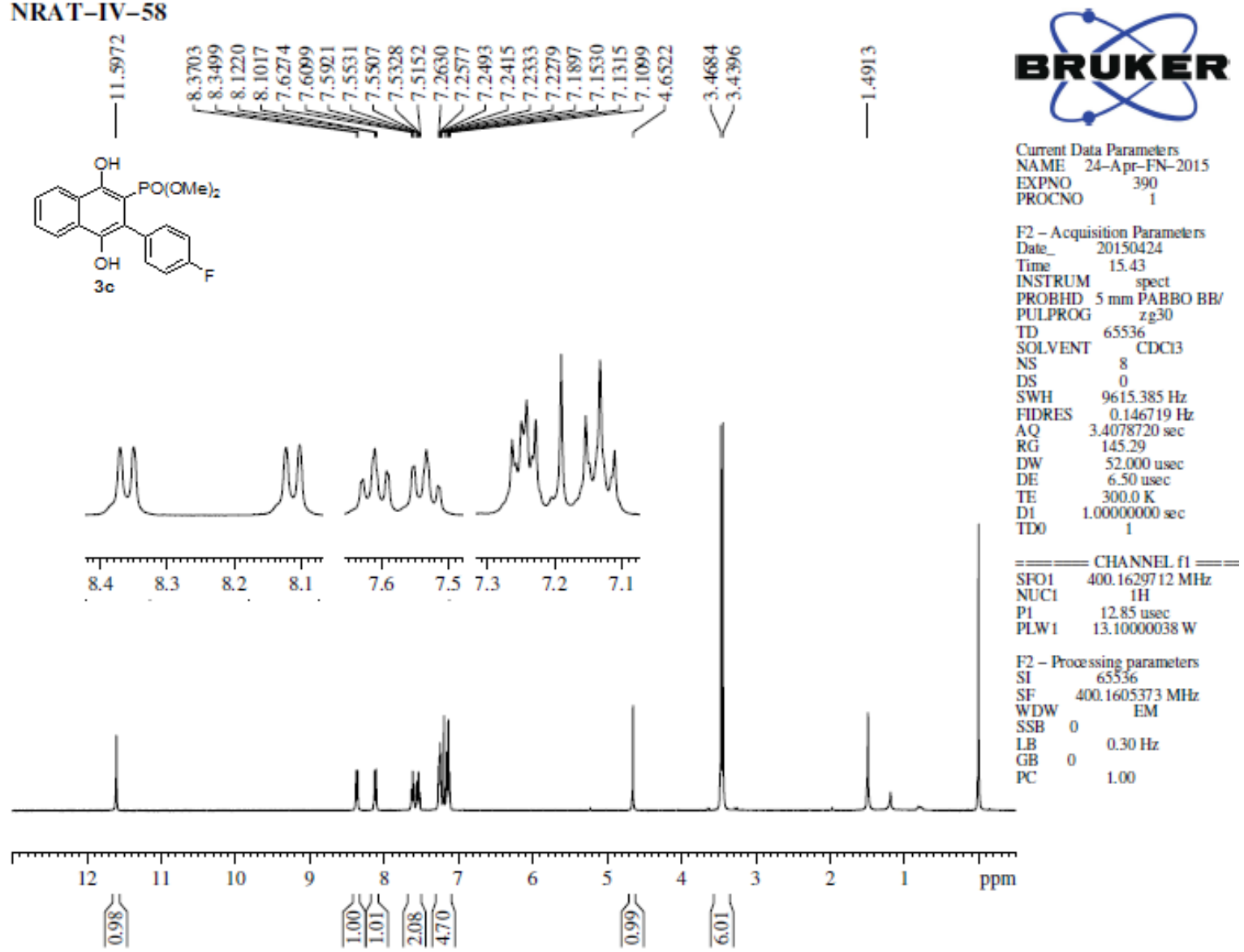

Figure 54: ${ }^{1} \mathrm{H}$ NMR spectrum of $3 \mathrm{c}$ 


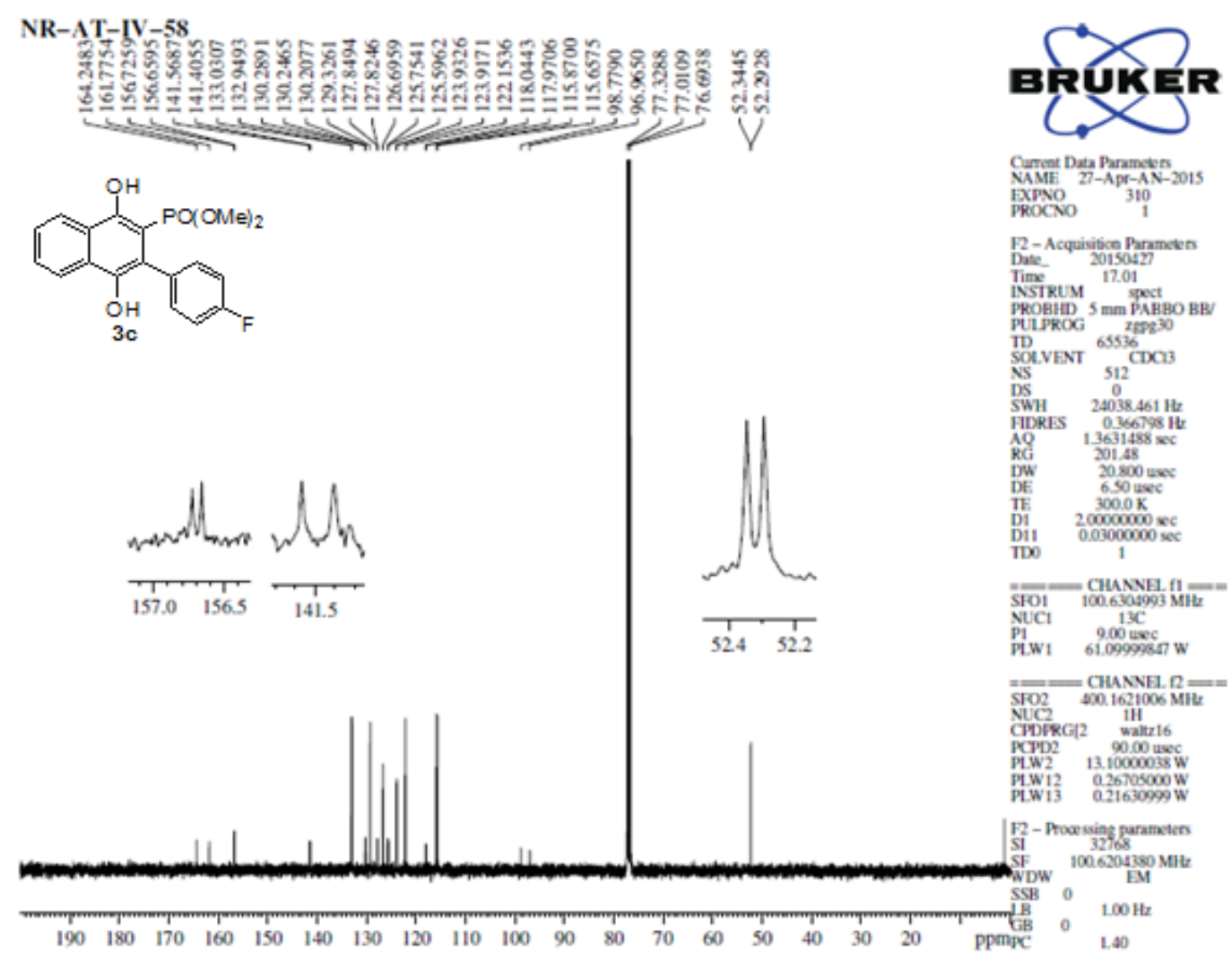

Figure 55: ${ }^{13} \mathrm{C}$ NMR spectrum of $3 \mathrm{c}$
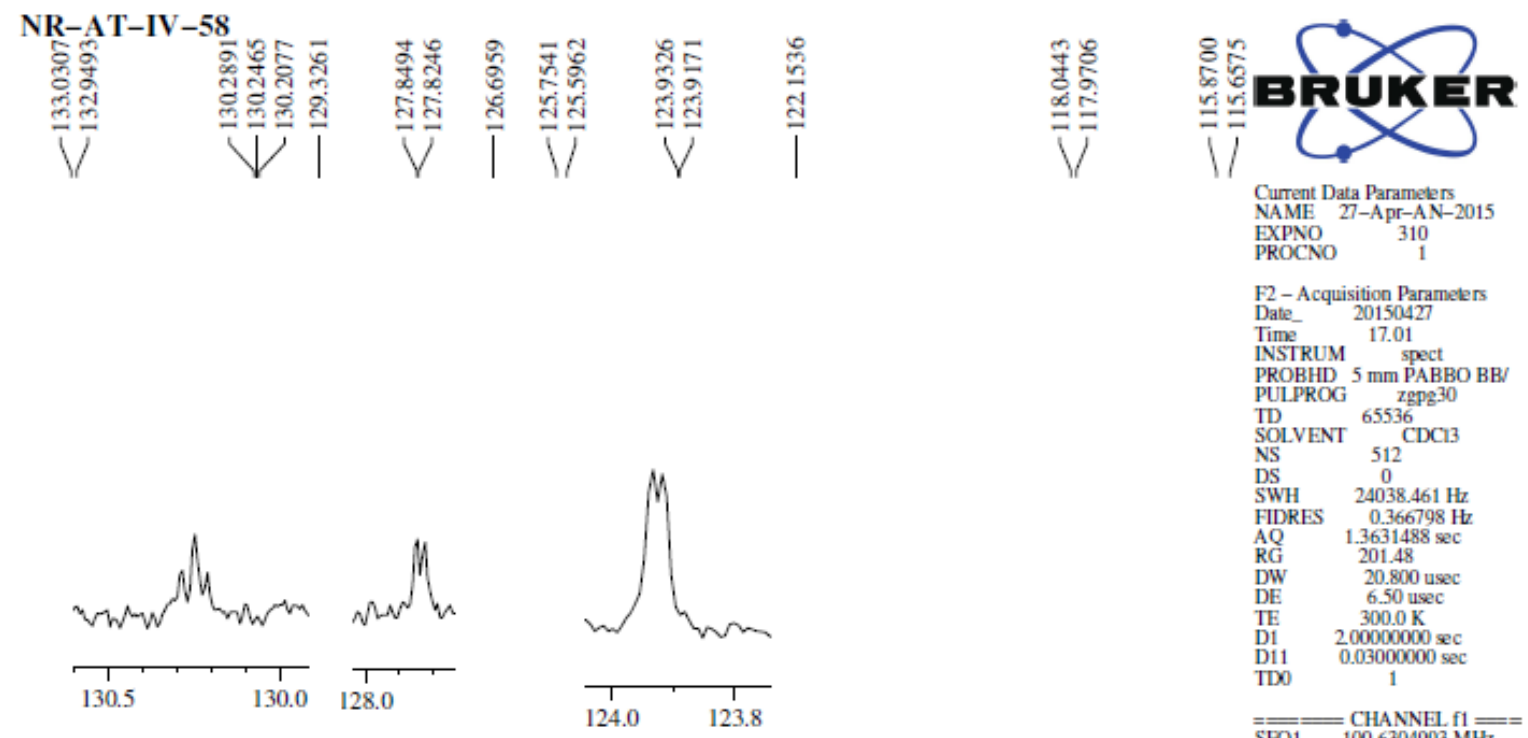

Current Data Parameters
NAME $27-A p r-A N-2015$ $\begin{array}{lr}\text { EXPNO } & 310 \\ \text { PROCNO } & 1\end{array}$

F2 - Acquisition Parameters

Date_ 20150427

Time 17.01

NSTRUM spect ${ }_{\text {PULPROG }}^{\text {PROPB:30 }}$ TD $_{\text {SOLVENT }}{ }^{65536} \mathrm{CDC13}$ SOLVENT ${ }_{512} \mathrm{CDC} 3$ $\begin{array}{lc}\text { NS } & 512 \\ \text { DS } & 0 \\ \text { SWH } & 24038.461 \mathrm{~Hz} \\ \text { FIDRES } & 0.366798 \mathrm{~Hz}\end{array}$ AQ $1.3631488 \mathrm{sec}$ RG 201.48

$\begin{array}{ll}\text { DW } & 20.800 \text { usec } \\ \text { DE } & 6.50 \text { usec }\end{array}$

TE $\quad 300.0 \mathrm{~K}$

$\begin{array}{ll}\text { D1 } & 200000000 \mathrm{sec} \\ \text { D11 } & 0.03000000 \mathrm{sec}\end{array}$

$\begin{array}{lll}130.5 & 130.0 & 128.0\end{array}$

TD

$=\overline{\text { SFO1 }}=\underset{100.6304993 \mathrm{MHz}}{\text { CHANNE }} \mathrm{f1}=$

NUC1 $\quad 13 \mathrm{C}$

$\begin{array}{ll}\text { P1 } & 9.00 \text { usec } \\ \text { PLW1 } & 61.09999847 \mathrm{~W}\end{array}$

$\overline{\overline{\mathrm{FO} 2}}=$ CHANNEL $\mathrm{f} 2=$

$\mathrm{SFO} 2 \quad 400.1621006 \mathrm{MH}$

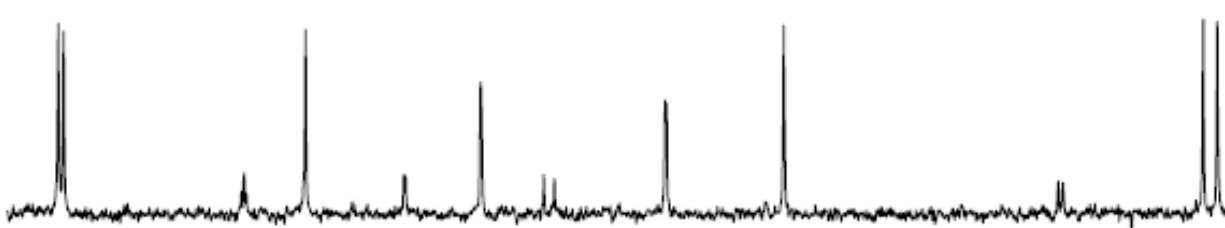

CPDPRE2 $1 \mathrm{H}$

$\begin{array}{lr}\text { PCPD2 } & 90.00 \text { usec } \\ \text { PLW2 } & 13.10000038 \mathrm{~W}\end{array}$

$\begin{array}{ll}\text { PLW13 } & 0.26705000 \mathrm{~W}\end{array}$

F2 - Procssing parameters

$\begin{array}{ll}\text { SI } & 32768 \\ \text { SF } & 100.6204380 \mathrm{MHz}\end{array}$

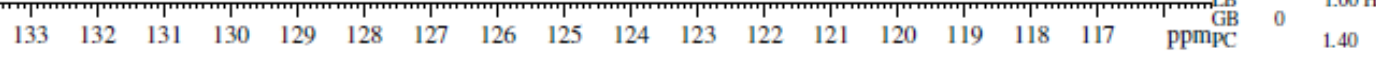

Figure 56: ${ }^{13} \mathrm{C}$ NMR spectrum of $3 \mathrm{c}$ (expansion) 


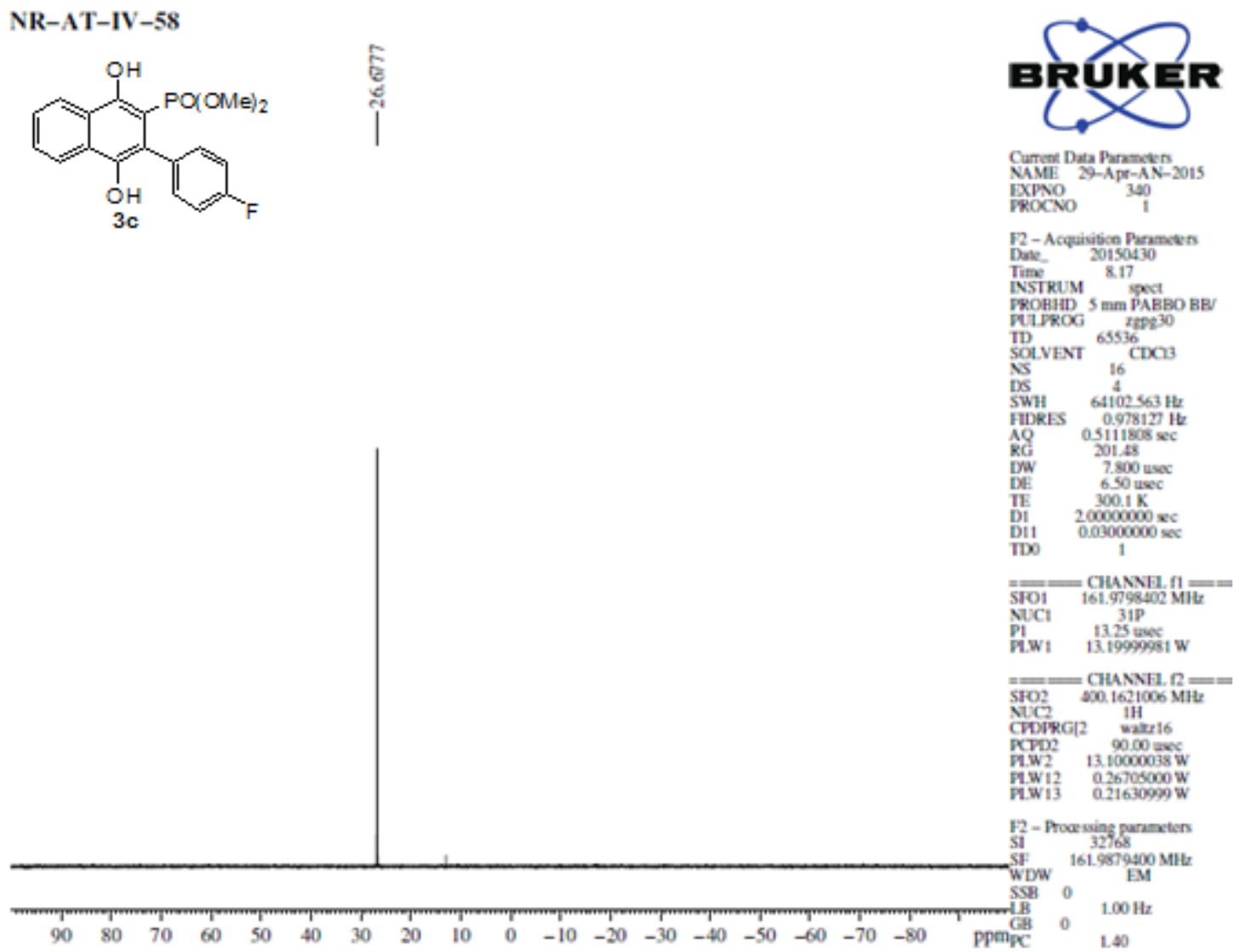

Figure 57: ${ }^{31} \mathrm{P}$ NMR spectrum of $3 \mathrm{c}$

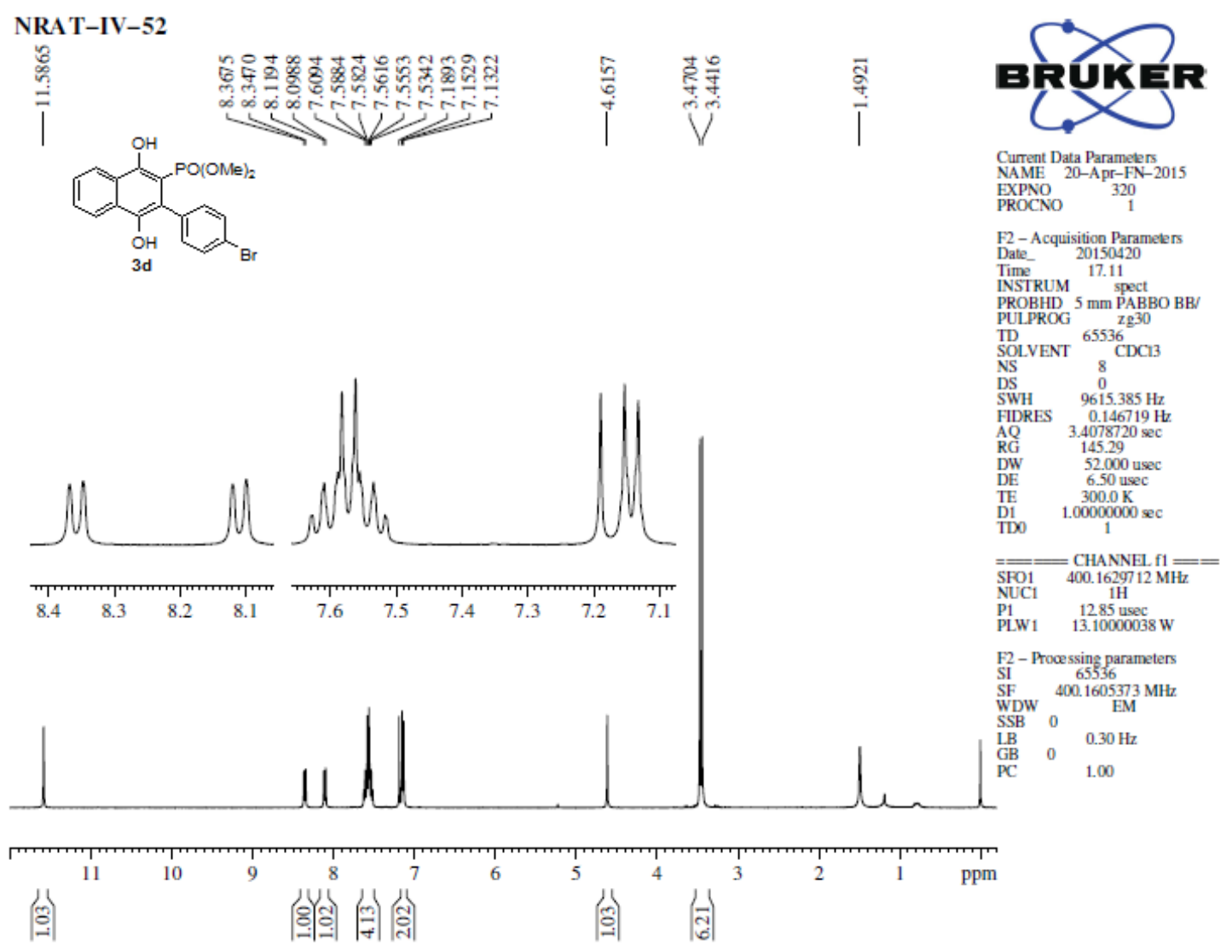

Figure 58: ${ }^{1} \mathrm{H}$ NMR spectrum of $3 d$ 


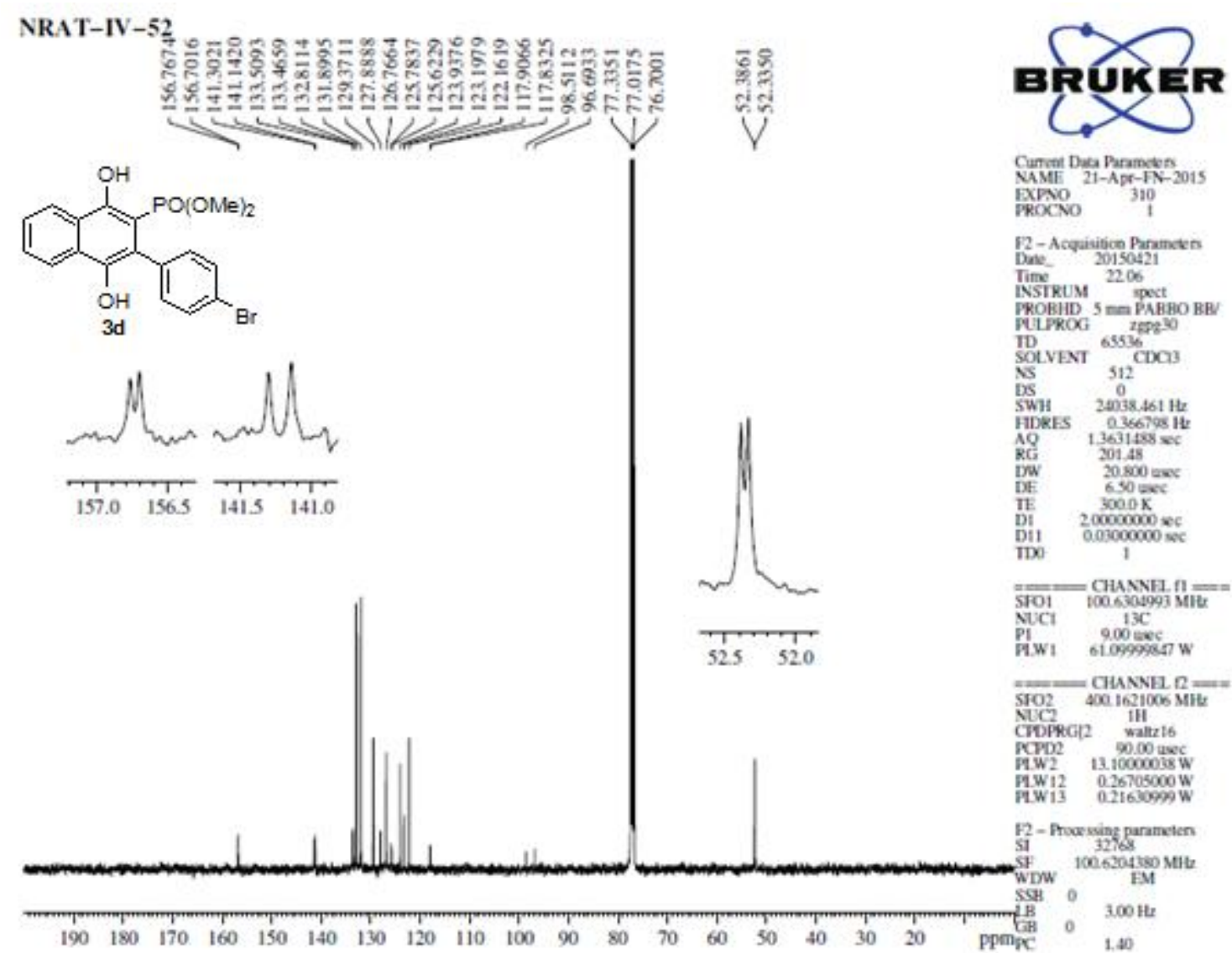

Figure 59: ${ }^{13} \mathrm{C}$ NMR spectrum of $3 \mathrm{~d}$
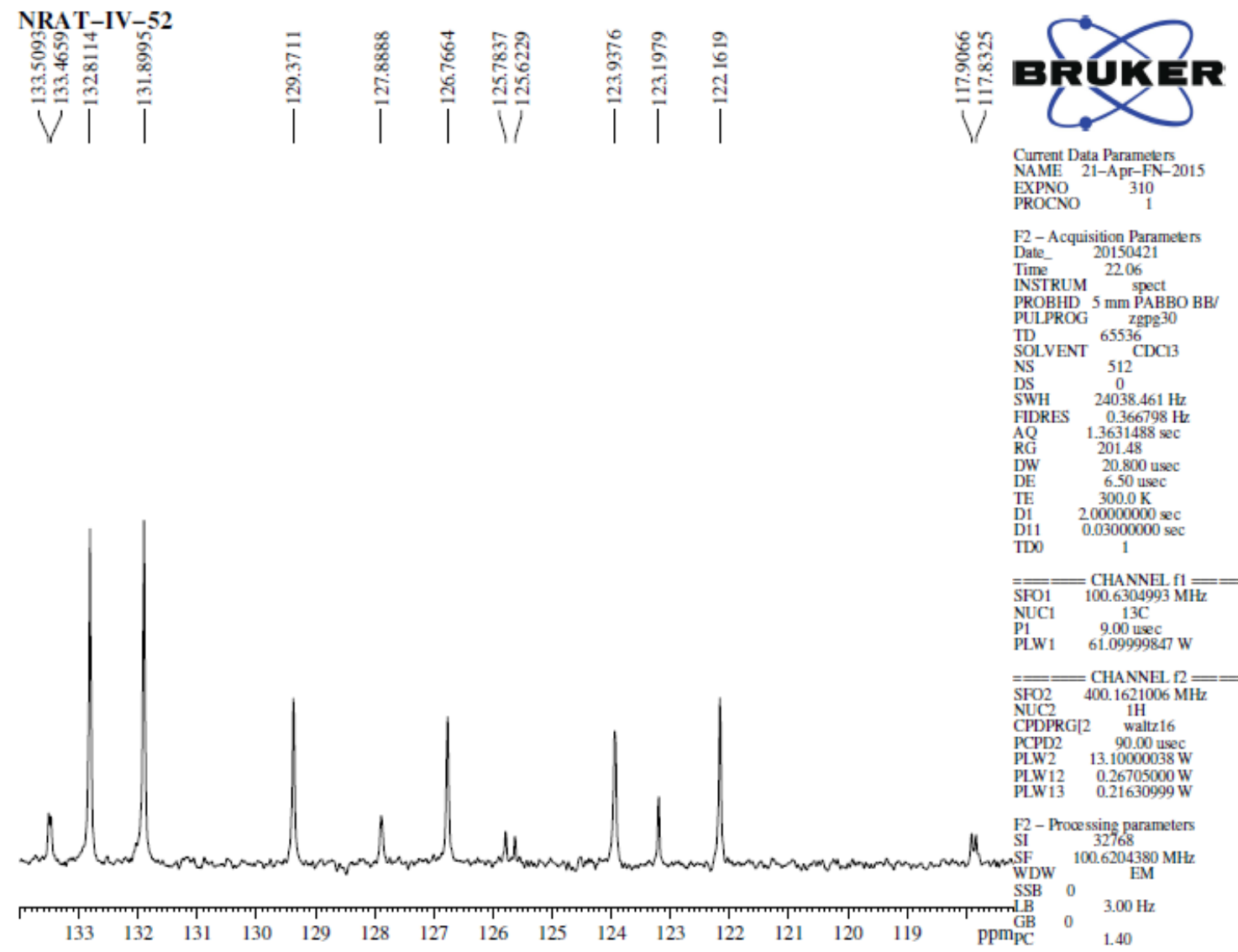

Figure 60: ${ }^{13} \mathrm{C}$ NMR spectrum of $3 \mathrm{~d}$ (expansion) 
NR-AT-IV-52

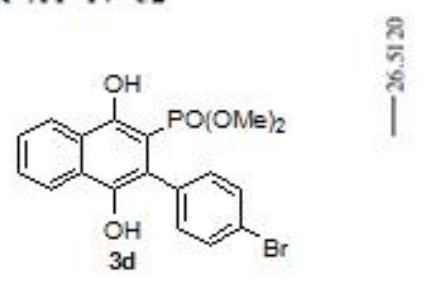

BRUKER

Cunge Data Pramesn $21-A p-A N-2015$

Expo

PROCNO 1

F2-Acquisibon Prameten

Time $\quad 7.29$

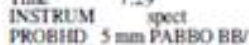

PROBHAD $5 \mathrm{~mm}$ PABBO BE

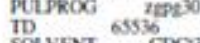

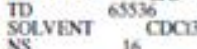

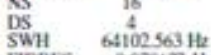

HIDRES $0.985121 \mathrm{HL}$

AO 0 OS111808

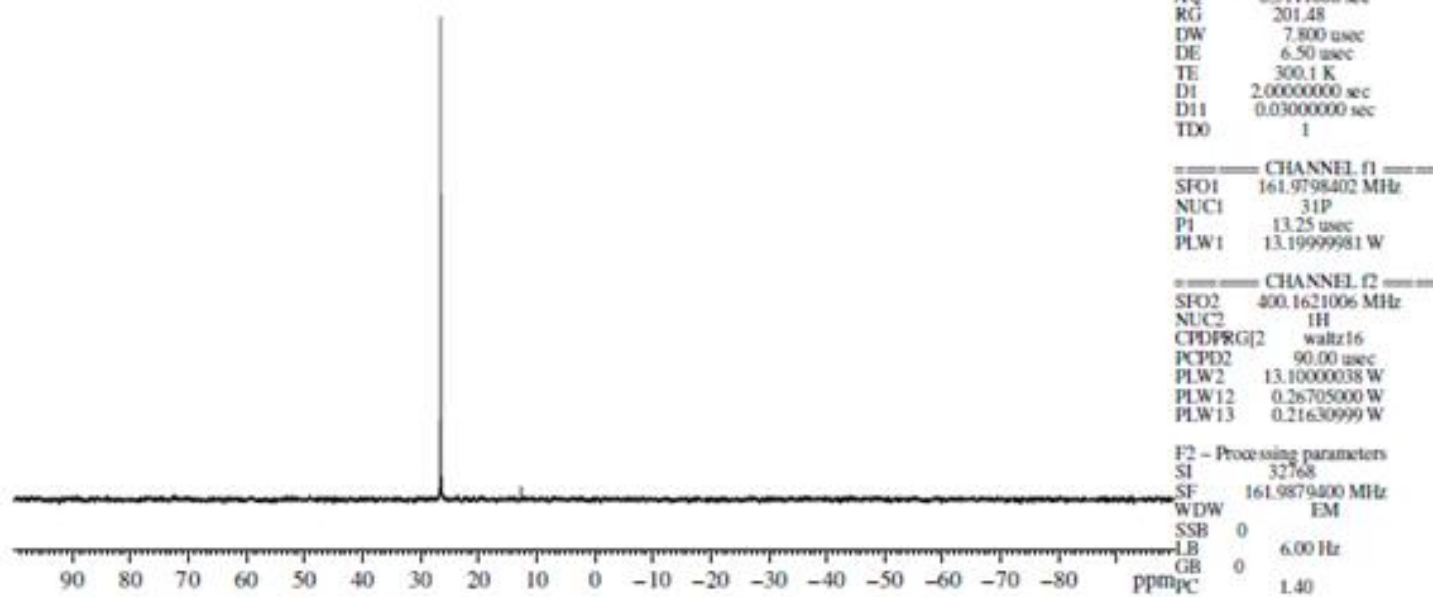

Figure 61: ${ }^{31} \mathrm{P}$ NMR spectrum of $3 d$

NRAT-IV-66

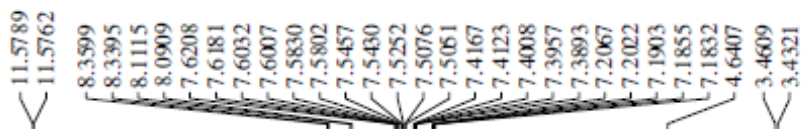<smiles>COc1c(O)c(-c2ccc(Cl)cc2)c(O)c2ccccc12</smiles>

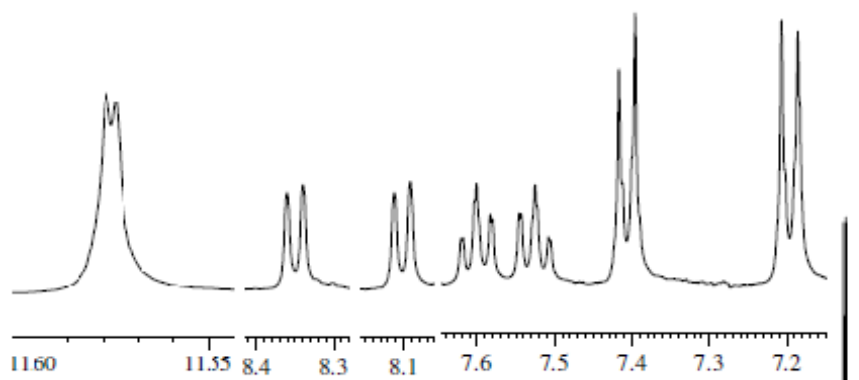
1.558 .4
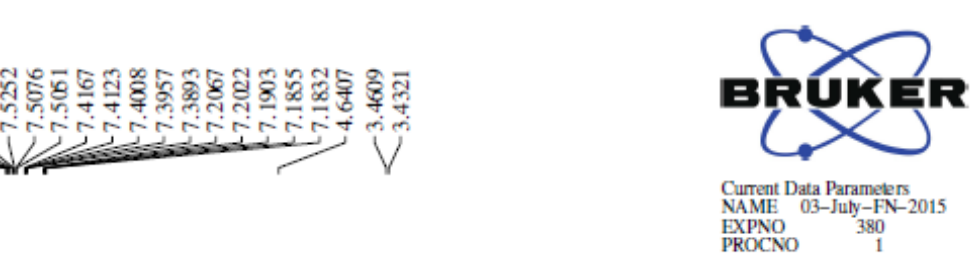

Current Data Parameters EXPNO $\quad 380$

F2 - Acquisition Parameters

$\begin{array}{cc}\text { Date_ } & 20150703 \\ \text { Dime } & 19.17\end{array}$

INSTRUM spect

$\underset{\text { PULPROG }}{\text { PROBHD }} 5 \mathrm{~mm} \underset{\mathrm{zg} 30}{\mathrm{PABBO}}$

TD $65536^{2}$

SOLVENT ${ }^{65536} \mathrm{CDCl}$

DS $\quad 00$

$\begin{array}{lr}\text { SWH } & 9615.385 \mathrm{~Hz} \\ \text { FIDRES } & 0.146719 \mathrm{~Hz}\end{array}$

AQ $\quad 3.4078720 \mathrm{sec}$

$\begin{array}{ll}\text { RG } & 114.26 \\ \text { DWV } & 52000\end{array}$

$\begin{array}{ll}\text { DW } & 52.000 \text { usec } \\ \text { DE } & 6.50 \text { usec }\end{array}$

TE $300.0 \mathrm{~K}$

D1

$=$ CHANNEL $\mathrm{f1}=$

SFO1 $400.1629712 \mathrm{MH}$

$\begin{array}{ll}\text { P1 } & 1285 \text { usec } \\ \text { PLW1 } & 13.10000038 \mathrm{~W}\end{array}$

F2 - Processing parameters

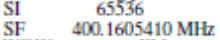

SF $400.1605410 \mathrm{M}$

$\begin{array}{lll}\mathrm{SSB} & 0 & 0.30 \mathrm{~Hz}\end{array}$

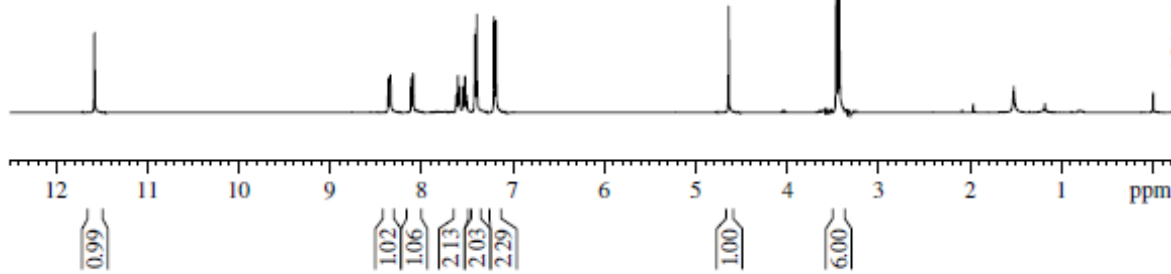

1.00

Figure 62: ${ }^{1} \mathrm{H}$ NMR spectrum of $3 e$ 


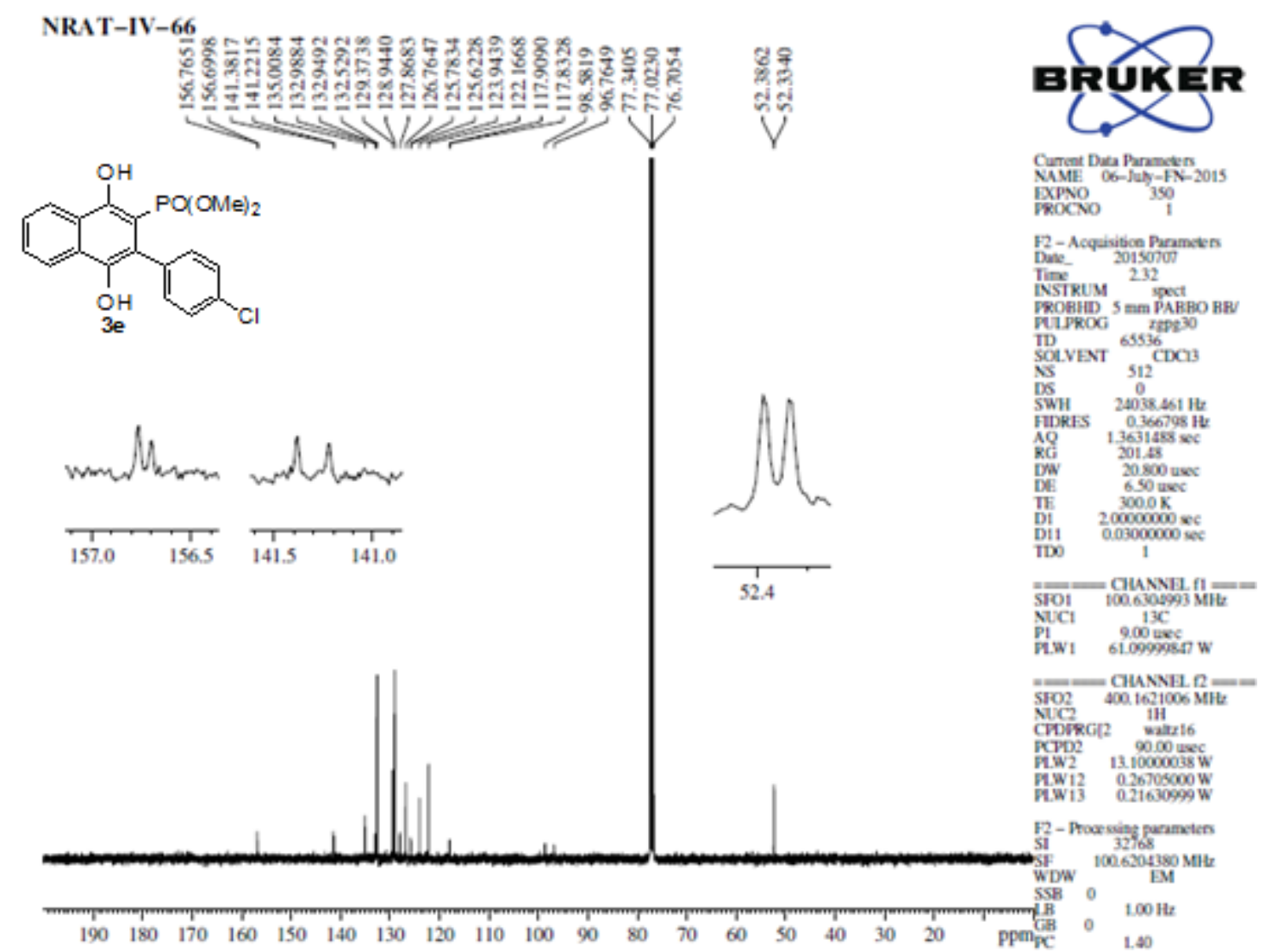

Figure 63: ${ }^{13} \mathrm{C}$ NMR spectrum of $3 \mathrm{e}$

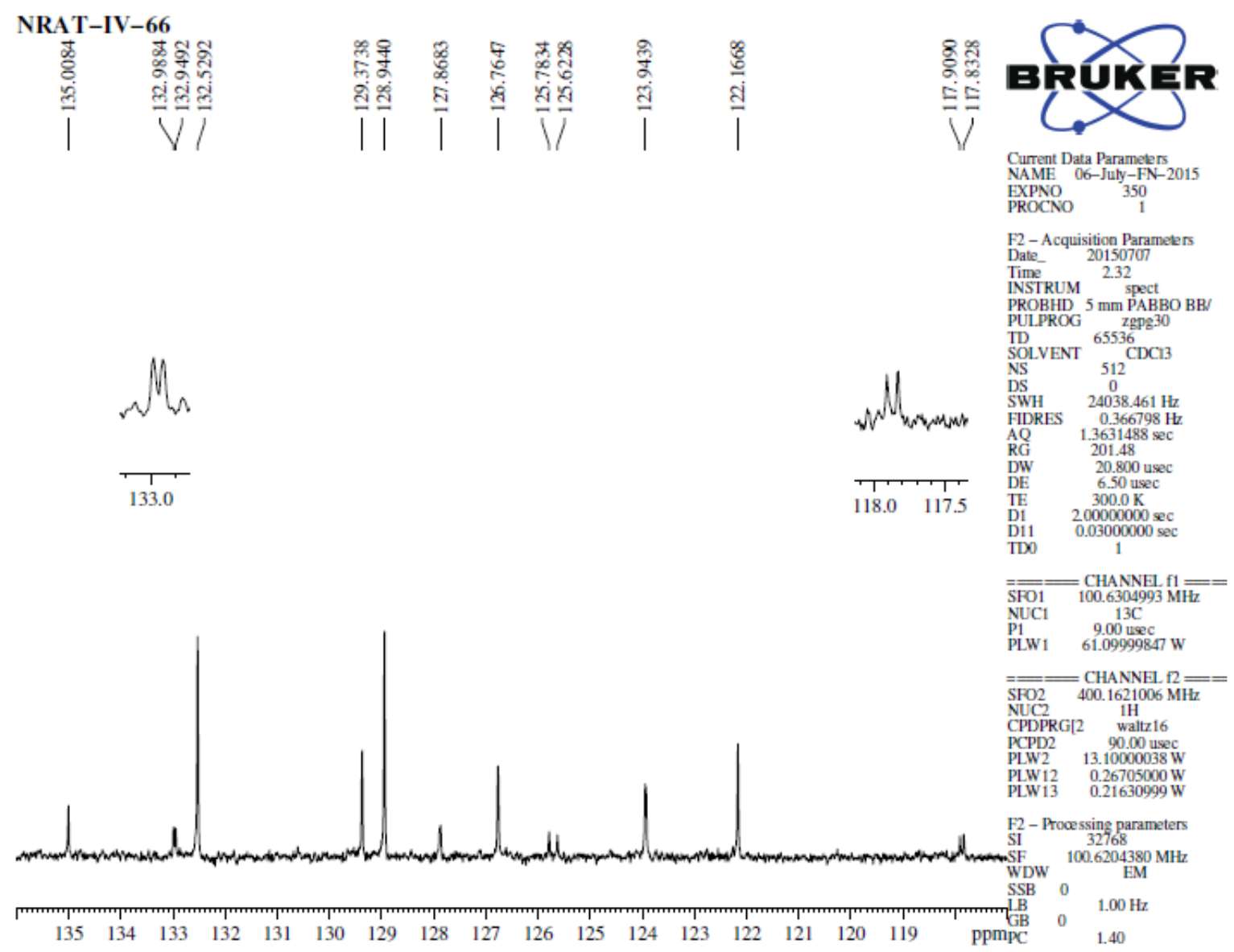

Figure 64: ${ }^{13} \mathrm{C}$ NMR spectrum of $3 \mathrm{e}$ (expansion) 


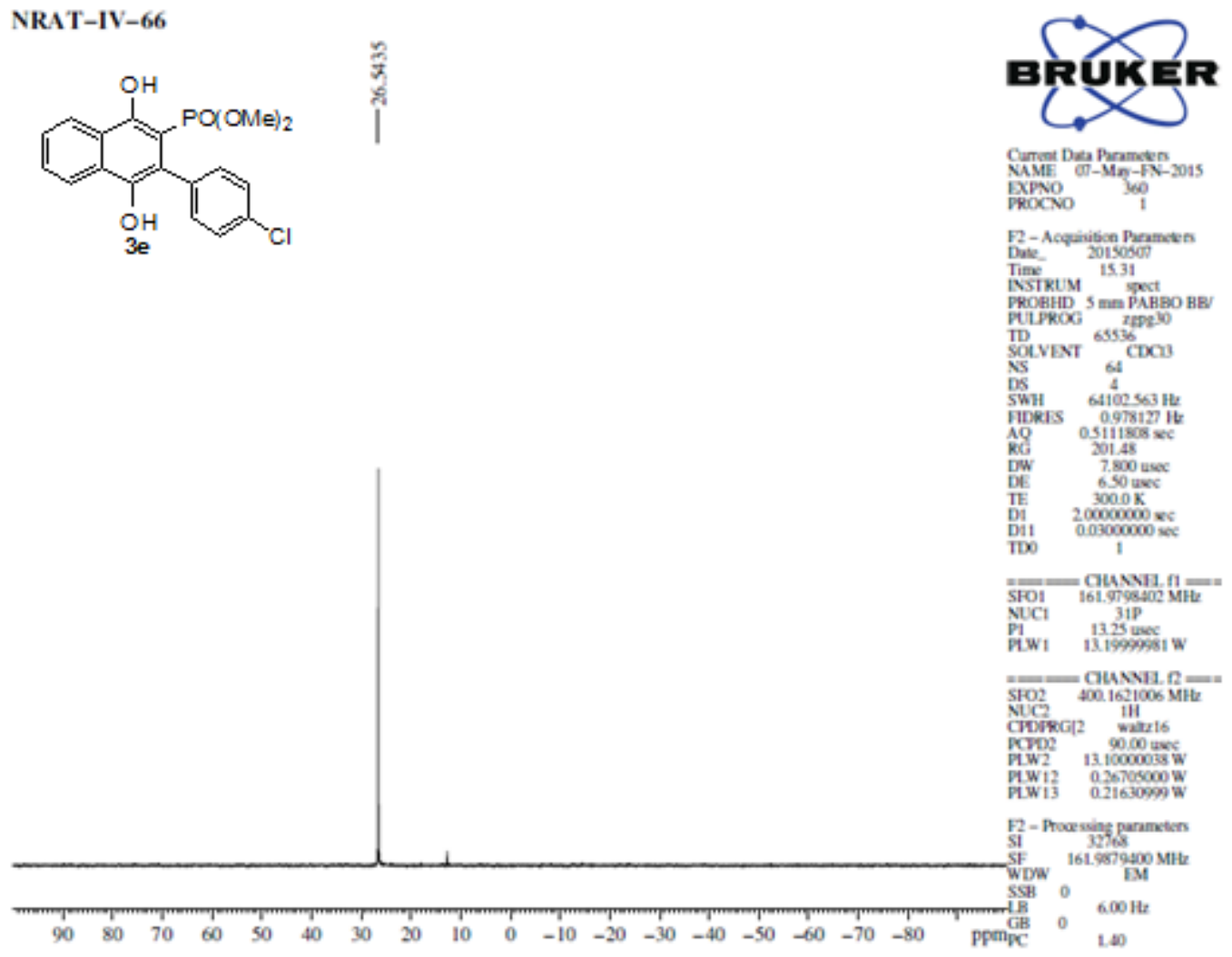

Figure 65: ${ }^{31} \mathrm{P}$ NMR spectrum of $3 \mathrm{e}$

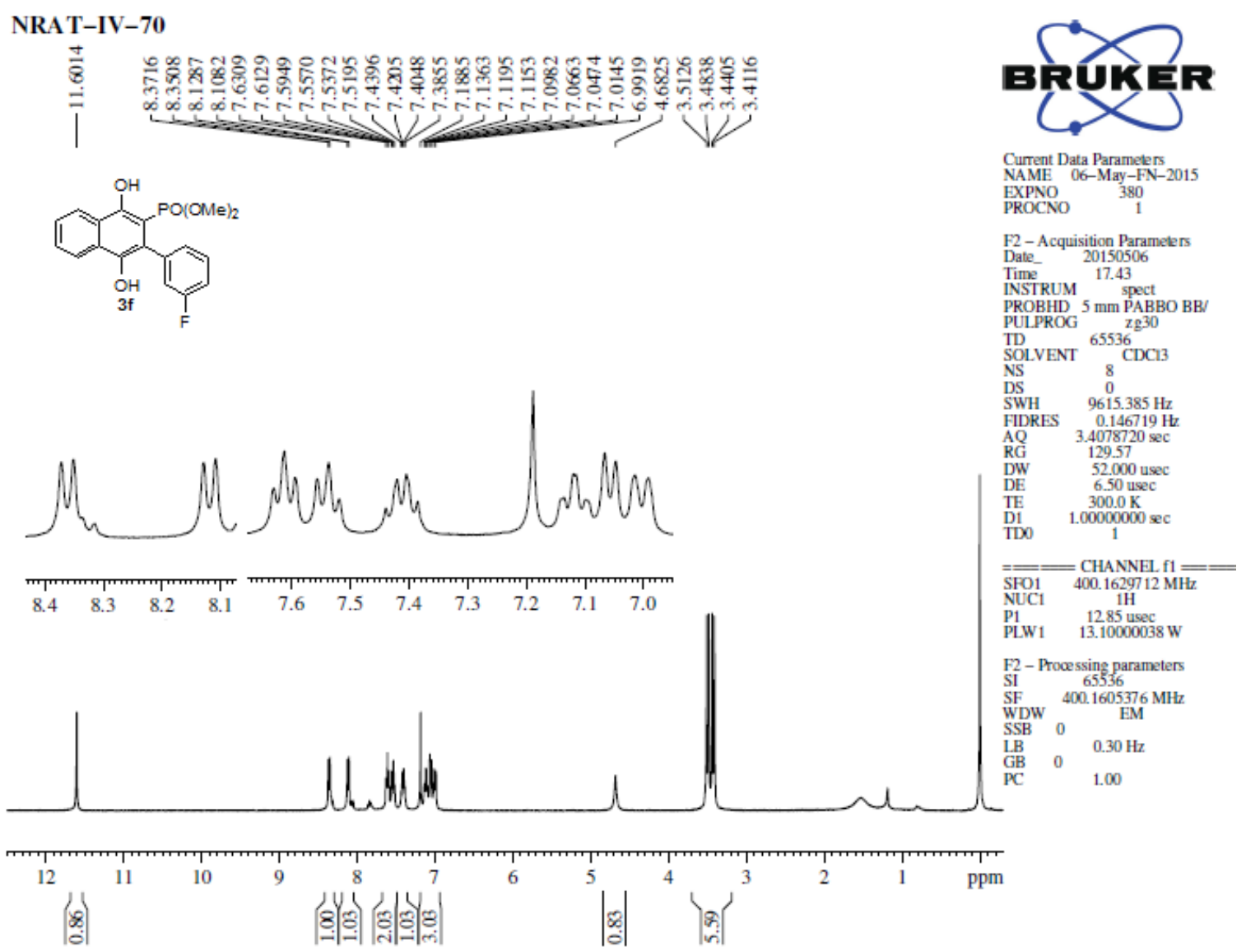

Figure 66: ${ }^{1} \mathrm{H}$ NMR spectrum of $3 f$ 


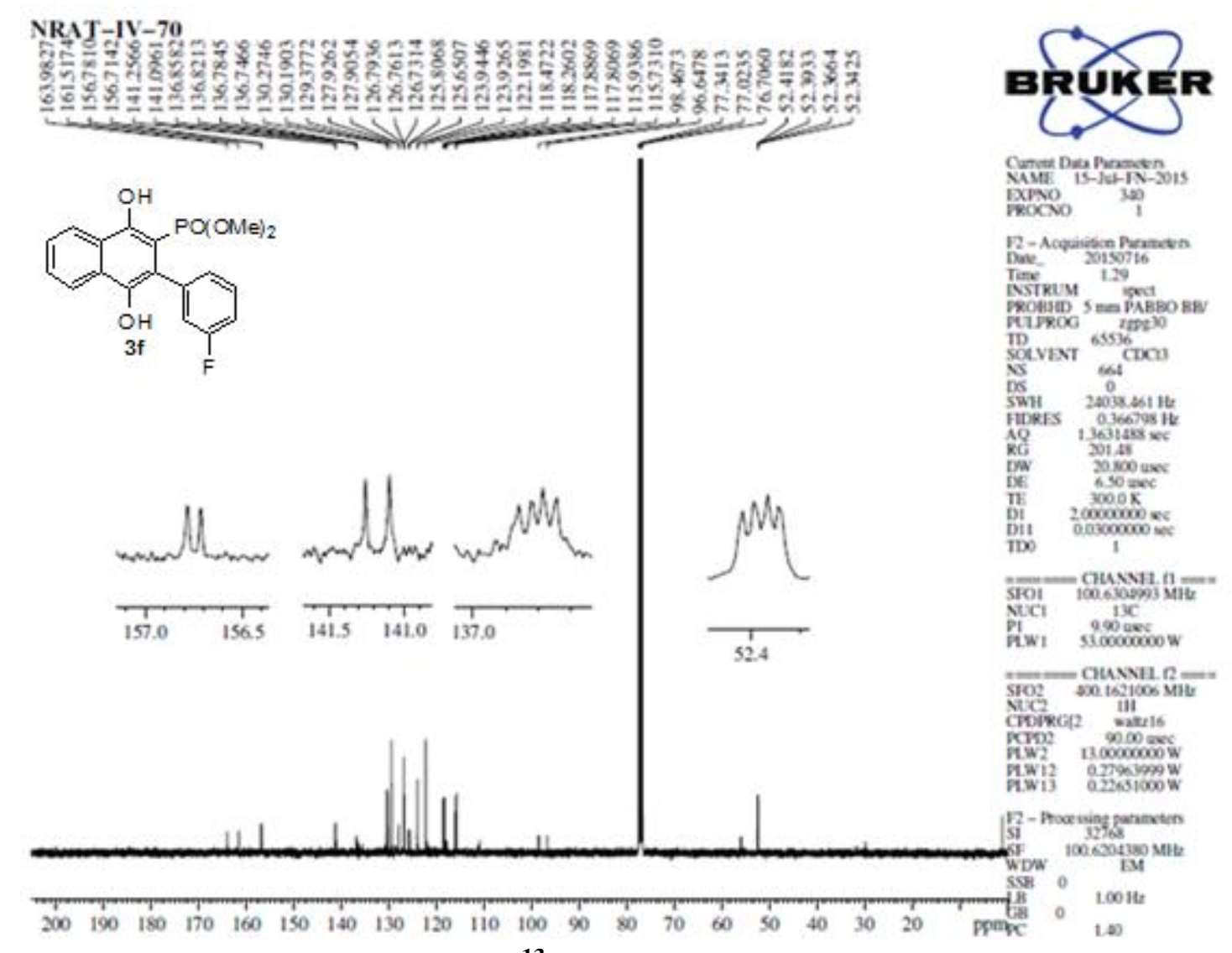

Figure 67: ${ }^{13} \mathrm{C}$ NMR spectrum of $3 f$

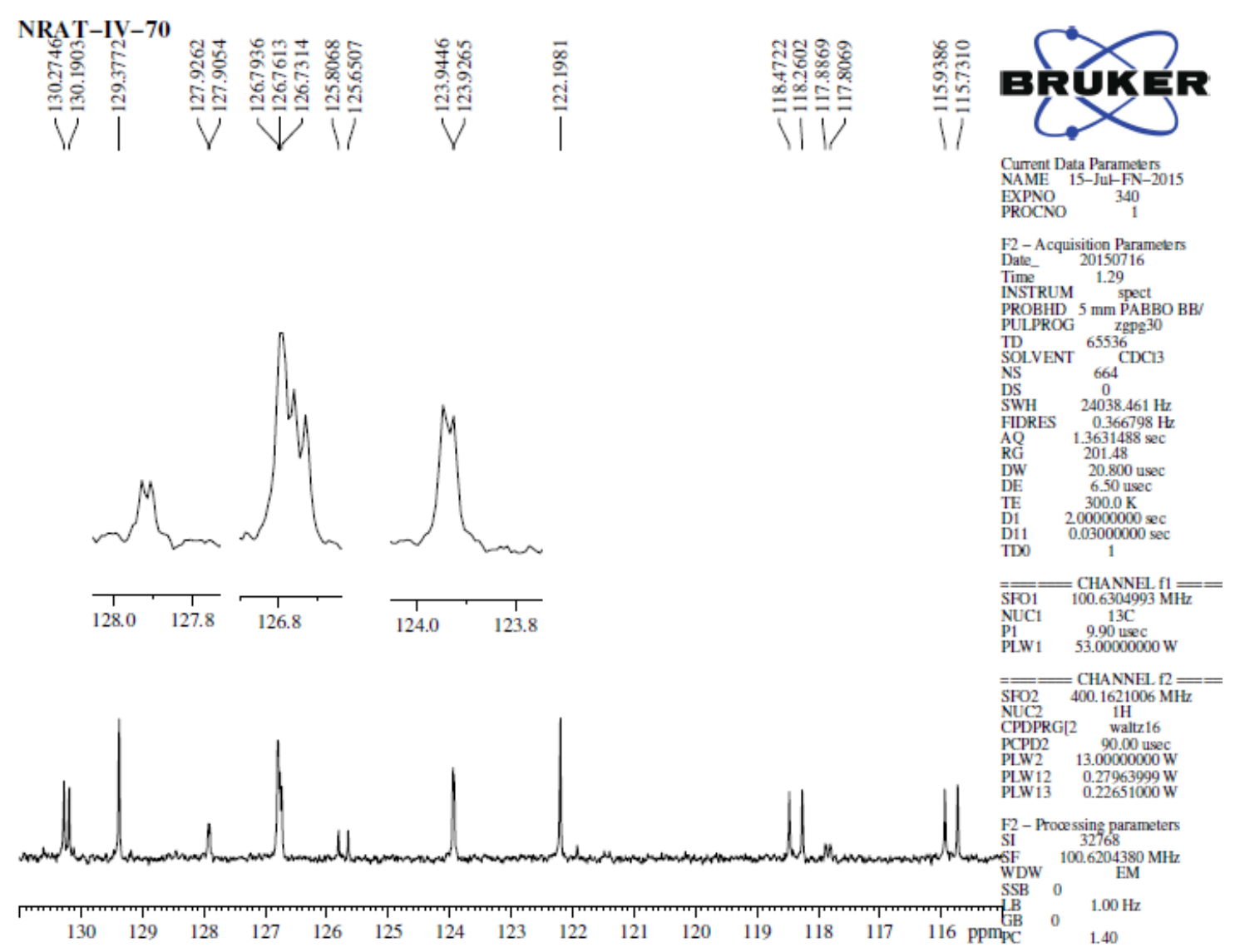

Figure 68: ${ }^{13} \mathrm{C}$ NMR spectrum of $3 f$ (expansion) 


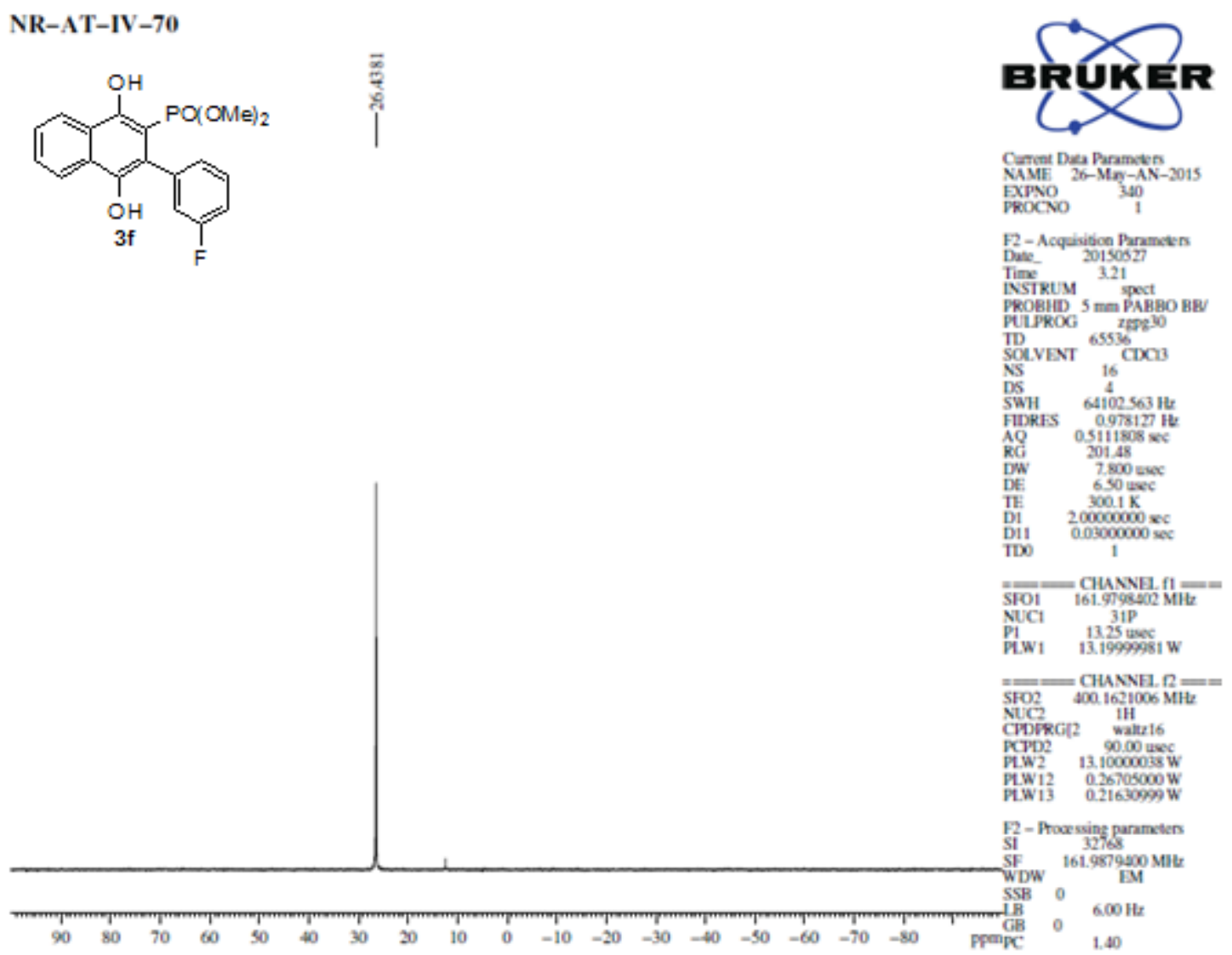

Figure 69: ${ }^{31} \mathrm{P}$ NMR spectrum of $3 f$

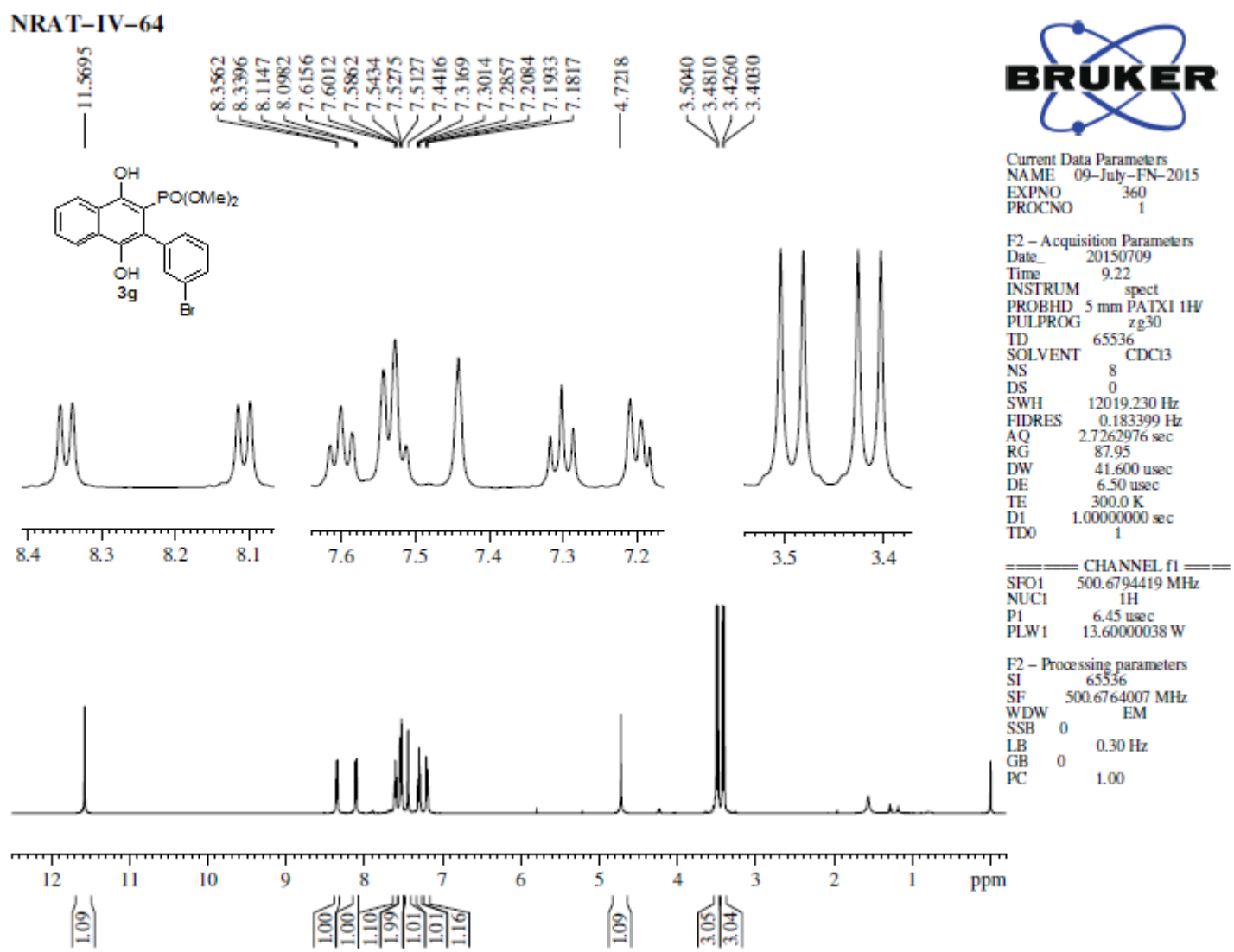

Figure 70: ${ }^{1} \mathrm{H}$ NMR spectrum of $\mathbf{3 g}$ 


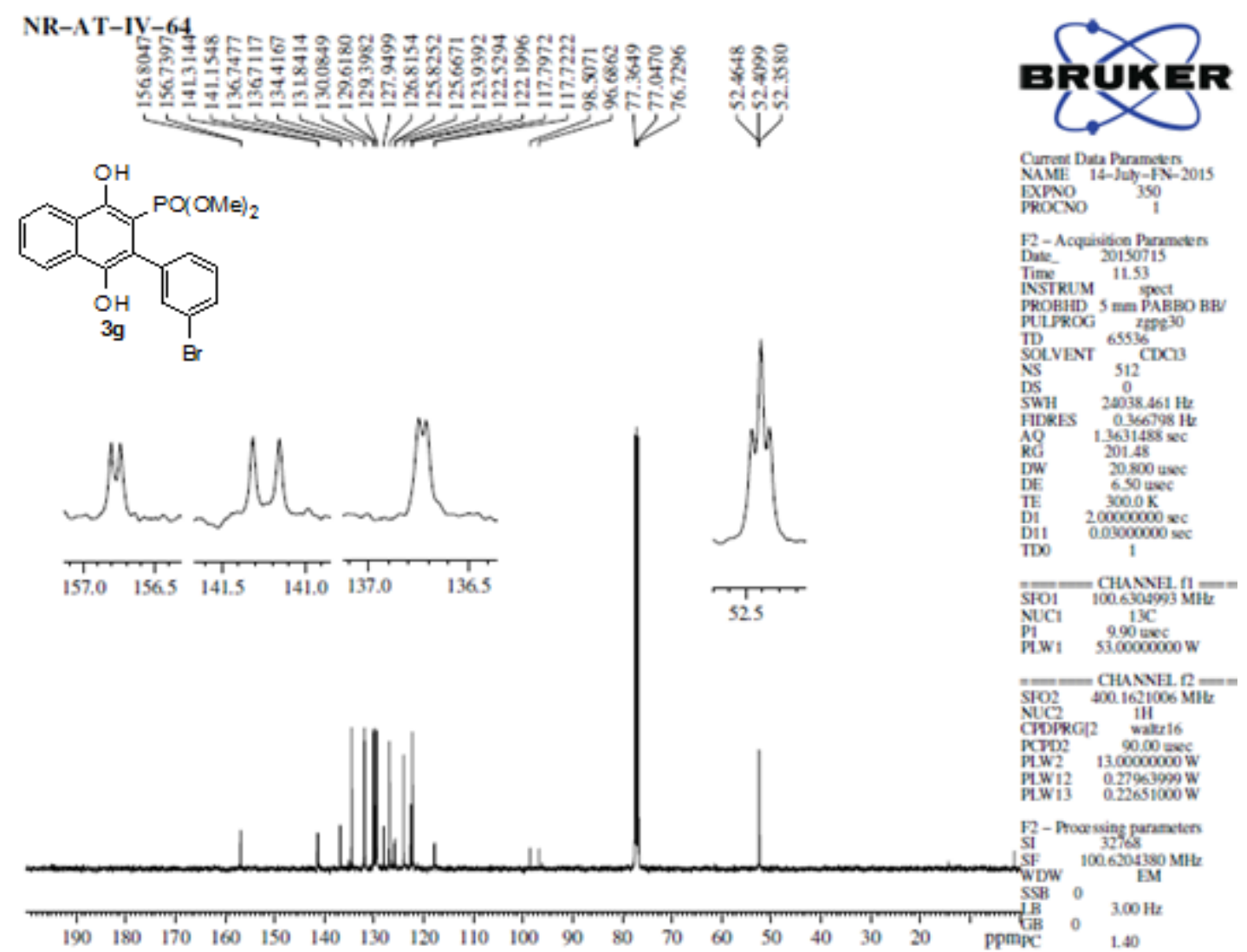

Figure 71: ${ }^{13} \mathrm{C}$ NMR spectrum of $3 \mathrm{~g}$

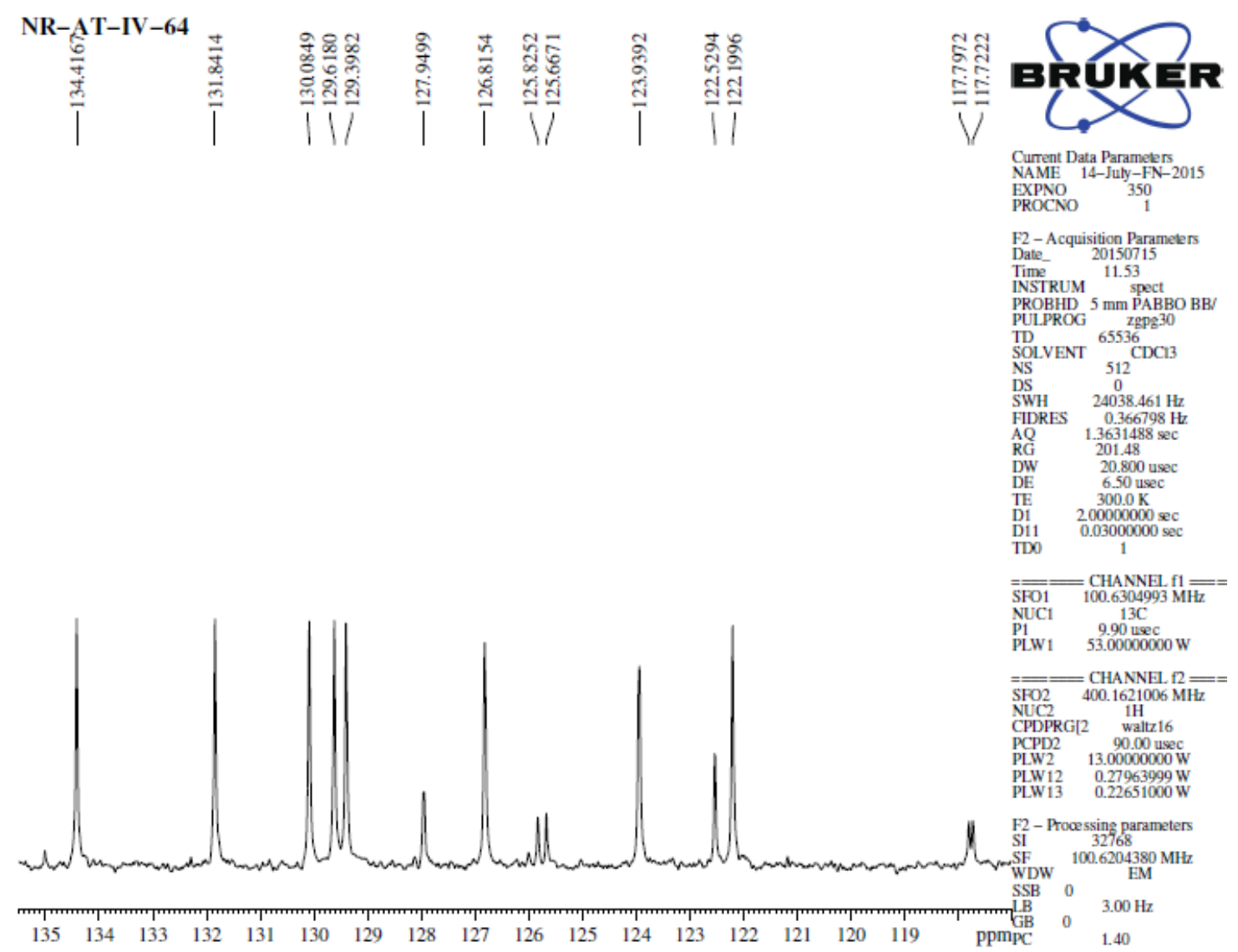

Figure 72: ${ }^{13} \mathrm{C}$ NMR spectrum of $3 \mathrm{~g}$ (expansion) 


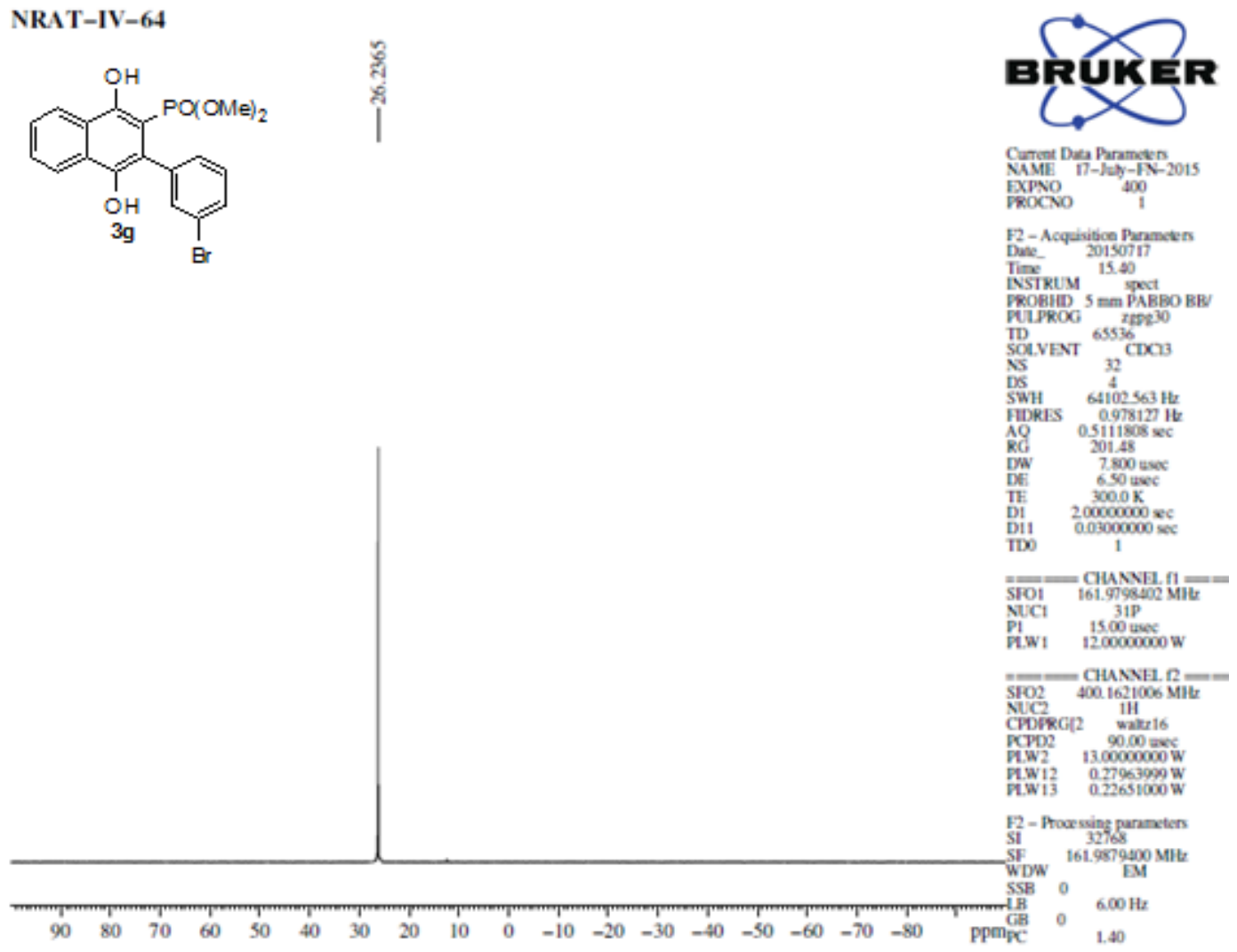

Figure 73: ${ }^{31} \mathrm{P}$ NMR spectrum of $3 \mathrm{~g}$

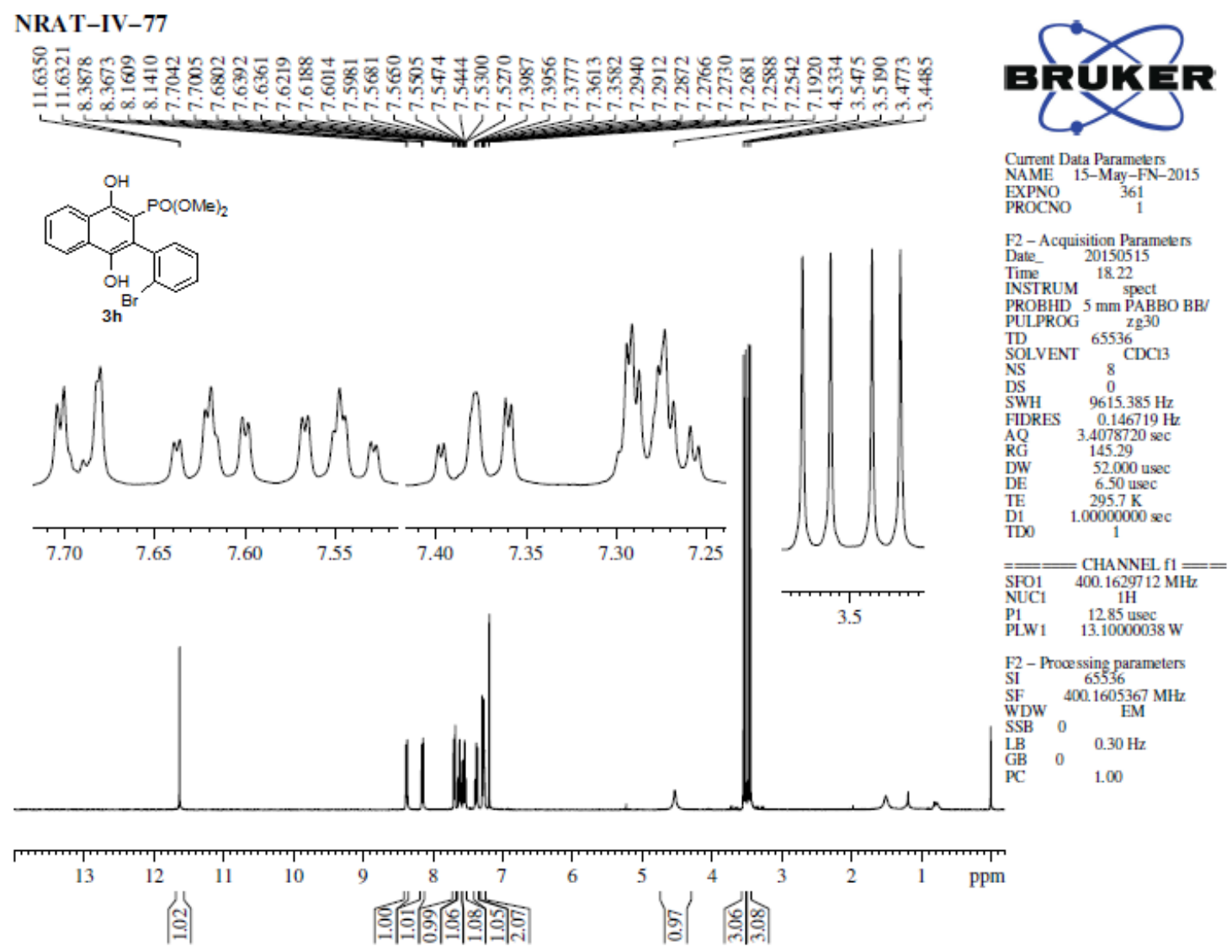

Figure 74: ${ }^{1} \mathrm{H}$ NMR spectrum of $3 \mathrm{~h}$ 


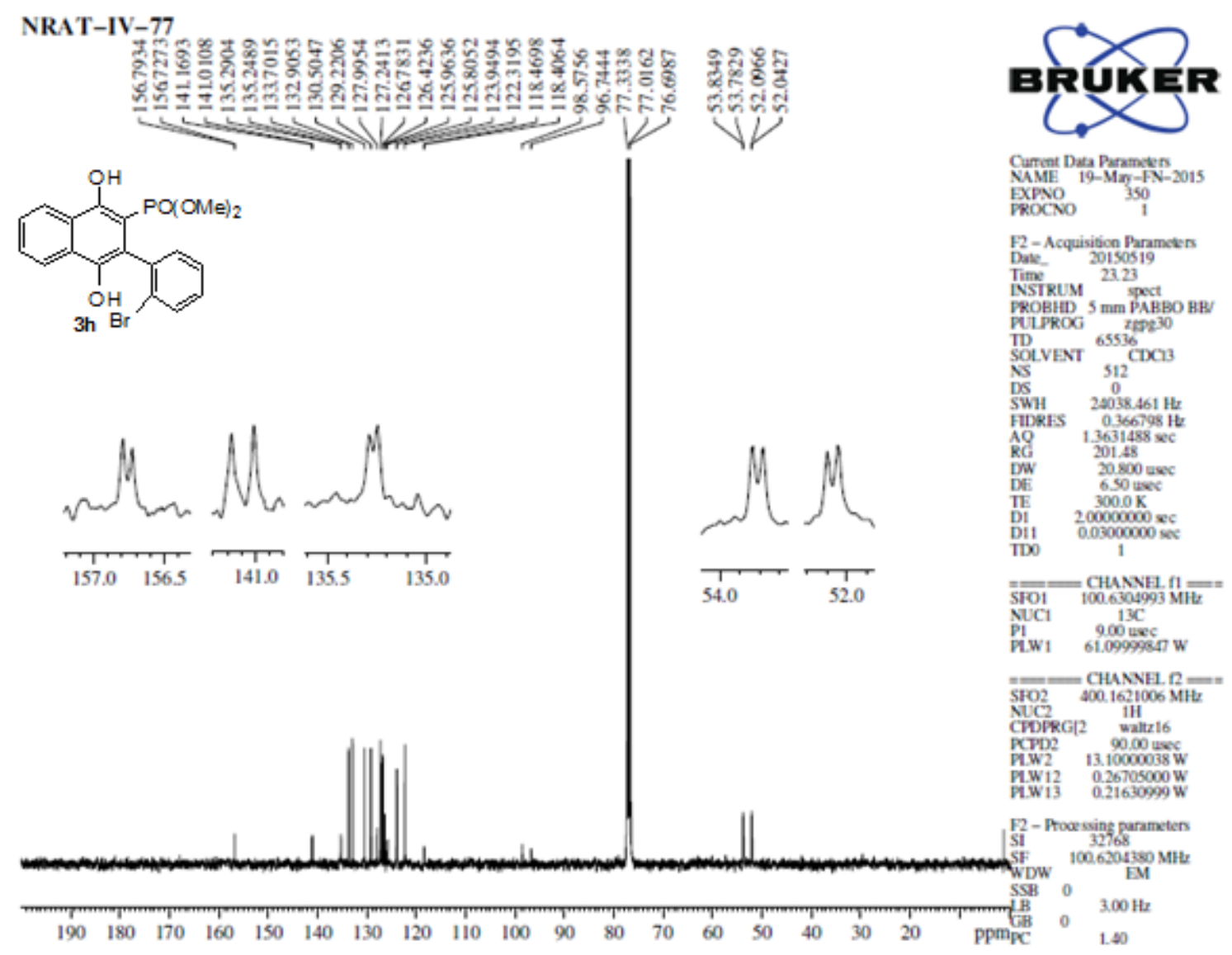

Figure 75: ${ }^{13} \mathrm{C}$ NMR spectrum of $3 \mathrm{~h}$
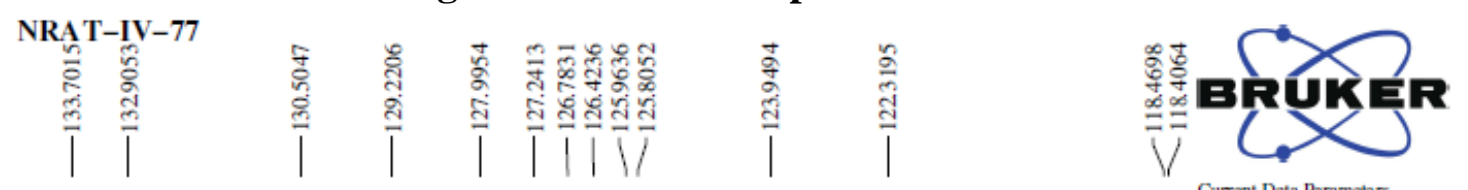

Current Data Parameters
NAME
$19-$ May-FN-2015 $\begin{array}{lr}\text { NAME } & 19-\text { May-FN-20 } \\ \text { PROCNO } & 350\end{array}$

F2 - Acquisition Parameters

Date__ 20150519

Time 23.23

PROBHD 5 mm PABBO BB/

PULPROG ${ }_{\text {T.PPg } 30}$

TDOLVENT ${ }^{65536} \mathrm{CDCl}$

$\begin{array}{lc}\text { NS } & 512 \\ \text { DS } & 0\end{array}$

$\begin{array}{lc}\text { DS } & 0 \\ \text { SWH } & 24038.461 \mathrm{~Hz}\end{array}$

FIDRES $\quad 0.366798 \mathrm{H}$

$\begin{array}{ll}\text { AQ } & 1.363148 \\ \text { RG } & 201.48\end{array}$

$\begin{array}{ll}\text { DW } & 20.800 \text { usec } \\ \text { DE } & 6.50 \text { usec }\end{array}$

$6.50 \mathrm{usec}$
$300.0 \mathrm{~K}$

$200000000 \mathrm{sec}$

D11 $\quad 0.03000000 \mathrm{sec}$

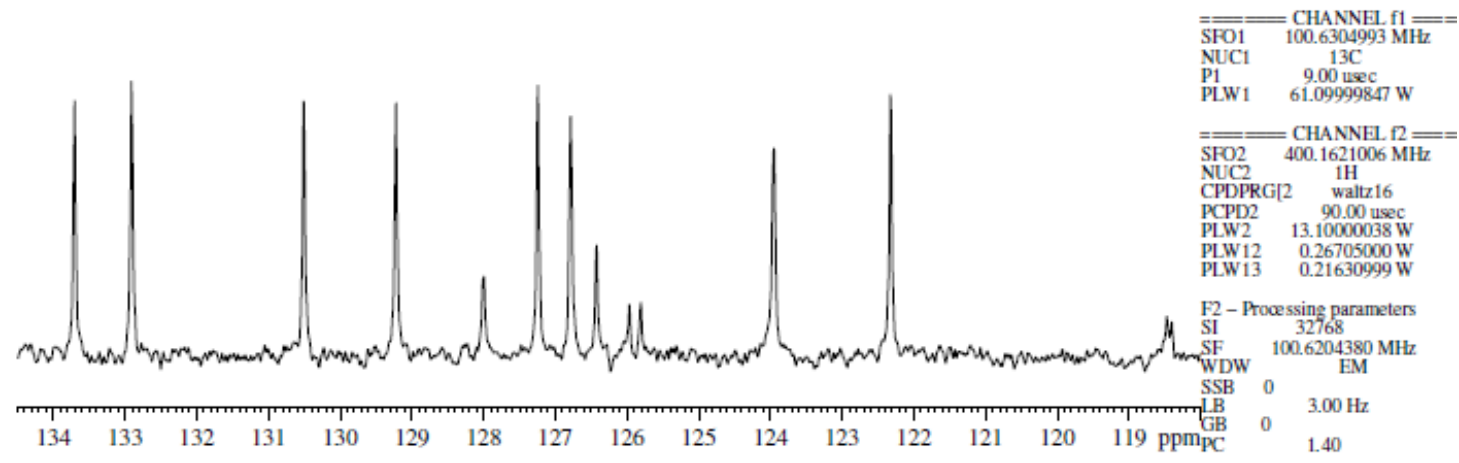

Figure 76: ${ }^{13} \mathrm{C}$ NMR spectrum of $3 \mathrm{~h}$ (expansion) 


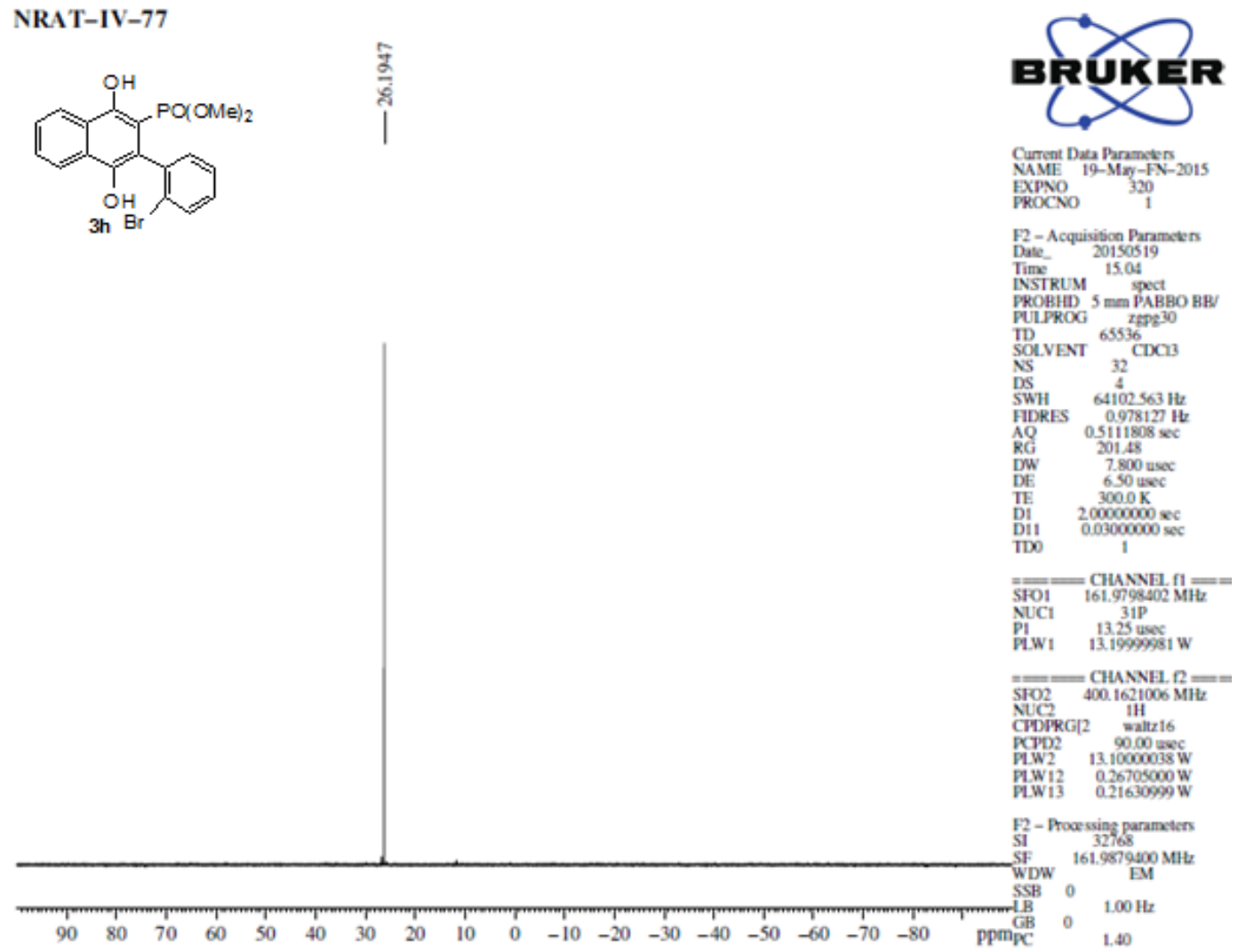

Figure 77: ${ }^{31} \mathrm{P}$ NMR spectrum of $3 \mathrm{~h}$

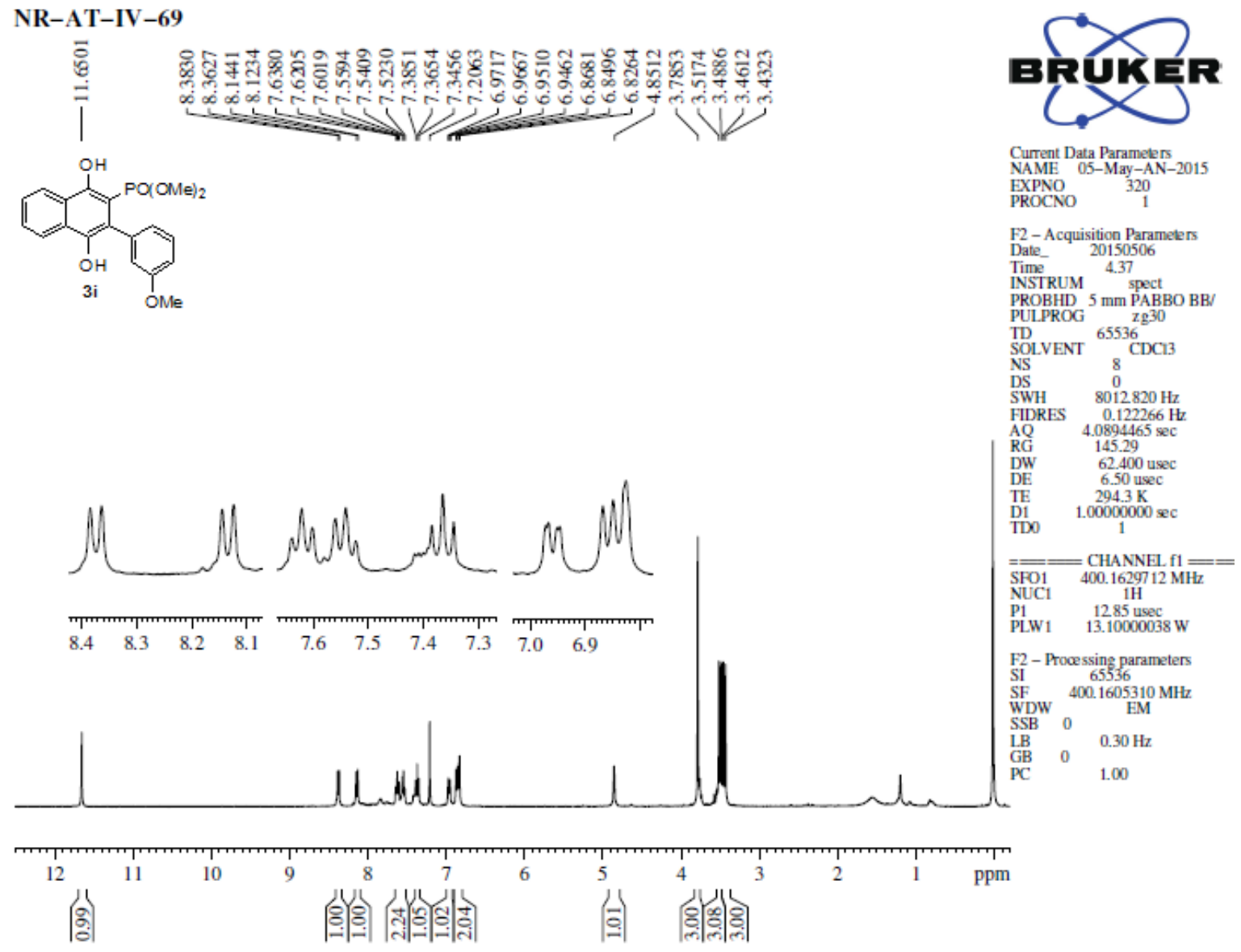

Figure 78: ${ }^{1} \mathrm{H}$ NMR spectrum of $3 \mathrm{i}$ 


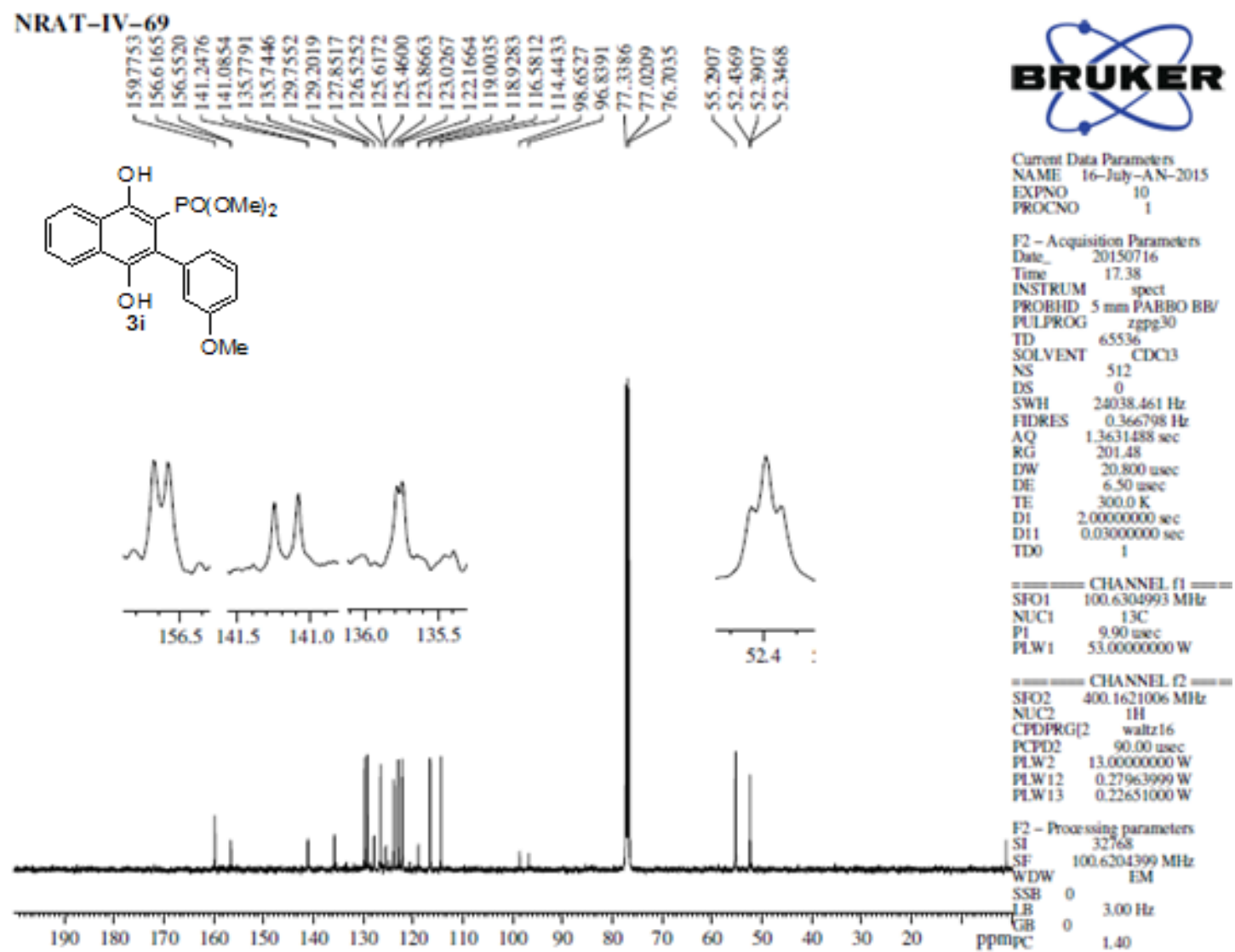

Figure 79: ${ }^{13} \mathrm{C}$ NMR spectrum of $3 \mathrm{i}$

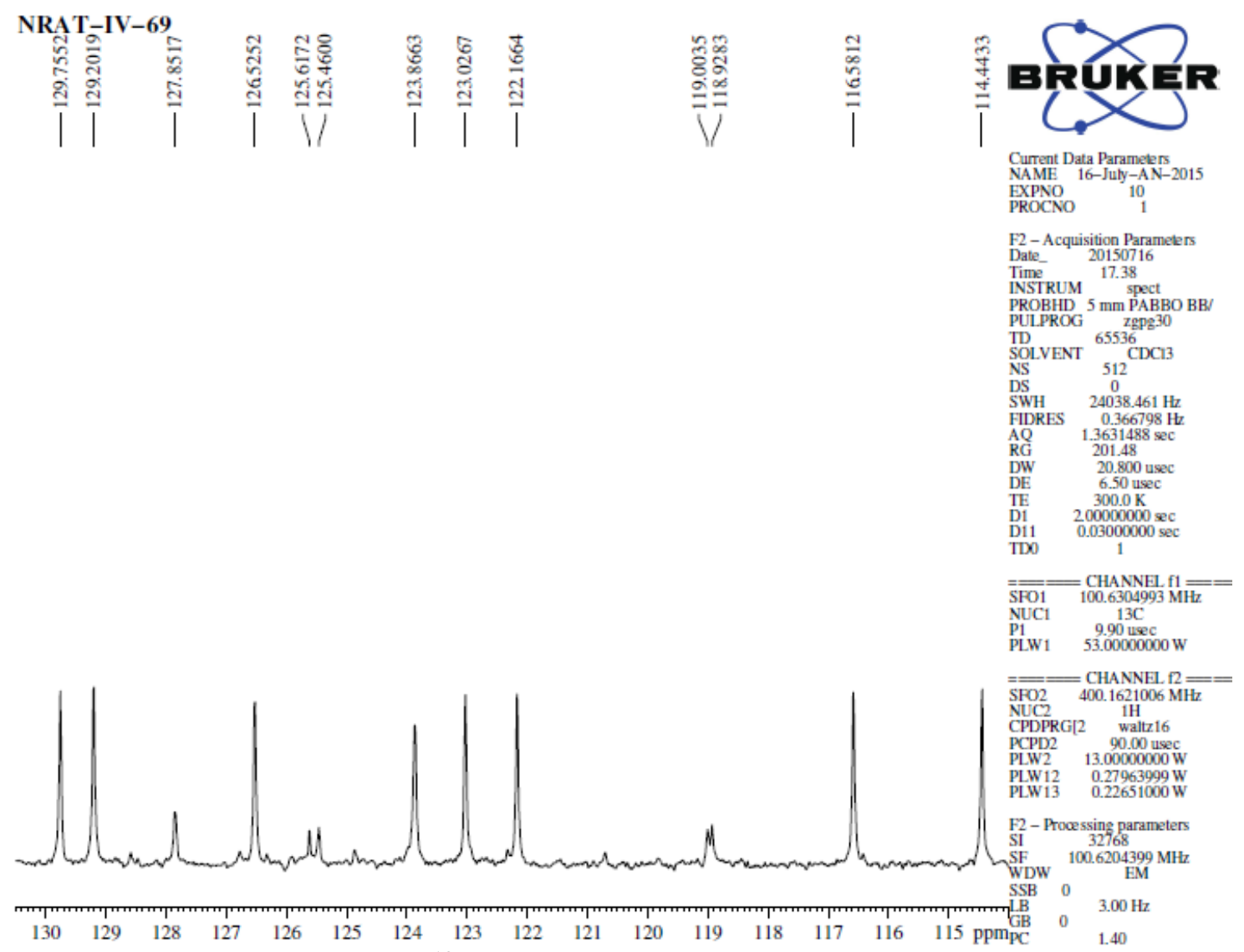

Figure 80: ${ }^{13} \mathrm{C}$ NMR spectrum of $3 \mathrm{i}$ (expansion) 
NR-AT-IV-69<smiles>COc1cccc(-c2c(O)c(O)c3ccccc3c2O)c1</smiles>

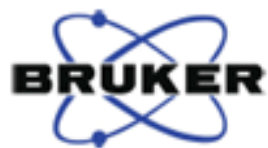

Cuncent Da Pramess EXPNO
PROCNO

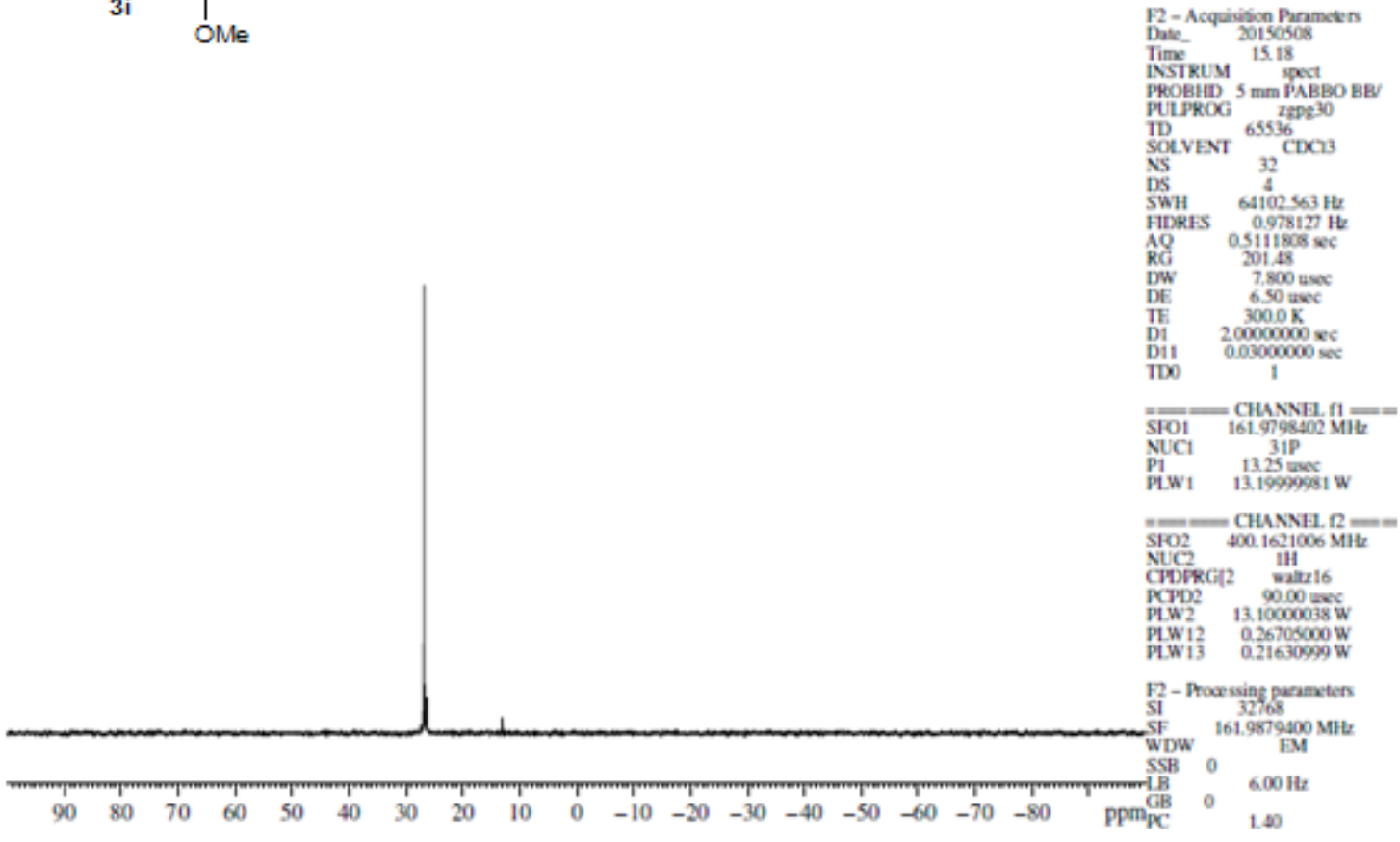

Figure 81: ${ }^{31} \mathrm{P}$ NMR spectrum of $3 \mathrm{i}$

NRAT-IV-71
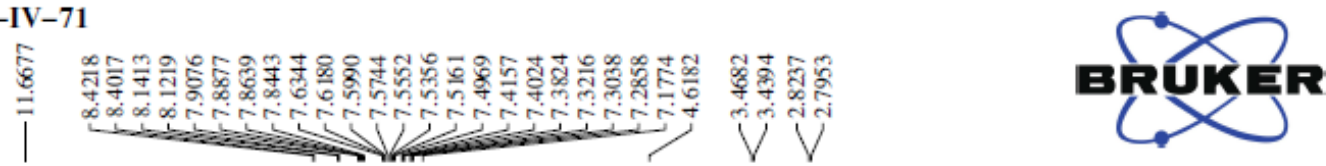<smiles>COC(=O)c1c(-c2cccc3ccccc23)c(O)c2ccccc2c1O</smiles>
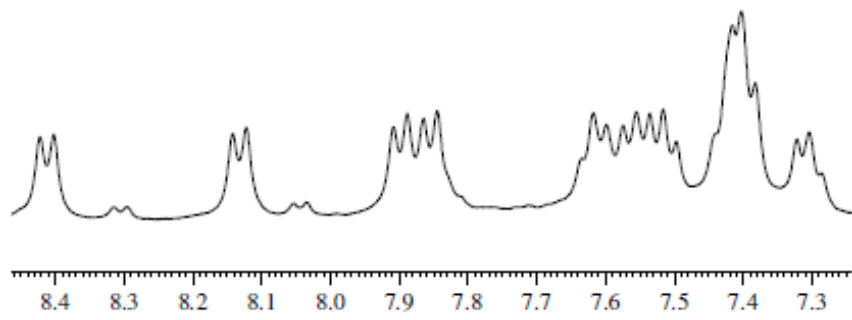

Current Data Paramelers
NAME $08-M a y-F N-2015$ EXPNO

F2 - Acquisition Parameters

$\begin{array}{cc}\text { Date_ } & 20150508 \\ \text { Time } & 15.06\end{array}$

INSTRUM 15.06 spect

PULPROG 5 mm PAB $\mathrm{zg}^{30}$

TD $_{\text {SOLVENT }}{ }^{65536}$ CDC13

$\begin{array}{ll}\text { NS } & 8 \\ \text { DS } & 0\end{array}$

$\begin{array}{lll}\text { DS } & 0 \\ \text { SWH } & 9615.385 \mathrm{~Hz}\end{array}$

$\begin{array}{lr}\text { FIDRES } & 0.146719 \mathrm{Ht} \\ \mathrm{AO} & 3.4078720 \mathrm{sec}\end{array}$

$\begin{array}{ll}\text { AQ } & 3.4078720 \mathrm{sec}\end{array}$

DW $\quad 52.000$ usec

TE

$\begin{array}{lc}\text { TE } & 300.0 \mathrm{~K} \\ \text { D1 } & 1.00000000 \mathrm{sec}\end{array}$

$=$ CHANNEL $\mathrm{f} 1=$

SUC1 $400.1629712 \mathrm{MH}$

$\begin{array}{ll}\text { P1 } & 12.85 \text { usec } \\ \text { PLW1 } & 13.10000038 \text { W }\end{array}$

F2 - Procssing parameters

$\begin{array}{ll}\text { SI } & 65536 \\ \text { SF } & 400.1605422 \mathrm{MHz}\end{array}$

$\begin{array}{ll}\text { WDW } & 400.1605422 \\ \text { SSB } & 0\end{array}$

$\begin{array}{lll}\mathrm{LSB} & 0 & 0.30 \mathrm{~Hz} \\ \mathrm{~GB} & 0\end{array}$

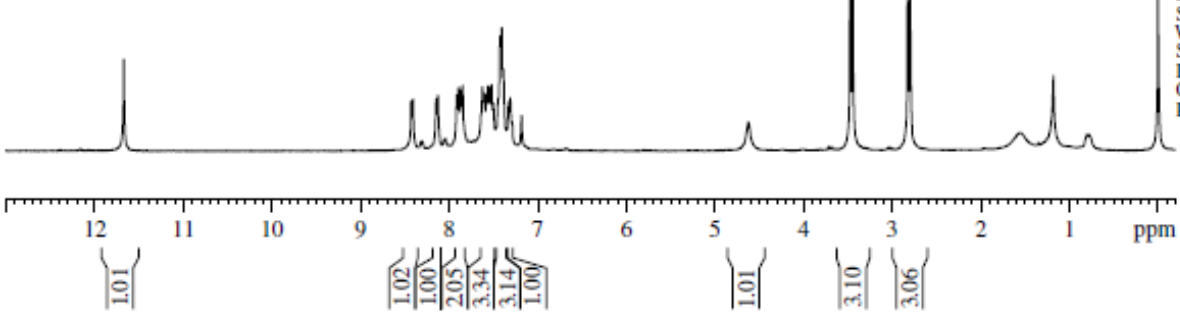

1.00

Figure 82: ${ }^{1} \mathrm{H}$ NMR spectrum of $3 \mathrm{j}$ 


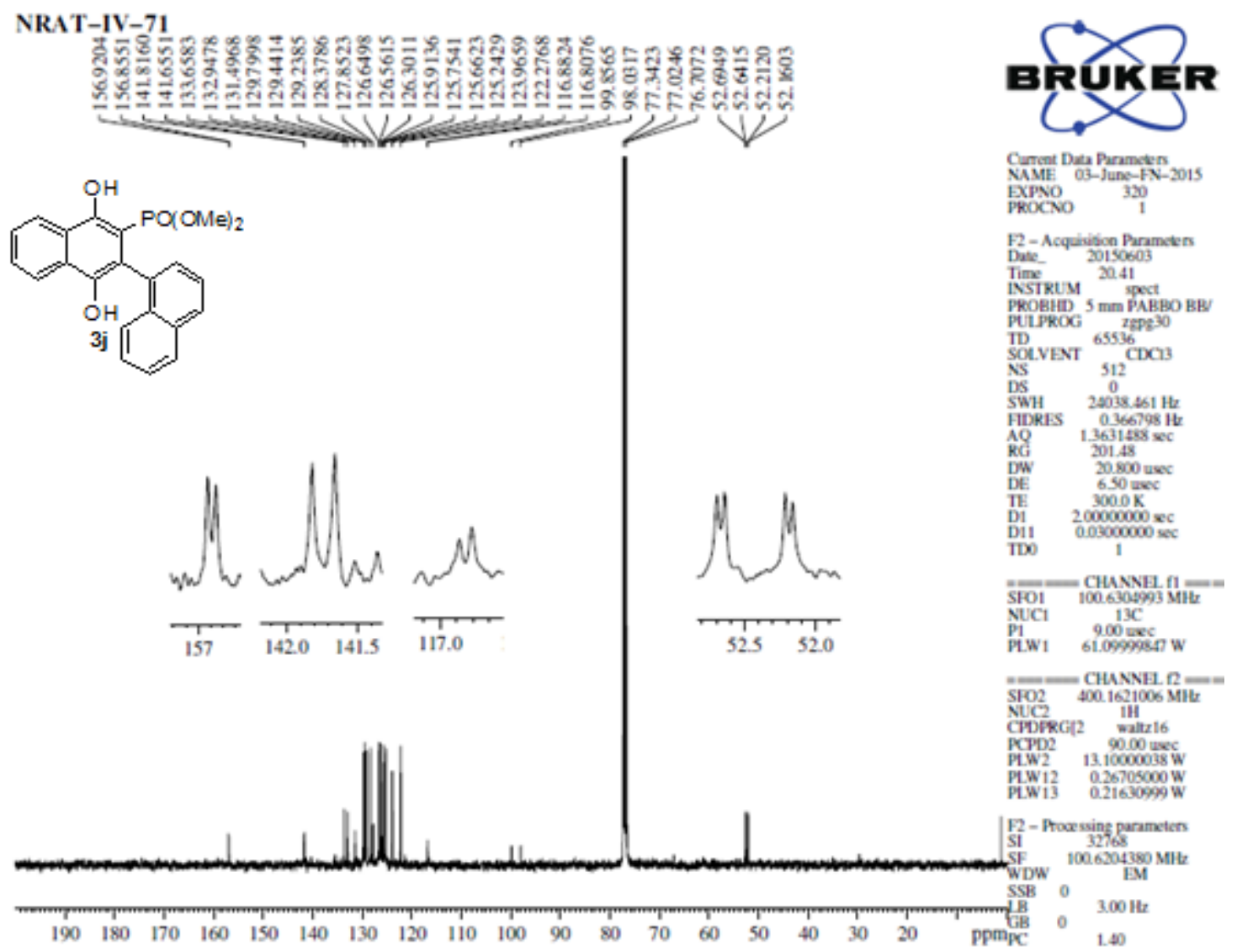

Figure 83: ${ }^{13} \mathrm{C}$ NMR spectrum of $3 \mathbf{j}$

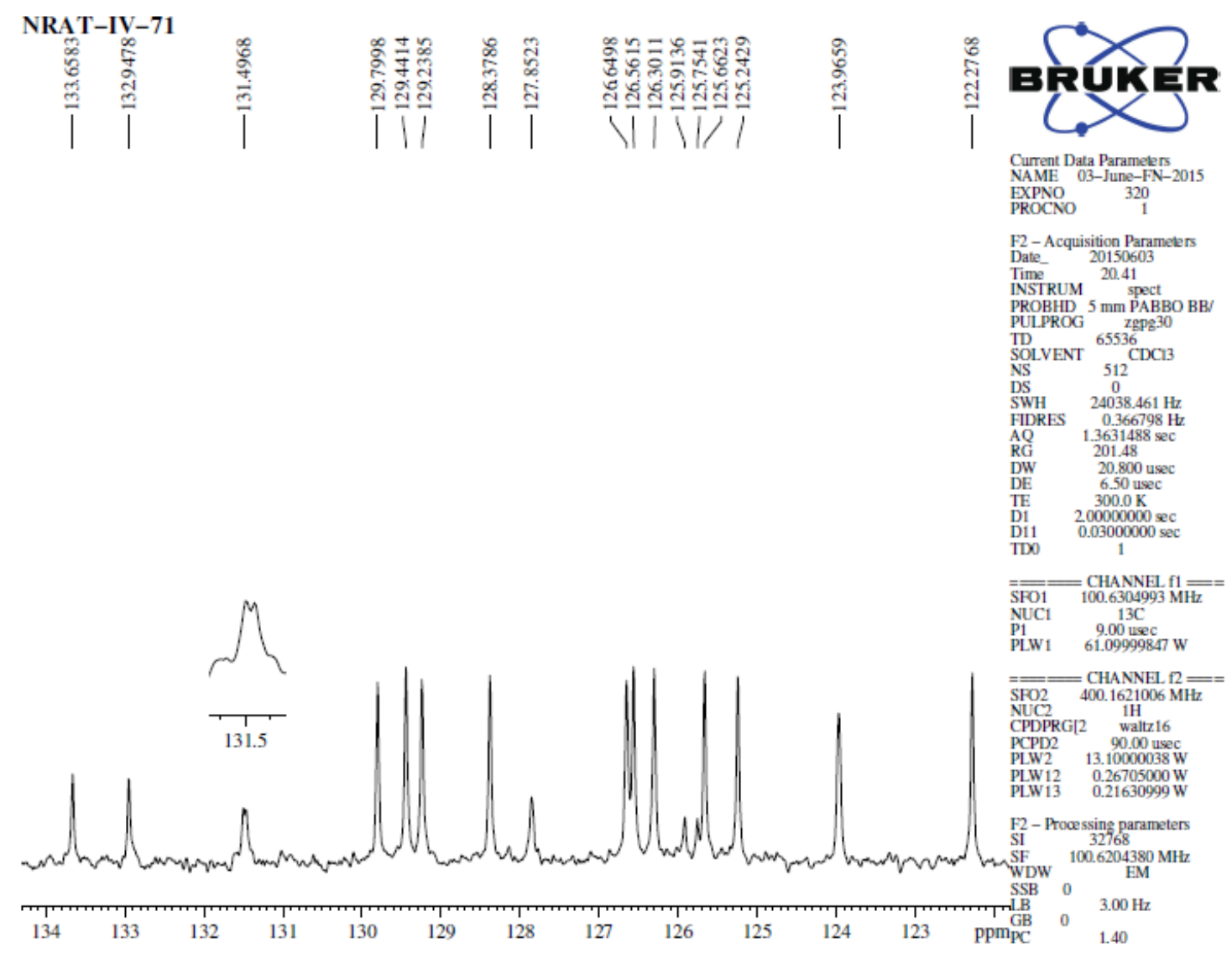

Figure 84: ${ }^{13} \mathrm{C}$ NMR spectrum of $3 \mathrm{j}$ (expansion) 


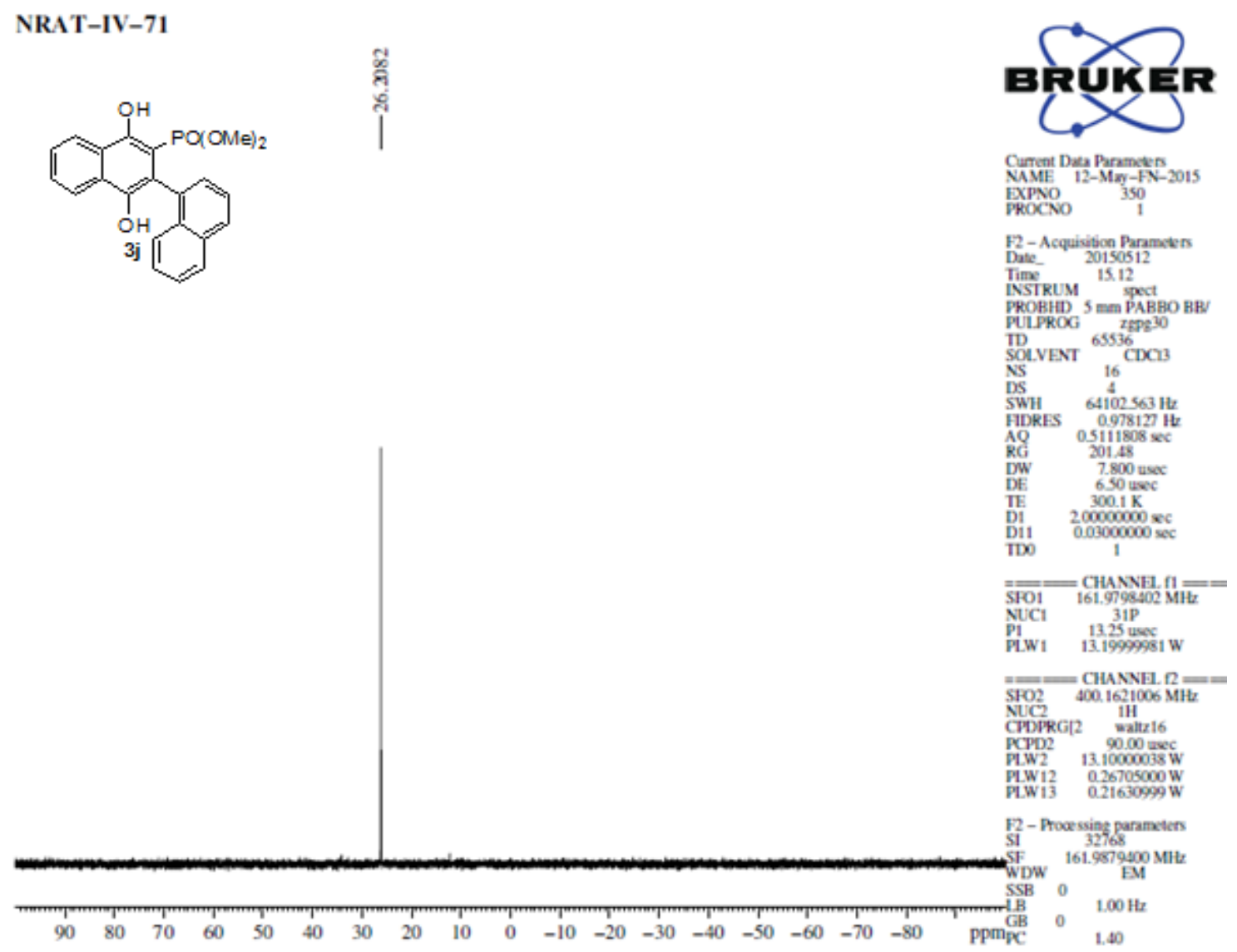

Figure 85: ${ }^{31} \mathrm{P}$ NMR spectrum of $3 \mathrm{j}$

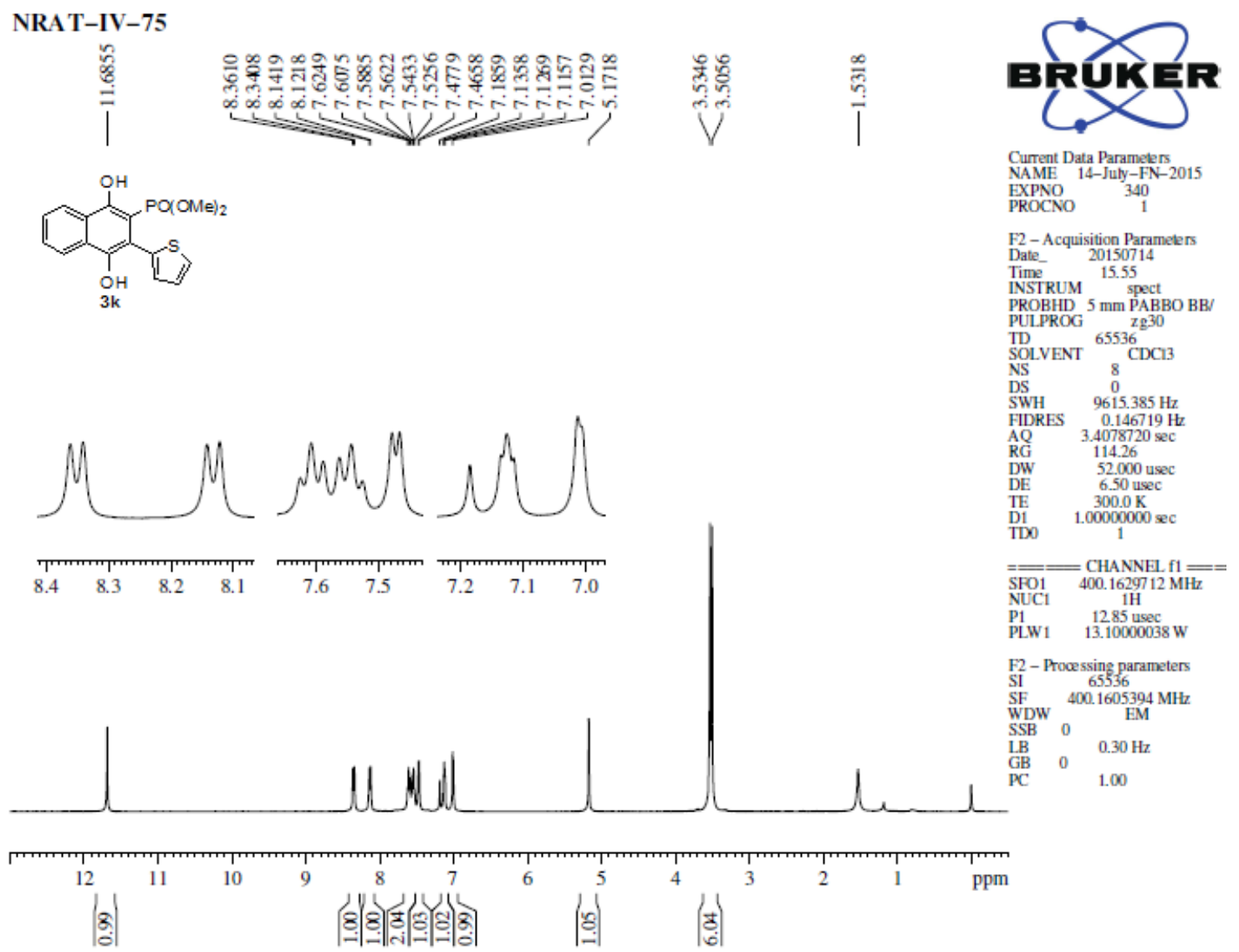

Figure 86: ${ }^{1} \mathrm{H}$ NMR spectrum of $3 \mathrm{k}$ 


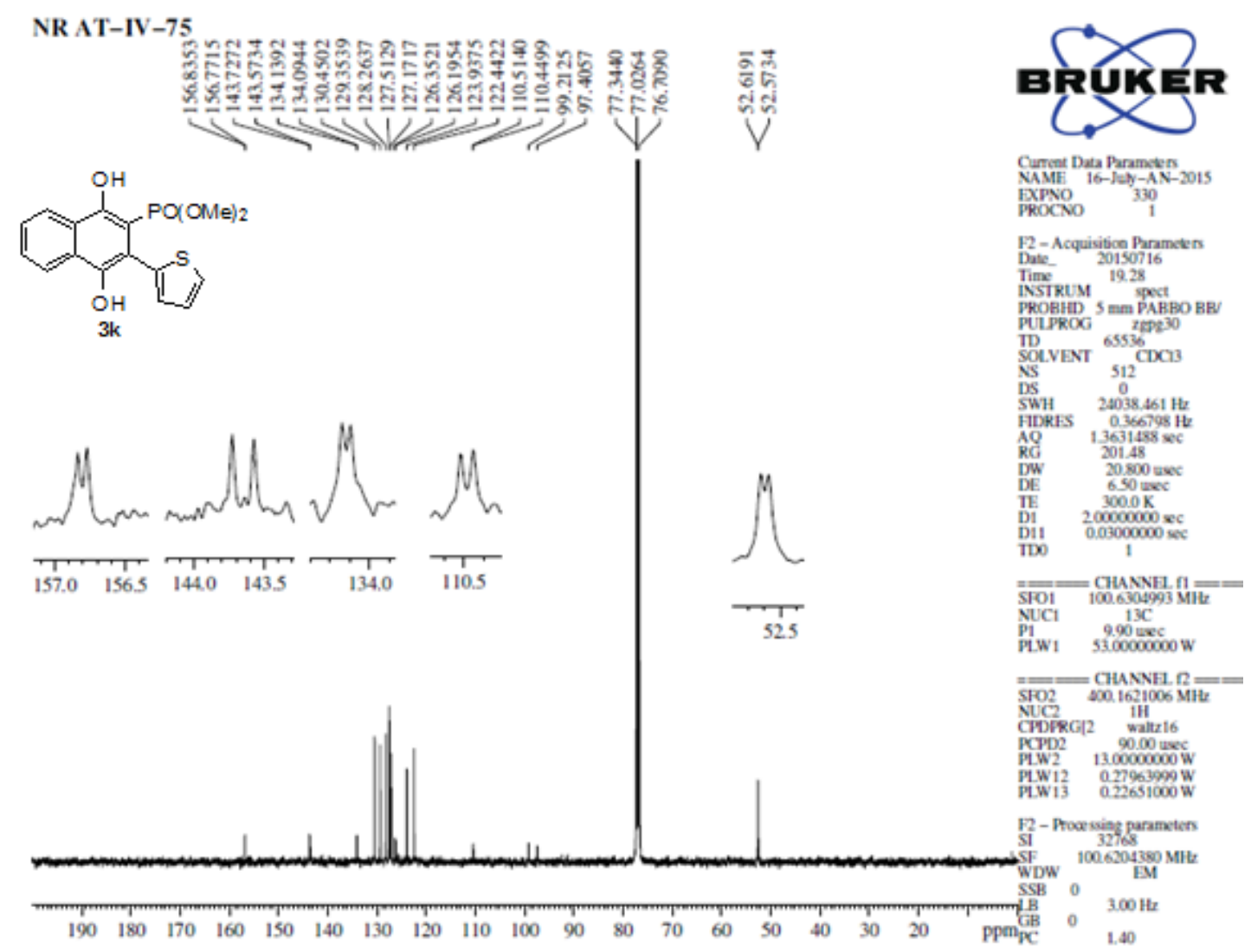

Figure 87: ${ }^{13} \mathrm{C}$ NMR spectrum of $3 k$
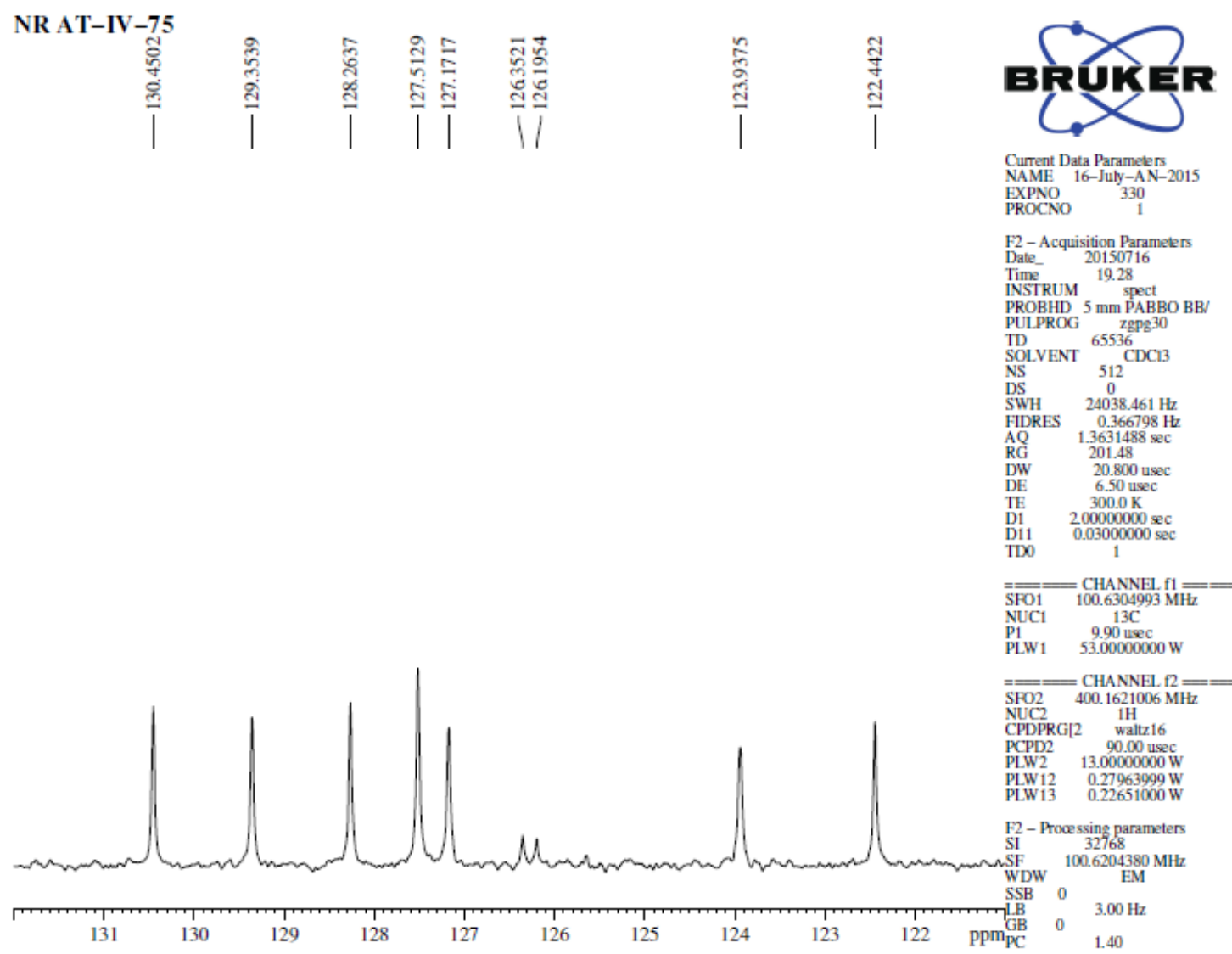

Figure 88: ${ }^{13} \mathrm{C}$ NMR spectrum of $3 \mathrm{k}$ (expansion) 


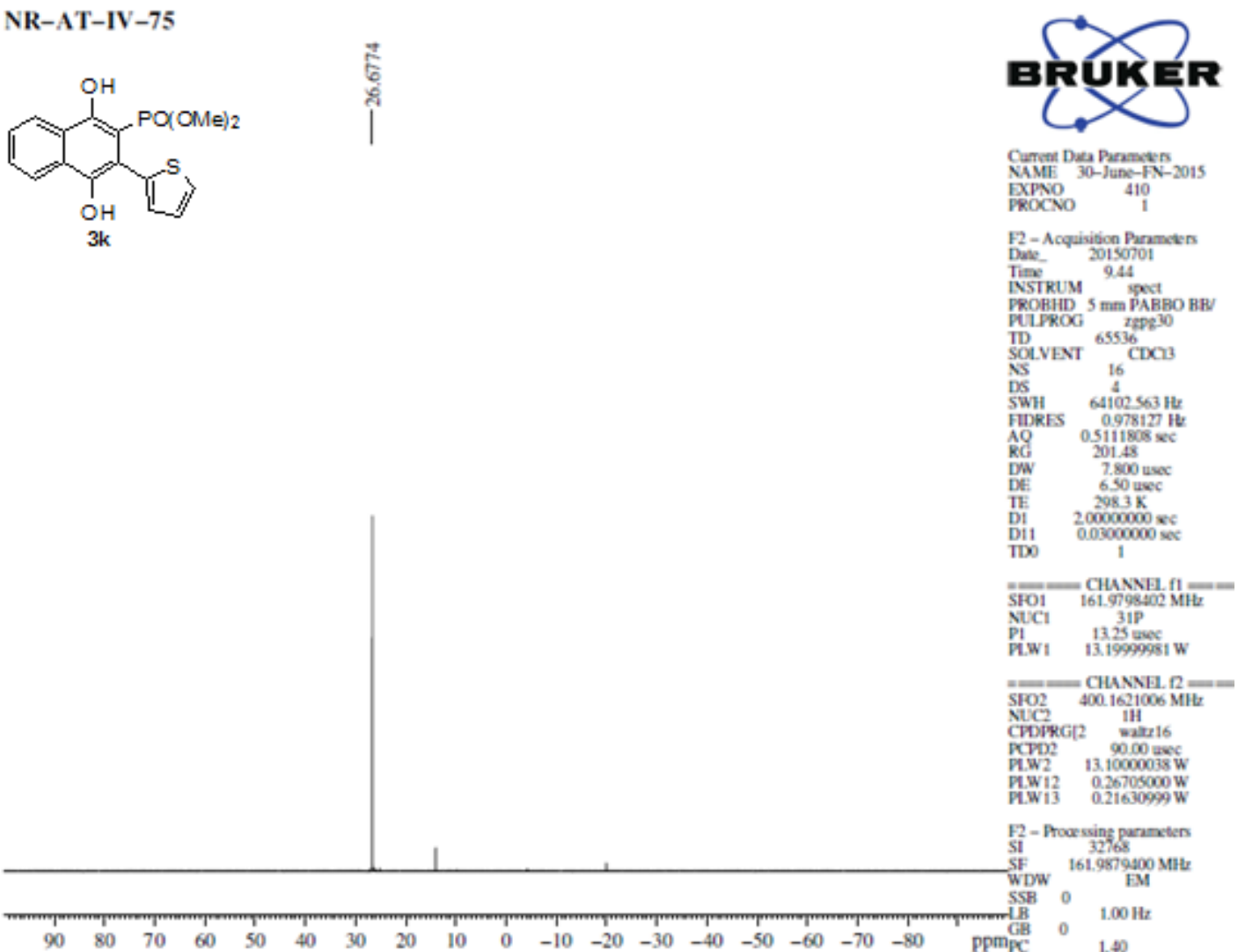

Figure 89: ${ }^{31} \mathrm{P}$ NMR spectrum of $3 \mathrm{k}$

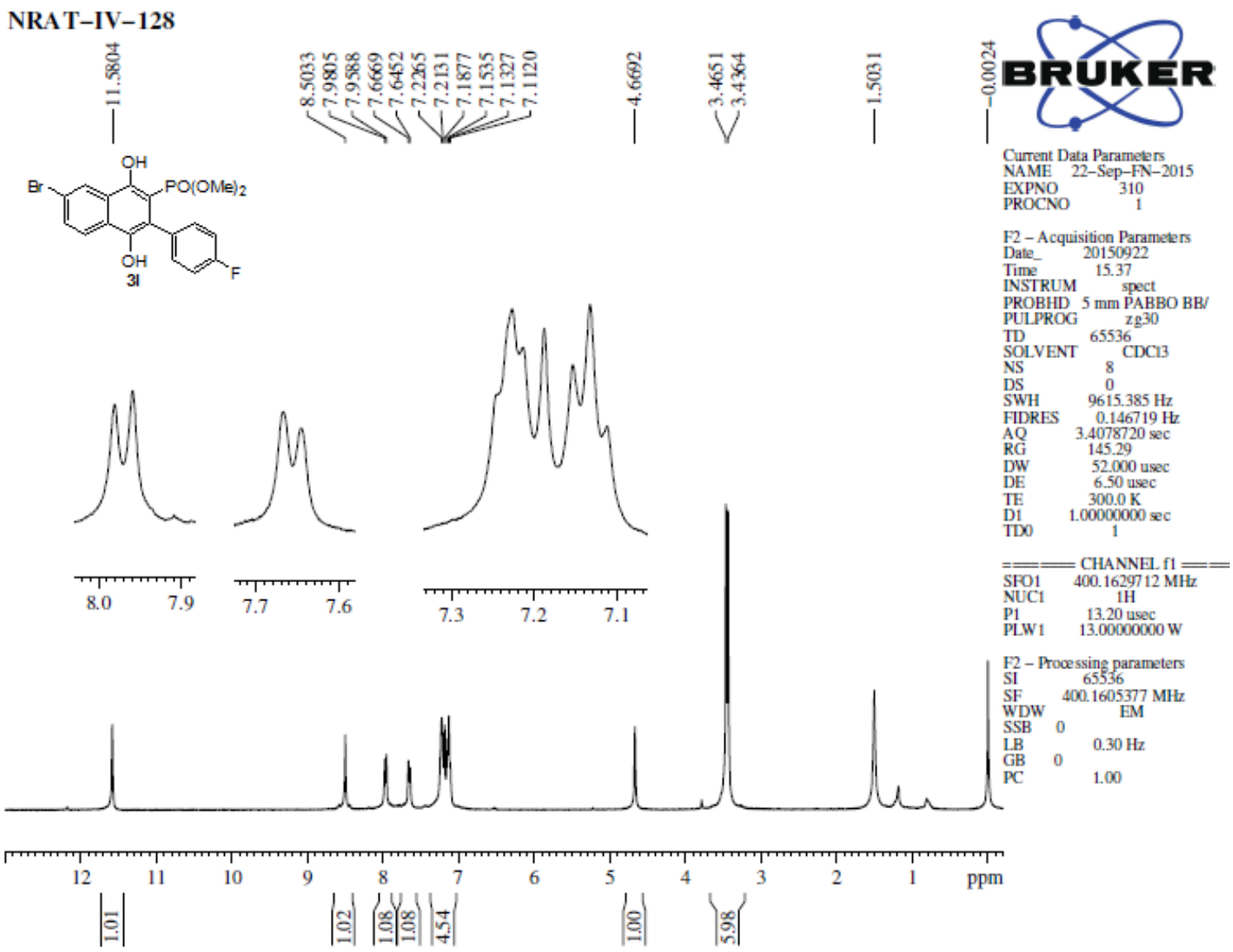

Figure 90: ${ }^{1} \mathrm{H}$ NMR spectrum of 31 


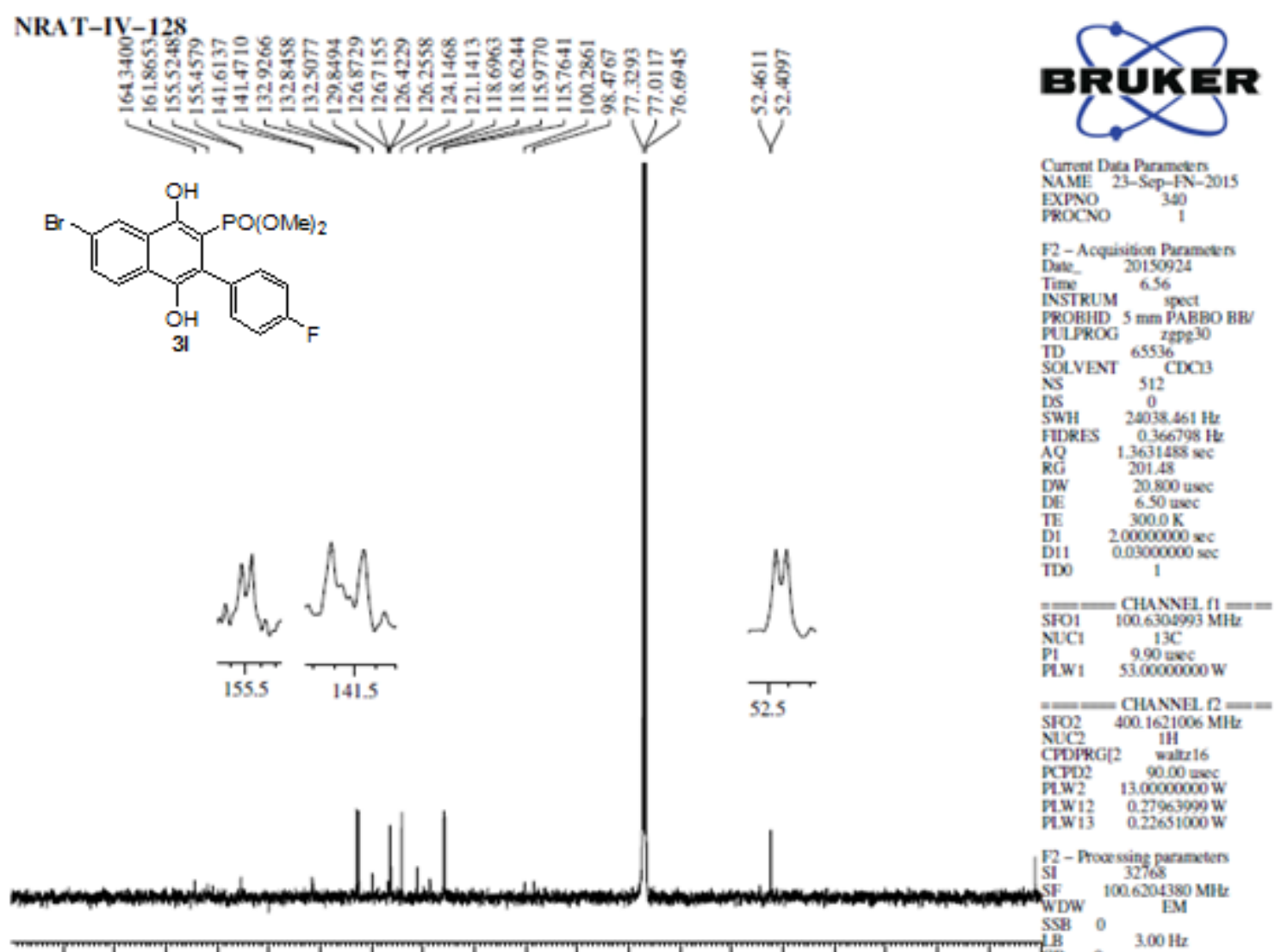

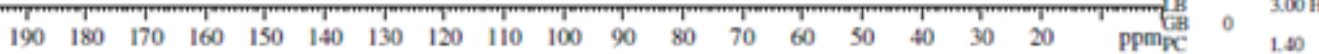

Figure 91: ${ }^{13} \mathrm{C}$ NMR spectrum of 31

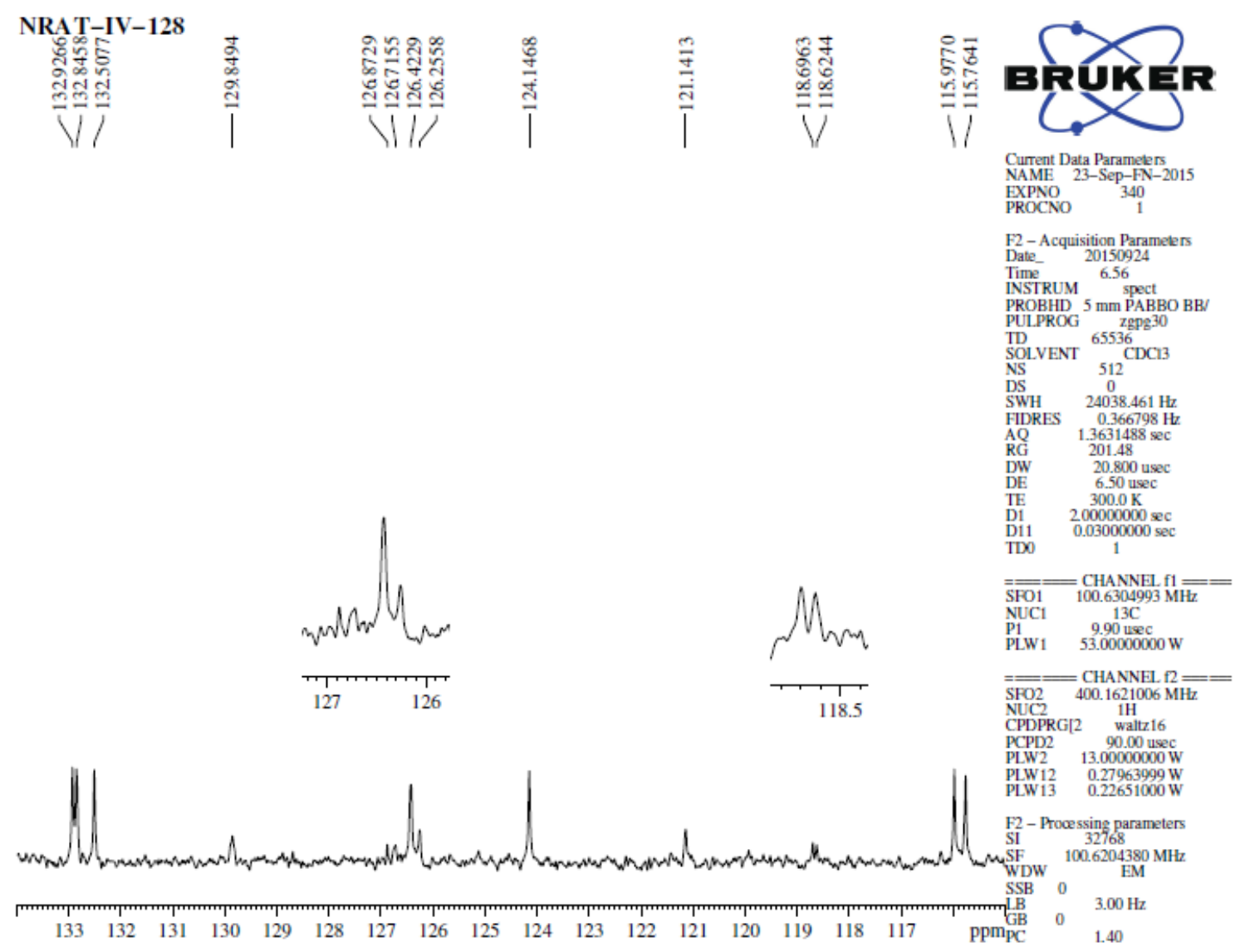

Figure 92: ${ }^{13} \mathrm{C}$ NMR spectrum of 31 (expansion) 

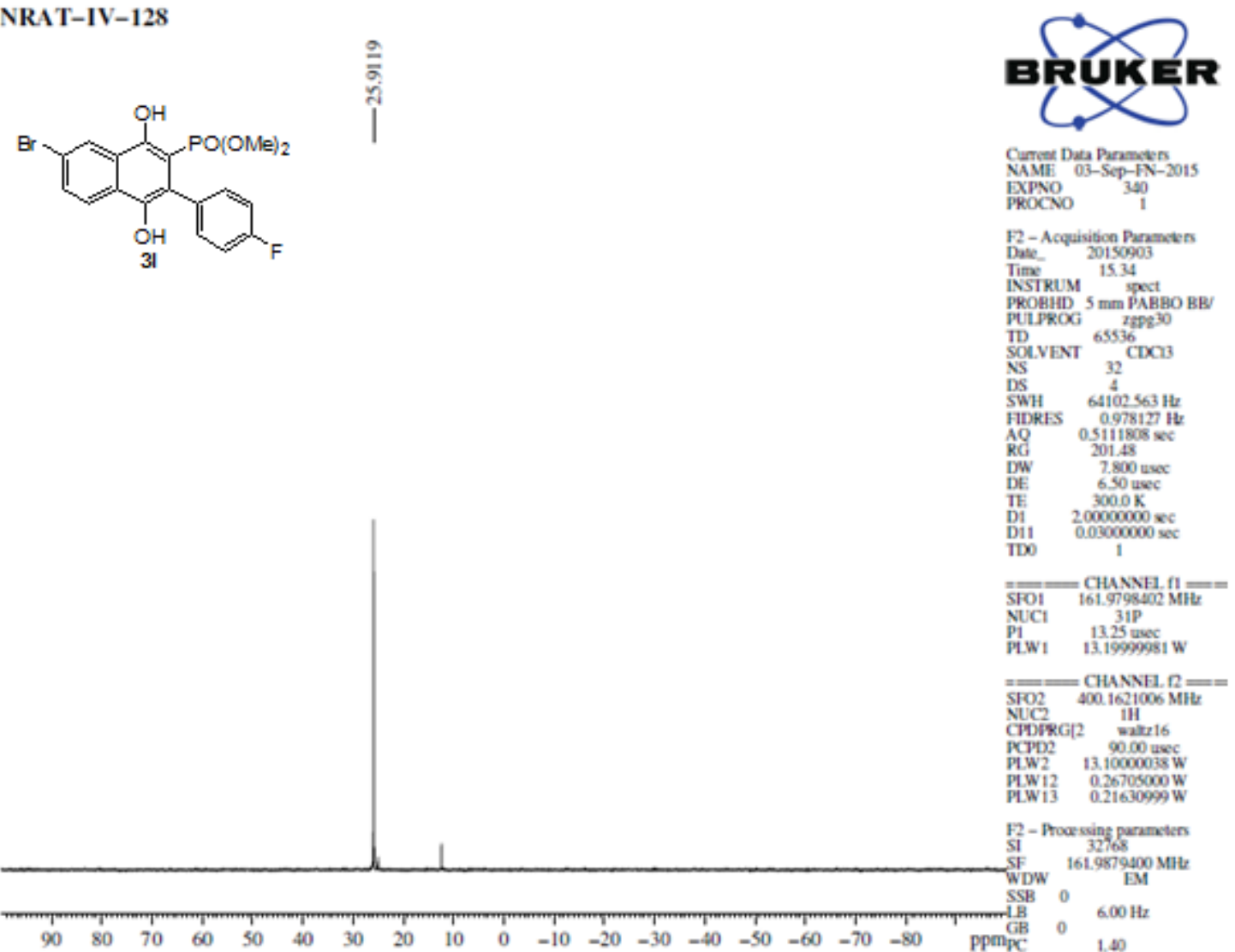

Figure 93: ${ }^{31} \mathrm{P}$ NMR spectrum of 31

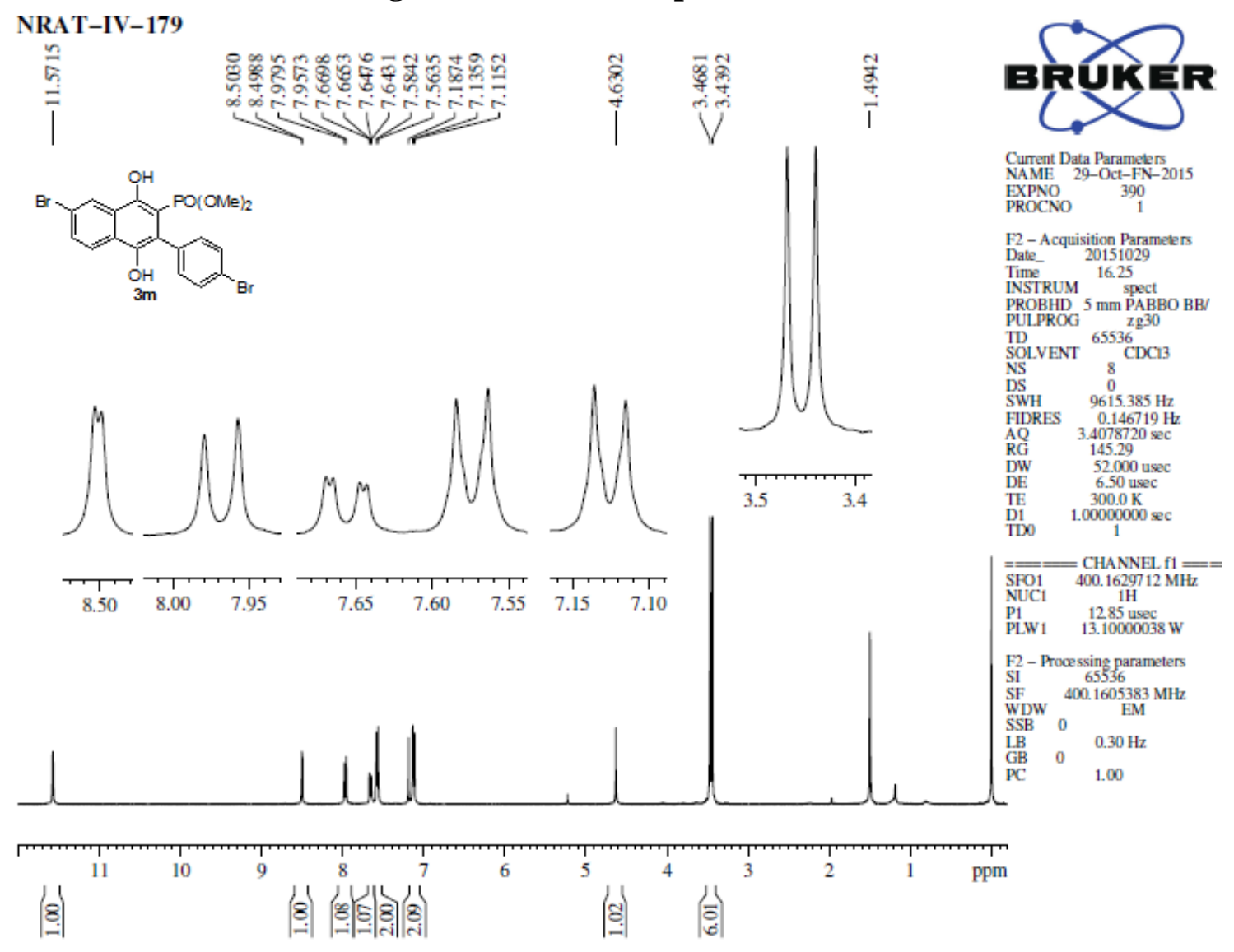

Figure 94: ${ }^{1} \mathrm{H}$ NMR spectrum of $3 \mathrm{~m}$ 


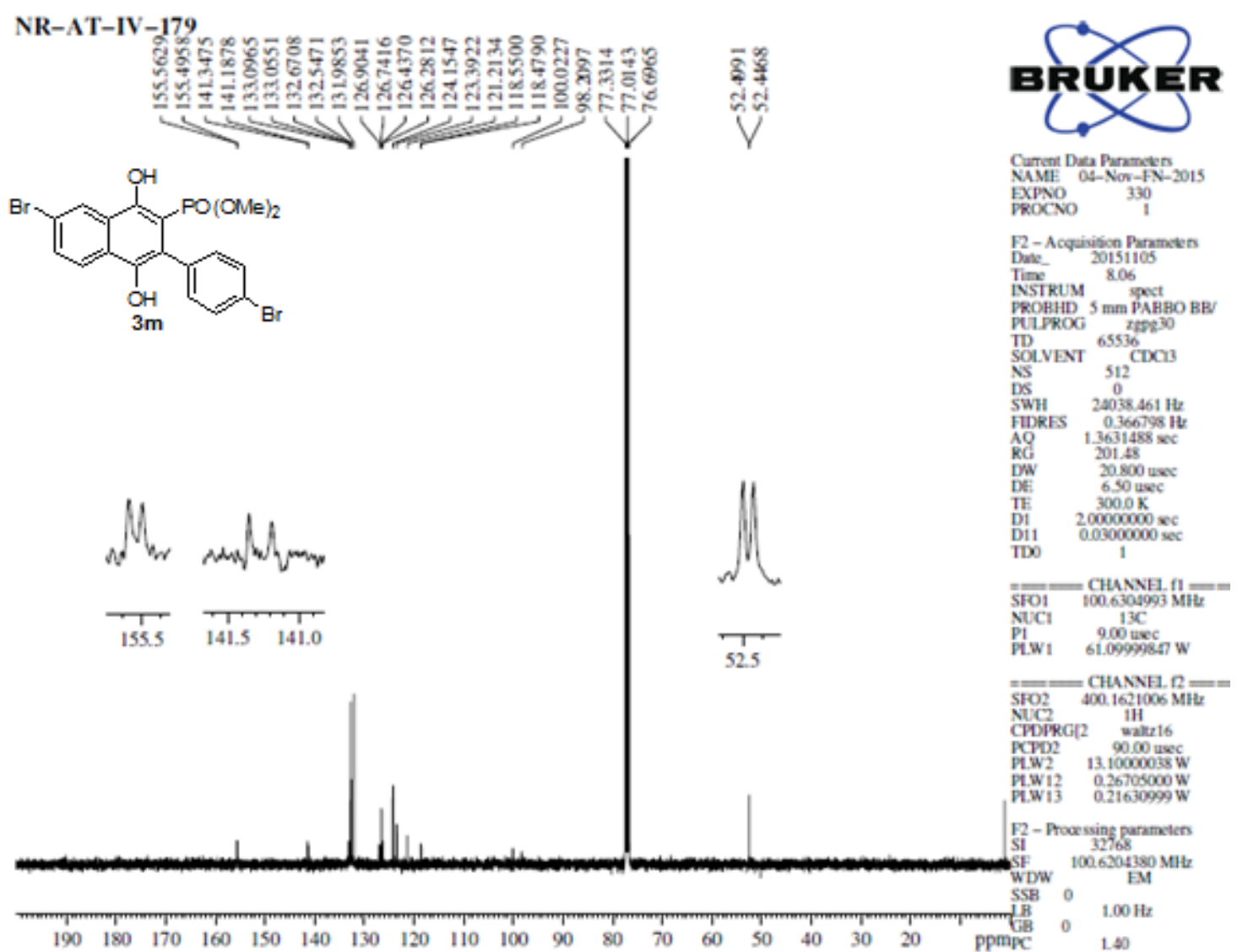

Figure 95: ${ }^{13} \mathrm{C}$ NMR spectrum of $3 \mathrm{~m}$

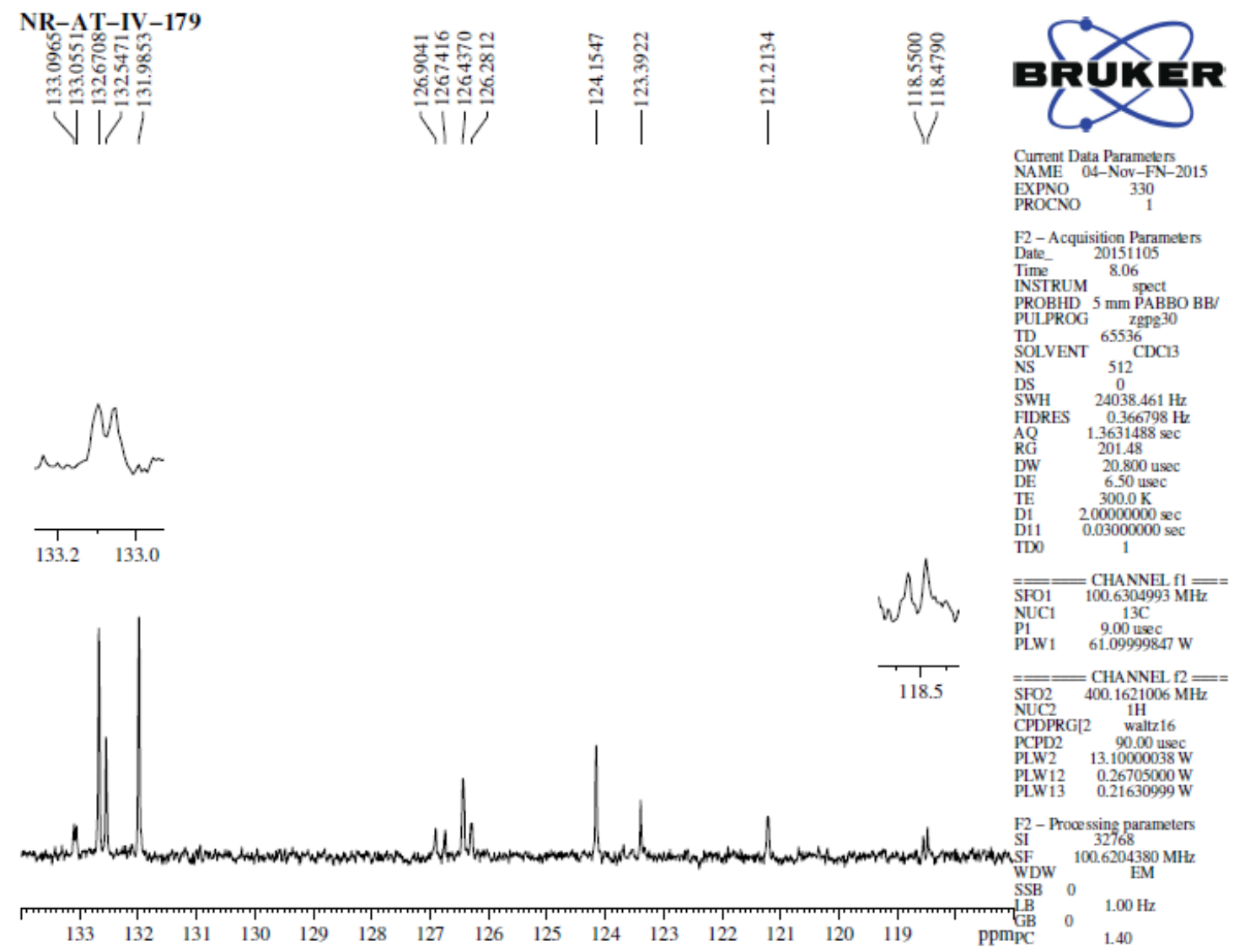

Figure 96: ${ }^{13} \mathrm{C}$ NMR spectrum of $3 \mathrm{~m}$ (expansion) 


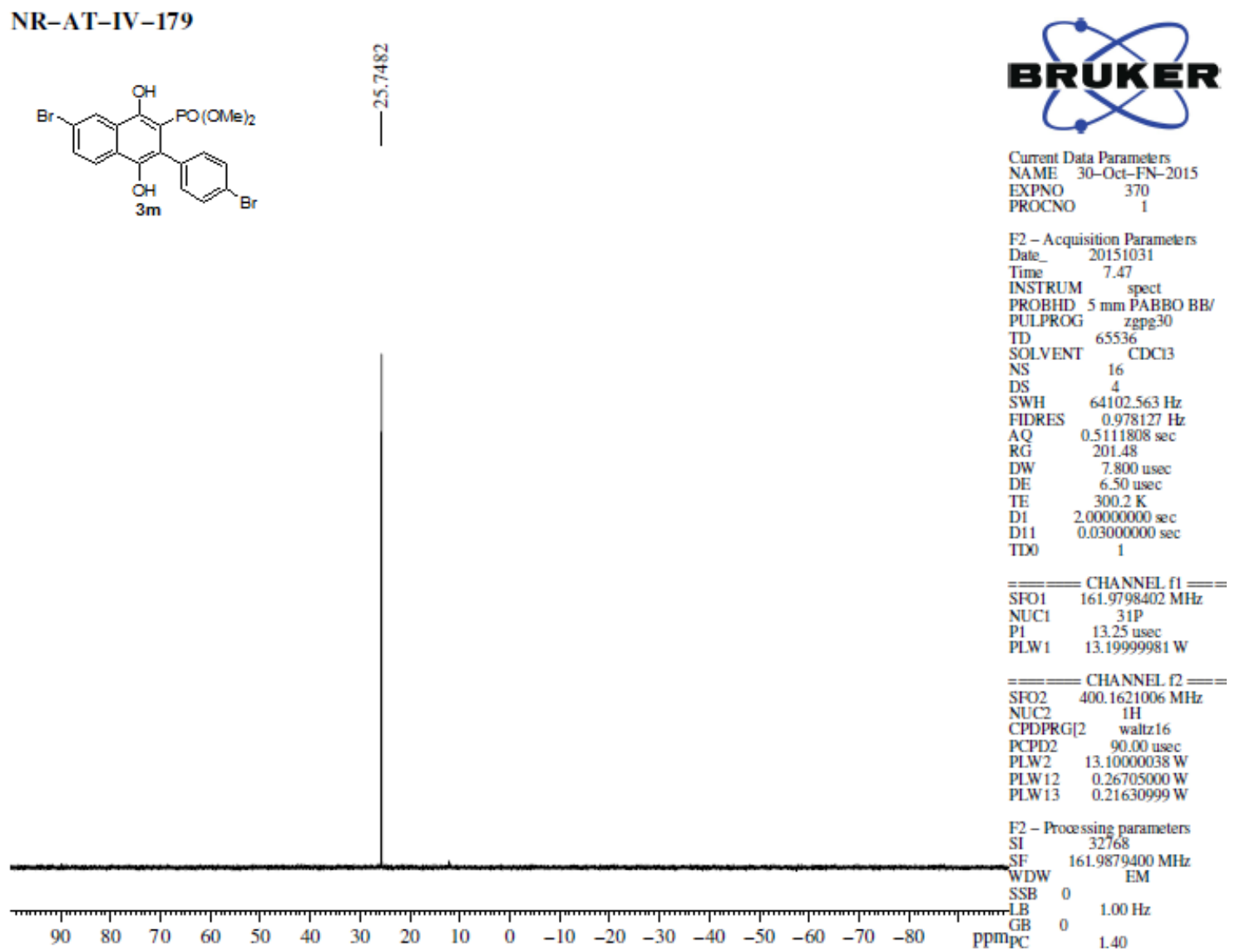

Figure 97: ${ }^{31} \mathrm{P}$ NMR spectrum of $3 \mathrm{~m}$

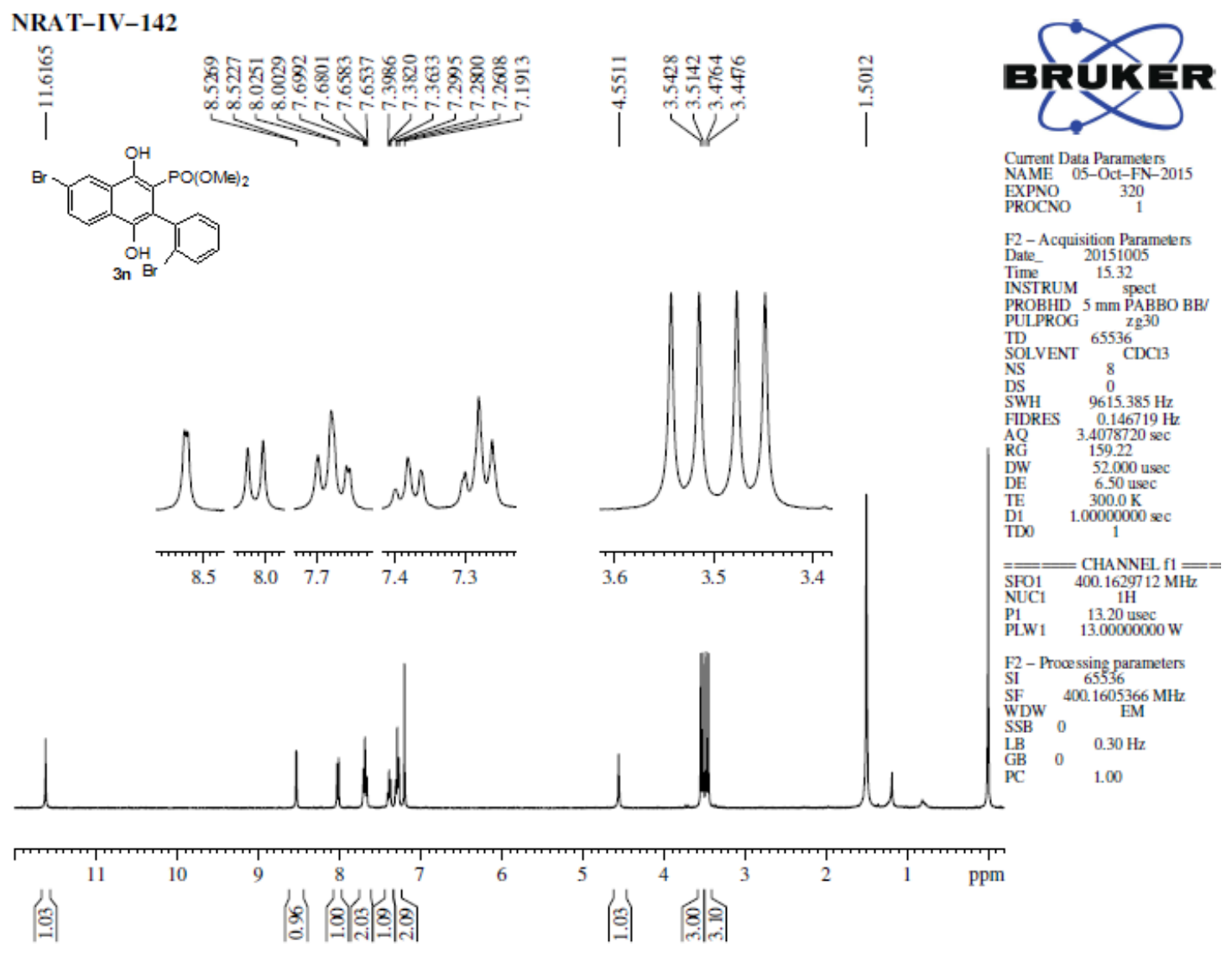

Figure 98: ${ }^{1} \mathrm{H}$ NMR spectrum of $3 n$ 


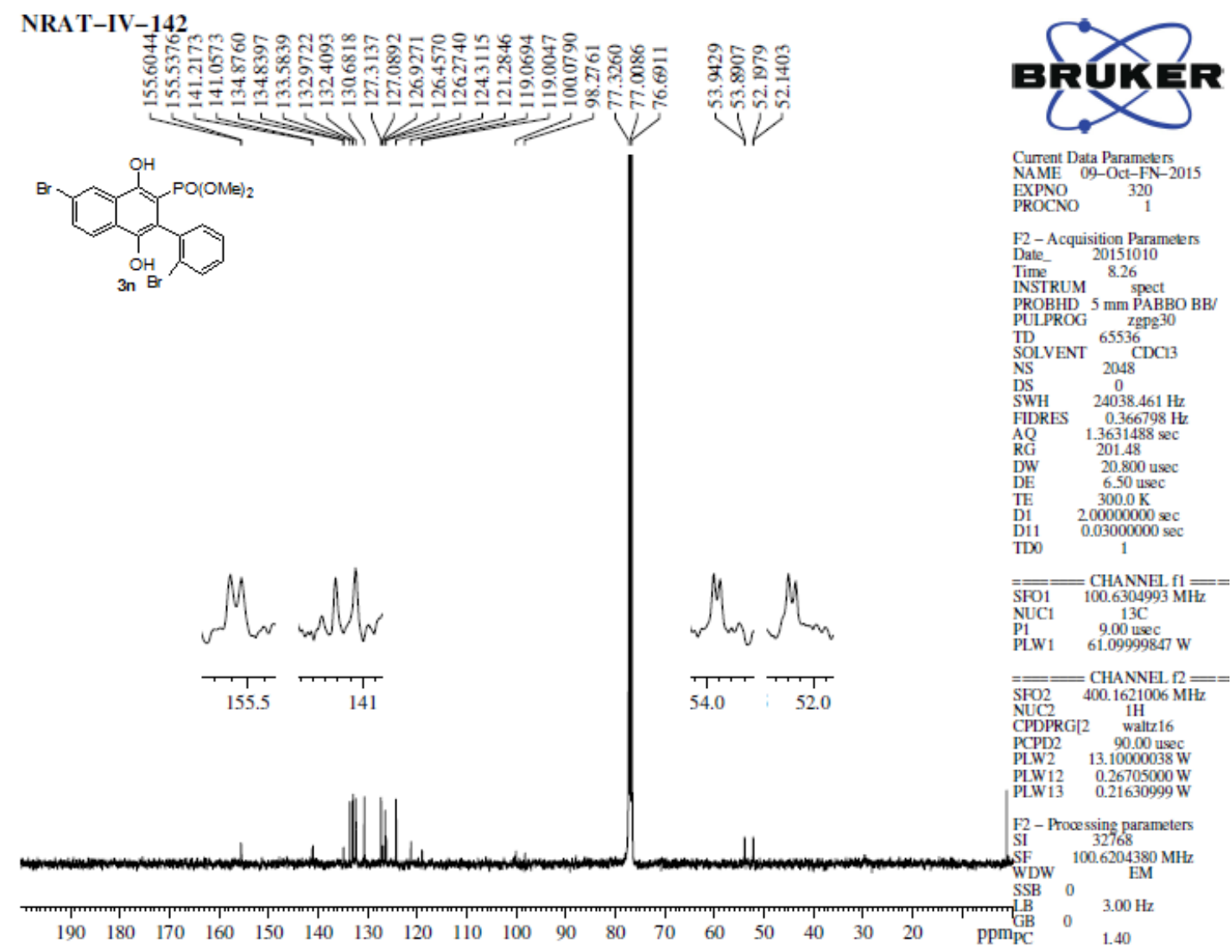

Figure 99: ${ }^{13} \mathrm{C}$ NMR spectrum of $3 n$

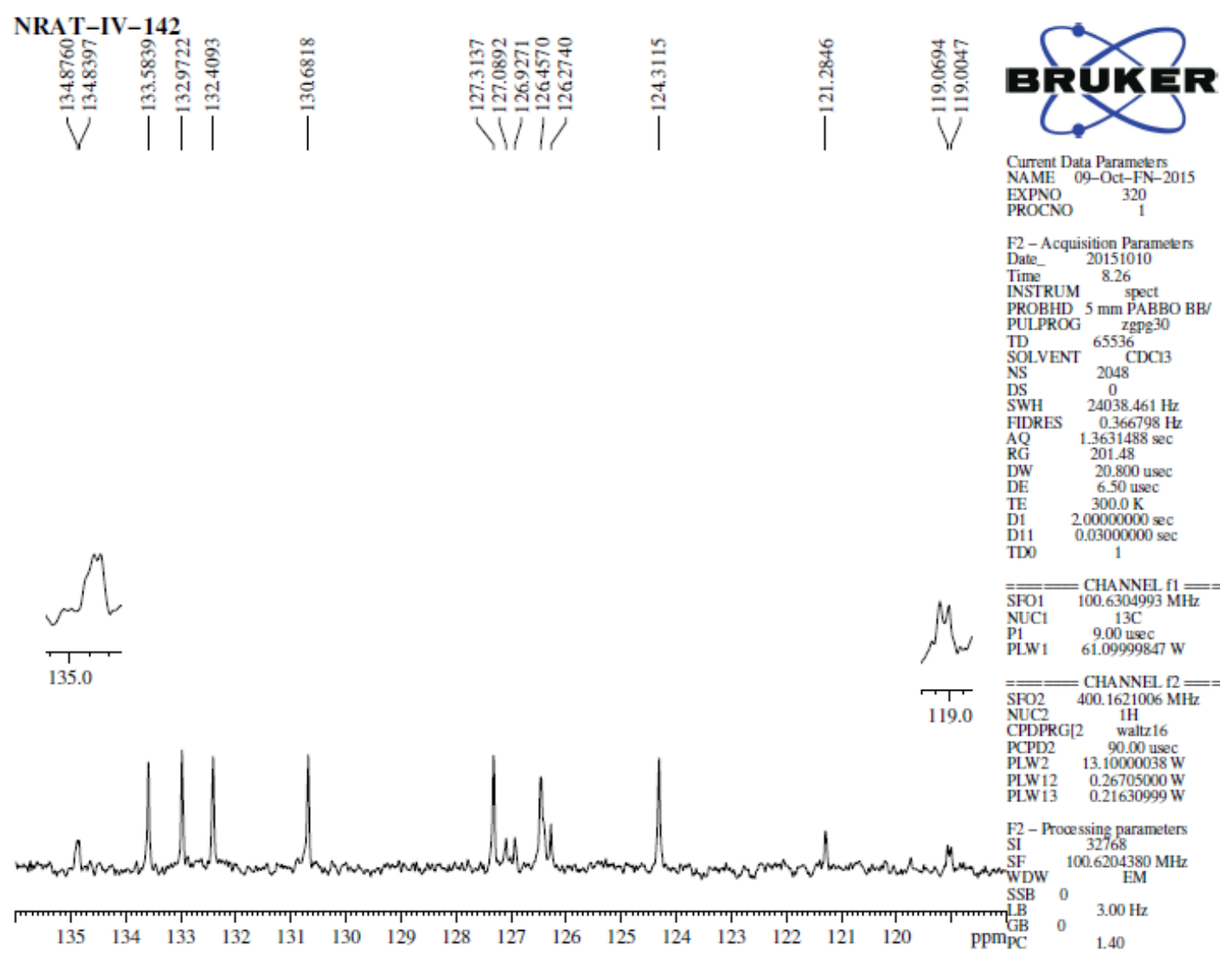

Figure 100: ${ }^{13} \mathrm{C}$ NMR spectrum of $3 \mathrm{n}$ (expansion) 


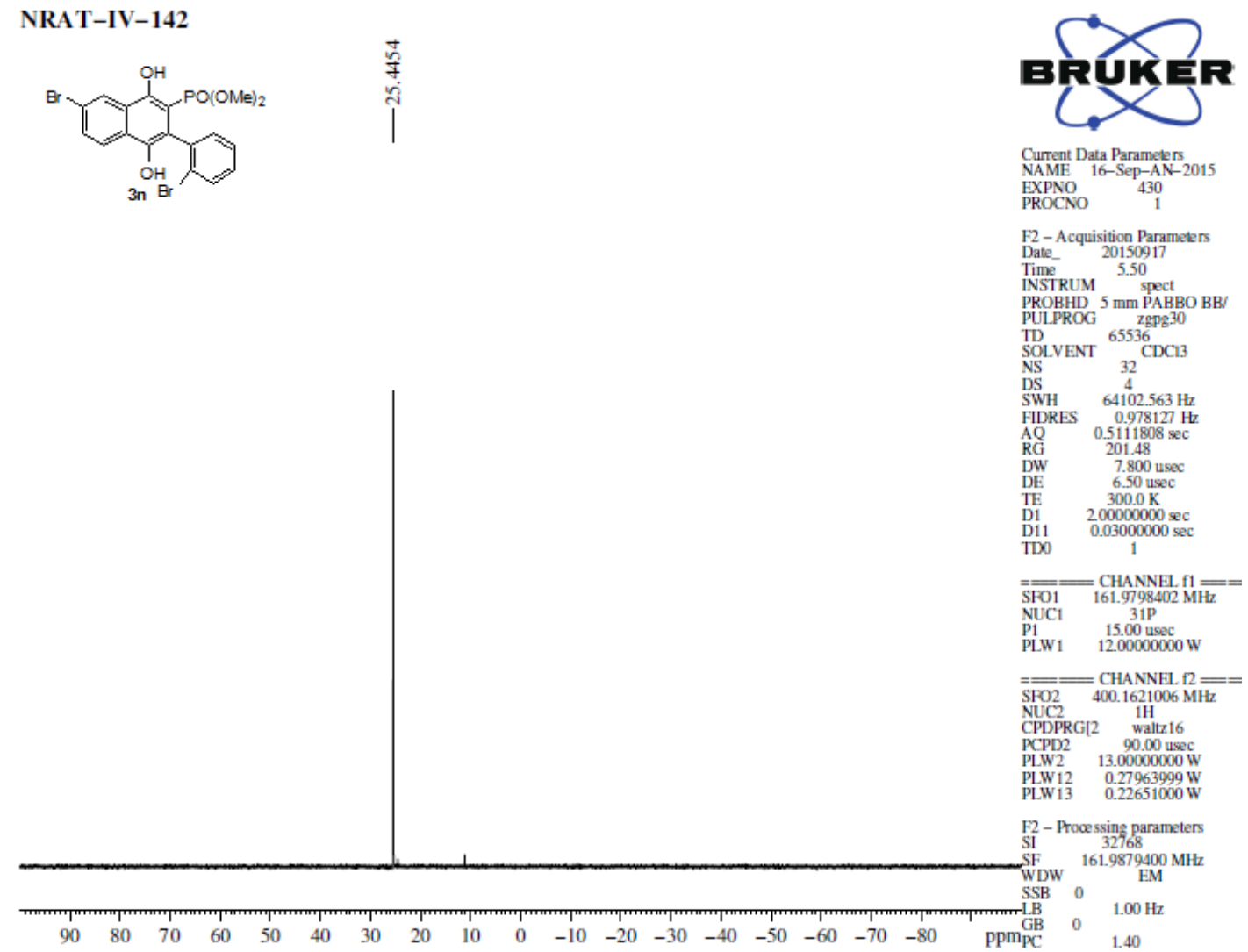

Figure 101: ${ }^{31} \mathrm{P}$ NMR spectrum of $3 n$

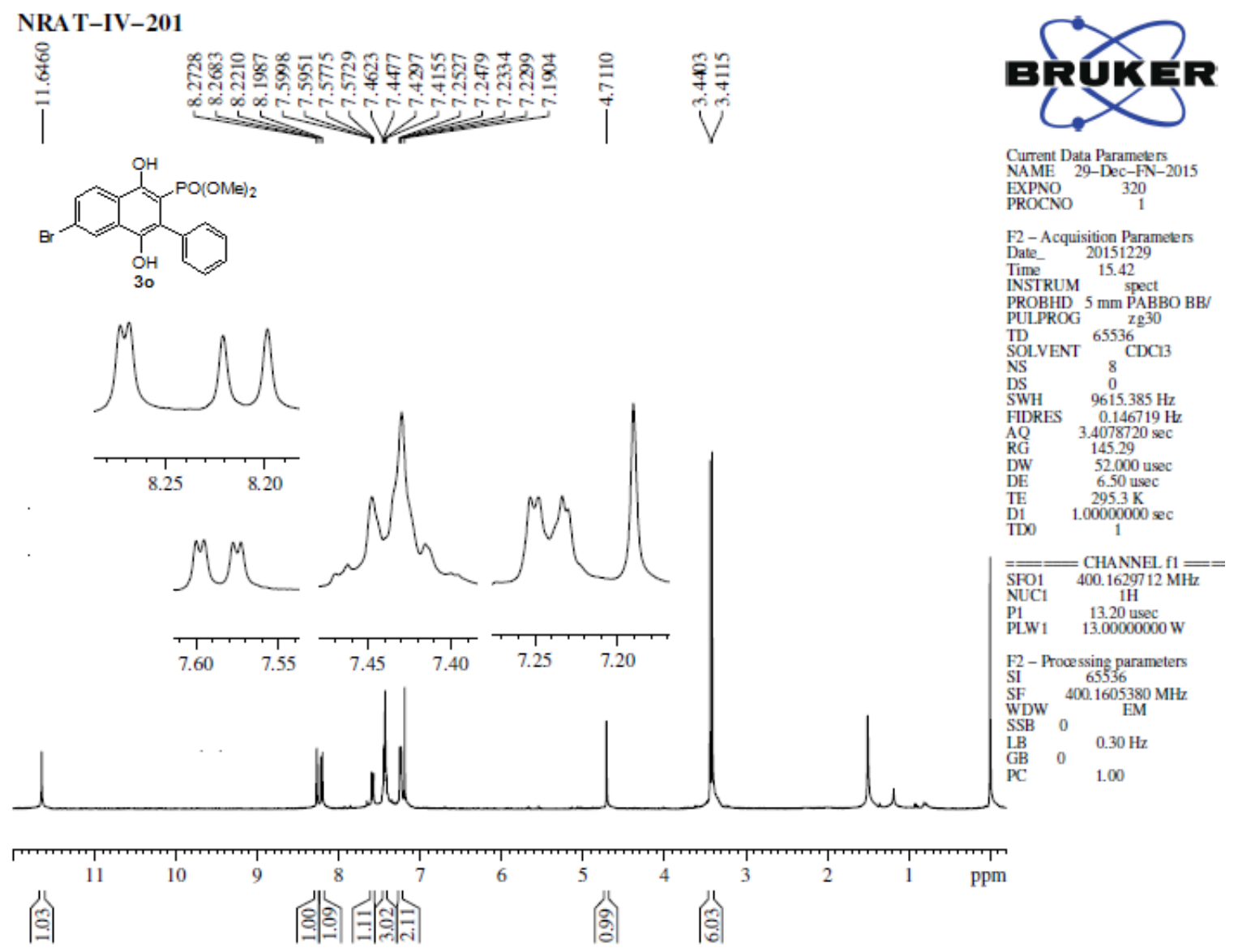

Figure 102: ${ }^{1} \mathrm{H}$ NMR spectrum of 30 


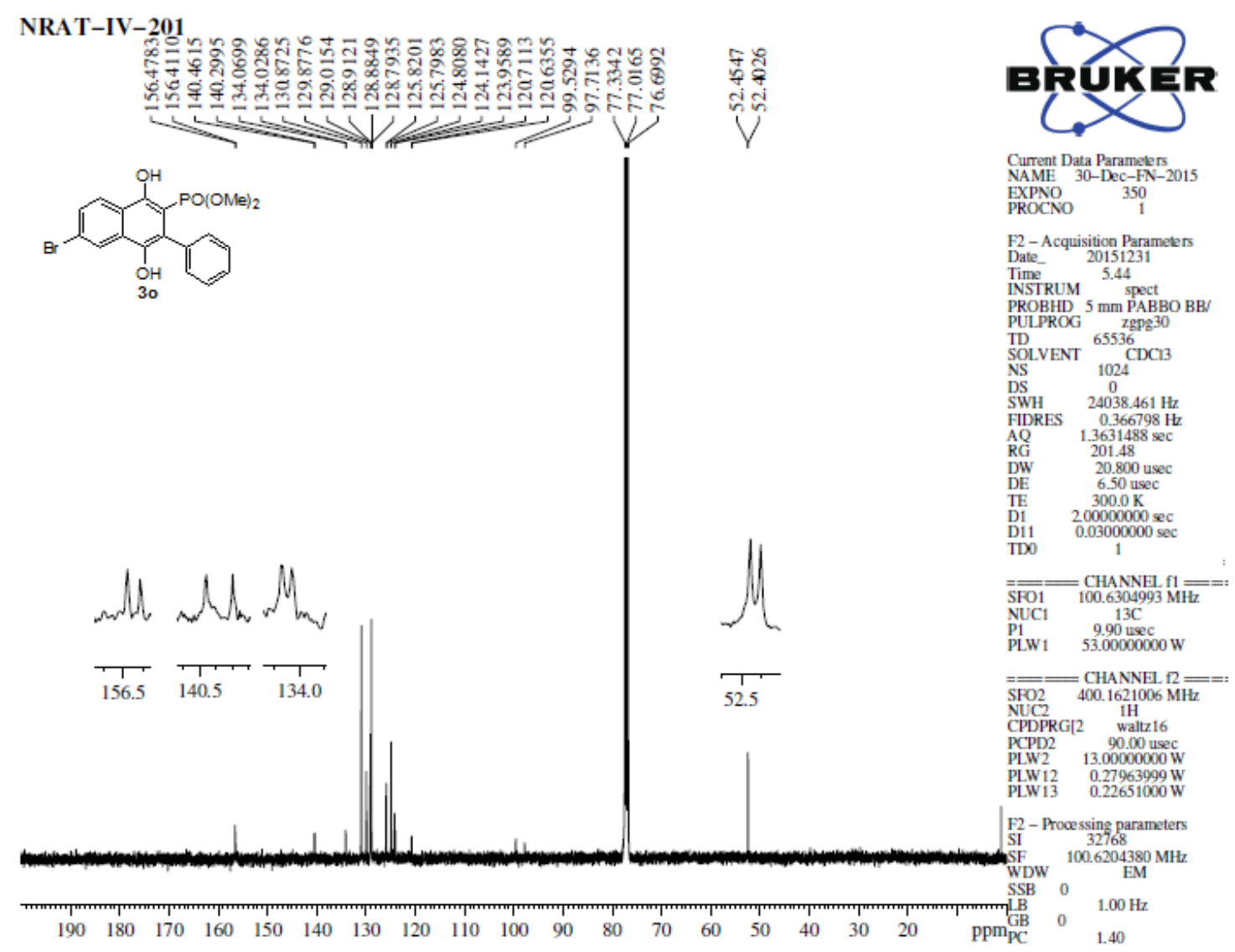

Figure 103: ${ }^{13} \mathrm{C}$ NMR spectrum of 30

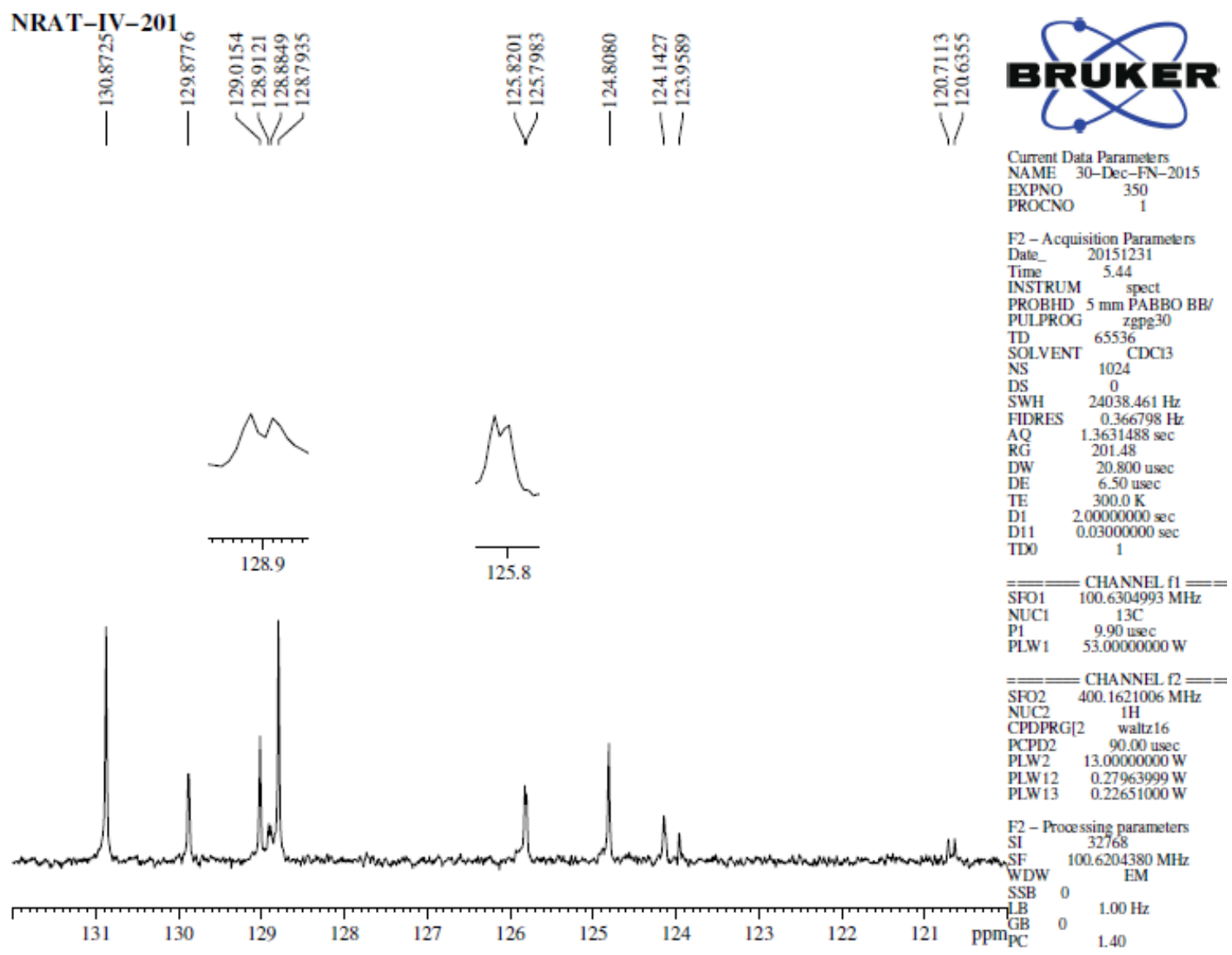

Figure 104: ${ }^{13} \mathrm{C}$ NMR spectrum of 30 (expansion) 


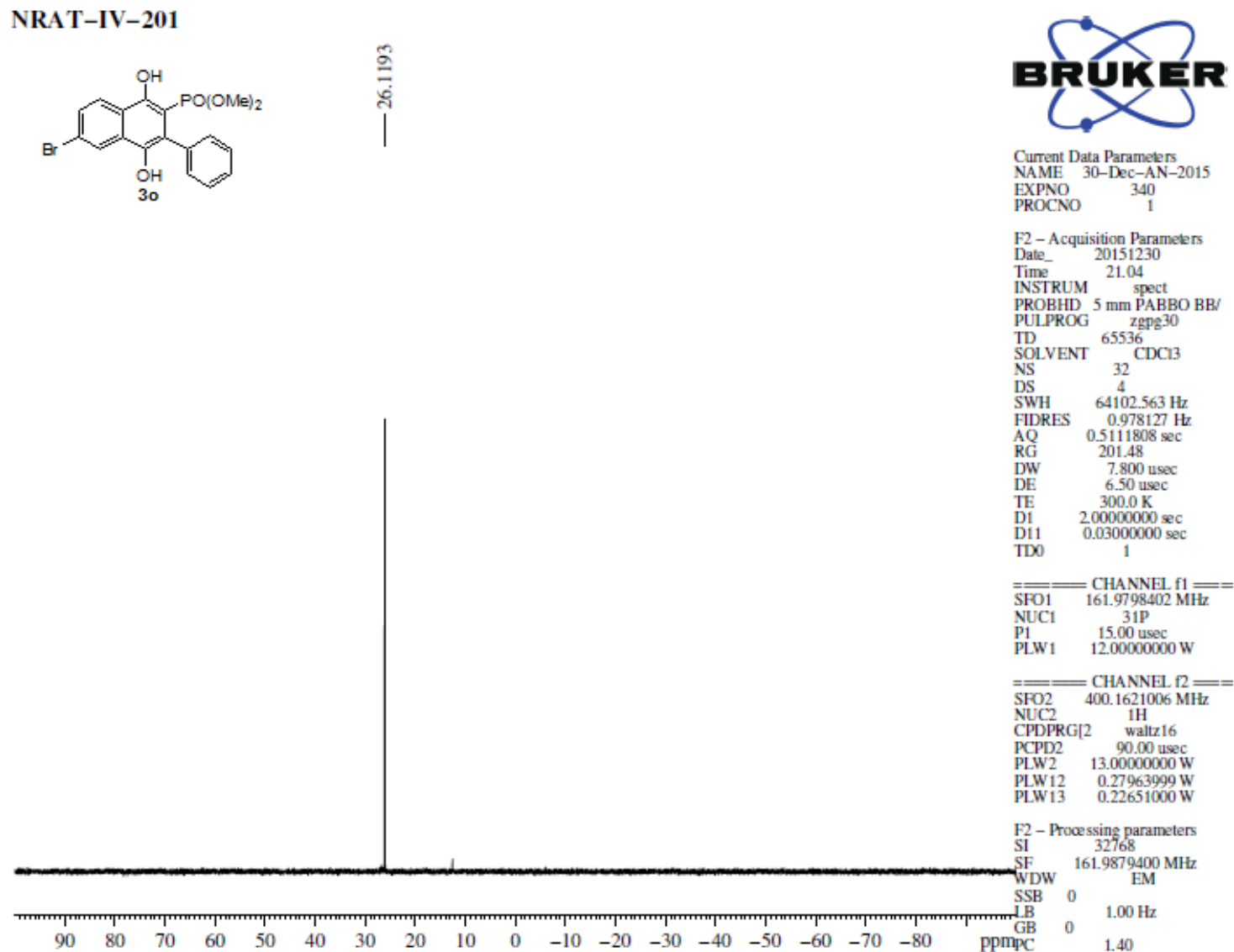

Figure 105: ${ }^{31} \mathrm{P}$ NMR spectrum of 30

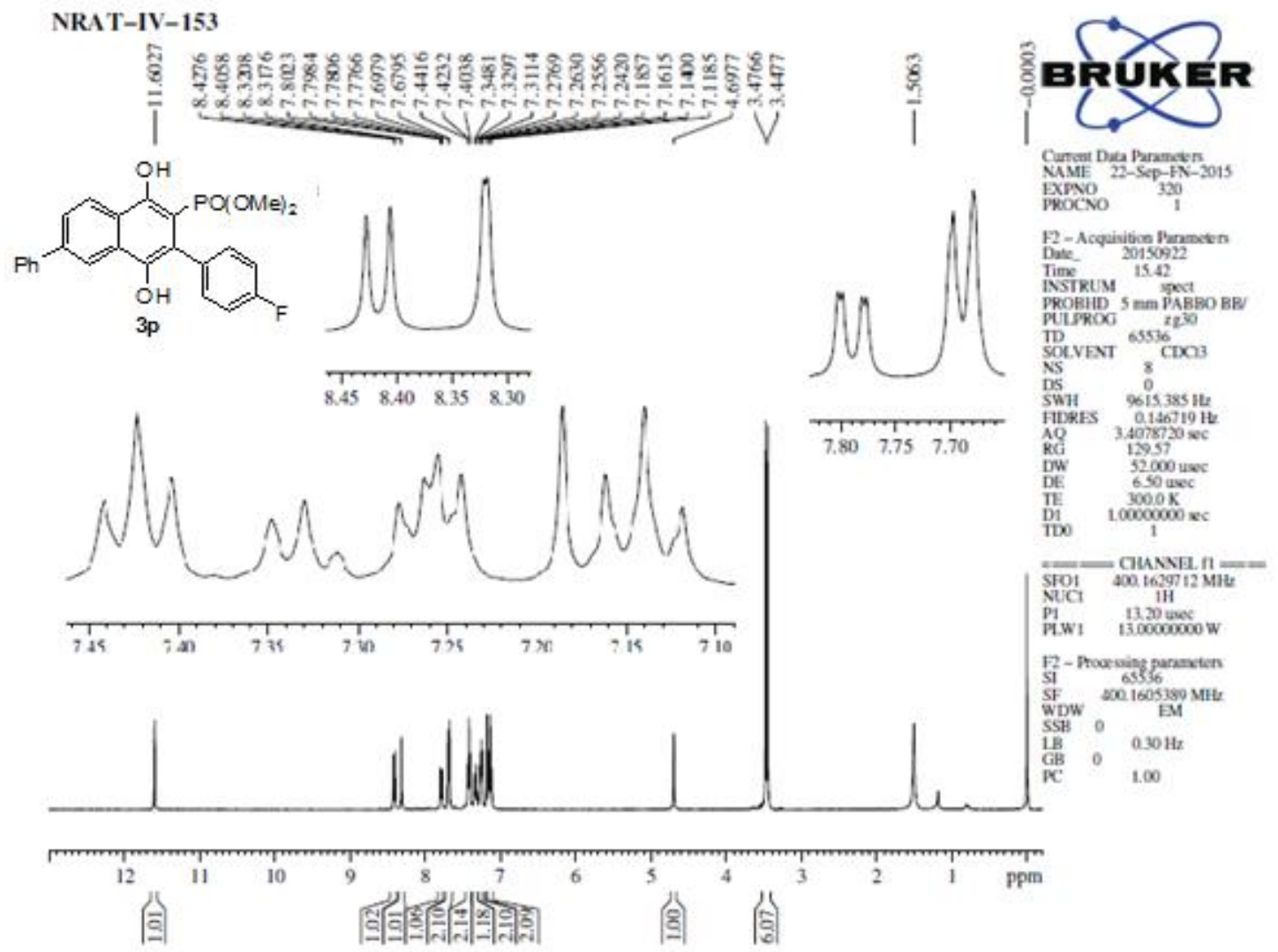

Figure 106: ${ }^{1} \mathrm{H}$ NMR spectrum of $3 p$ 


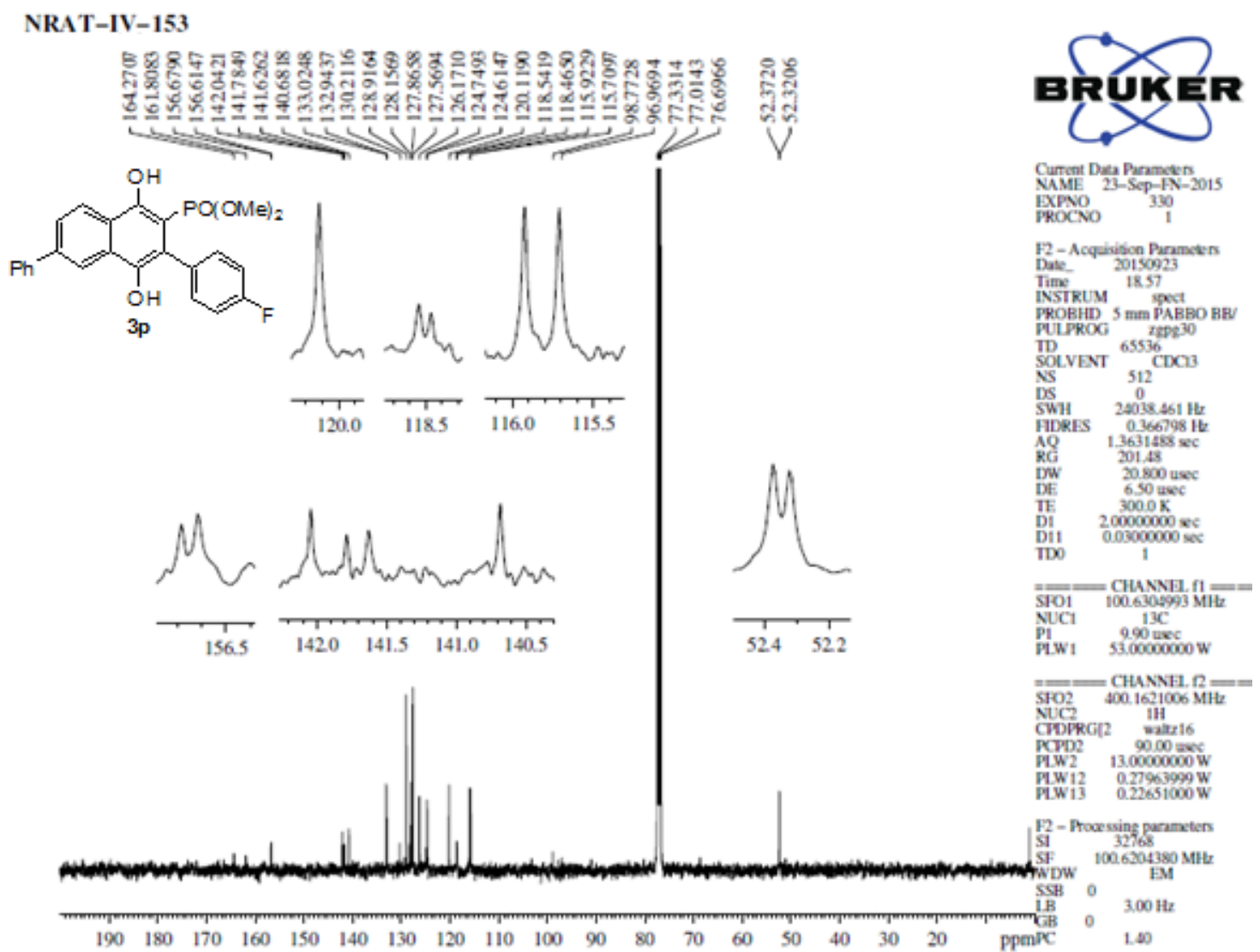

Figure 107: ${ }^{13} \mathrm{C}$ NMR spectrum of $3 p$

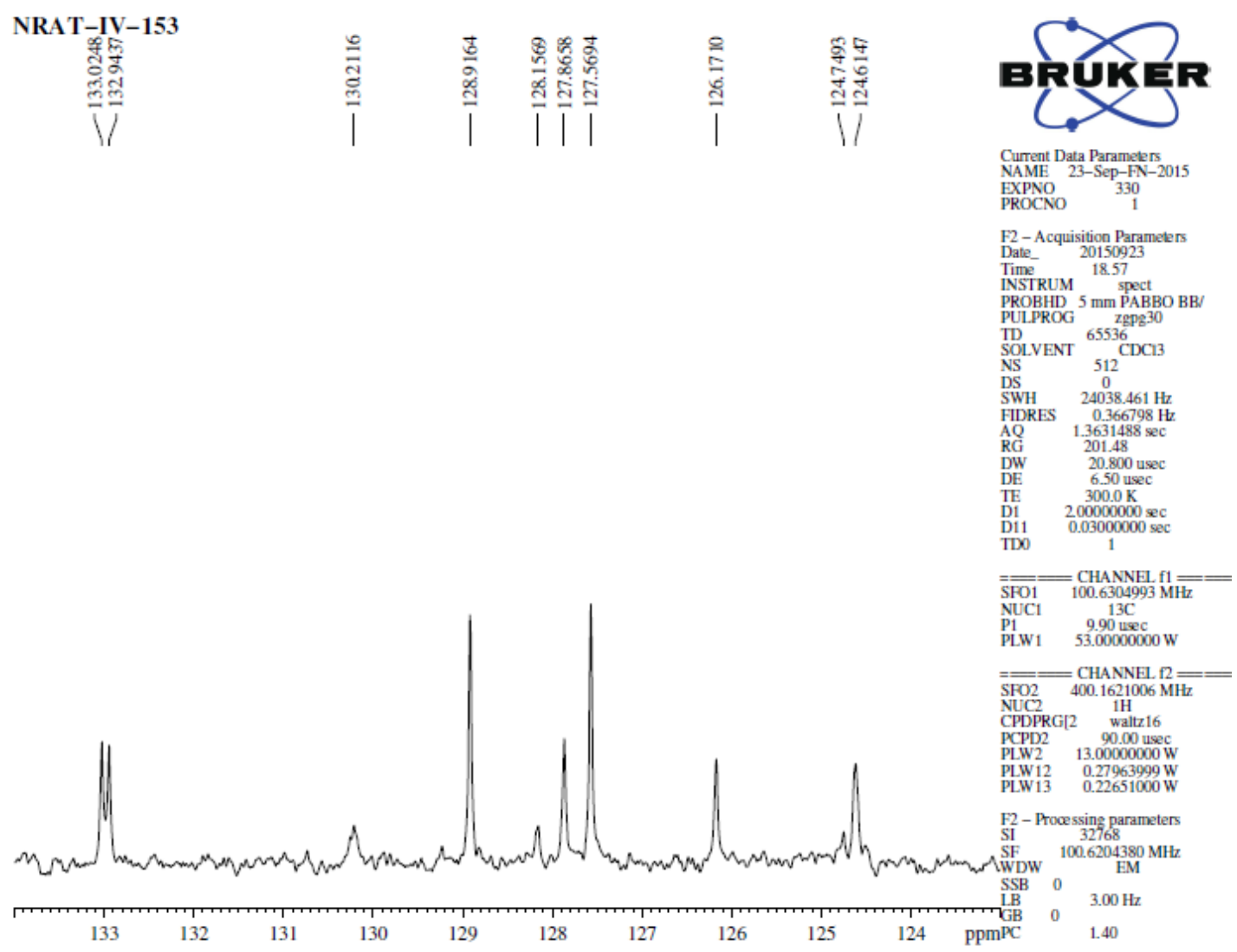

Figure 108: ${ }^{13} \mathrm{C}$ NMR spectrum of $3 p$ (expansion) 
NR-AT-IV-153<smiles>COc1c(-c2ccc(F)cc2)c(O)c2cc(-c3ccccc3)ccc2c1O</smiles>

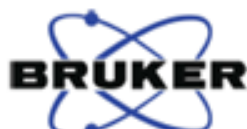

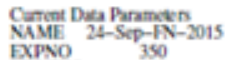

EXNo
WROCN

F2-Acquisitice Prameses

Time

INSTRUM sedt

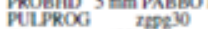

T 655360

SOLVENT ${ }_{16}{ }^{\mathrm{CDOO}}$

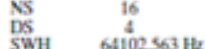

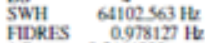

$100 \operatorname{sinsos} \mathrm{sec}$

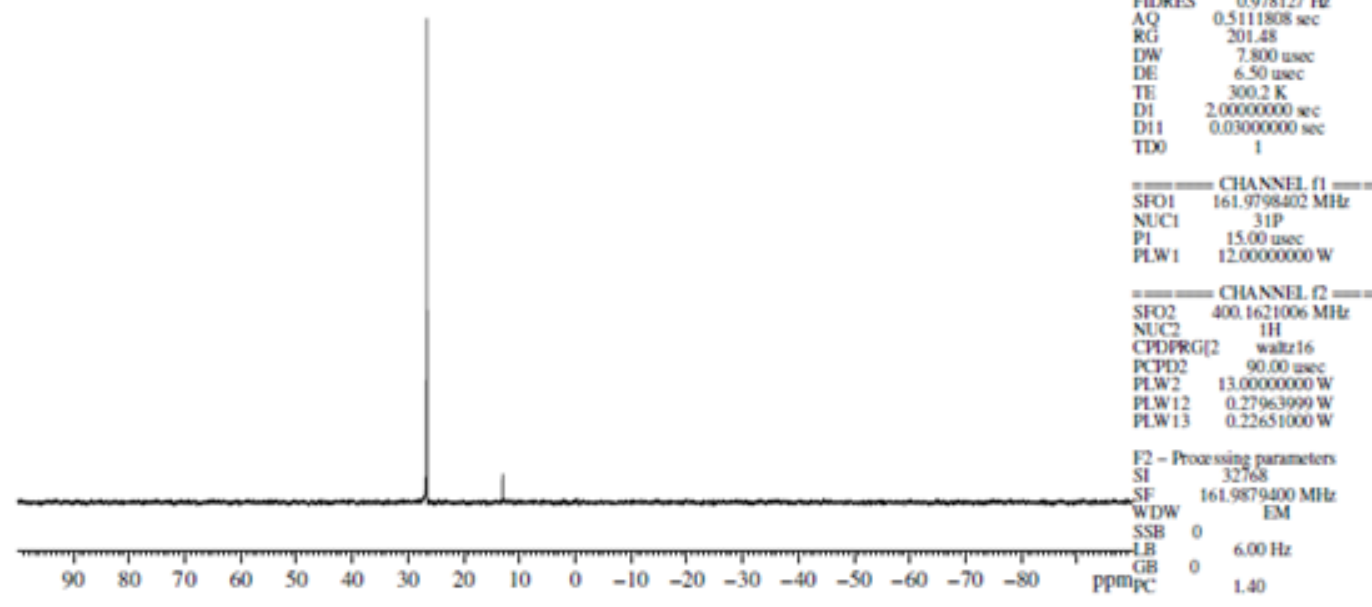

Figure 109: ${ }^{31} \mathrm{P}$ NMR spectrum of $3 p$

NRAT-IV-189B

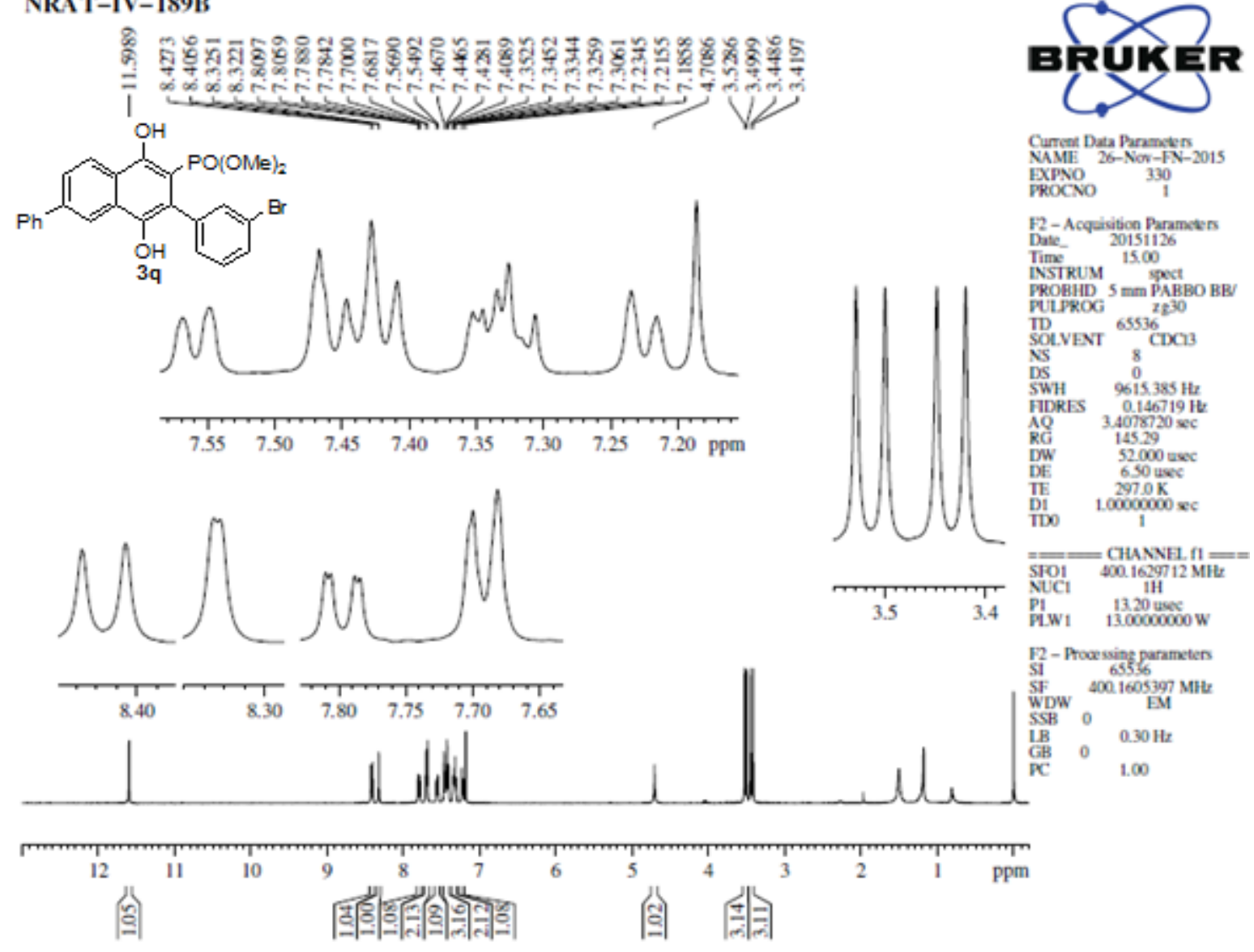

Figure 110: ${ }^{1} \mathrm{H}$ NMR spectrum of $3 q$ 


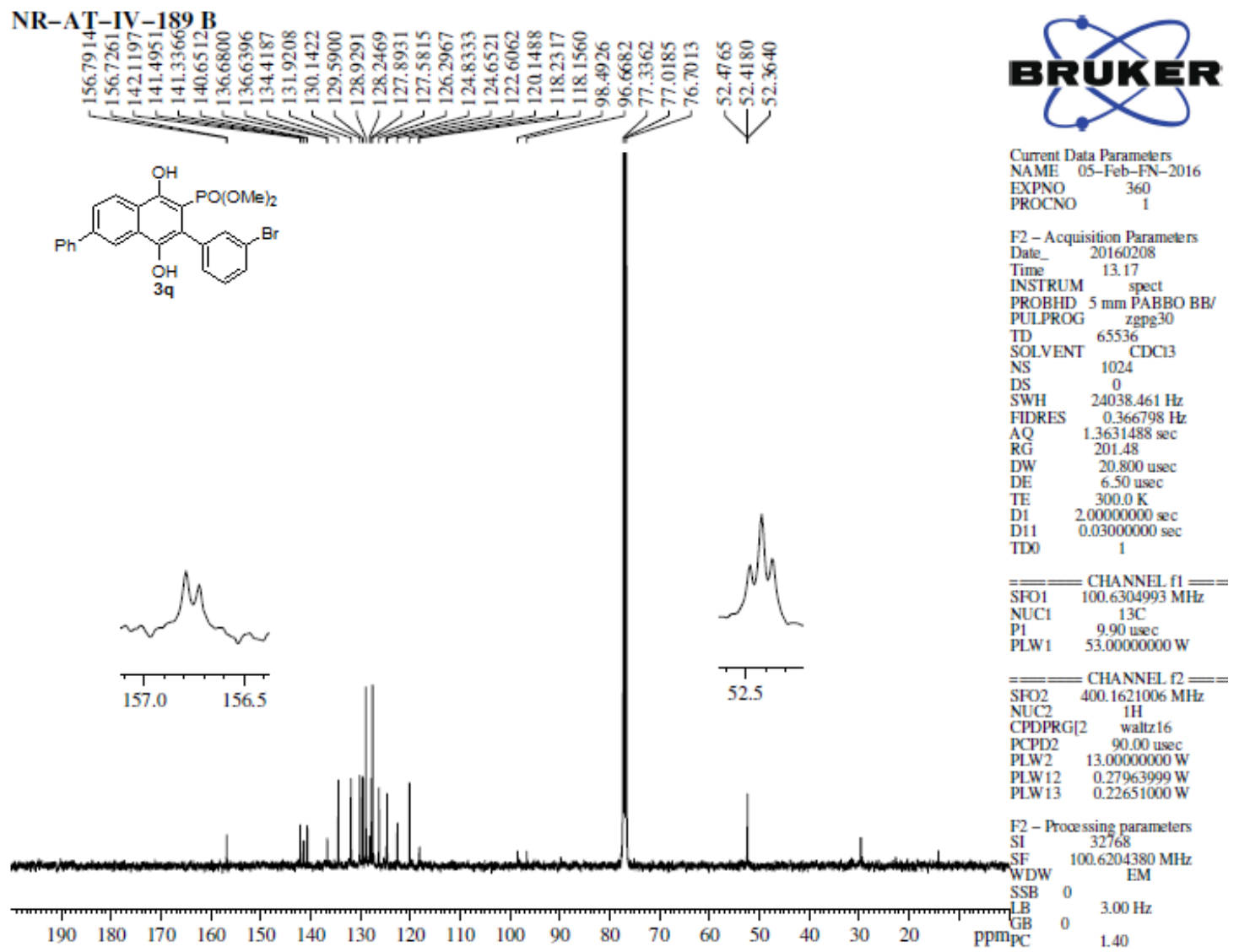

Figure 111: ${ }^{13} \mathrm{C}$ NMR spectrum of $3 q$
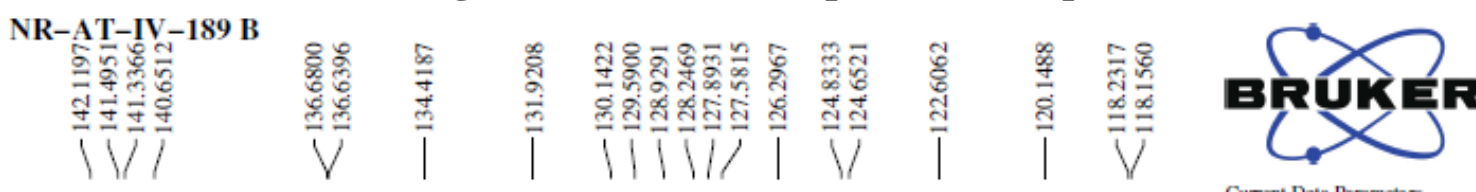

Current Data Parameters $\begin{array}{lr}\text { EXPNO } & 360 \\ \text { PROCNO } & 1\end{array}$

F2 - Acquisition Parameters

$\begin{array}{ll}\text { Date_ } & 20160208 \\ \text { Time } & 13.17\end{array}$

Time
INSTRUM 13.17
spect

PROBHD 5 mm PABBO B

$\begin{array}{ll}\text { PD } & \text { Tgpg 30 } \\ \text { SOLVENT } & \text { CD536 } \\ \text { CDC13 }\end{array}$

$\begin{array}{lc}\text { NS } & 1024 \\ \text { DS } & 0 \\ \text { SWH } & 24038.461 \mathrm{~Hz}\end{array}$

FO 13631488 sec

RG $\quad 201.48$

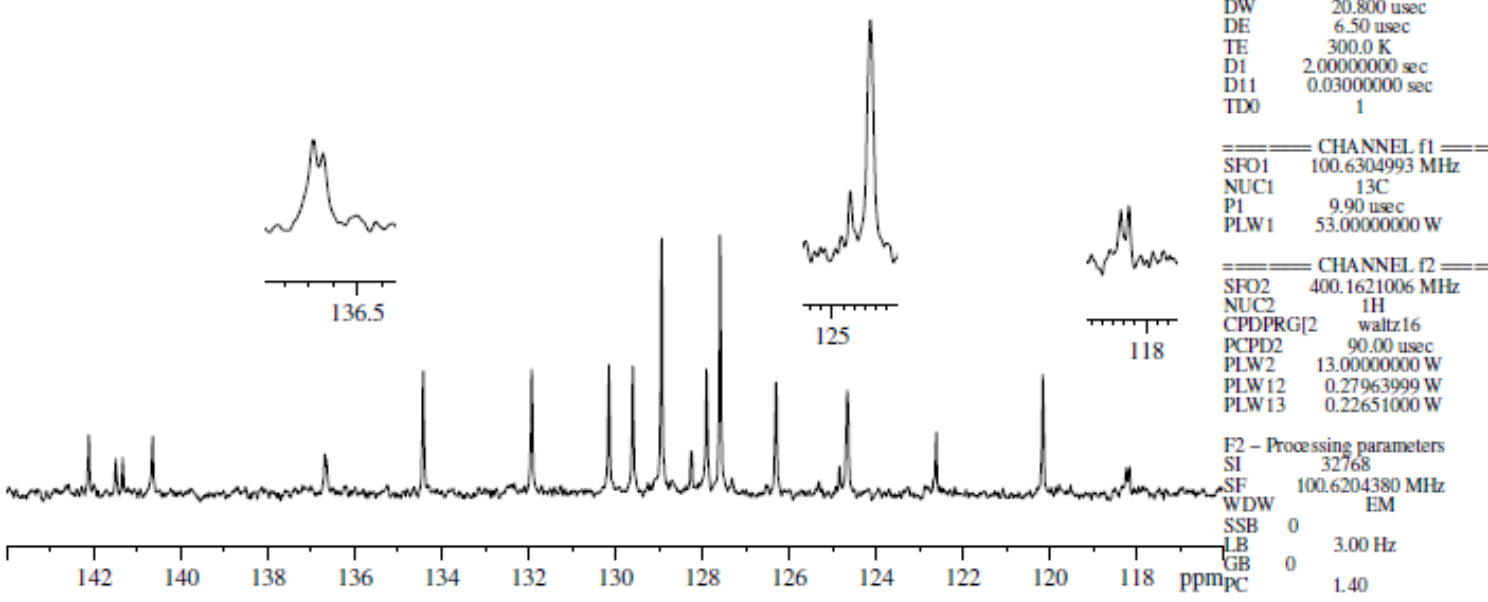

Figure 112: ${ }^{13} \mathrm{C}$ NMR spectrum of 3q (expansion) 


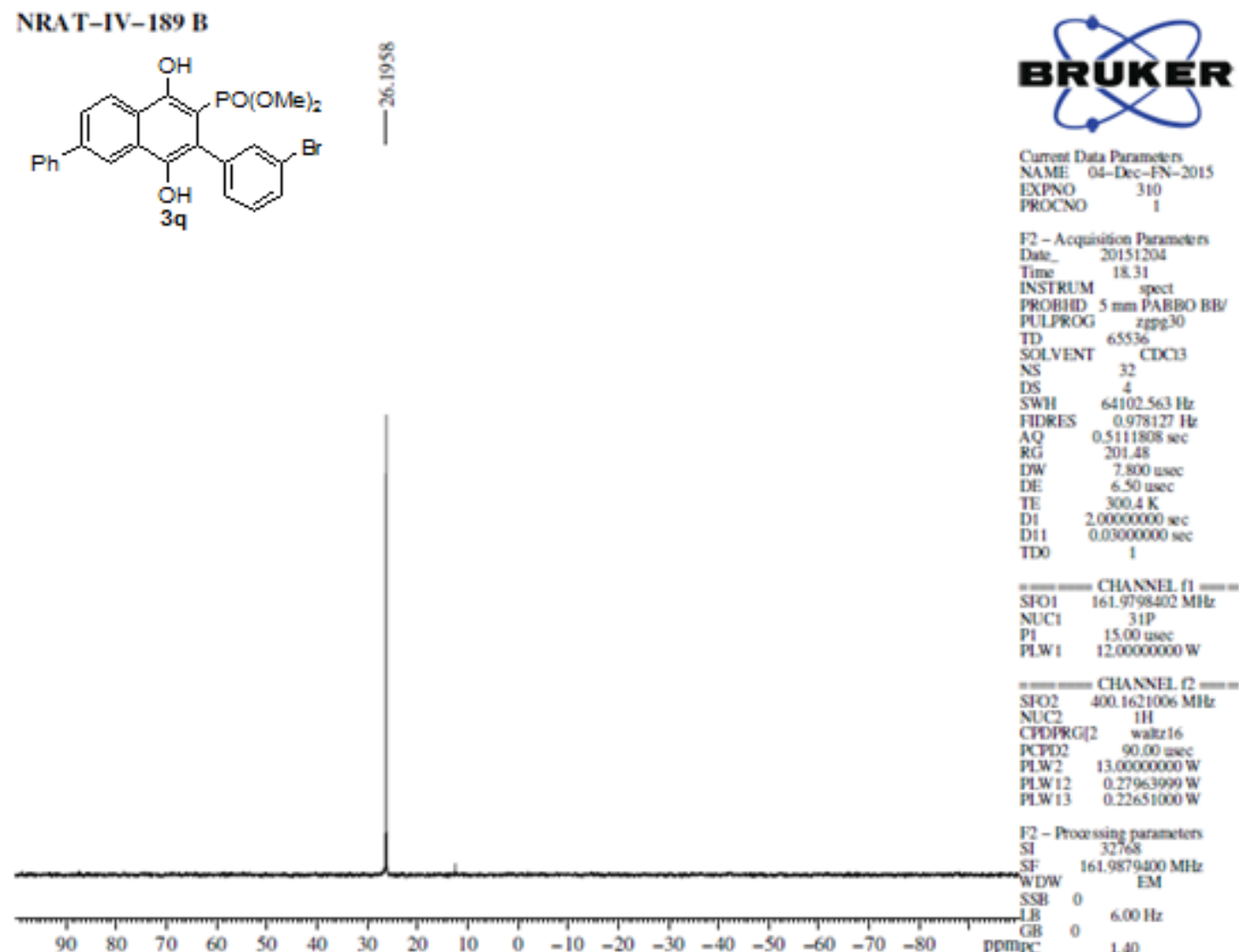

Figure 113: ${ }^{31} \mathrm{P}$ NMR spectrum of $3 q$

NRAT-IV-177

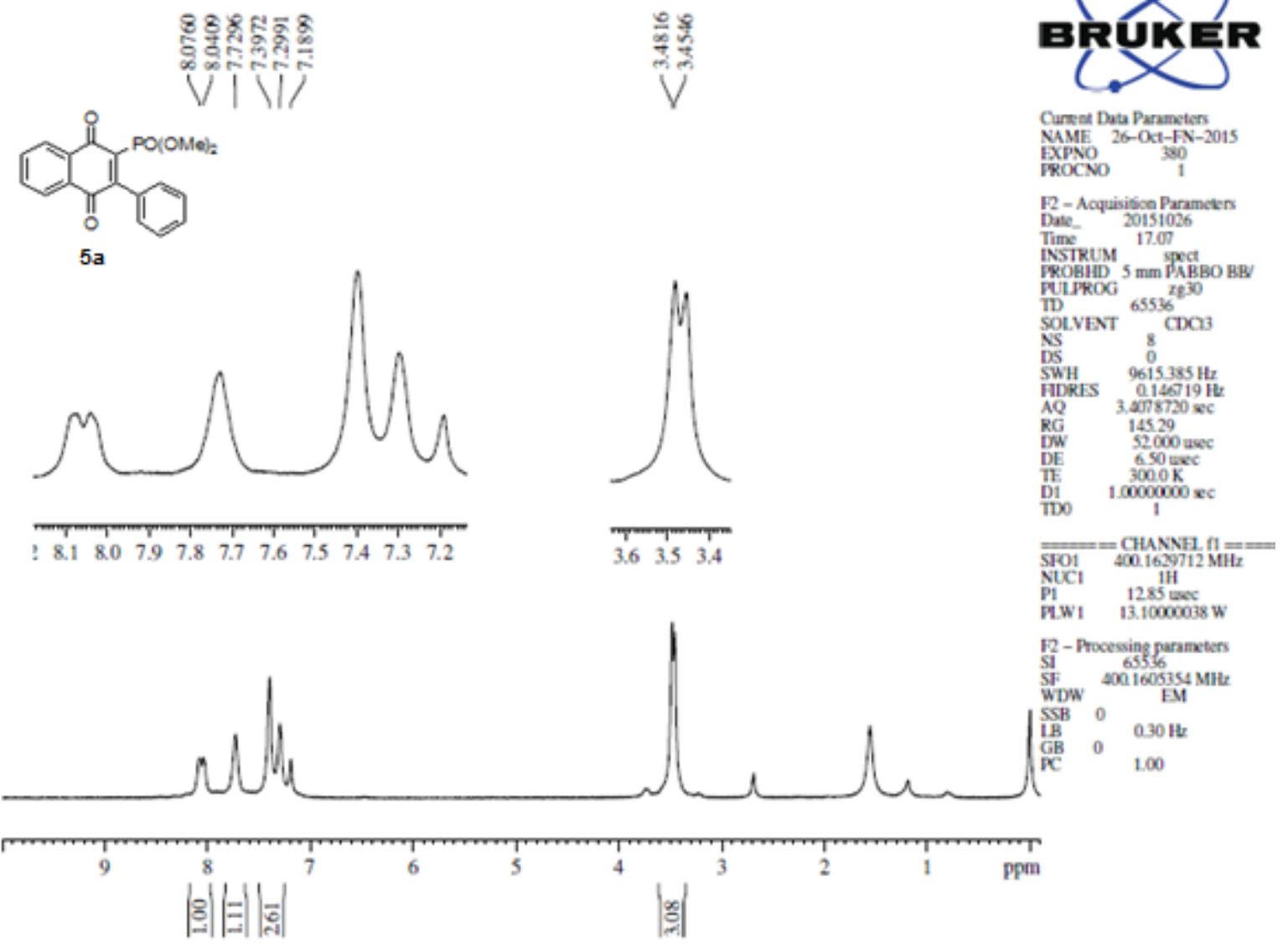

Figure 114: ${ }^{1} \mathrm{H}$ NMR spectrum of $5 a$ 


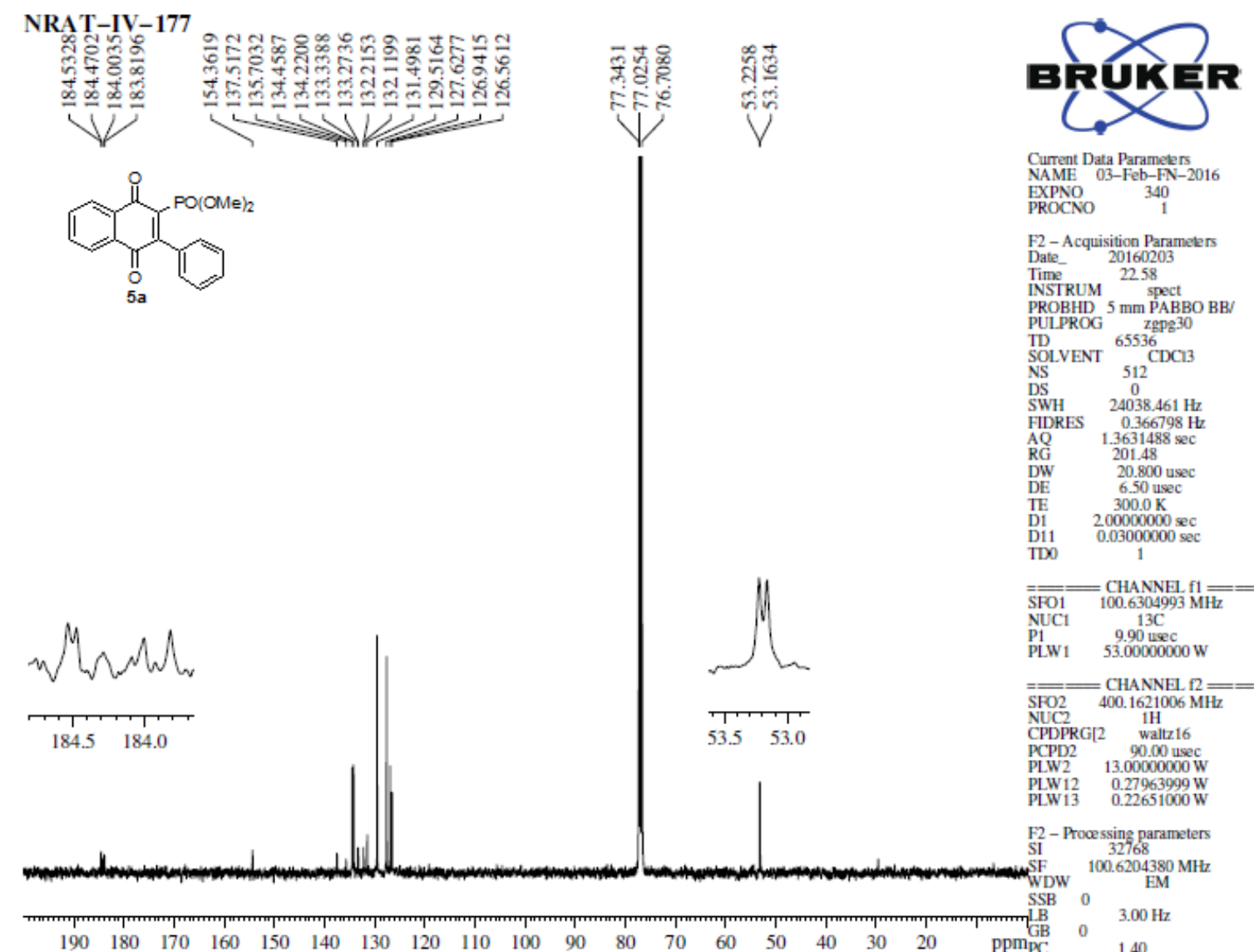

Figure 115: ${ }^{13} \mathrm{C}$ NMR spectrum of $5 \mathrm{a}$
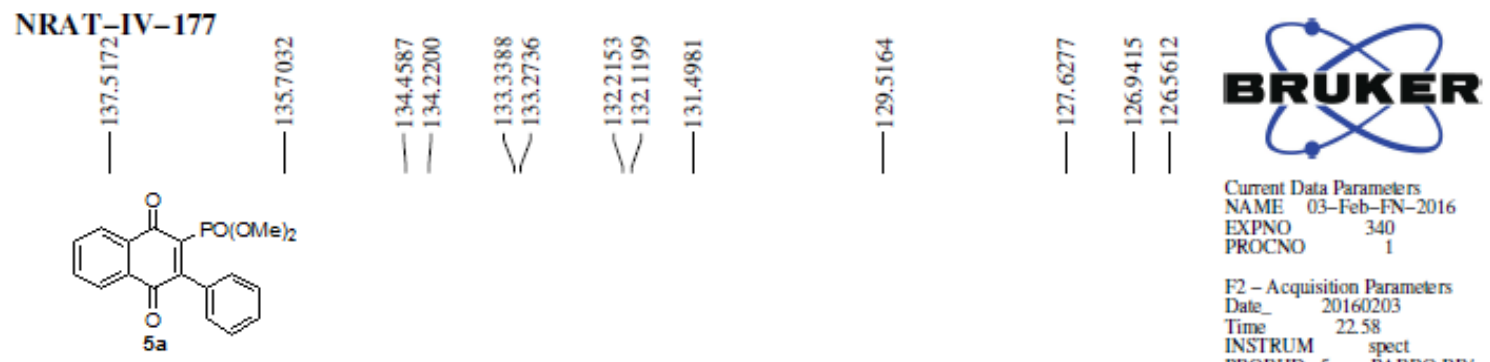

Current Data Parameters
NAME $03-F e b-F N-2016$ $\begin{array}{lc}\text { NAME } & \text { 03-Feb-FN } \\ \text { EXPNO } & 340\end{array}$

F2 - Acquisition Parameters

Date_ 20160203

Time 22.58

INSTRUM spect
PROBHD $5 \mathrm{~mm}$ PABBO BB

$\underset{\text { PULPROG }}{\text { PROBHD }} 5 \mathrm{~mm}$ PABBO

$\begin{array}{lr}\text { PULPROG } & \text { zgpg 30 } \\ \text { TD } & 65536 \\ \text { SOLVENT } & \text { CDC13 }\end{array}$

$\begin{array}{lcl}\text { NS } & 512 \\ \text { DS } & 0 \\ \text { SWH } & 24038.461 \mathrm{H}\end{array}$

$\begin{array}{lr}\text { SWH } & 24038.461 \mathrm{~Hz} \\ \text { FIDRES } & 0.366798 \mathrm{~Hz}\end{array}$

$\begin{array}{ll}\text { FIDRES } & 0.366798 \mathrm{H} \\ \text { AQ } & 1.3631488 \mathrm{sec}\end{array}$

$\begin{array}{ll}\text { AQ } & 1.36314 \\ \text { RG } & 201.48\end{array}$

$\begin{array}{ll}\text { DW } & 20.800 \text { usec } \\ \text { DE } & 6.50 \text { usec }\end{array}$

TE $\quad 300.0 \mathrm{~K}$

D1 200000000

D11 $0.03000000 \mathrm{sec}$

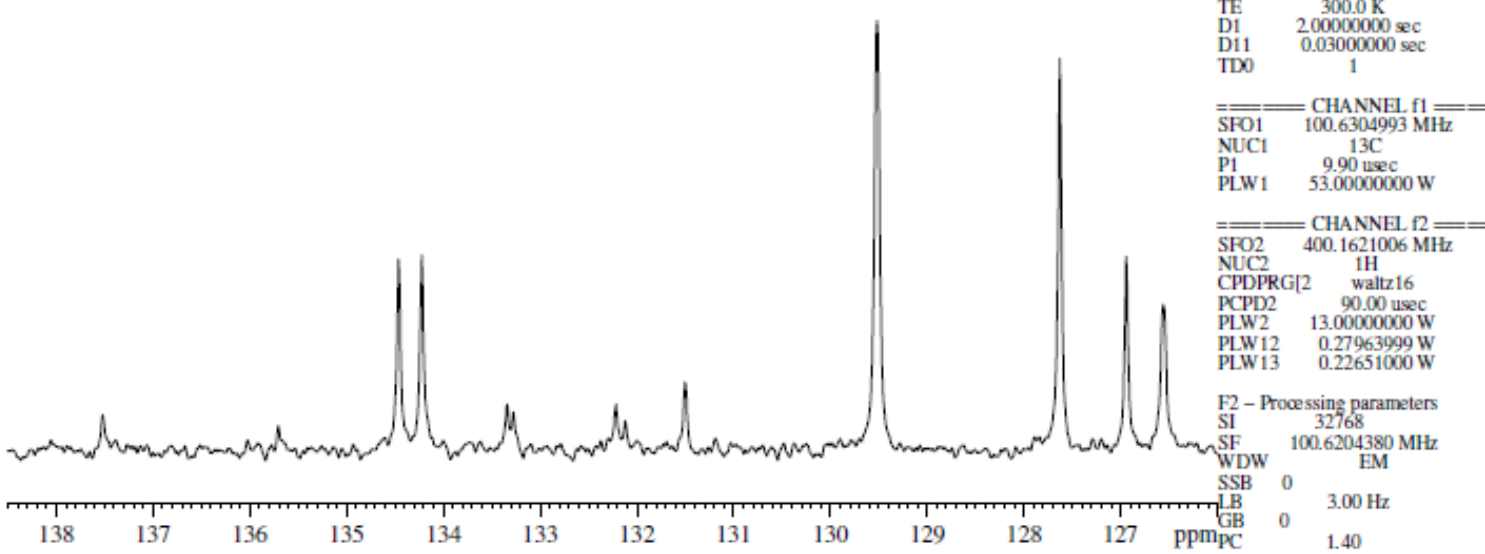

Figure 116: ${ }^{13} \mathrm{C}$ NMR spectrum of 5 a (expansion) 
NRAT IV 177<smiles>COC1=C(c2ccccc2)C(=O)C(=O)c2ccccc21</smiles>

Current Data Paramelers EXPNO
PROCNO

F2 - Acquisition Parameters

Date_ 2016011

Time

PROBHD $5 \mathrm{~mm}$ PABBO BB

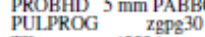

TD 65536

${ }_{\text {SOLVNT }}{ }_{32} \mathrm{CDC}$

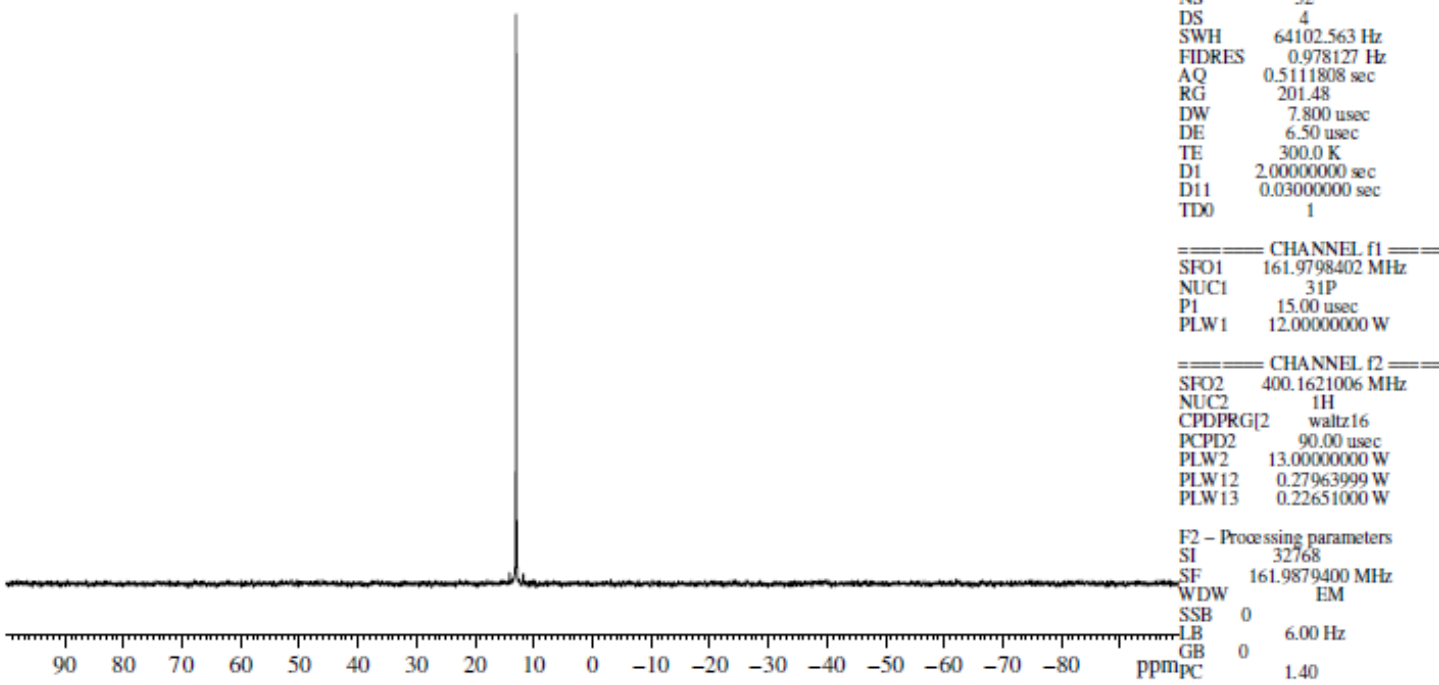

Figure 117: ${ }^{31} \mathrm{P}$ NMR spectrum of 5a

NRAT-IV-157

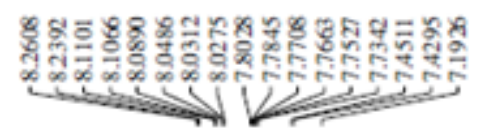<smiles>COC(=O)C1=C(c2ccc([N+](=O)[O-])cc2)C(=O)c2ccccc2C1=O</smiles>

$5 b$

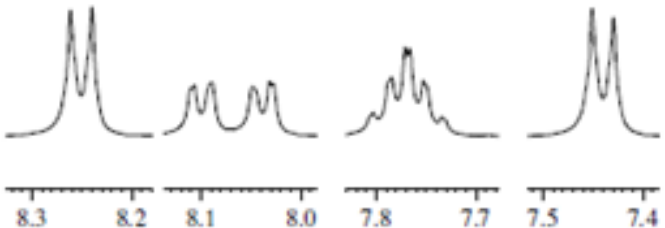

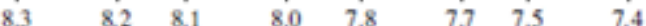

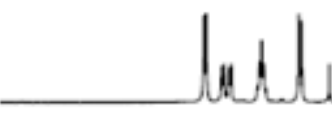
细首目园

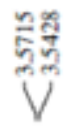

BRUKER

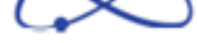

Cursent Dat Parametes XXis $07-0 \mathrm{x}-1 \mathrm{~N}-201$ DXPNO NO

12-Acquinition Parrenctes

$\begin{array}{ll}\text { Date- } & 20151007 \\ \text { Time } & 15.17\end{array}$

NSTRLM sect

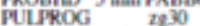

TD 65536

SOLVENT CDCl

Ds

SWH $9615385 \mathrm{~Hz}$

HDRES 014619i

RG $\quad 3.5078720$

RG
DW
DE
DE

Dine $\quad 1.0000000$

Fo1 - CWUNNE $\mathrm{f1}=$

Nuc1 $1 \mathrm{H}$

$1320 \mathrm{usec}$
Ww1 $13000000 \mathrm{w}$

P2 - Processing punneters

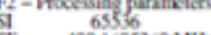

ST $4001605360 \mathrm{Mllz}$

SSB 0 EM

IB $0.30 \mathrm{H}$

$\begin{array}{lll}\text { GB } & 0 & 030 \\ \text { RC } & 100\end{array}$

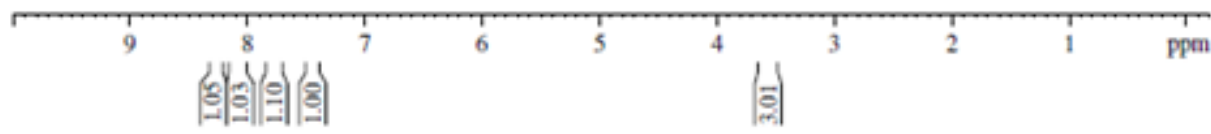

Figure 118: ${ }^{1} \mathrm{H}$ NMR spectrum of $5 \mathrm{~b}$ 


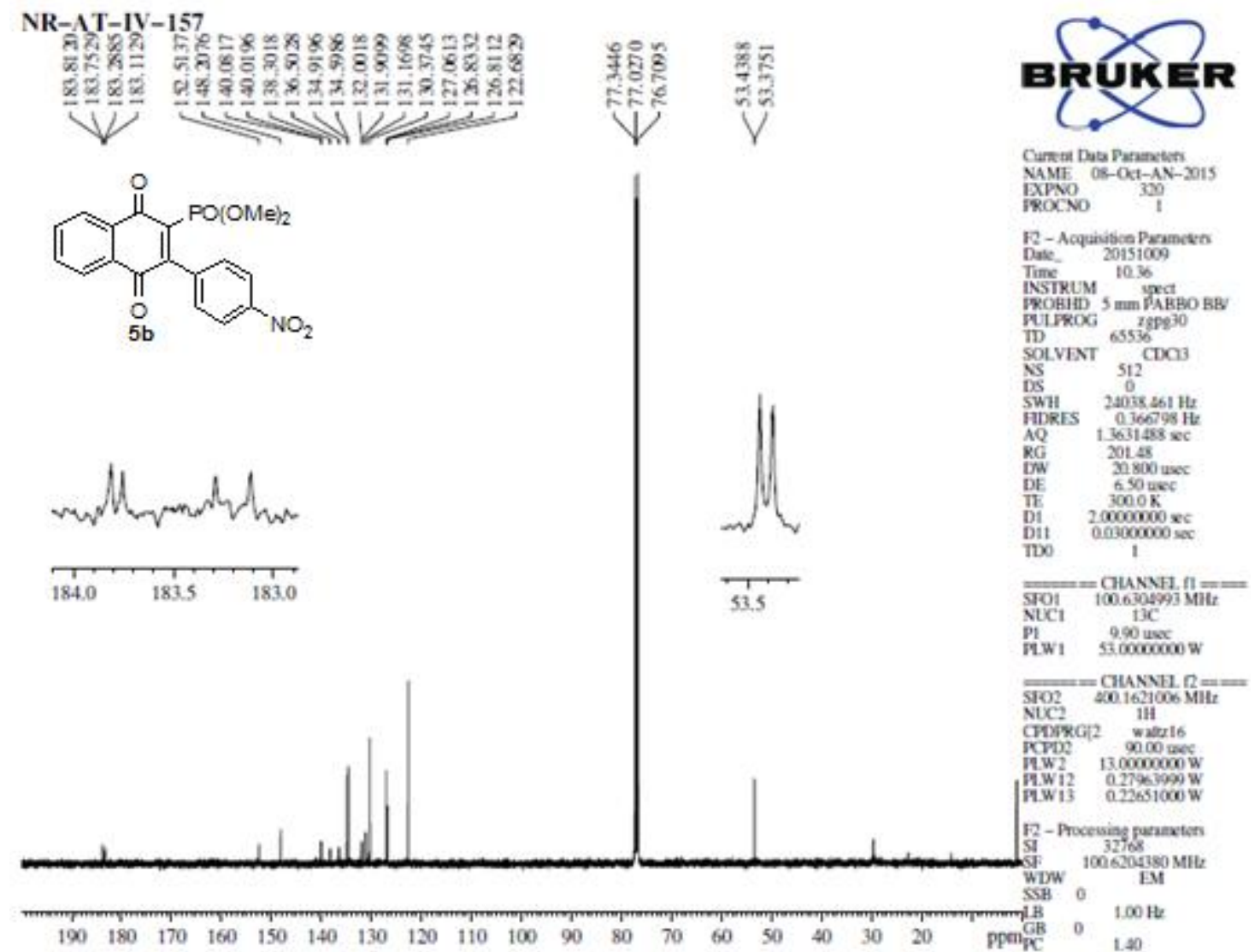

Figure 119: ${ }^{13} \mathrm{C}$ NMR spectrum of $5 b$

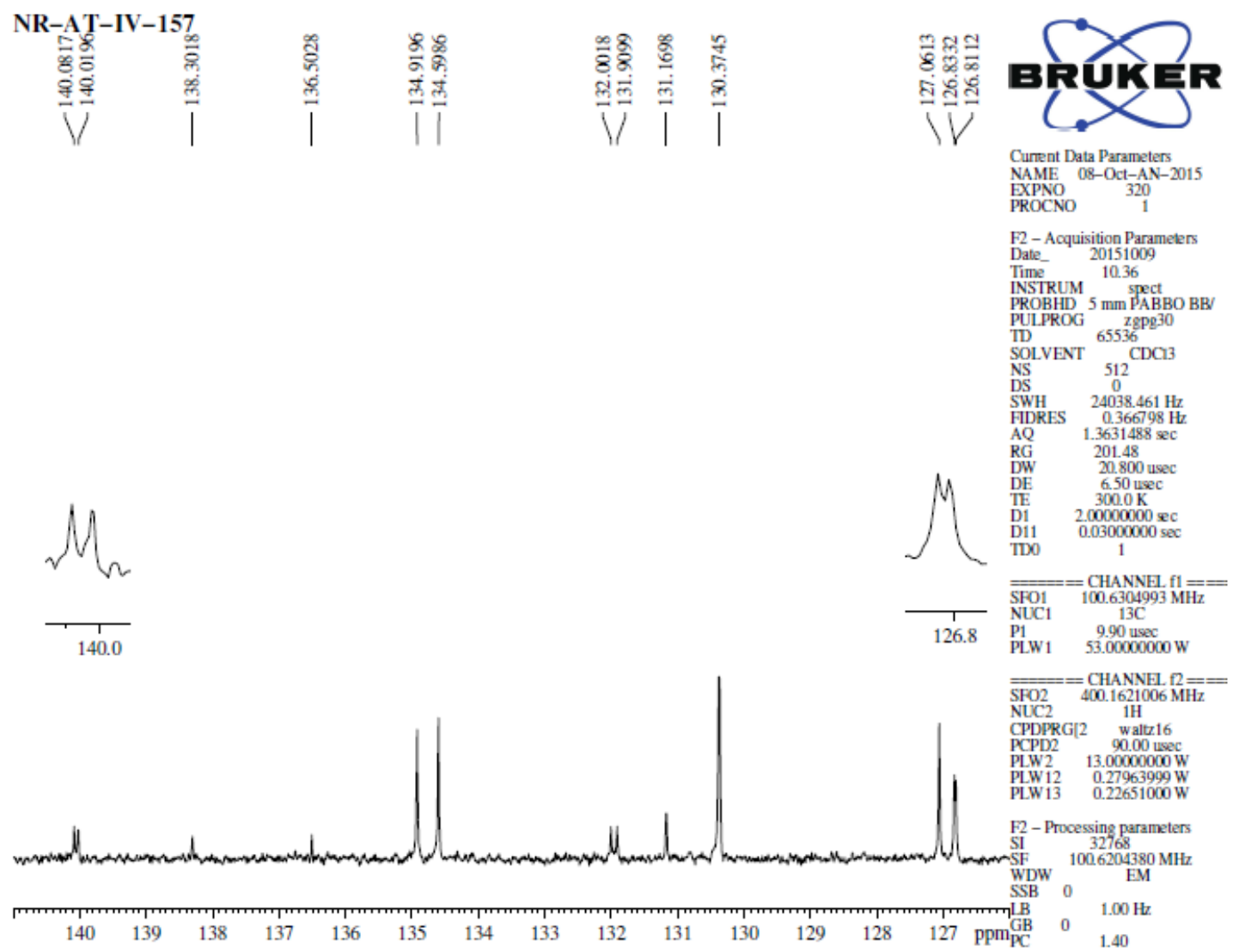

Figure 120: ${ }^{13} \mathrm{C}$ NMR spectrum of $5 b$ (expansion) 
<smiles>COC1=C(c2ccc([N+](=O)[O-])cc2)C(=O)c2ccccc2C1=O</smiles>

Cursent Data Punameters
NME 28-Sep-FN-2015

EXPNO 390

एROC

F2-Acquisition Parameten

Date- 2015092

INSIRLM s Pect

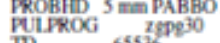

TD 65536

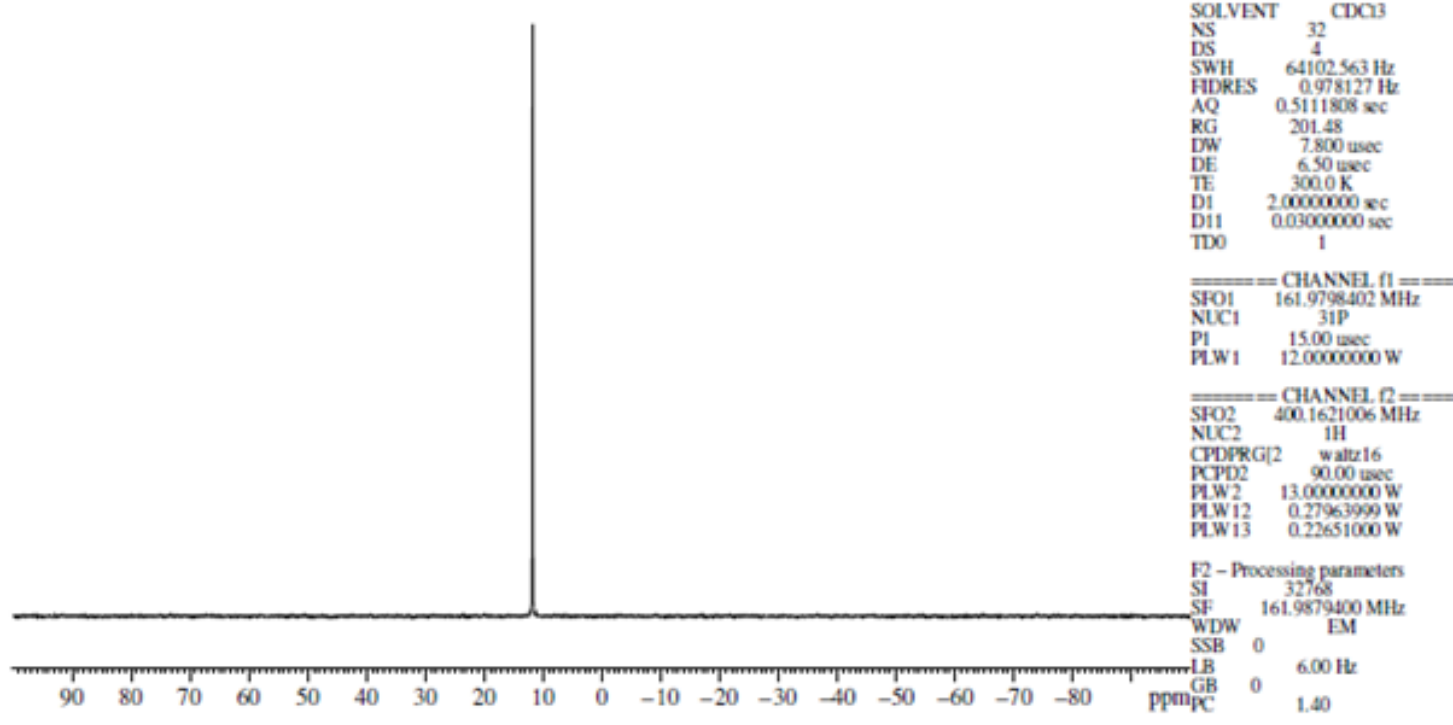

Figure 121: ${ }^{31} \mathrm{P}$ NMR spectrum of $5 \mathrm{~b}$

NRAT-IV-164<smiles>COC1=C(c2ccc(F)cc2)C(=O)c2ccccc2C1=O</smiles>
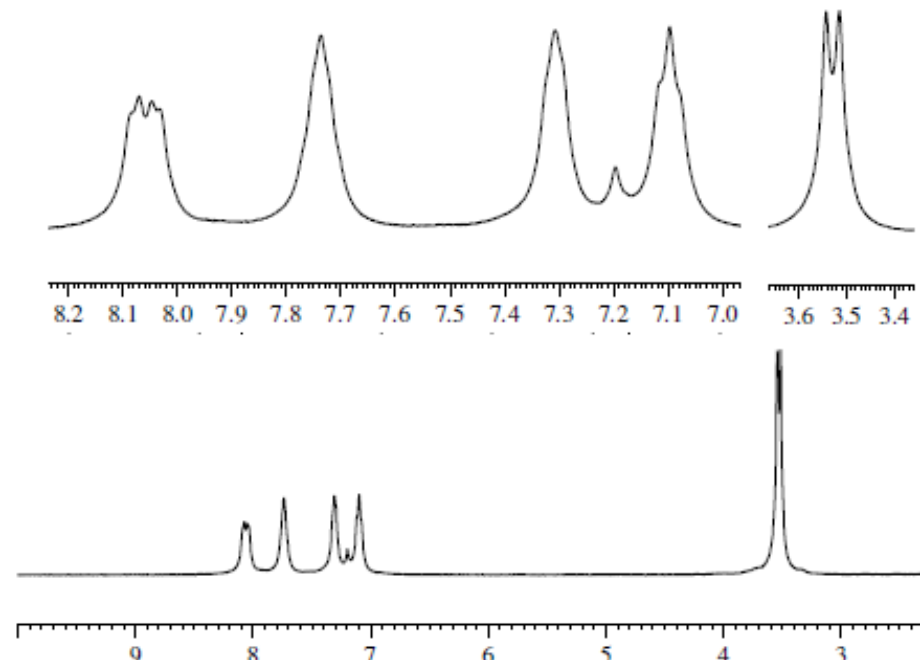
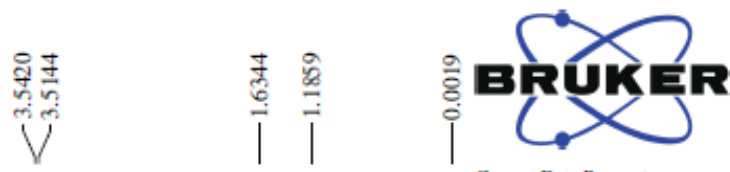

Current Data Parameters
NAME $06-$ Oct-FN-2015 EXPNO $\quad 370$

F2 - Acquisition Parameters

$\begin{array}{lc}\text { Date_ } & 20151006 \\ \text { Time } & 15.18\end{array}$

INSTRUM 15.18 spect PROBHD $5 \mathrm{~mm}$ PABBO BE TD 65536 SOLVENT $\quad$ CDC13 $\begin{array}{ll}\text { NS } & 8 \\ \text { DS } & 0\end{array}$ DS $\quad 00.285 \mathrm{~Hz}$ FIDRES $\quad 0.146719 \mathrm{H}$ AQ $\quad 3.4078720 \mathrm{sec}$

$\begin{array}{ll}\text { RG } & 114.26 \\ \text { DW } & 52.000 \text { usec }\end{array}$

DE 6.50 usec $\begin{array}{lr}\text { TE } & 300.0 \mathrm{~K} \\ \mathrm{D} 1 & 1.00000000 \mathrm{se}\end{array}$ $=\underset{\text { SFO1 }}{=}=\underset{400.1629712 \mathrm{MHz}}{\text { CHANEL }}=$ $\begin{array}{ll}\text { P1 } & 13.20 \text { usec } \\ \text { PLW1 } & 13.00000000 \mathrm{~W}\end{array}$ $\begin{array}{ll}\text { F2 - Prowssing parameters } \\ \text { S1 } & 65536 \\ \text { SF } & 400.1605339 \mathrm{MHz}\end{array}$ $\begin{array}{lc}\mathrm{SF} & 400.1605339 \mathrm{Mr} \\ \mathrm{WDW} & \mathrm{EM}\end{array}$ $\begin{array}{lll}\text { SSB } & 0 & 0.30 \mathrm{~Hz} \\ \text { IB }\end{array}$ $\mathrm{PC}$ 1.00

Figure 122: ${ }^{1} \mathrm{H}$ NMR spectrum of $5 \mathrm{c}$ 


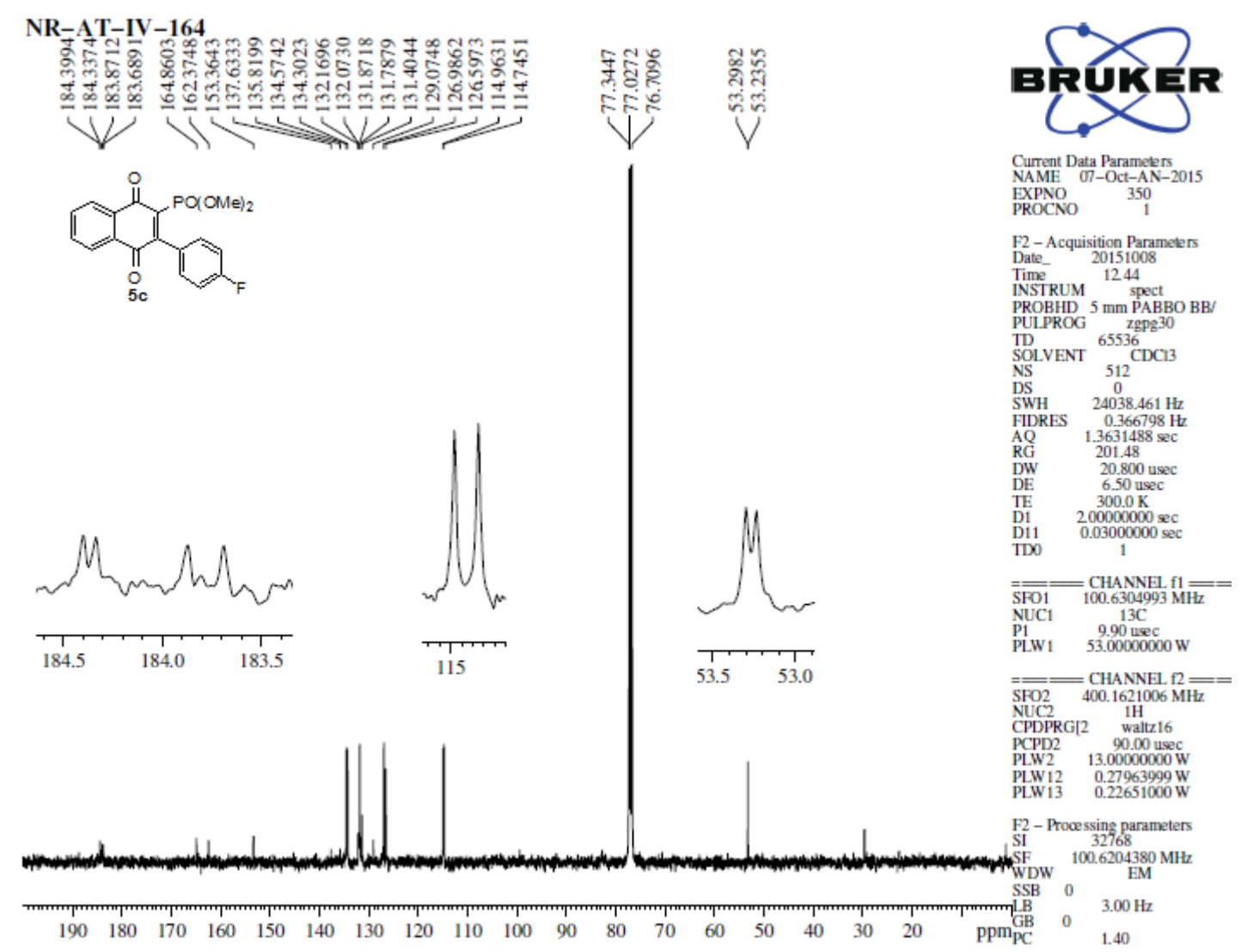

Figure 123: ${ }^{13} \mathrm{C}$ NMR spectrum of $5 \mathrm{c}$

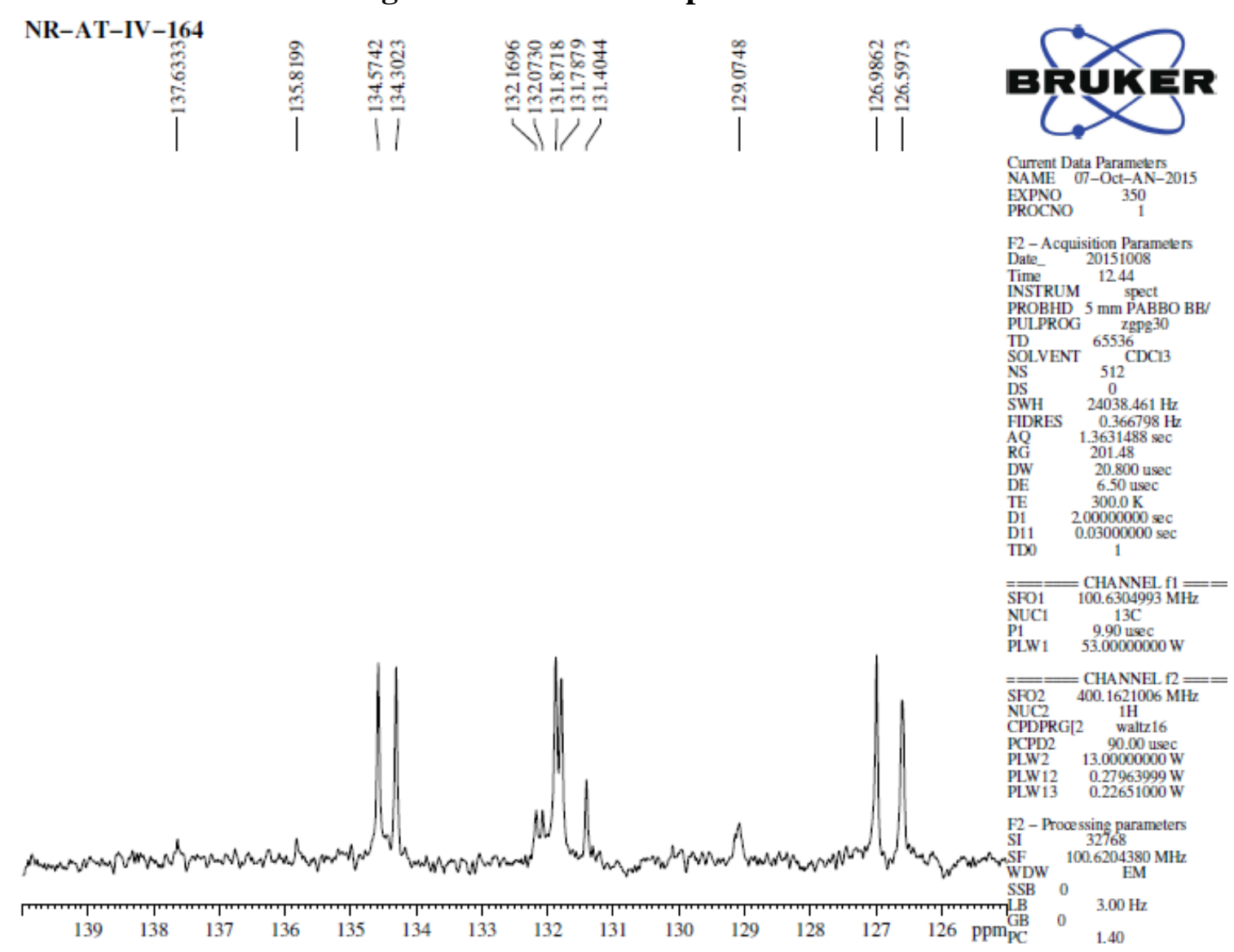

Figure 124: ${ }^{13} \mathrm{C}$ NMR spectrum of $5 \mathrm{c}$ (expansion) 


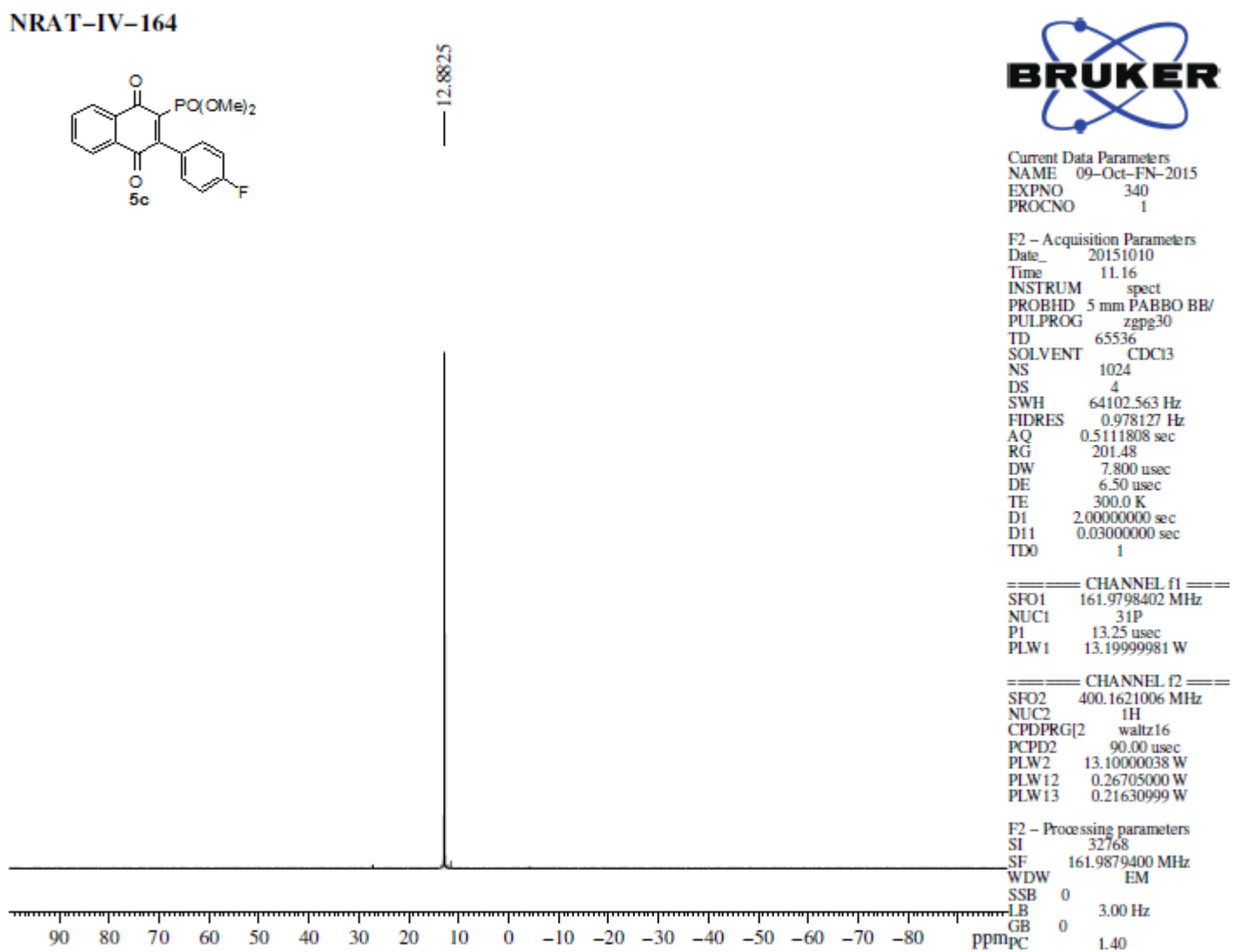

Figure 125: ${ }^{31} \mathrm{P}$ NMR spectrum of $5 \mathrm{c}$

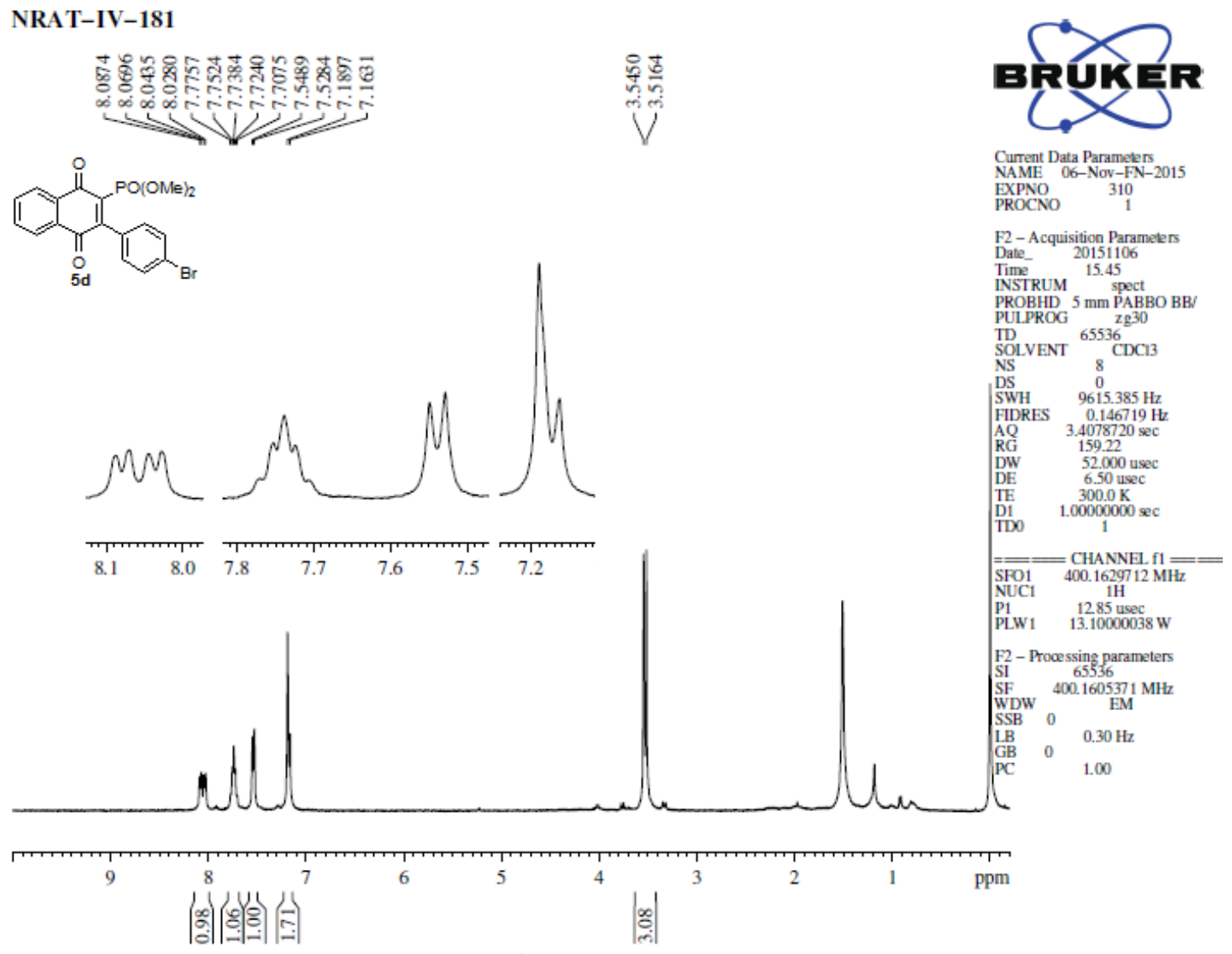

Figure 126: ${ }^{1} \mathrm{H}$ NMR spectrum of $5 \mathrm{~d}$ 


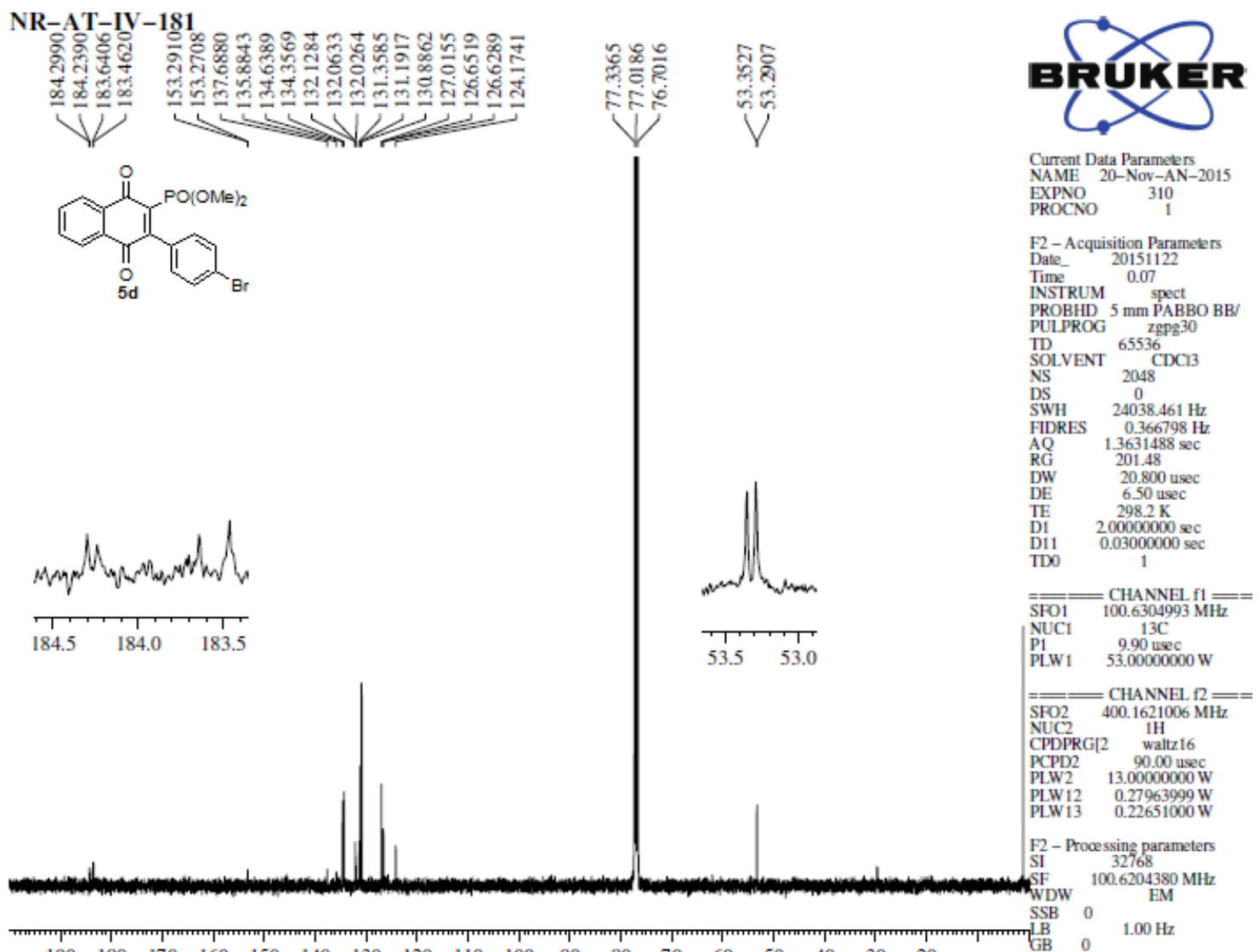

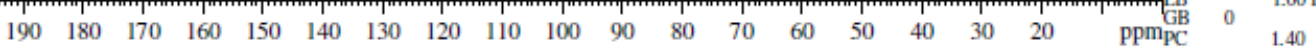

Figure 127: ${ }^{13} \mathrm{C}$ NMR spectrum of $5 d$
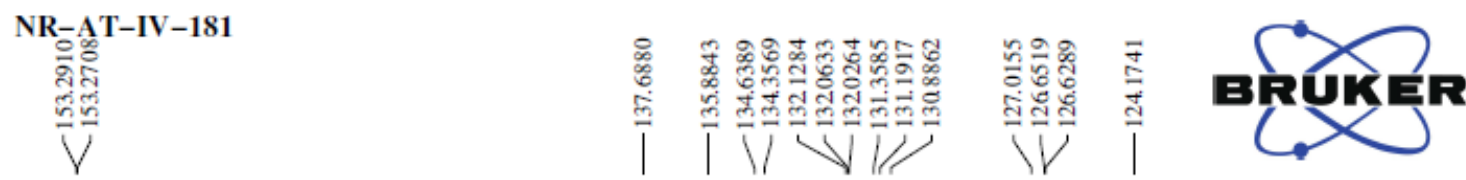

Current Data Parameters 2015

EXPNO

F2 - Acquisition Parameters

F2-Acquisition Param
Date__ 20151122

Time 0.07

INSTRUM spect

PROBHD $5 \mathrm{~mm}$ PABBO B

PULPROG Z $7 \mathrm{gPg} 30$

${ }_{\text {SDLVENT }}^{65536} \mathrm{CDC13}$

NS 2048

$\begin{array}{ll}\text { DS } & 0 \\ \text { SWH } & 24038.461 \mathrm{~Hz}\end{array}$

FIDRES $\quad 0.366798 \mathrm{~Hz}$

AQ $\quad 1.3631488 \mathrm{sec}$

DW $\quad 20.800$ usec

mang M

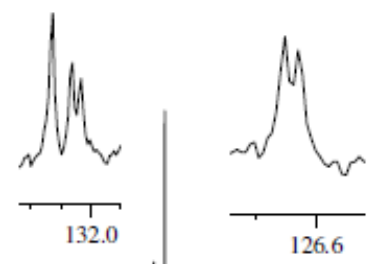

$98.2 \mathrm{~K}$

$\begin{array}{ll}\text { D1 } & 200000000 \mathrm{sec} \\ \text { Di1 } & 0.03000000 \mathrm{sec}\end{array}$

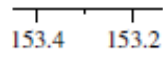

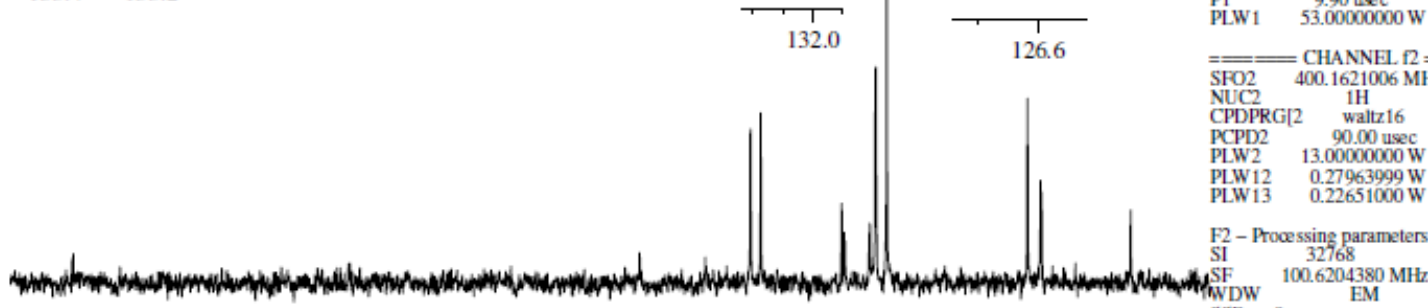
$\overline{\bar{S}} \overline{\overline{F O 1}}={ }_{100.6304993 \mathrm{M}}^{\text {CH }} \mathrm{F}=$ NUC1 $13 \mathrm{C}$

$\begin{array}{ll}\text { P1 } & 9.90 \text { usec } \\ \text { PLW1 } & 53.00000000 \mathrm{~W}\end{array}$

$=$ CHANNEL $\mathrm{f}_{2}=$

SFO2 $400.1621006 \mathrm{MHz}$

CPDPRG 2 waltz 16

$\begin{array}{ll}\mathrm{PCPDD} 2 & 90.00 \text { usec } \\ \mathrm{PLW} 2 & 13.00000000 \mathrm{~W}\end{array}$

PLW12

oow

F2 - Prooessing parameters

Figure 128: ${ }^{13} \mathrm{C}$ NMR spectrum of $5 \mathrm{~d}$ (expansion) 


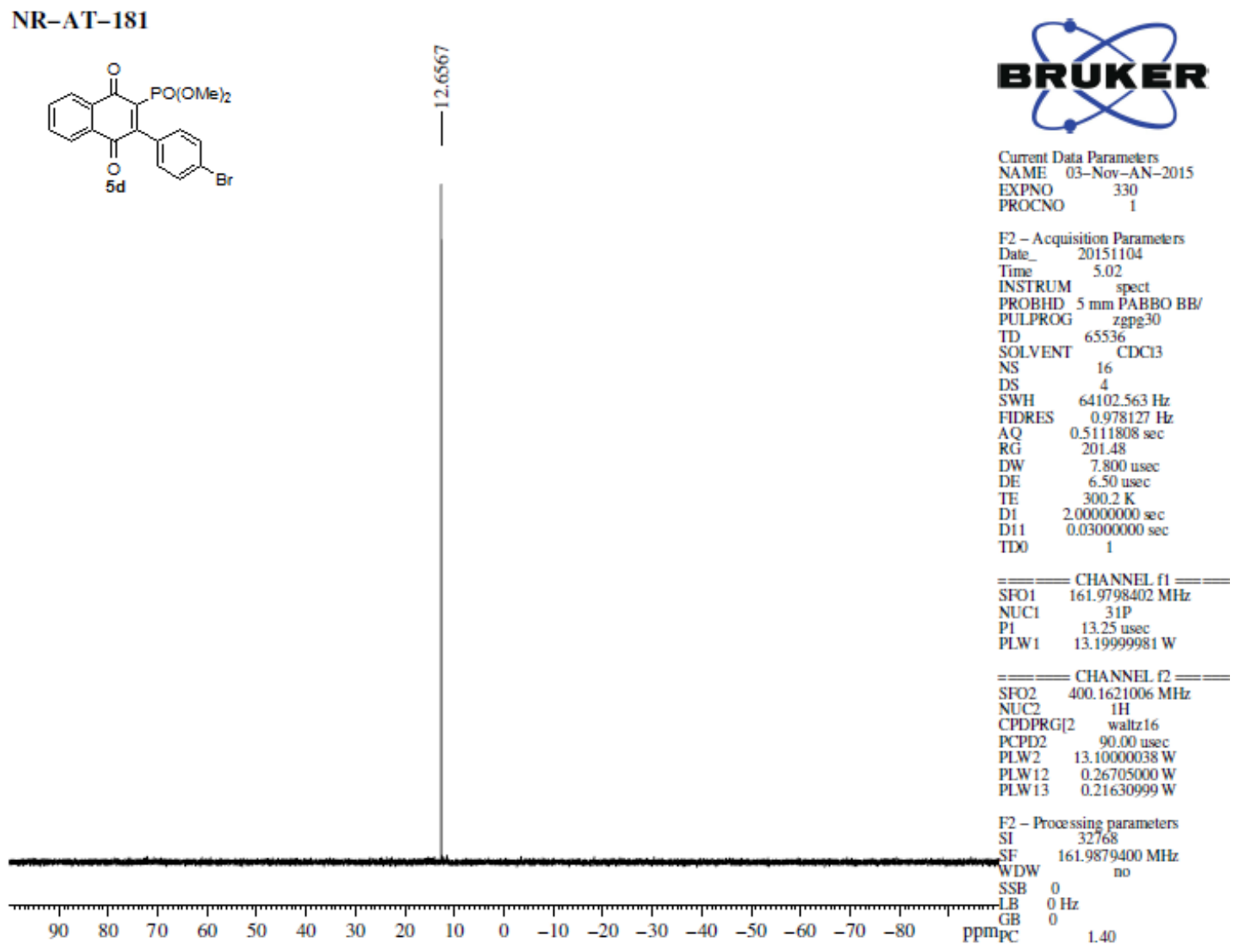

Figure 129: ${ }^{31} \mathrm{P}$ NMR spectrum of $5 \mathrm{~d}$

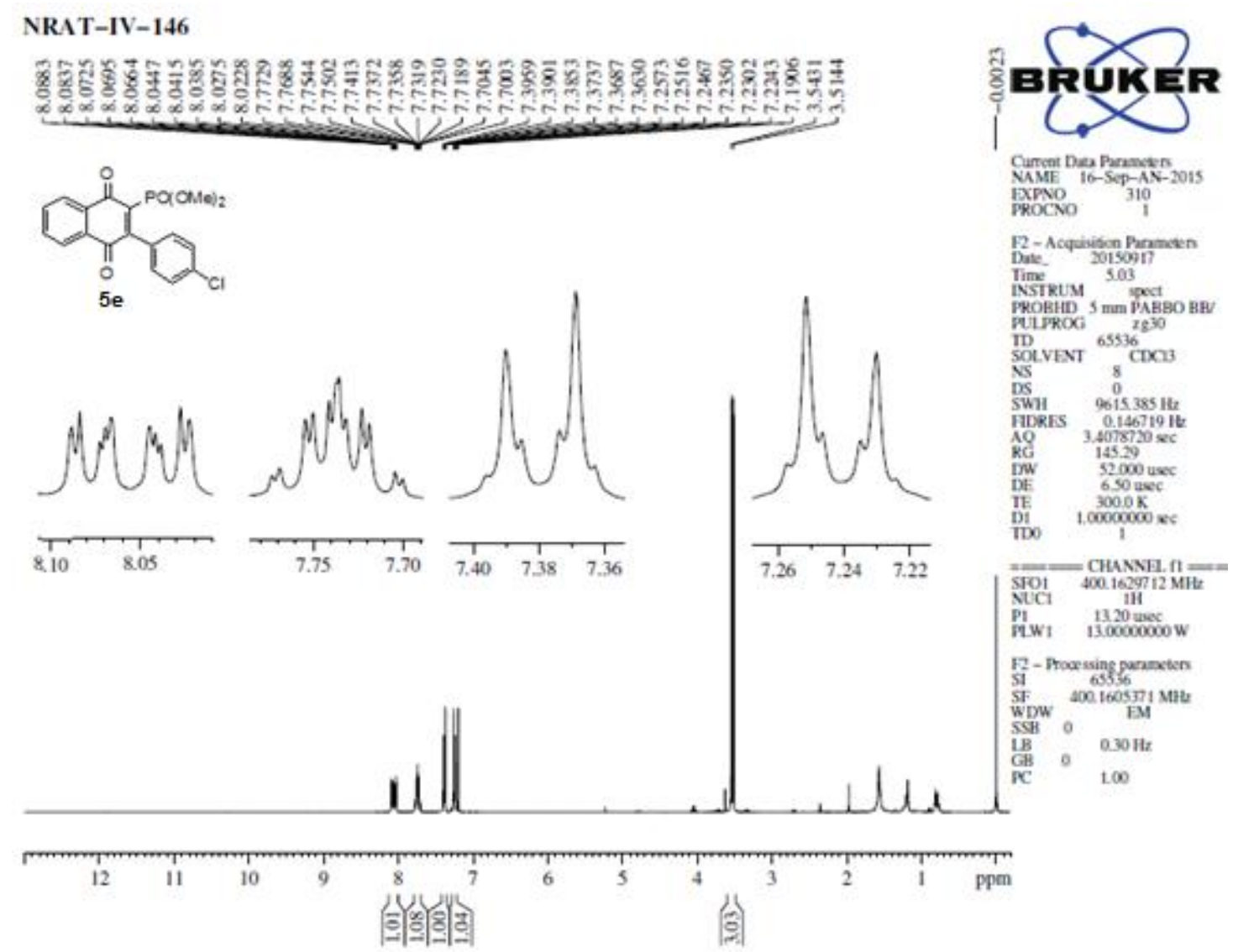

Figure 130: ${ }^{1} \mathrm{H}$ NMR spectrum of $5 \mathrm{e}$ 


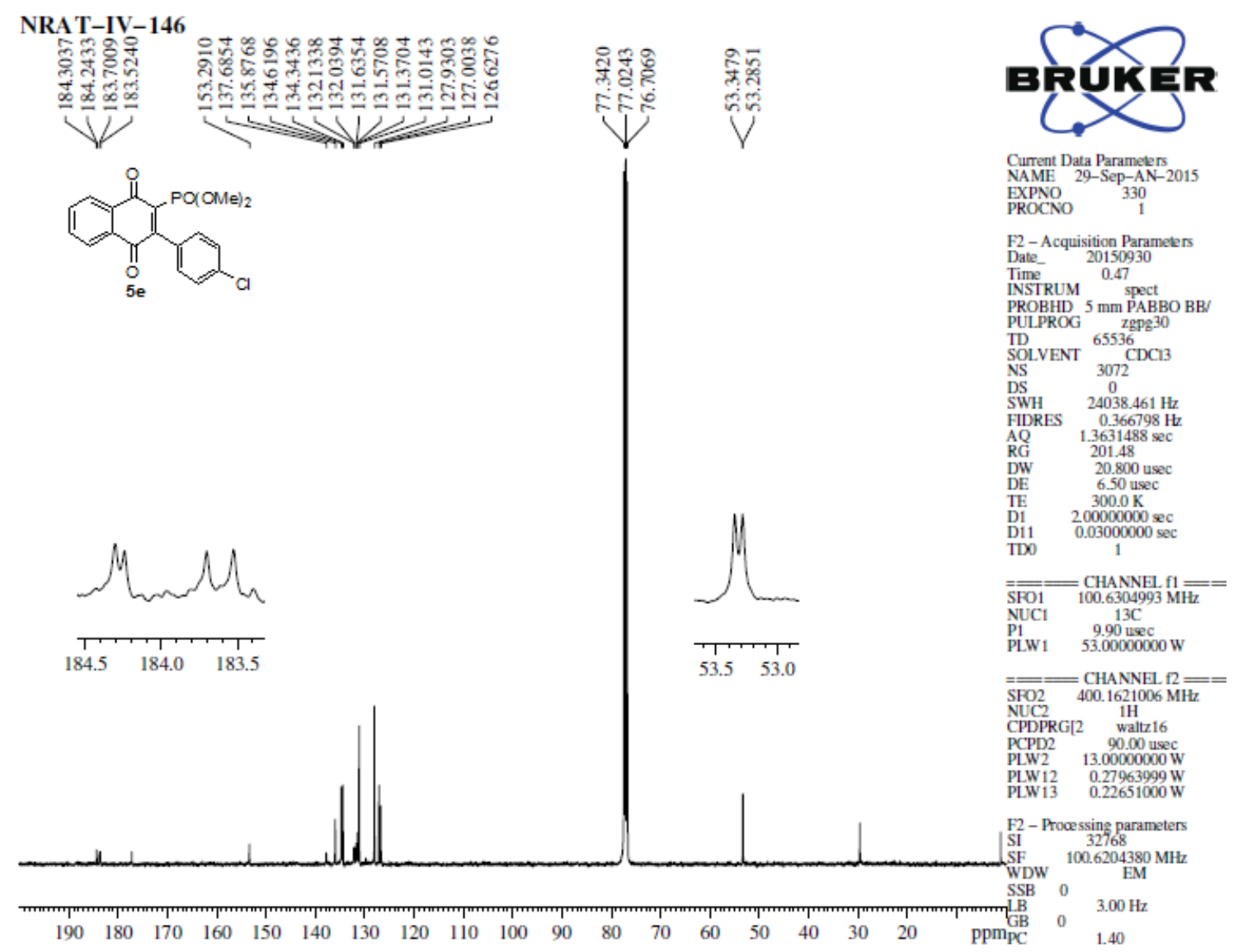

Figure 131: ${ }^{13} \mathrm{C}$ NMR spectrum of $5 \mathrm{e}$

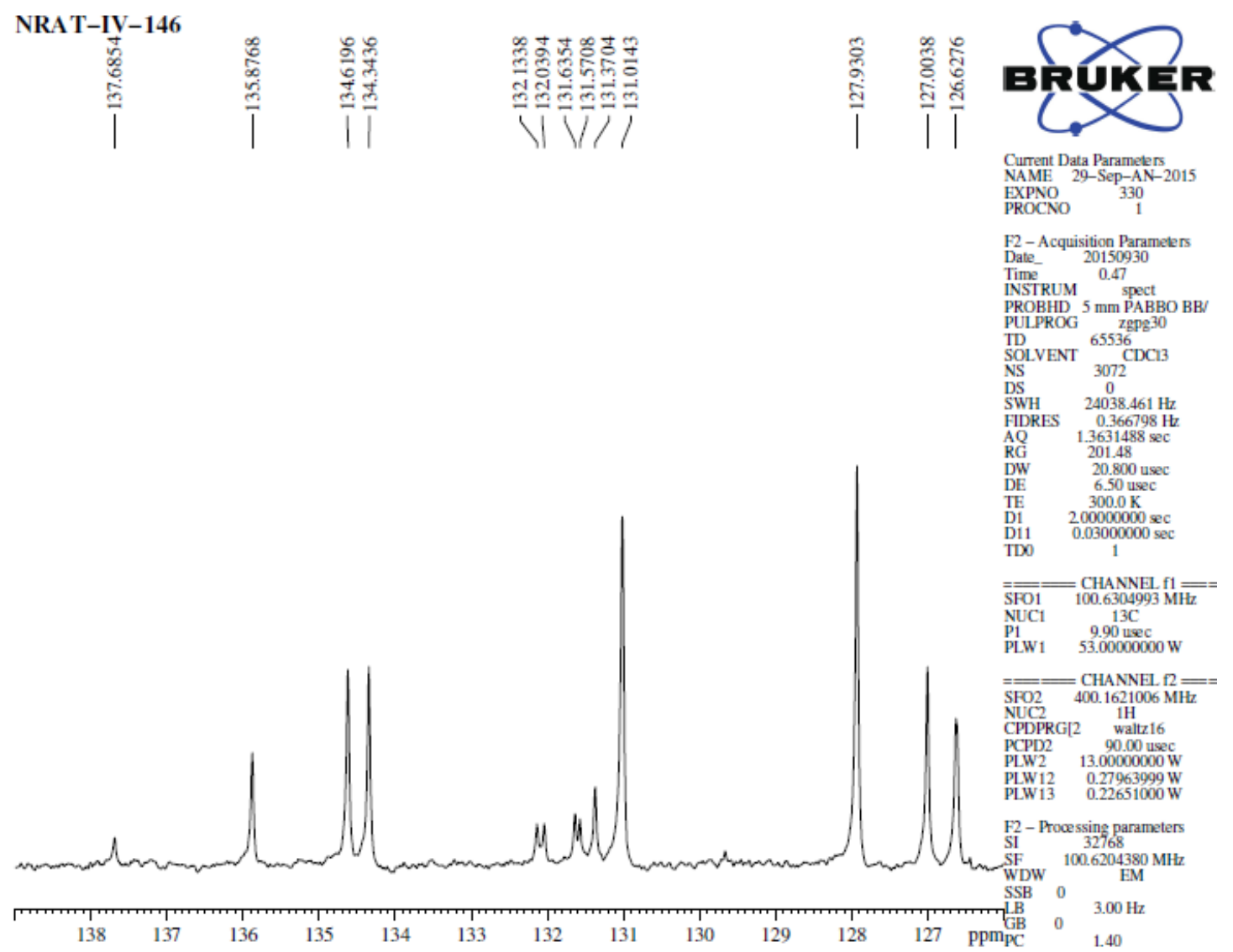

Figure 132: ${ }^{13} \mathrm{C}$ NMR spectrum of 5e (expansion) 
<smiles>COC1=C(c2ccc(Cl)cc2)C(=O)c2ccccc2C1=O</smiles>

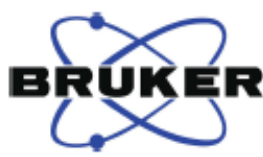

Current Data Parameters

PXPNO

F2 - Acquusition Parameters

$\begin{array}{lc}\text { Date_ } & 2015092 \\ \text { Time } & 14.57\end{array}$

IimsTRUM

PROBHD 5 mm PABBO BE

PULPROG ${ }_{\text {ZgPg } 30}$

${ }_{\text {SOLVENT }}{ }^{65536} \mathrm{CDC1}$

NS $\quad 32$

DS $\quad 40.563 \mathrm{H}$

FIDRES $\quad 0.978127 \mathrm{H}$

AQ 0.5111808 sec

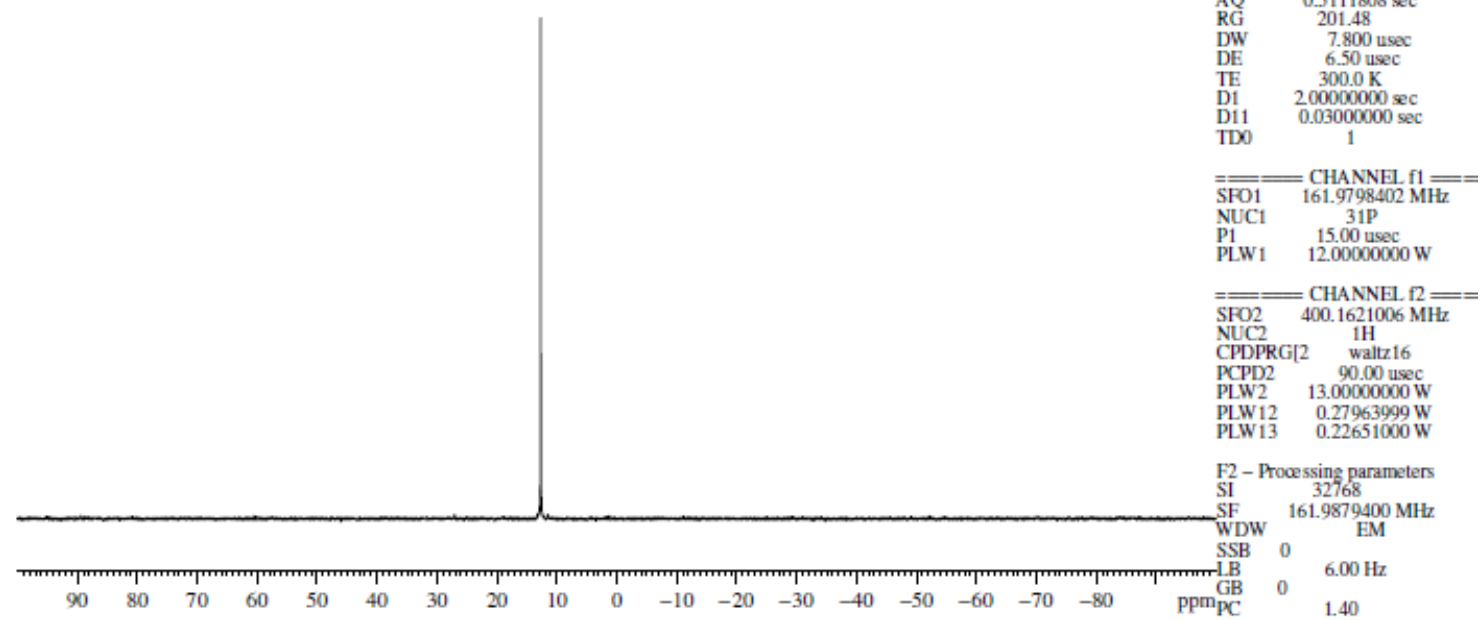

Figure 133: ${ }^{31} \mathrm{P}$ NMR spectrum of $5 \mathrm{e}$

\section{NRAT-IV-159}

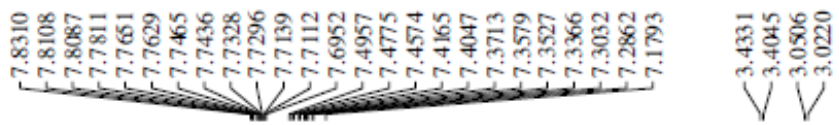<smiles>COC(=O)C1C(=O)c2ccccc2C(=O)C1=C1c2ccccc2-c2ccccc21</smiles>
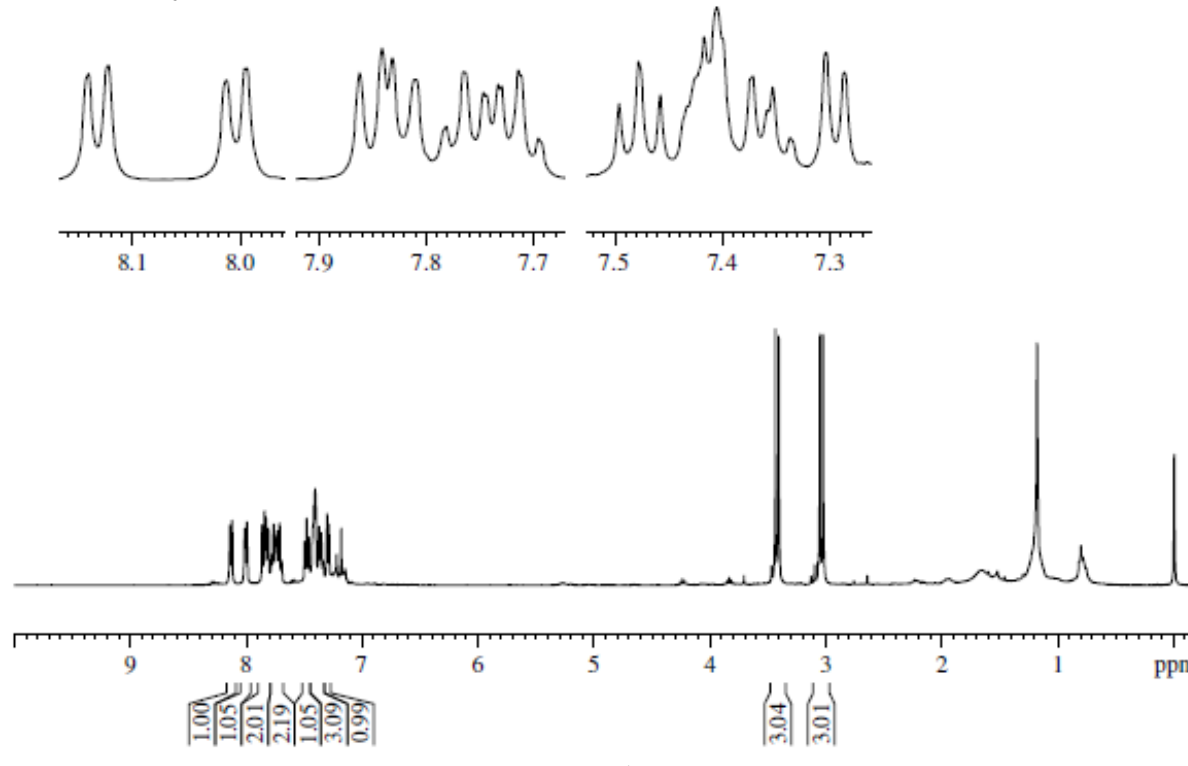

Figure 134: ${ }^{1} \mathrm{H}$ NMR spectrum of $5 f$

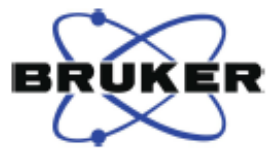

Current Data Parameters
NAME $06-0 c t-F N-2015$ EXPNO $\quad 360$ F2 - Acquisition Parameters
Date
20151006 $\begin{array}{lc}\text { Date_ } & 20151006 \\ \text { Time } & 15.13\end{array}$ INSTRUM
PROBHD $5 \mathrm{~mm}$ PABE
PULPOG TD $65536 \mathrm{~g} 30$ SOLVENT CDCI3 $\begin{array}{ll}\text { NS } & 8 \\ \text { DS } & 0\end{array}$ SWH $\quad 9615.385 \mathrm{~Hz}$ FIDRES $\quad 0.146719 \mathrm{I}$ $\begin{array}{ll}\text { RG } & 80.54 \\ \text { DW } & 52.000\end{array}$ DE 6.50 usec $\begin{array}{lc}\text { TE } & 300.0 \mathrm{~K} \\ \text { D1 } & 1.00000000 \mathrm{sec} \\ \text { TD0 } & 1\end{array}$ $=\overline{\overline{S F O 1}}$
NUC1
CHANNEL $1629712 \mathrm{MHz}$ $\begin{array}{ll}\text { P1 } & 13.20 \text { usec } \\ \text { PLW1 } & 13.00000000 \mathrm{~W}\end{array}$ F2 - Prowssing parameter SI $\quad 65936$ SF $400.1605414 \mathrm{MHz}$ $\mathrm{SSB}_{0} \quad \mathrm{EM}$ $\stackrel{1 B}{\mathrm{G}} \quad 0.30 \mathrm{~Hz}$ $\begin{array}{lll}\mathrm{GB} & 0 & 1.00 \\ \mathrm{PC} & & \end{array}$ 


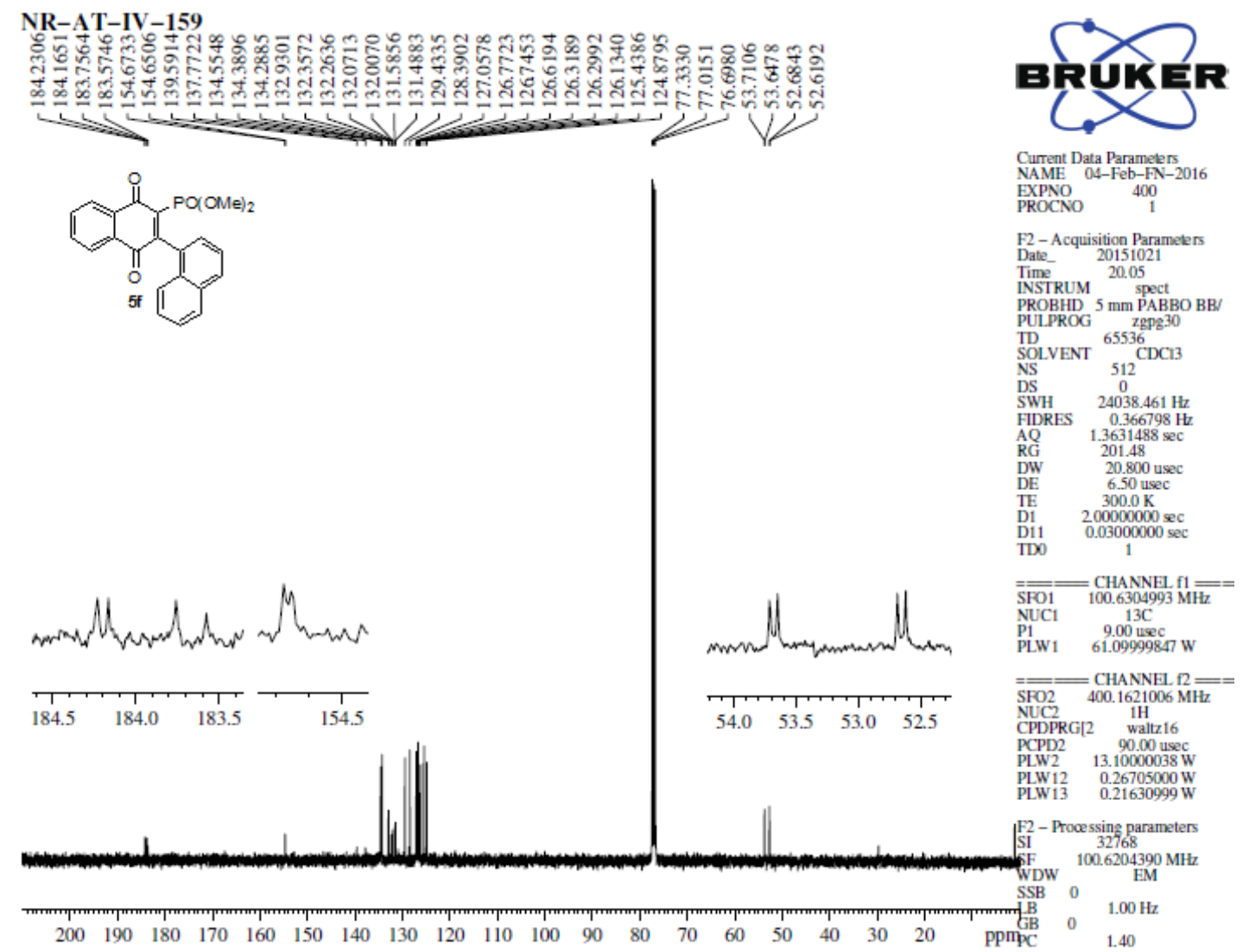

Figure 135: ${ }^{13} \mathrm{C}$ NMR spectrum of $5 f$

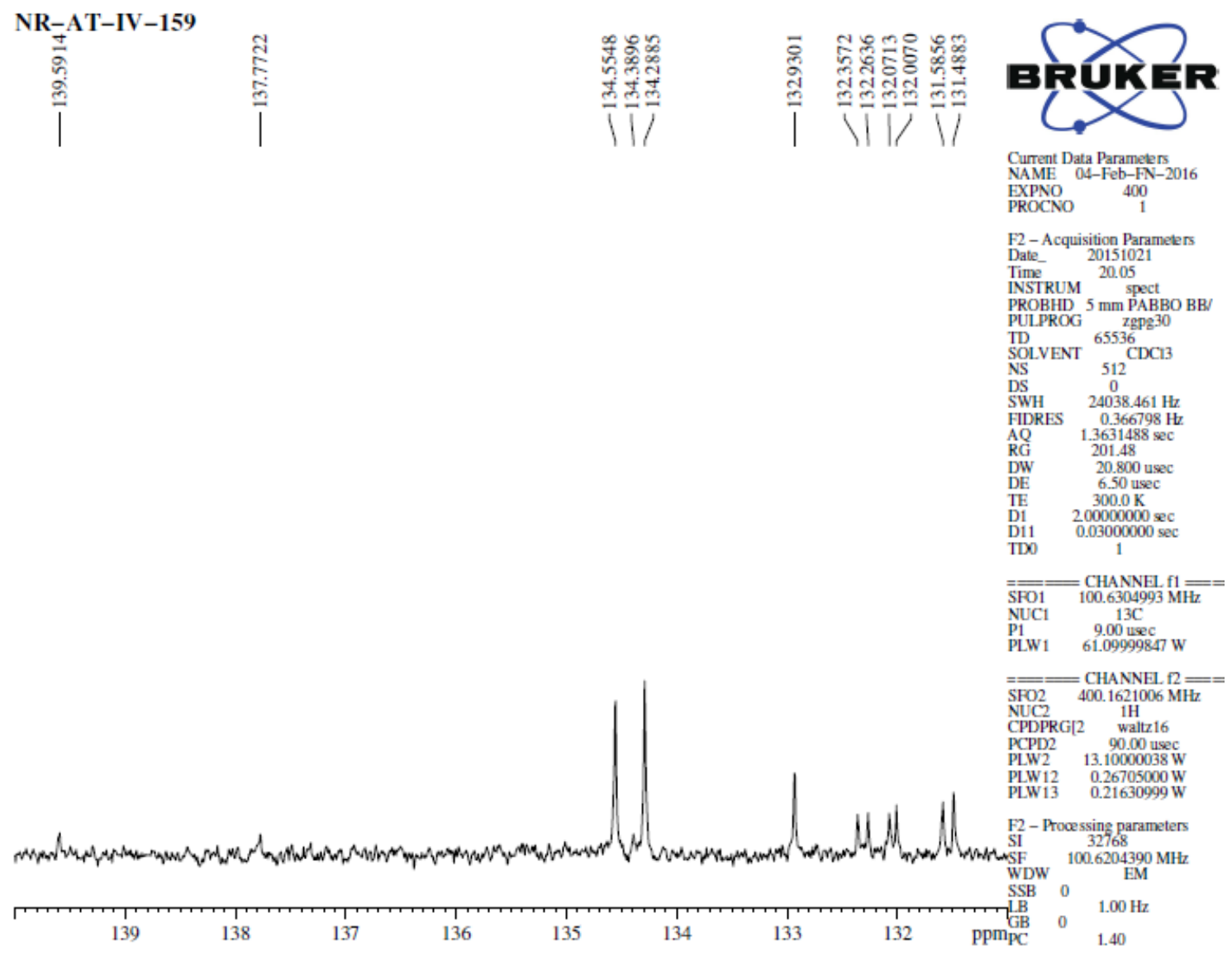

Figure 136: ${ }^{13} \mathrm{C}$ NMR spectrum of $5 f$ (expansion) 


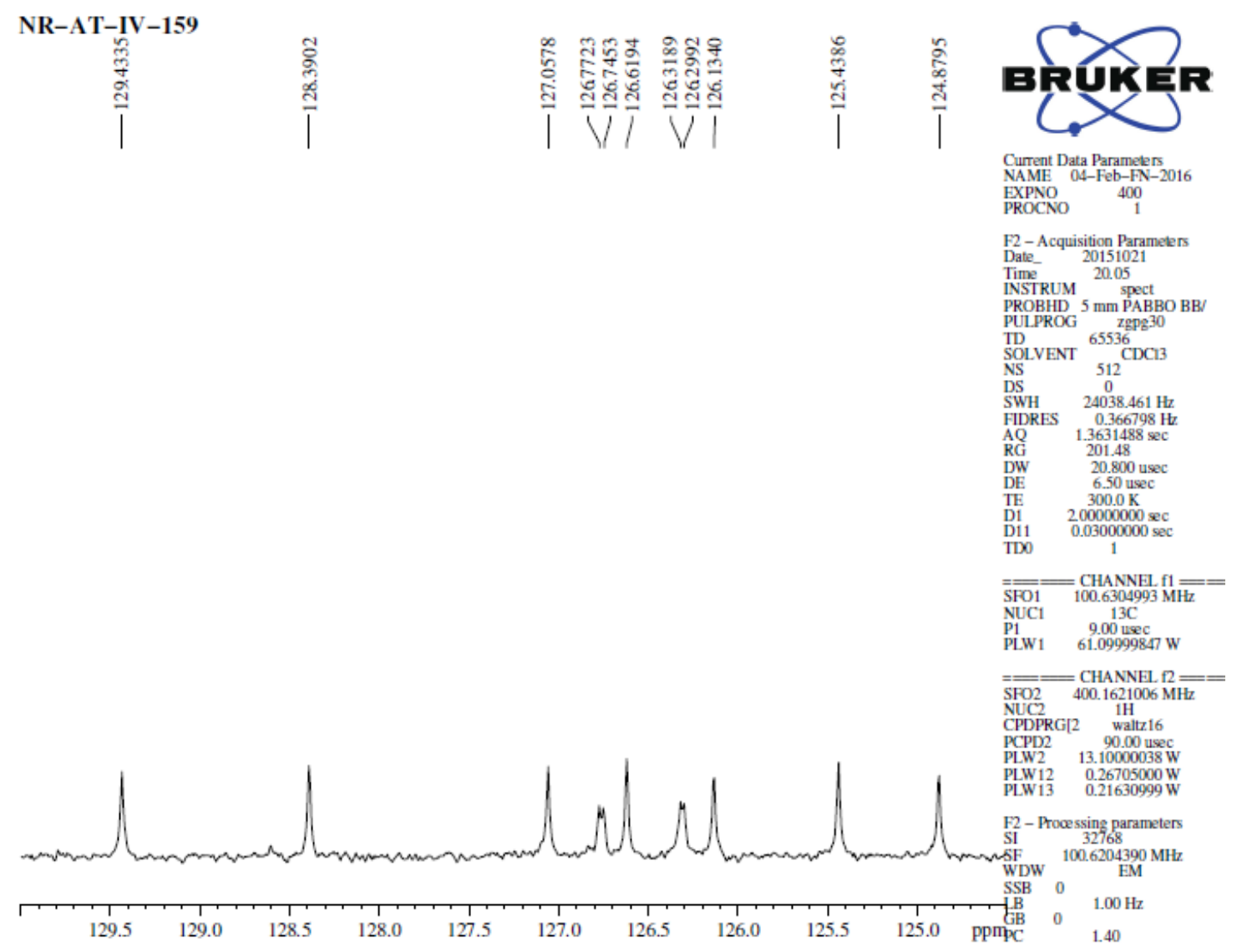

Figure 137: ${ }^{13} \mathrm{C}$ NMR spectrum of $5 f$ (expansion-2)

NR-AT-IV-159<smiles>COC1=C(c2cccc3ccccc23)C(=O)c2ccccc2C1=O</smiles>
Cuncent Data Parameters
NAME 12 -OCA-AN-2015 EXPNO
PROCNO

F2-Acquisibon Paramcten

Date_ 20151013

Time 200

INSTRLM 5 mm PMA PULROG 2 TgPe30 SOLVENT $\quad \mathrm{CDO}^{-}$ $\begin{array}{lcl}\text { NS } & 16 \\ \text { DS } & 4 \\ \text { SWH } & 64102563\end{array}$

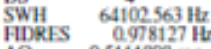
${ }_{A O} 0.5111808$

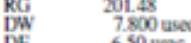

DE $\quad 350$ weec

Di. $20000000 \mathrm{x}$ TD0 1

$\overline{\text { SFO1 }}=\underset{161.9798402}{\text { CHAN }} \mathrm{MHz}=$ NUC1 31P

P1.w1 13.25 weec 19099961 w

$\overline{\overline{\mathrm{SFO} 2}}=$ CHANAH. $\mathrm{CH}=$ $\mathrm{NUC2}_{2}$ ill CPDRGG|2 walu16

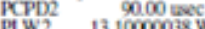
PLW12 03605000 PLW13 0.21630999 w

F2- Processing purameter SF $161.9879400 \mathrm{MHz}$ SSB 0

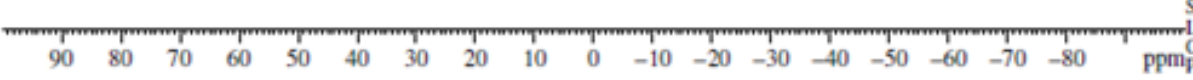

Figure 138: ${ }^{31} \mathrm{P}$ NMR spectrum of $5 f$ 
NRAT-IV-171

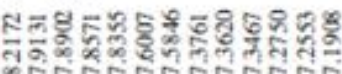

$M V V$ जिए<smiles>COC(=O)C1=C(c2ccccc2Br)C(=O)c2cc(Br)ccc2C1=O</smiles>

59
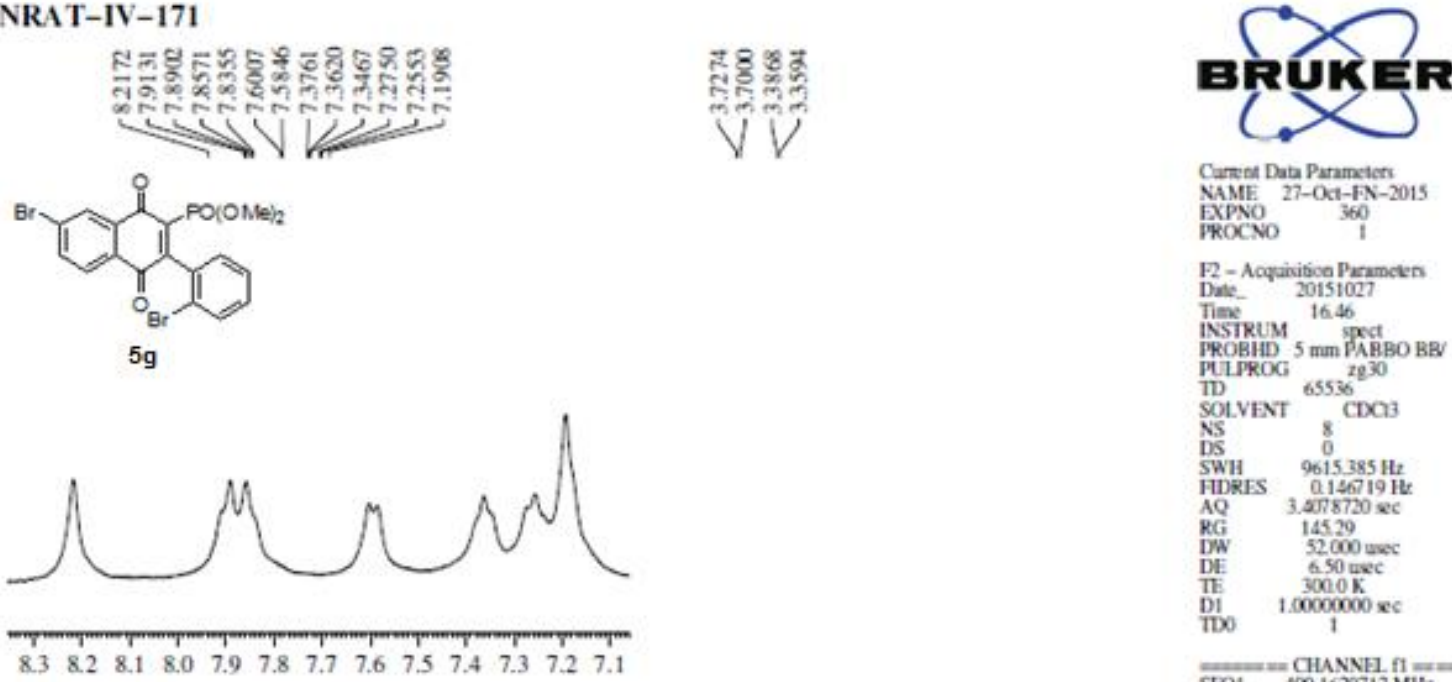

Curnet Data Parameteri

SAME: 27-0K-FN-2015

BROC

P2-Acquakibion Prameten

Time 16.46

INSTKuM sxat

PROBHD $5 \mathrm{~mm}$ PABBO
PUIPROG

II HAROG $65536^{2230}$

SOLVENT CDOS

NS 8 (D)

DS $\quad 0015.385 \mathrm{~Hz}$

FIDRES 0106191

AQ $\quad 3.2578720$

$\begin{array}{ll}\text { KG } & 14529 \\ \text { DW } & 52000 \text { une }\end{array}$

DH $300 \mathrm{~K}$

Di $1.00000000 \times$

$\begin{array}{lllllllllllllll}8.3 & 8.2 & 8.1 & 8.0 & 7.9 & 7.8 & 7.7 & 7.6 & 7.5 & 7.4 & 7.3 & 7.2 & 7.1\end{array}$

SF01 $=$ CHANNE1 $\mathrm{fI}=$ SFO1 $\$ 00.1620712 \mathrm{Min}$

pit 1285

PHW $13,1000003 \mathrm{w}$

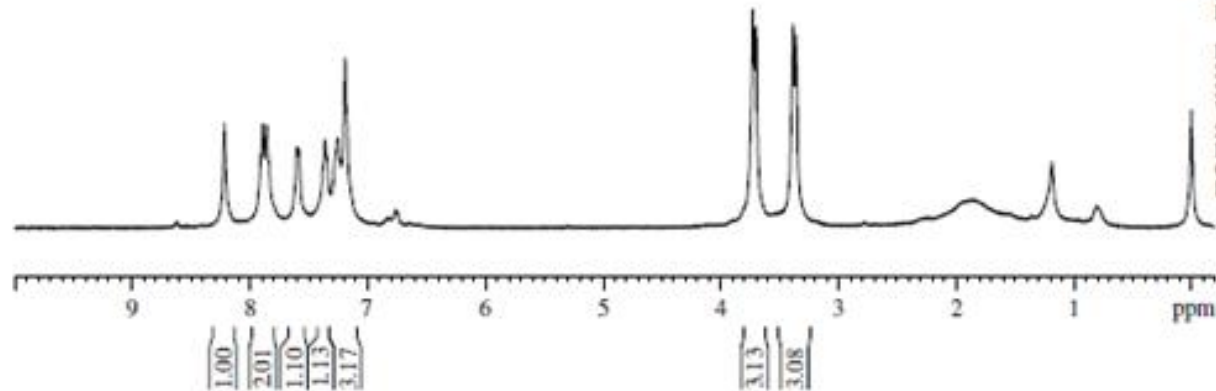

F2-Processing puenenter SF $4001606356 \mathrm{MHL}$ WDW EM

IB $0030 \mathrm{H}$

Ge $0 \quad 1.00$

Figure 139: ${ }^{1} \mathrm{H}$ NMR spectrum of $5 \mathrm{~g}$

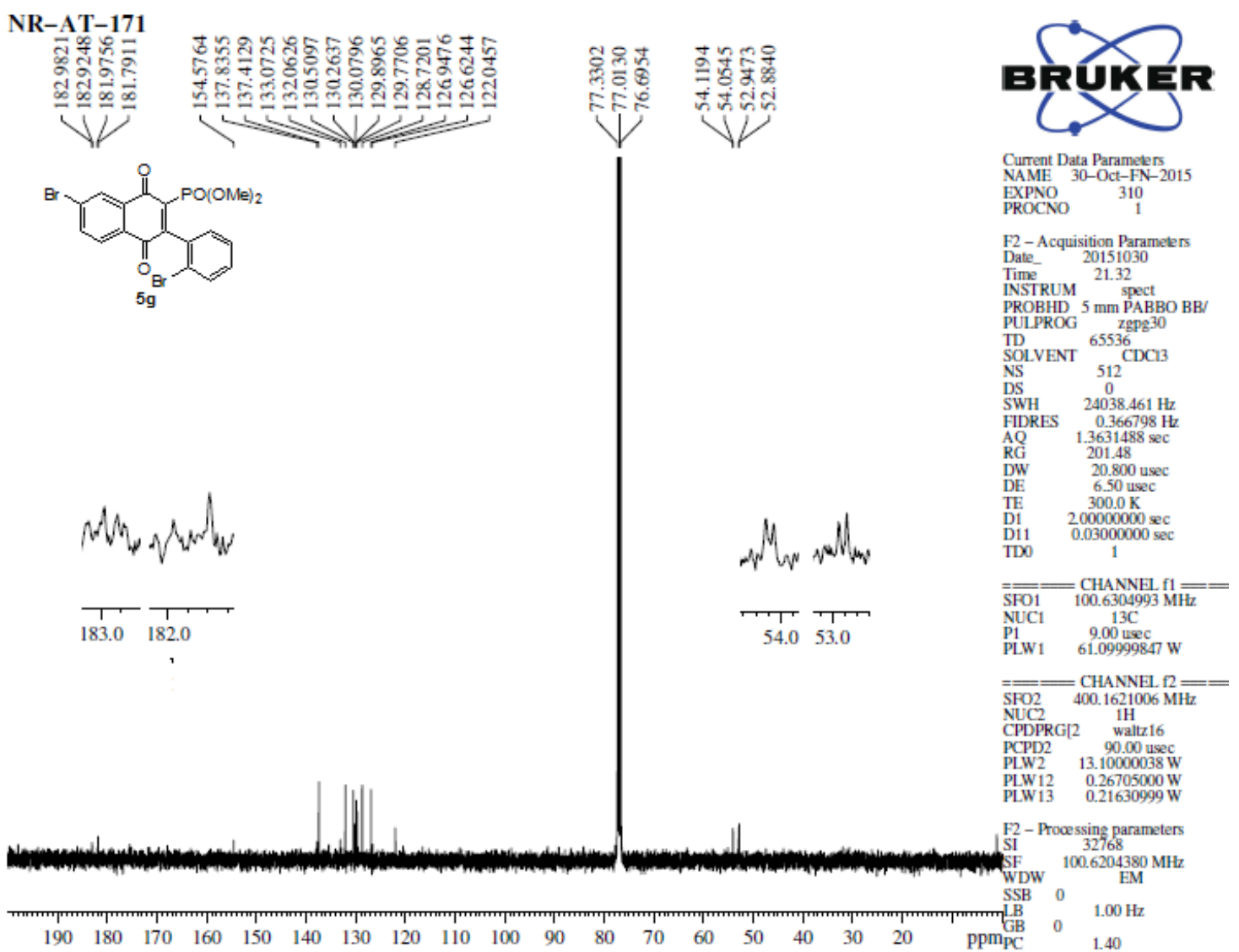

Figure 140: ${ }^{13} \mathrm{C}$ NMR spectrum of $5 \mathrm{~g}$ 


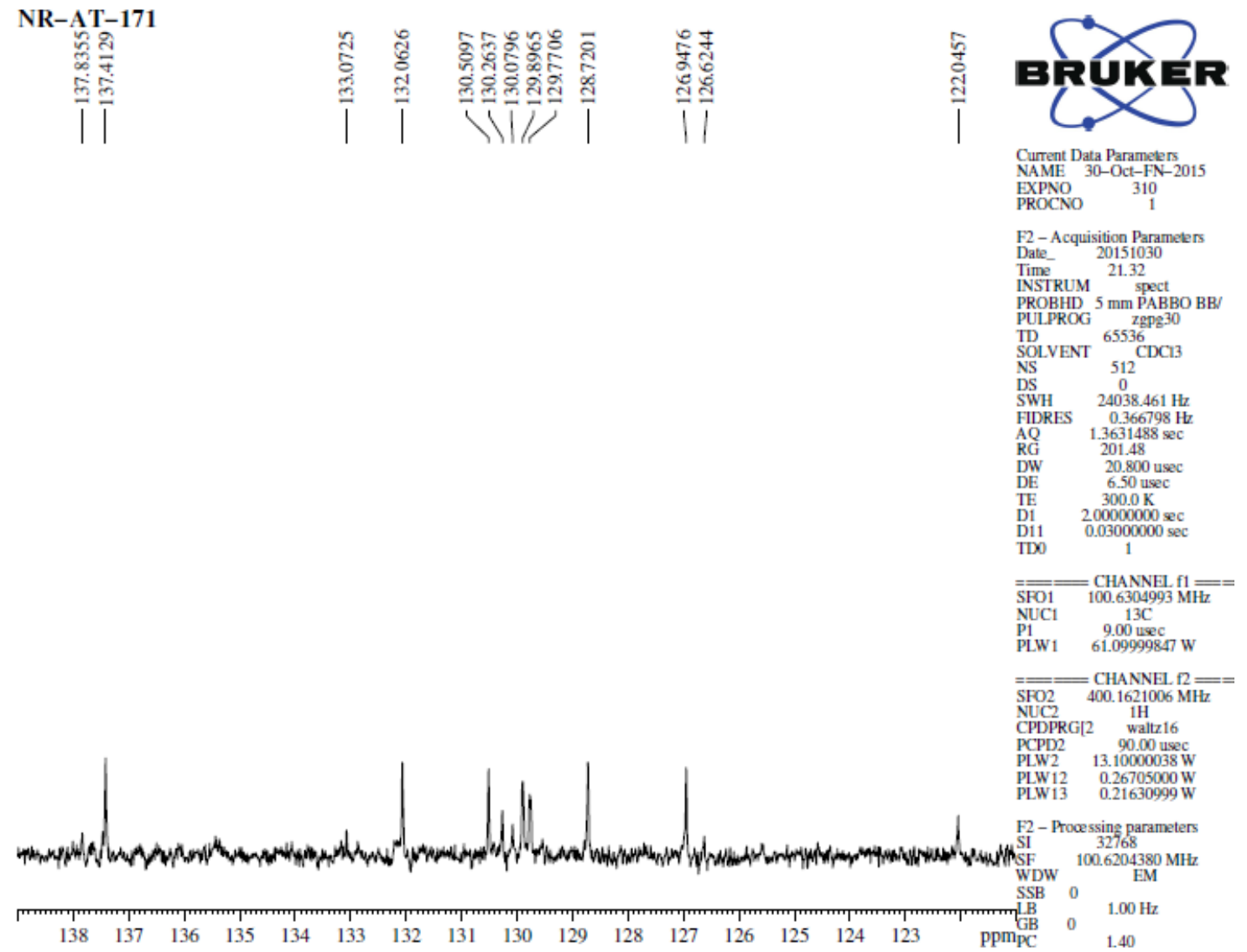

Figure 141: ${ }^{13} \mathrm{C}$ NMR spectrum of $5 \mathrm{~g}$ (expansion)

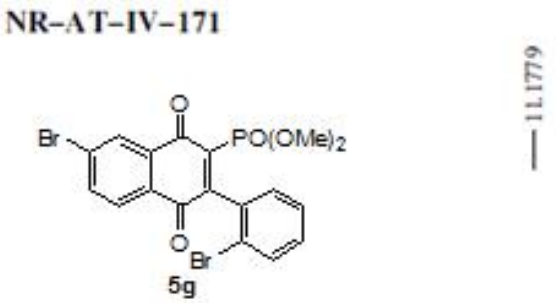

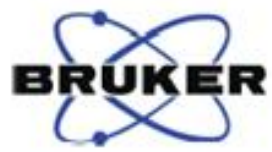

Cursent Dus Parameter NAME 28-OCt-AN-2015 ProcNo

F2-Acquisibion Parametes
Dale 20151029

Time 4.55

SSTKLM stect

PULROG 238230

TD ${ }^{65536}$

SOL.VENT ${ }_{16}^{\text {CDCI }}$

DS

SWH $64102563 \mathrm{H}$

AQ $0.5111805 \mathrm{ec}$

RG 201.45

$\begin{array}{ll}\text { DE } & 650 \mathrm{usec} \\ \text { DI } & 3002 \mathrm{~K}\end{array}$

bil $0.03000000 \mathrm{sec}$

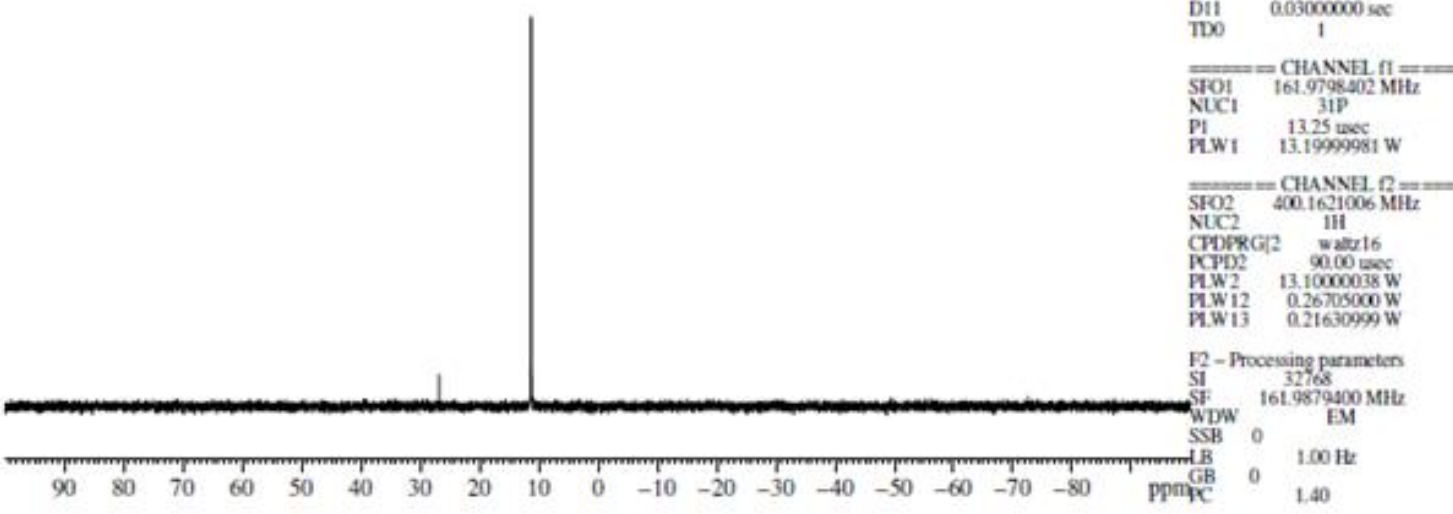

Figure 142: ${ }^{31} \mathrm{P}$ NMR spectrum of $5 \mathrm{~g}$ 


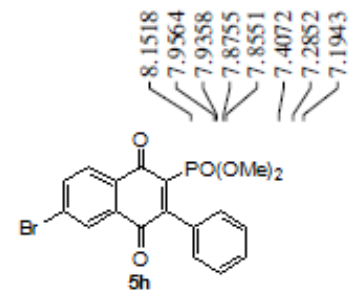

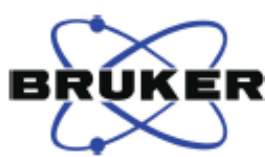

Current Data Parameters $\begin{array}{ll}\text { NAME } & 11-J a n-F N-20 \\ \text { EXPNO } & 380\end{array}$

F2 - Acquisition Parameter

Date_ $\quad 20160112$

Time 18.59

INSTRUM spect

${ }_{\text {PULPROG }}^{\text {PROBHD }} 5 \mathrm{maBBO}$

ID 65536

TDOLVENT ${ }_{\text {CDC13 }}^{65536}$

NS

SWH $\quad 9615.385 \mathrm{~Hz}$

FIDRES $\quad 0.146719 \mathrm{H}$

AQ $\quad 3.4078720$

DW $\quad 52.000$ usec

DE $\quad 6.50$ usec

TE $\quad 300.0 \mathrm{~K}$

D1 $\quad \begin{array}{cc}1.00000000 \mathrm{sec} \\ \text { TD0 }\end{array}$

$=$ CHANNEL $\mathrm{f1}=$

SFO1 $400.1629712 \mathrm{M}$

$\begin{array}{ll}\text { P1 } & 13.20 \text { usec } \\ \text { PLW1 } & 13.00000000 \mathrm{~W}\end{array}$

F2 - Processing parameters

$\begin{array}{ll}\text { SI } & 65536 \\ \text { SF } & 400.1605364 \mathrm{MHz}\end{array}$

WDW $\quad$ EM

$\begin{array}{lll}\text { SSB } & 0 & \\ \text { LB } & & 0.30 \mathrm{~Hz}\end{array}$

$\underset{\mathrm{GB}}{\mathrm{DC}} \quad 0$

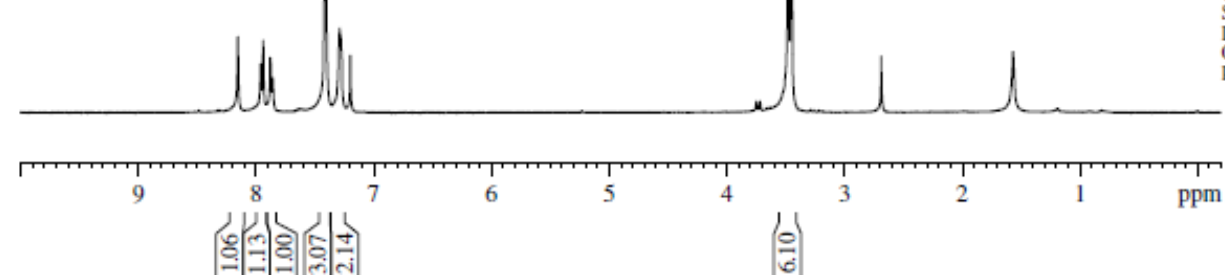

Figure 143: ${ }^{1} \mathrm{H}$ NMR spectrum of $5 \mathrm{~h}$
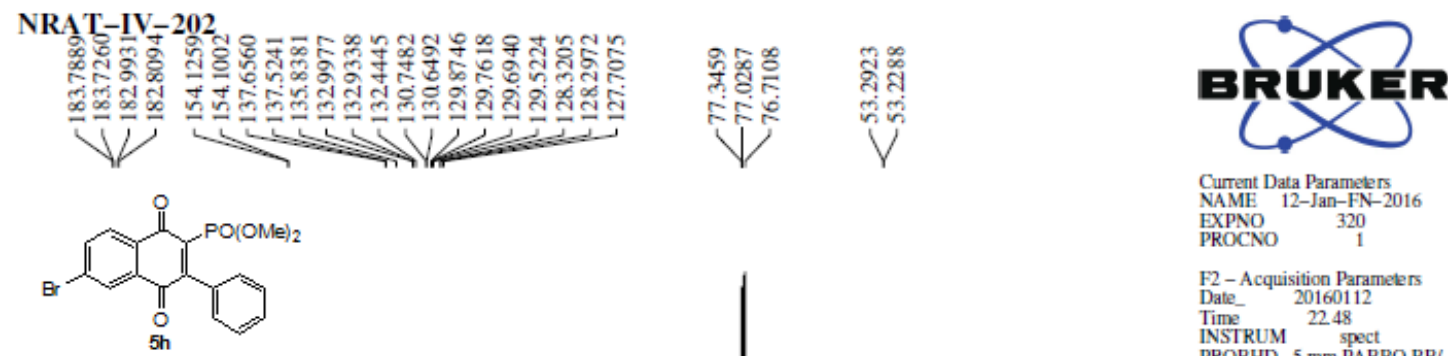

Current Data Parameters

EXPNO 320

F2 - Acquisition Parameter

F2-Acquisition Param
Date_ 20160112

Time 2248

INSTRUM spect

PROBHD 5 mm PABBO

TD.PROG $655366^{\text {ZgP:30 }} 30$

${ }_{\text {SOLVENT }}^{65536}$ CDC13

$\begin{array}{lc}\text { NS } & 512 \\ \text { DS } & 0 \\ \text { SWH } & 24038.461 \mathrm{~Hz}\end{array}$

DRES $\quad 0.366798 \mathrm{H}$

AQ $\quad 1.3631488 \mathrm{sec}$

DW 20.800 usec

$\quad 300.0 \mathrm{~K}$

$\begin{array}{ll}\text { TE } & 300.0 \mathrm{~K} \\ \mathrm{D} 1 & 200000000 \mathrm{sec}\end{array}$
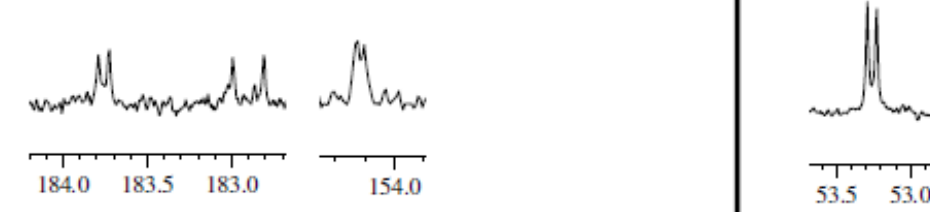

$0.03000000 \mathrm{sec}$

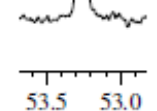

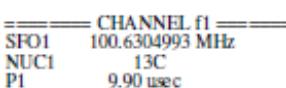

PLW1 $5300000000 \mathrm{~W}$

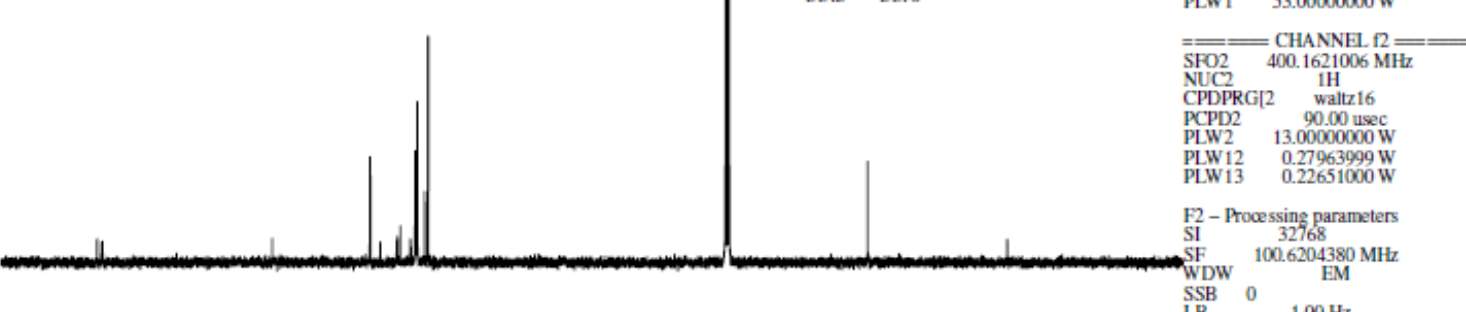

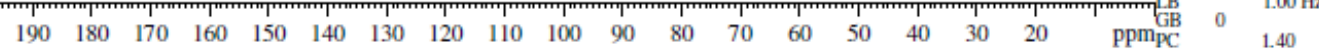

Figure 144: ${ }^{13} \mathrm{C}$ NMR spectrum of $5 \mathrm{~h}$ 


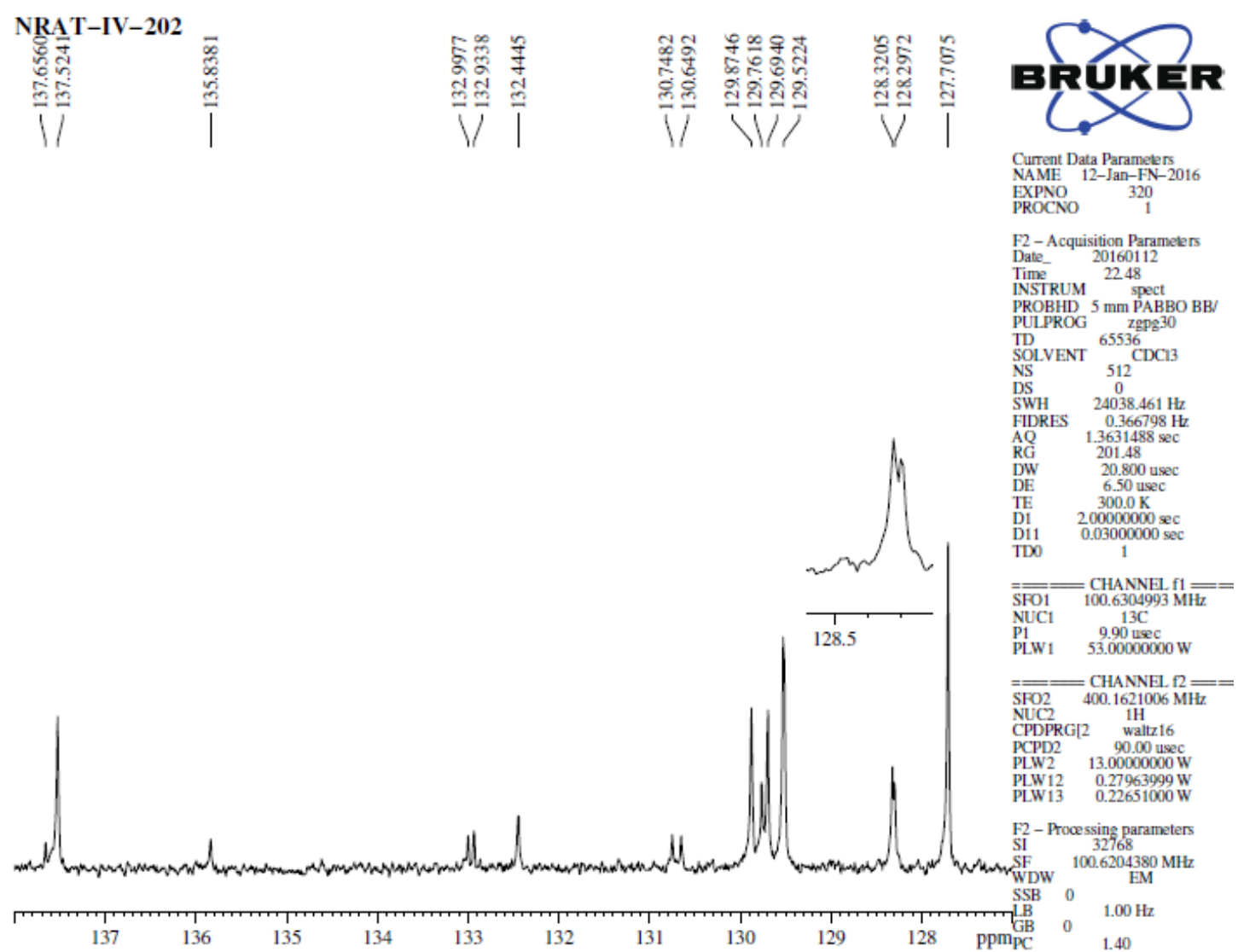

Figure 145: ${ }^{13} \mathrm{C}$ NMR spectrum of $5 \mathrm{~h}$ (expansion)

NRAT-IV-202<smiles>[B]c1ccc2c(c1)C(=O)C(=O)C(=C1C(=O)C(=O)c3ccccc31)C2=O</smiles>

Current Data Paramelers $\begin{array}{lll}\text { EXPNO } & 390 \\ \text { PROCNO } & 1\end{array}$

F2-Acquisition Parameters

Date_
Time 19.06

PROBRD 5 spect

PULPROG Zape 30

TD ${ }_{\text {SOLVENT }}{ }^{65536} \mathrm{CDC13}$

SOLVENT ${ }_{64}^{\text {CDC }}$

DS

SWH $\quad 404102563 \mathrm{~Hz}$

FIDRES $\quad 0.978127 \mathrm{H}$

AQ $\quad 0.5111808 \mathrm{sec}$

RG 201.48

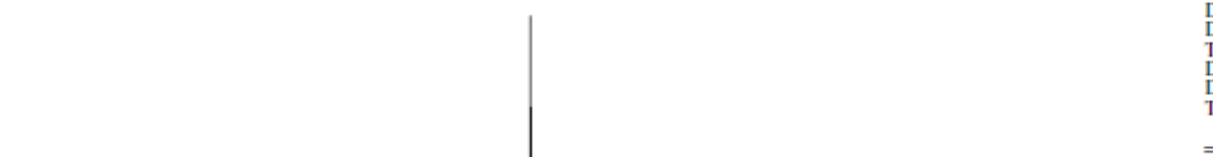

7.800 usec

6.50 usec 200000000 $0.03000000 \mathrm{sec}$

$=$ CHANNE $\mathrm{f} 1=$

NUC1 161.9798402

$\begin{array}{ll}\text { NUC1 } & 31 \mathrm{P} \\ \mathrm{P} 1 & 1500\end{array}$

$\begin{array}{ll}\text { P1 } & 15.00 \text { usec } \\ \text { PLW1 } & 12.00000000 \mathrm{~W}\end{array}$

$=$ CHANNEL $\mathrm{f} 2=$

NUC2 $1 \mathrm{H}$

Taltz16

$\begin{array}{lr}\text { PCPD2 } & 90.00 \text { usec } \\ \text { PLW2 } & 13.00000000 \mathrm{~W}\end{array}$

$\begin{array}{ll}\text { PLW12 } & 0.27963999 \mathrm{~W} \\ \text { PLW13 } & 0.22651000 \mathrm{~W}\end{array}$

F2 - Processing parameters SF $\quad \begin{array}{ll}32768 \\ \text { WFW }\end{array}$

Figure 146: ${ }^{31} \mathrm{P}$ NMR spectrum of $5 \mathrm{~h}$ 